\title{
Visible Light-Promoted Aliphatic C-H Arylation Using Selectfluor as a Hydrogen Atom Transfer Reagent
}

Hong Zhao, and Jian Jin*

\section{Table of Contents}

I. General Information

II. Mechanistic Studies

III. Reaction Setup

IV. Experimental Procedures and Product Characterization

V. NMR Spectra 


\section{General Information}

Commercial reagents, lepidine, $N$-Chloromethyl- $N$ '-fluorotriethylenediammonium bis(tetrafluoroborate) (Selectfluor), cyclohexane, hydrochloric acid ( $\mathrm{HCl})(36 \%-38 \%)$, trifluoroacetic acid (TFA), acetone and acetonitrile were purchased from J\&K, TCI, Acros, Sigma-Aldrich and Fisher Chemical, and used directly without purification. All heteroarenes, alkanes, ketones, esters, ethers and other reagents were utilized directly from commercial suppliers. Organic solutions were concentrated under reduced pressure on a Büchi rotary evaporator with a recirculating cooling system. Chromatographic purification of products was accomplished by flash chromatography on silica gel (Santai, 230-400 mesh). Thin layer chromatography (TLC) was performed on Huanghai 0.4-0.5 $\mathrm{mm}$ silica gel plates. Visualization of the developed chromatogram was performed by fluorescence quenching. ${ }^{1} \mathrm{H}$ and ${ }^{13} \mathrm{C}$ NMR spectra were recorded on a Bruker UltraShield Plus $400 \mathrm{MHz}(101 \mathrm{MHz})$ instrument, and are internally referenced to residual protio solvent signals (note: $\mathrm{CDCl}_{3}$ referenced at 7.26 and $77.0 \mathrm{ppm}$ respectively). Data for ${ }^{1} \mathrm{H}$ NMR are reported as follows: chemical shift $(\delta \mathrm{ppm})$, multiplicity $(\mathrm{s}=$ singlet, $\mathrm{d}=$ doublet, $\mathrm{t}=$ triplet, $\mathrm{q}=$ quartet, $\mathrm{m}=$ multiplet, $\mathrm{dd}=$ doublet of doublets, $\mathrm{dt}=$ doublet of triplets, $\mathrm{br}=$ broad), coupling constant $(\mathrm{Hz})$ and integration. Data for ${ }^{13} \mathrm{C}$ NMR are reported in terms of chemical shift and no special nomenclature is used for equivalent carbons. IR spectra were recorded on a Perkin Elmer Spectrum 100 FTIR spectrometer and are reported in wavenumbers $\left(\mathrm{cm}^{-1}\right)$. High resolution mass spectra were obtained at National Center for Organic Mass Spectrometry in Shanghai Institute of Organic Chemistry, Chinese Academy of Sciences on a Thermo Fisher Scientific LTQ FTICRMS instrument with electrospray ionization method. 


\section{Mechanistic Studies}<smiles>O=[N+]([O-])c1ccnc2ccccc12</smiles>

$0.5 \mathrm{mmol} 10$<smiles>C1CCCCC1</smiles>

20 equiv. 3

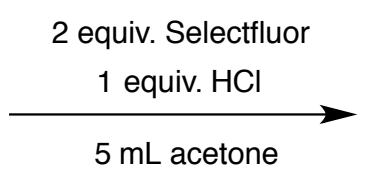

$427 \mathrm{~nm}$ LED, fan, rt, $16 \mathrm{~h}$<smiles>O=[N+]([O-])c1cc(C2CCCCC2)nc2ccccc12</smiles>

11

\begin{tabular}{cccc} 
Entry & Radical Scavenger & SM 10 & Product 11 \\
\hline 1 & TEMPO, 0.5 equiv., $[0.05 \mathrm{M}]$ & $53 \%$ & $26 \%$ \\
2 & TEMPO, 1.0 equiv., $[0.10 \mathrm{M}]$ & $77 \%$ & $5 \%$ \\
3 & TEMPO, 1.5 equiv., $[0.15 \mathrm{M}]$ & $82 \%$ & $0 \%$ \\
4 & air residue in acetone and headspace, $\mathrm{O}_{2}<[0.011 \mathrm{M}]$ & $0 \%$ & $90 \%$ \\
5 & $\mathrm{O}_{2}$ sparging, $\mathrm{O}_{2}[0.011 \mathrm{M}]$ & $9 \%$ & $81 \%$ \\
6 & $\mathrm{O}_{2}$ sparging, $\mathrm{O}_{2}$ ballon, $\mathrm{O}_{2}>[0.011 \mathrm{M}]$ & $40 \%$ & $46 \%$ \\
7 & 1,1 -diphenylethylene, 3.0 equiv., $[0.30 \mathrm{M}]$ & $66 \%$ & $26 \%$ \\
8 & 1,1 -diphenylethylene, 4.0 equiv., $[0.40 \mathrm{M}]$ & $91 \%$ & $0 \%$
\end{tabular}

Yield determined by ${ }^{1} \mathrm{H}$ NMR using 1,3-benzodioxole as the internal standard after work-up.

To an $8 \mathrm{~mL}$ vial equipped with a Teflon septum and a magnetic stir bar was charged lepidine (71.6 $\mathrm{mg}, 0.50 \mathrm{mmol}, 1.0$ equiv.), Selectfluor (373.0 mg, $1.0 \mathrm{mmol}, 2.0$ equiv.), indicated amount of radical scavenger, $5.0 \mathrm{~mL}$ of acetone. The reaction mixture was degassed by sparging with nitrogen for 10 min with an outlet needle, and added with hydrochloric acid ( $43.0 \mu \mathrm{l}, 0.50 \mathrm{mmol}, 1.0$ equiv.), cyclohexane $(1.1 \mathrm{~mL}, 10.0 \mathrm{mmol}$, 20.0 equiv.), then irradiated with Kessil $40 \mathrm{~W} 427 \mathrm{~nm}$ LEDs (approximately $8 \mathrm{~cm}$ away from the light source) under two mini fans at room temperature. After 16 hours, the reaction mixture was diluted with $1.0 \mathrm{M} \mathrm{NaOH}$ aqueous solution $(20 \mathrm{~mL})$, and extracted by $\mathrm{CH}_{2} \mathrm{Cl}_{2}(3 \times 20 \mathrm{~mL})$, dried over $\mathrm{Na}_{2} \mathrm{SO}_{4}$, and concentrated in vacuo. Yield determined by ${ }^{1} \mathrm{H}$ NMR using 1,3 -benzodioxole as the internal standard.

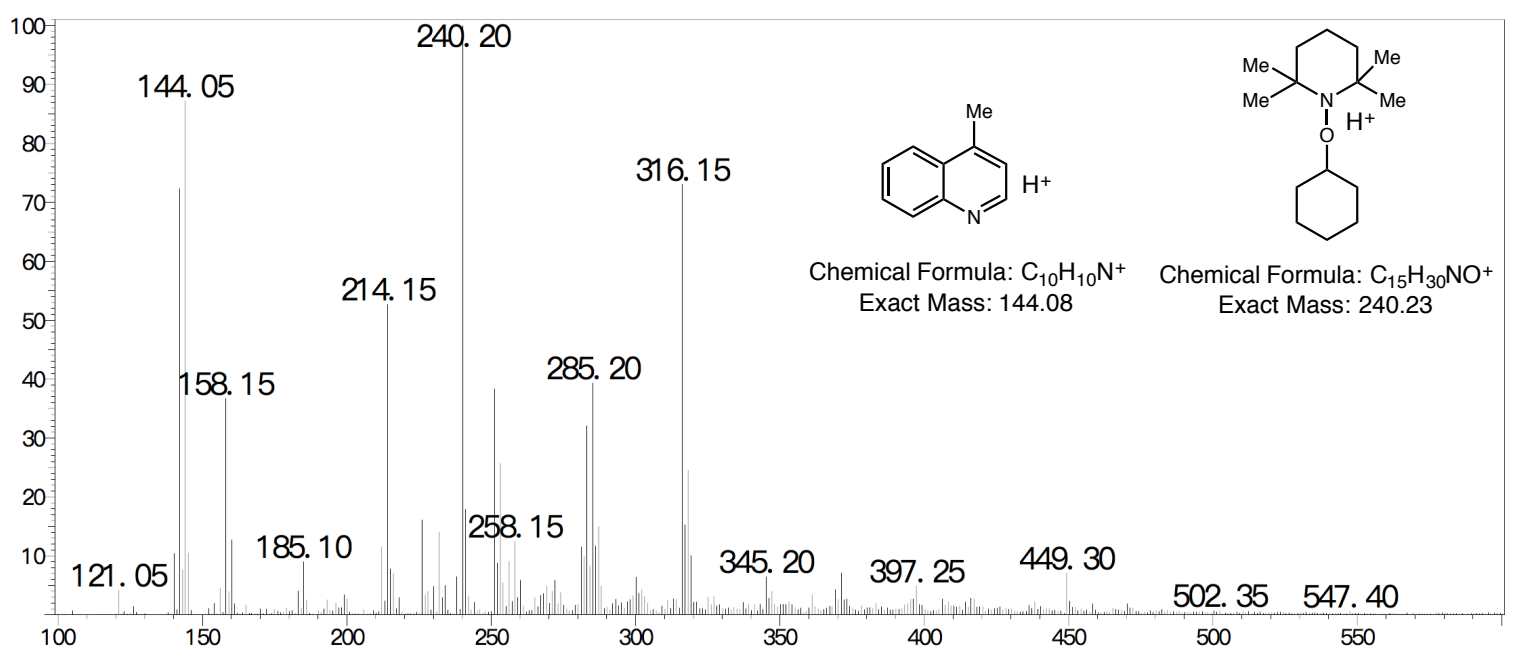

Figure S1. Radical quenching experiments. 
<smiles>Cc1ccnc2ccccc12</smiles>

$0.5 \mathrm{mmol} 10$<smiles>C1CCCCC1</smiles>

20 equiv. 3

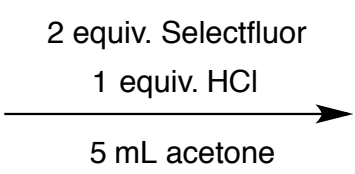

$427 \mathrm{~nm}$ LED, fan, rt<smiles>Cc1cc(C2CCCCC2)nc2ccccc12</smiles>

11

\begin{tabular}{ccc} 
Entry & Light On and Off Conditions & Product \\
\hline 1 & ON 2 h & $32 \%$ \\
2 & ON 2 h, OFF 2 h & $31 \%$ \\
3 & ON 2 h, OFF 2 h, ON 2 h & $50 \%$ \\
4 & ON 2 h, OFF 2 h, ON 2 h, OFF, 2 h & $49 \%$
\end{tabular}

Yield determined by ${ }^{1} \mathrm{H}$ NMR using 1,3-benzodioxole as the internal standard after work-up.

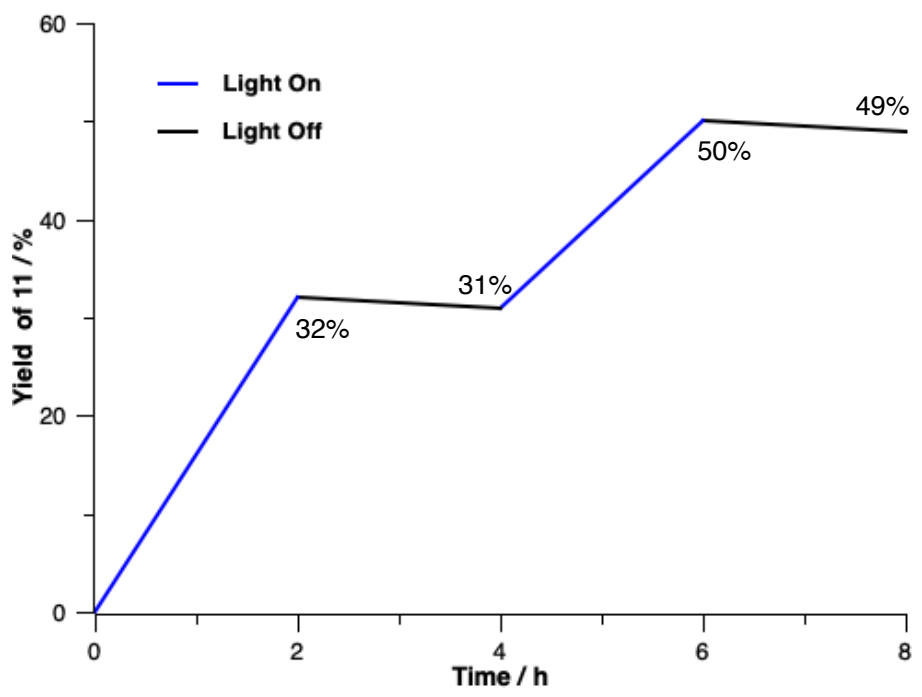

Figure S2. Light on and off experiments.

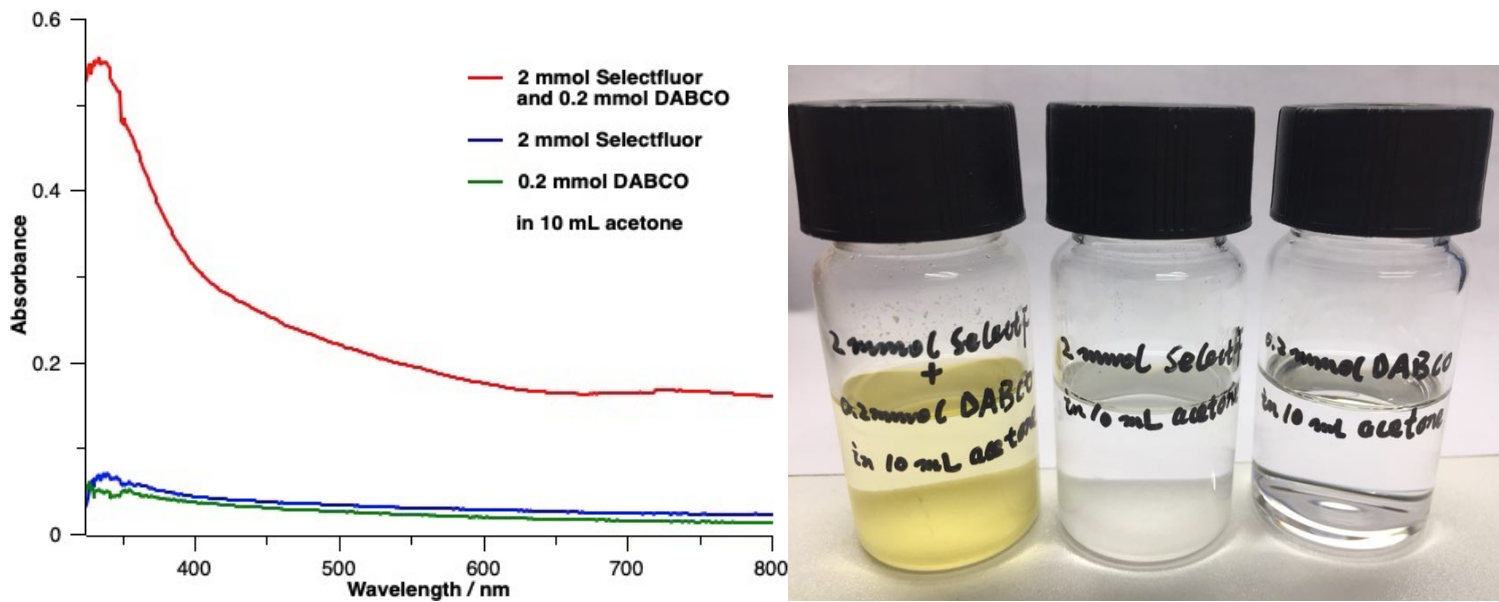

Figure S3. UV-Vis Spectra of Selectfluor and DABCO. 


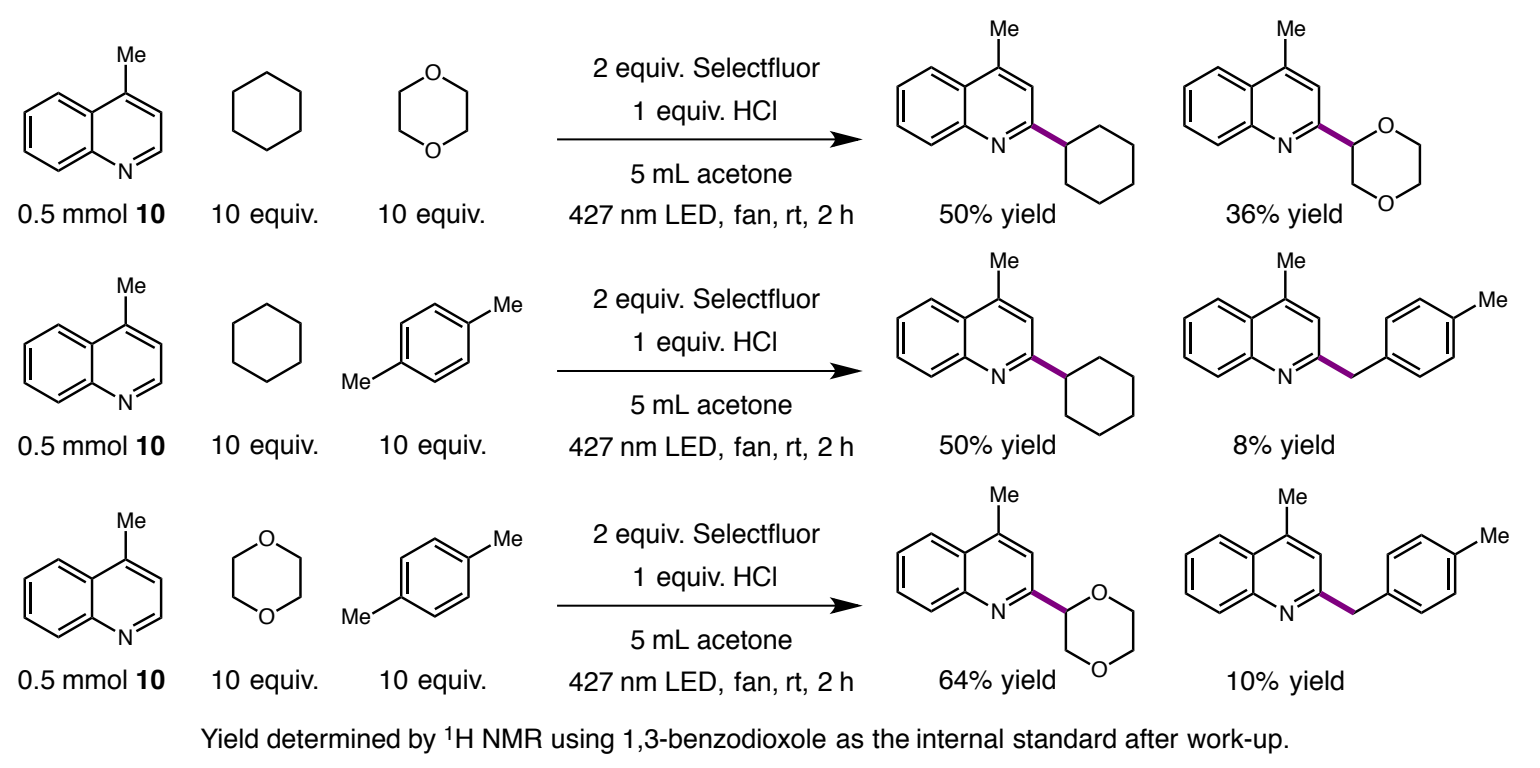

Figure S4. Intermolecular competition experiments.<smiles>Nc1ccnc2ccccc12</smiles>

$0.5 \mathrm{mmol} 10$<smiles>O=[N+]([O-])c1ccnc2ccccc12</smiles>

$0.5 \mathrm{mmol} 10$

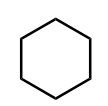

20 equiv. 3

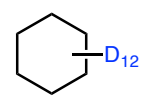

20 equiv.

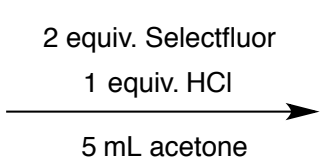

$427 \mathrm{~nm}$ LED, fan, rt, $2 \mathrm{~h}$

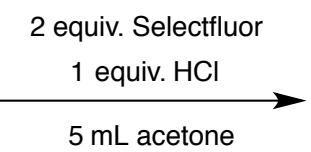

$427 \mathrm{~nm}$ LED, fan, rt, $2 \mathrm{~h}$<smiles>Cc1cc(C2CCCCC2)nc2ccccc12</smiles>

$23 \%$ yield<smiles>Cc1cc(C2CCCCC2)nc2ccccc12</smiles>

$20 \%$ yield<smiles>O=[N+]([O-])c1ccnc2ccccc12</smiles>

$0.5 \mathrm{mmol} 10$<smiles>C1CCCCC1</smiles>

10 equiv. 3<smiles>O=C1CCCCC1</smiles>

10 equiv.

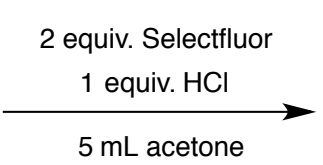

$427 \mathrm{~nm}$ LED, fan, rt, $2 \mathrm{~h}$<smiles>O=[N+]([O-])c1cc(C2CCCCC2)nc2ccccc12</smiles>

$14 \%$ yield<smiles>Cc1cc(C2CCCCC2)nc2ccccc12</smiles>

$9 \%$ yield

Yield determined by ${ }^{1} \mathrm{H}$ NMR using 1,3-benzodioxole as the internal standard after work-up.

\section{Figure S5. KIE experiments.}




\begin{tabular}{|c|c|c|c|c|}
\hline $0.5 \mathrm{mmol} 10$ & 20 equiv. 3 & $\begin{array}{c}2 \text { equiv. Selectfluor } \\
\text { acid } \\
5 \mathrm{~mL} \text { solvent } \\
40 \mathrm{~W} \text { LED, fan, rt, } 16 \mathrm{~h}\end{array}$ & & 11 \\
\hline Entry & Solvent & Acid & Wavelength & $\%$ Yield[a] \\
\hline 1 & acetone & none & $427 \mathrm{~nm}$ & 14 \\
\hline 2 & acetone & 1 equiv. TFA & $427 \mathrm{~nm}$ & 33 \\
\hline 3 & acetone & 1 equiv. $\mathrm{HCl}$ & $427 \mathrm{~nm}$ & 91 \\
\hline 4 & acetonitrile & 2 equiv. TFA & $427 \mathrm{~nm}$ & 92 \\
\hline 5 & acetonitrile & 2 equiv. $\mathrm{HCl}$ & $427 \mathrm{~nm}$ & 84 \\
\hline 6 & acetone & 1 equiv. $\mathrm{HCl}$ & $440 \mathrm{~nm}$ & 70 \\
\hline 7 & acetone & 1 equiv. $\mathrm{HCl}$ & $456 \mathrm{~nm}$ & 27 \\
\hline 8 & acetone & 1 equiv. $\mathrm{HCl}$ & $467 \mathrm{~nm}$ & 39 \\
\hline 9 & acetone & 1 equiv. $\mathrm{HCl}$ & dark & 0 \\
\hline 10 & acetone & 1 equiv. $\mathrm{HCl}$ & dark, $50^{\circ} \mathrm{C}$ & 0 \\
\hline $11^{[b]}$ & acetone & 1 equiv. $\mathrm{HCl}$ & $427 \mathrm{~nm}$ & 90 \\
\hline $12^{[c]}$ & acetone & 1 equiv. $\mathrm{HCl}$ & $427 \mathrm{~nm}$ & 78 \\
\hline
\end{tabular}

[a] Yield determined by ${ }^{1} \mathrm{H}$ NMR using 1,3-benzodioxole as the internal standard after work-up following the general procedure. [b] Reaction performed without the nitrogen purging. [c] Reaction performed with 1 equivalent Selectfluor. TFA = trifluoroacetic acid. $\mathrm{HCl}=$ hydrochloric acid. LED = light-emitting diode.

Figure S6. Control experiments. 


\section{Reaction Setup}

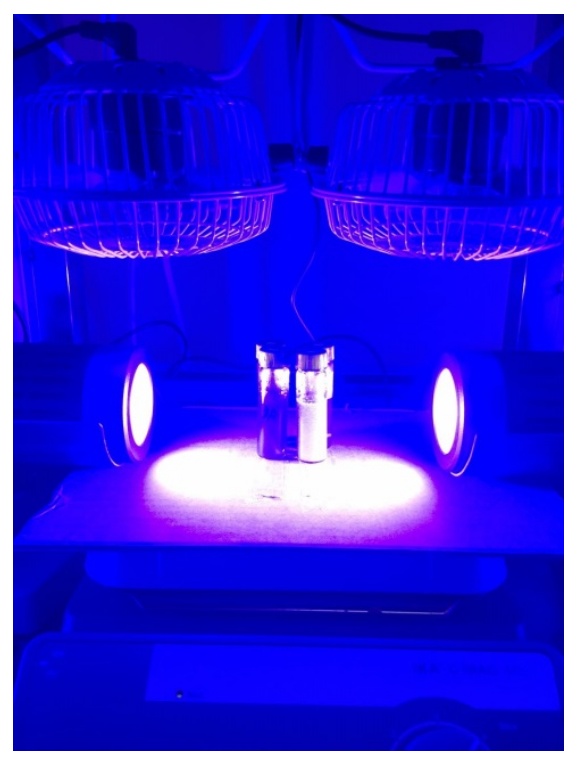

Figure S7. Reaction set-up with a magnetic stirrer, two Kessil 40 W 427 nm LED lamps, two mini fans and four reaction vials. 


\section{Experimental Procedures and Product Characterization}

General Procedure (Conditions A): To an $8 \mathrm{~mL}$ vial equipped with a Teflon septum and a magnetic stir bar was charged heteroarene $(0.50 \mathrm{mmol}, 1.0$ equiv. $)$, Selectfluor (373.0 mg, $1.0 \mathrm{mmol}, 2.0$ equiv.), $5.0 \mathrm{~mL}$ of acetone. The reaction mixture was degassed by sparging with nitrogen for 10 min with an outlet needle, and added with hydrochloric acid (43.0 $\mu \mathrm{l}, 0.50 \mathrm{mmol}, 1.0$ equiv.), alkane ( $10.0 \mathrm{mmol}, 20.0$ equiv.), then irradiated with Kessil $40 \mathrm{~W} 427 \mathrm{~nm}$ LEDs (approximately $8 \mathrm{~cm}$ away from the light source) under two mini fans at room temperature. Upon reaction completion as judged by TLC and LCMS (about 16 hours), the reaction mixture was diluted with $1.0 \mathrm{M} \mathrm{NaOH}$ aqueous solution $(20 \mathrm{~mL})$, and extracted by $\mathrm{CH}_{2} \mathrm{Cl}_{2}(3 \times 20 \mathrm{~mL})$, dried over $\mathrm{Na}_{2} \mathrm{SO}_{4}$, and concentrated in vacuo. Purification of the crude product by flash chromatography on silica gel using the indicated solvent system afforded the desired product.

General Procedure (Conditions B): To an $8 \mathrm{~mL}$ vial equipped with a Teflon septum and a magnetic stir bar was charged heteroarene $(0.50 \mathrm{mmol}, 1.0$ equiv. $)$, Selectfluor (373.0 mg, $1.0 \mathrm{mmol}, 2.0$ equiv.), $5.0 \mathrm{~mL}$ of acetonitrile. The reaction mixture was degassed by sparging with nitrogen for 10 min with an outlet needle, and added with trifluoroacetic acid ( $72.3 \mu 1,1.0 \mathrm{mmol}, 2.0$ equiv.), alkane (10.0 mmol, 20.0 equiv.), then irradiated with Kessil $40 \mathrm{~W} 427 \mathrm{~nm}$ LEDs (approximately $8 \mathrm{~cm}$ away from the light source) under two mini fans at room temperature. Upon reaction completion as judged by TLC and LCMS (about 16 hours), the reaction mixture was diluted with $1.0 \mathrm{M} \mathrm{NaOH}$ aqueous solution $(20 \mathrm{~mL})$, and extracted by $\mathrm{CH}_{2} \mathrm{Cl}_{2}(3 \times 20 \mathrm{~mL})$, dried over $\mathrm{Na}_{2} \mathrm{SO}_{4}$, and concentrated in vacuo. Purification of the crude product by flash chromatography on silica gel using the indicated solvent system afforded the desired product. 
Representative example at $1 \mathrm{mmol}$ scale: To a $20 \mathrm{~mL}$ vial equipped with a Teflon septum and a magnetic stir bar was charged lepidine (143.2 mg, $1.0 \mathrm{mmol}, 1.0$ equiv.), Selectfluor (746.0 mg, $2.0 \mathrm{mmol}, 2.0$ equiv.), $10.0 \mathrm{~mL}$ of acetone. The reaction mixture was degassed by sparging with nitrogen for 10 min with an outlet needle, and added with hydrochloric acid ( $86.0 \mu \mathrm{l}, 1.0 \mathrm{mmol}, 1.0$ equiv.), cyclohexane ( $1.1 \mathrm{ml}, 10.0 \mathrm{mmol}, 10.0$ equiv.), then irradiated with Kessil $40 \mathrm{~W} 427 \mathrm{~nm}$ LEDs (approximately $8 \mathrm{~cm}$ away from the light source) under two mini fans at room temperature. Upon reaction completion as judged by TLC and LCMS (about 16 hours), the reaction mixture was diluted with $1.0 \mathrm{M}$ $\mathrm{NaOH}$ aqueous solution $(40 \mathrm{~mL})$, and extracted by $\mathrm{CH}_{2} \mathrm{Cl}_{2}(3 \times 40 \mathrm{~mL})$, dried over $\mathrm{Na}_{2} \mathrm{SO}_{4}$, and concentrated in vacuo. Purification of the crude product by flash chromatography (10\% ethyl acetate/hexanes) on silica gel provided 2-cyclohexyl-4methylquinoline as a colorless oil (211.9 mg, 94\% yield).

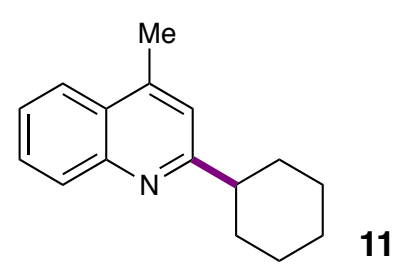

2-Cyclohexyl-4-methylquinoline (11): According to the general procedure (Conditions A), lepidine (71.6 mg, $0.50 \mathrm{mmol}, 1.0$ equiv.), Selectfluor (373.0 mg, $1.0 \mathrm{mmol}, 2.0$ equiv.), hydrochloric acid (43.0 $\mu$ l, $0.50 \mathrm{mmol}, 1.0$ equiv.), cyclohexane $(0.55 \mathrm{ml}, 5.0$ mmol, 10.0 equiv.), $5.0 \mathrm{~mL}$ of acetone were used. After 16 hours, the reaction mixture was subjected to the workup procedure outlined in the general procedure and purified by flash chromatography (10\% ethyl acetate/hexanes) to provide the title compound as a colorless oil ( $99.2 \mathrm{mg}, 88 \%$ yield). 
${ }^{1} \mathrm{H}$ NMR $\left(400 \mathrm{MHz}, \mathrm{CDCl}_{3}\right) \delta 8.06(\mathrm{~d}, J=8.4 \mathrm{~Hz}, 1 \mathrm{H}), 7.92(\mathrm{~d}, J=8.3 \mathrm{~Hz}, 1 \mathrm{H}), 7.68-$ $7.61(\mathrm{~m}, 1 \mathrm{H}), 7.51-7.44(\mathrm{~m}, 1 \mathrm{H}), 7.16(\mathrm{~s}, 1 \mathrm{H}), 2.88(\mathrm{tt}, J=12.1,3.4 \mathrm{~Hz}, 1 \mathrm{H}), 2.66(\mathrm{~s}$, $3 \mathrm{H}), 2.07-1.96(\mathrm{~m}, 2 \mathrm{H}), 1.93-1.84(\mathrm{~m}, 2 \mathrm{H}), 1.83-1.73(\mathrm{~m}, 1 \mathrm{H}), 1.69-1.55(\mathrm{~m}, 2 \mathrm{H})$, $1.55-1.40(\mathrm{~m}, 2 \mathrm{H}), 1.40-1.29(\mathrm{~m}, 1 \mathrm{H}) ;{ }^{13} \mathrm{C} \mathrm{NMR}\left(101 \mathrm{MHz}, \mathrm{CDCl}_{3}\right) \delta$ 166.35, 147.40, $144.23,129.28,128.85,126.92,125.28,123.44,120.13,47.44,32.73,26.47,26.03$, 18.74; HRMS (ESI) $\mathrm{m} / \mathrm{z}$ calculated for $\mathrm{C}_{16} \mathrm{H}_{20} \mathrm{~N}\left[(\mathrm{M}+\mathrm{H})^{+}\right]$226.1590, found 226.1589; IR (film) $2925,2850,1604,1560,1507,1448,1341,861,758 \mathrm{~cm}^{-1}$. Spectra data are consistent with those reported in the literature: Angew. Chem. Int. Ed. 2013, 52, $3267-3271$.

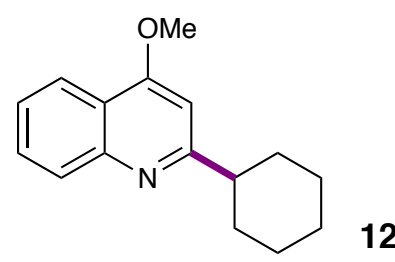

2-Cyclohexyl-4-methoxyquinoline (12): According to the general procedure (Conditions A), 4-methoxyquinoline (79.6 mg, $0.50 \mathrm{mmol}, 1.0$ equiv.), Selectfluor (466.3 mg, $1.25 \mathrm{mmol}, 2.5$ equiv.), hydrochloric acid (43.0 $\mu \mathrm{l}, 0.50 \mathrm{mmol}, 1.0$ equiv.), cyclohexane ( $1.10 \mathrm{ml}, 10.0 \mathrm{mmol}, 20.0$ equiv.), $10.0 \mathrm{~mL}$ of acetone were used. After 16 hours, the reaction mixture was subjected to the workup procedure outlined in the general procedure and purified by flash chromatography ( $10 \%$ ethyl acetate/hexanes) to provide the title compound as a colorless oil ( $88.1 \mathrm{mg}, 73 \%$ yield).

${ }^{1} \mathrm{H} \mathrm{NMR}\left(400 \mathrm{MHz}, \mathrm{CDCl}_{3}\right) \delta 8.13(\mathrm{~d}, J=8.3 \mathrm{~Hz}, 1 \mathrm{H}), 7.99(\mathrm{~d}, J=8.4 \mathrm{~Hz}, 1 \mathrm{H}), 7.69-$ $7.61(\mathrm{~m}, 1 \mathrm{H}), 7.47-7.39(\mathrm{~m}, 1 \mathrm{H}), 6.65(\mathrm{~s}, 1 \mathrm{H}), 4.04(\mathrm{~s}, 3 \mathrm{H}), 2.89$ (tt, $J=12.0,3.3 \mathrm{~Hz}$, 1H), $2.09-1.97(\mathrm{~m}, 2 \mathrm{H}), 1.95-1.84(\mathrm{~m}, 2 \mathrm{H}), 1.83-1.74(\mathrm{~d}, J=12.5 \mathrm{~Hz}, 1 \mathrm{H}), 1.69-$ $1.55(\mathrm{~m}, 2 \mathrm{H}), 1.54-1.41(\mathrm{~m}, 2 \mathrm{H}), 1.41-1.30(\mathrm{~m}, 1 \mathrm{H}) ;{ }^{13} \mathrm{C} \mathrm{NMR}\left(101 \mathrm{MHz}, \mathrm{CDCl}_{3}\right) \delta$ 
$168.11,162.52,148.52,129.61,128.28,124.75,121.53,120.23,97.91,55.46,48.14$, 32.90, 26.52, 26.08; HRMS (ESI) m/z calculated for $\mathrm{C}_{16} \mathrm{H}_{20} \mathrm{ON}\left[(\mathrm{M}+\mathrm{H})^{+}\right]$242.1539, found 242.1539; IR (film) 2926, 2850, 1620, 1597, 1507, 1448, 1421, 1355, 1110, 991, $835,765 \mathrm{~cm}^{-1}$.

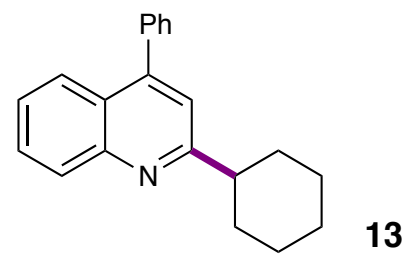

2-Cyclohexyl-4-phenylquinoline (13): According to the general procedure (Conditions A), 4-phenylquinoline (102.6 mg, $0.50 \mathrm{mmol}, 1.0$ equiv.), Selectfluor (373.0 mg, 1.0 mmol, 2.0 equiv.), hydrochloric acid (43.0 $\mu 1,0.50 \mathrm{mmol}, 1.0$ equiv.), cyclohexane ( 0.55 $\mathrm{ml}, 5.0 \mathrm{mmol}, 10.0$ equiv.), $5.0 \mathrm{~mL}$ of acetone were used. After 16 hours, the reaction mixture was subjected to the workup procedure outlined in the general procedure and purified by flash chromatography (10\% ethyl acetate/hexanes) to provide the title compound as a colorless oil $(125.0 \mathrm{mg}, 87 \%$ yield $)$.

${ }^{1} \mathrm{H}$ NMR $\left(400 \mathrm{MHz}, \mathrm{CDCl}_{3}\right) \delta 8.15(\mathrm{~d}, J=8.4 \mathrm{~Hz}, 1 \mathrm{H}), 7.87(\mathrm{~d}, J=8.4 \mathrm{~Hz}, 1 \mathrm{H}), 7.72-$ $7.64(\mathrm{~m}, 1 \mathrm{H}), 7.56-7.44$ (m, 5H), $7.44-7.39(\mathrm{~m}, 1 \mathrm{H}), 7.28(\mathrm{~s}, 1 \mathrm{H}), 2.98(\mathrm{tt}, J=12.0$ $3.4 \mathrm{~Hz}, 1 \mathrm{H}), 2.15-2.01(\mathrm{~m}, 2 \mathrm{H}), 1.96-1.85(\mathrm{~m}, 2 \mathrm{H}), 1.84-1.75(\mathrm{~m}, 1 \mathrm{H}), 1.74-1.61$ $(\mathrm{m}, 2 \mathrm{H}), 1.56-1.42(\mathrm{~m}, 2 \mathrm{H}), 1.40-1.28(\mathrm{~m}, 1 \mathrm{H}) ;{ }^{13} \mathrm{C} \mathrm{NMR}\left(101 \mathrm{MHz}, \mathrm{CDCl}_{3}\right) \delta$ $166.25,148.61,148.14,138.43,129.47,129.23,129.07,128.41,128.16,125.61,125.51$, 125.44, 119.77, 47.55, 32.81, 26.48, 26.03; HRMS (ESI) $\mathrm{m} / \mathrm{z}$ calculated for $\mathrm{C}_{21} \mathrm{H}_{22} \mathrm{~N}$ $\left[(\mathrm{M}+\mathrm{H})^{+}\right]$288.1747, found 288.1746; IR (film) 2925, 2850, 1592, 1556, 1490, 1446, 793, $701 \mathrm{~cm}^{-1}$. Spectra data are consistent with those reported in the literature: J. Am. Chem. Soc. 2017, 139, 14315-14321. 


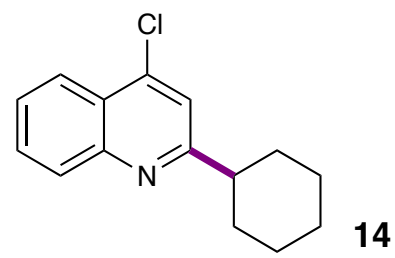

4-Chloro-2-cyclohexylquinoline (14): According to the general procedure (Conditions B), 4-chloroquinoline ( $81.8 \mathrm{mg}, 0.50 \mathrm{mmol}, 1.0$ equiv.), Selectfluor (373.0 mg, $1.0 \mathrm{mmol}$, 2.0 equiv.), trifluoroacetic acid (72.3 $\mu 1,1.0 \mathrm{mmol}, 2.0$ equiv.), cyclohexane $(1.10 \mathrm{ml}$, $10.0 \mathrm{mmol}, 20.0$ equiv.), $5.0 \mathrm{~mL}$ of acetonitrile were used. After 16 hours, the reaction mixture was subjected to the workup procedure outlined in the general procedure and purified by flash chromatography (10\% ethyl acetate/hexanes) to provide the title compound as a colorless oil (109.4 $\mathrm{mg}, 89 \%$ yield).

${ }^{1} \mathrm{H} \mathrm{NMR}\left(400 \mathrm{MHz}, \mathrm{CDCl}_{3}\right) \delta 8.17(\mathrm{~d}, J=8.3 \mathrm{~Hz}, 1 \mathrm{H}), 8.06(\mathrm{~d}, J=8.4 \mathrm{~Hz}, 1 \mathrm{H}), 7.76-$ $7.69(\mathrm{~m}, 1 \mathrm{H}), 7.60-7.53(\mathrm{~m}, 1 \mathrm{H}), 7.42(\mathrm{~s}, 1 \mathrm{H}), 2.89(\mathrm{tt}, J=12.0,3.4 \mathrm{~Hz}, 1 \mathrm{H}), 2.07-$ $1.97(\mathrm{~m}, 2 \mathrm{H}), 1.94-1.84(\mathrm{~m}, 2 \mathrm{H}), 1.83-1.73(\mathrm{~m}, 1 \mathrm{H}), 1.67-1.54(\mathrm{~m}, 2 \mathrm{H}), 1.53-1.39$ $(\mathrm{m}, 2 \mathrm{H}), 1.39-1.27(\mathrm{~m}, 1 \mathrm{H}) ;{ }^{13} \mathrm{C} \mathrm{NMR}\left(101 \mathrm{MHz}, \mathrm{CDCl}_{3}\right) \delta$ 166.76, 148.51, 142.69, $130.19,129.18,126.60,125.10,123.87,119.76,47.32,32.66,26.39,25.96 ;$ HRMS (ESI) $\mathrm{m} / \mathrm{z}$ calculated for $\mathrm{C}_{15} \mathrm{H}_{17} \mathrm{NCl}\left[(\mathrm{M}+\mathrm{H})^{+}\right]$246.1044, found 246.1043; IR (film) 2926, 2851, $1589,1493,1448,1409,839,760 \mathrm{~cm}^{-1}$. Spectra data are consistent with those reported in the literature: Org. Lett. 2018, 20, 4686-4690.

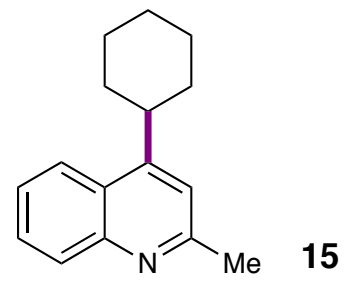

4-Cyclohexyl-2-methylquinoline (15): According to the general procedure (Conditions A), 2-methylquinoline (71.6 mg, $0.50 \mathrm{mmol}, 1.0$ equiv.), Selectfluor $(373.0 \mathrm{mg}, 1.0$ 
mmol, 2.0 equiv.), hydrochloric acid (43.0 $\mu 1,0.50 \mathrm{mmol}, 1.0$ equiv.), cyclohexane (1.10 $\mathrm{ml}, 10.0 \mathrm{mmol}, 20.0$ equiv.), $5.0 \mathrm{~mL}$ of acetone were used. After 16 hours, the reaction mixture was subjected to the workup procedure outlined in the general procedure and purified by flash chromatography (10\% ethyl acetate/hexanes) to provide the title compound as a colorless oil ( $87.9 \mathrm{mg}, 78 \%$ yield).

${ }^{1} \mathrm{H}$ NMR $\left(400 \mathrm{MHz}, \mathrm{CDCl}_{3}\right) \delta 8.03(\mathrm{~d}, J=7.6 \mathrm{~Hz}, 1 \mathrm{H}), 8.02(\mathrm{~d}, J=7.1 \mathrm{~Hz}, 1 \mathrm{H}), 7.63$ (ddd, $J=8.3,6.8,1.4 \mathrm{~Hz}, 1 \mathrm{H}), 7.47$ (ddd, $J=8.3,6.9,1.3 \mathrm{~Hz}, 1 \mathrm{H}), 7.15(\mathrm{~s}, 1 \mathrm{H}), 3.27$ (tt, $J=8.5,3.0 \mathrm{~Hz}, 1 \mathrm{H}), 2.71(\mathrm{~s}, 3 \mathrm{H}), 2.06-1.95(\mathrm{~m}, 2 \mathrm{H}), 1.95-1.87(\mathrm{~m}, 2 \mathrm{H}), 1.87-1.77$ $(\mathrm{m}, 1 \mathrm{H}), 1.61-1.44(\mathrm{~m}, 4 \mathrm{H}), 1.38-1.26(\mathrm{~m}, 1 \mathrm{H}) ;{ }^{13} \mathrm{C} \mathrm{NMR}\left(101 \mathrm{MHz}, \mathrm{CDCl}_{3}\right) \delta$ $158.68,153.37,147.91,129.33,128.76,125.23,125.07,122.76,118.25,38.71,33.48$, 26.85, 26.24, 25.37; HRMS (ESI) $\mathrm{m} / \mathrm{z}$ calculated for $\mathrm{C}_{16} \mathrm{H}_{20} \mathrm{~N}\left[(\mathrm{M}+\mathrm{H})^{+}\right] 226.1590$, found 226.1590; IR (film) 2927, 2852, 1601, 1563, 1510, 1448, 1413, 1028, 758, $732 \mathrm{~cm}^{-1}$. Spectra data are consistent with those reported in the literature: Angew. Chem. Int. Ed. 2013, 52, 3267-3271.

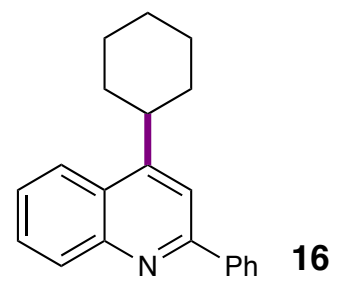

4-Cyclohexyl-2-phenylquinoline (16): According to the general procedure (Conditions A), 2-phenylquinoline (102.6 mg, $0.50 \mathrm{mmol}, 1.0$ equiv.), Selectfluor (410.3 mg, 1.1 mmol, 2.2 equiv.), hydrochloric acid ( $43.0 \mu 1,0.50 \mathrm{mmol}, 1.0$ equiv.), cyclohexane (1.10 $\mathrm{ml}, 10.0 \mathrm{mmol}, 20.0$ equiv.), $5.0 \mathrm{~mL}$ of acetone were used. After 16 hours, the reaction mixture was subjected to the workup procedure outlined in the general procedure and 
purified by flash chromatography (10\% ethyl acetate/hexanes) to provide the title compound as a white solid (102.0 $\mathrm{mg}, 71 \%$ yield).

${ }^{1} \mathrm{H}$ NMR $\left(400 \mathrm{MHz}, \mathrm{CDCl}_{3}\right) \delta 8.23(\mathrm{~d}, J=8.4 \mathrm{~Hz}, 1 \mathrm{H}), 8.16(\mathrm{~d}, J=7.2 \mathrm{~Hz}, 2 \mathrm{H}), 8.10(\mathrm{~d}$, $J=8.4 \mathrm{~Hz}, 1 \mathrm{H}), 7.77(\mathrm{~s}, 1 \mathrm{H}), 7.71(\mathrm{t}, J=7.6 \mathrm{~Hz}, 1 \mathrm{H}), 7.54(\mathrm{t}, J=7.6 \mathrm{~Hz}, 3 \mathrm{H}), 7.47$ (t, $J$

$=7.3 \mathrm{~Hz}, 1 \mathrm{H}), 3.39(\mathrm{tt}, J=11.5,3.0 \mathrm{~Hz}, 1 \mathrm{H}), 2.14-2.04(\mathrm{~m}, 2 \mathrm{H}), 2.01-1.92(\mathrm{~m}, 2 \mathrm{H})$, $1.91-1.83(\mathrm{~m}, 1 \mathrm{H}), 1.70-1.51(\mathrm{~m}, 4 \mathrm{H}), 1.45-1.32(\mathrm{~m}, 1 \mathrm{H}) ;{ }^{13} \mathrm{C}$ NMR $(101 \mathrm{MHz}$ $\left.\mathrm{CDCl}_{3}\right) \delta 157.27,154.03,148.42,140.12,130.55,129.11,129.01,128.73,127.60$, 125.87, 125.82, 122.80, 115.49, 39.07, 33.61, 26.91, 26.27; HRMS (ESI) $\mathrm{m} / \mathrm{z}$ calculated for $\mathrm{C}_{21} \mathrm{H}_{22} \mathrm{~N}\left[(\mathrm{M}+\mathrm{H})^{+}\right]$288.1747, found 288.1747; IR (film) 2927, 2851, 1593, 1551, $1490,1446,1412,877,769,694 \mathrm{~cm}^{-1}$. Spectra data are consistent with those reported in the literature: Angew. Chem. Int. Ed. 2013, 52, 3267-3271.

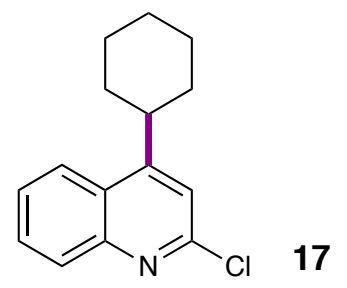

2-Chloro-4-cyclohexylquinoline (17): According to the general procedure (Conditions B), 2-chloroquinoline ( $81.8 \mathrm{mg}, 0.50 \mathrm{mmol}, 1.0$ equiv.), Selectfluor (410.3 mg, $1.1 \mathrm{mmol}$, 2.2 equiv.), trifluoroacetic acid ( $72.3 \mu \mathrm{l}, 1.0 \mathrm{mmol}, 2.0$ equiv.), cyclohexane (1.10 ml, $10.0 \mathrm{mmol}, 20.0$ equiv.), $5.0 \mathrm{~mL}$ of acetonitrile were used. After 16 hours, the reaction mixture was subjected to the workup procedure outlined in the general procedure and purified by flash chromatography ( $10 \%$ ethyl acetate/hexanes) to provide the title compound as a white solid ( $106.9 \mathrm{mg}, 87 \%$ yield).

${ }^{1} \mathrm{H}$ NMR $\left(400 \mathrm{MHz}, \mathrm{CDCl}_{3}\right) \delta 8.03(\mathrm{~d}, J=7.4 \mathrm{~Hz}, 1 \mathrm{H}), 8.01(\mathrm{~d}, J=7.4 \mathrm{~Hz}, 1 \mathrm{H}), 7.69(\mathrm{t}$, $J=7.6 \mathrm{~Hz}, 1 \mathrm{H}), 7.55(\mathrm{t}, J=7.6 \mathrm{~Hz}, 1 \mathrm{H}), 7.25(\mathrm{~s}, 1 \mathrm{H}), 3.28(\mathrm{tt}, J=11.5,3.2 \mathrm{~Hz}, 1 \mathrm{H}), 2.07$ 
$-1.96(\mathrm{~m}, 2 \mathrm{H}), 1.96-1.88(\mathrm{~m}, 2 \mathrm{H}), 1.88-1.80(\mathrm{~m}, 1 \mathrm{H}), 1.60-1.43(\mathrm{~m}, 4 \mathrm{H}), 1.38-$ $1.26(\mathrm{~m}, 1 \mathrm{H}) ;{ }^{13} \mathrm{C} \mathrm{NMR}\left(101 \mathrm{MHz}, \mathrm{CDCl}_{3}\right) \delta 156.82,150.98,148.07,129.83,129.38$, $126.42,125.50,123.06,118.59,38.98,33.29,26.67,26.04 ;$ HRMS (EI) $\mathrm{m} / \mathrm{z}$ calculated for $\mathrm{C}_{15} \mathrm{H}_{16} \mathrm{ClN}\left[\mathrm{M}^{+}\right]$245.0971, found 245.0974; IR (film) 2929, 2850, 1596, 1577, 1553, $1446,1418,866,773,696 \mathrm{~cm}^{-1}$. Spectra data are consistent with those reported in the literature: Angew. Chem. Int. Ed. 2013, 52, 3267-3271.

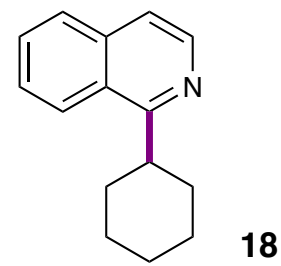

1-Cyclohexylisoquinoline (18): According to the general procedure (Conditions A), isoquinoline (64.6 mg, $0.50 \mathrm{mmol}, 1.0$ equiv.), Selectfluor (373.0 mg, $1.0 \mathrm{mmol}, 2.0$ equiv.), hydrochloric acid (43.0 $\mu \mathrm{l}, 0.50 \mathrm{mmol}, 1.0$ equiv.), cyclohexane (1.10 ml, 10.0 mmol, 20.0 equiv.), $10.0 \mathrm{~mL}$ of acetone were used. After 16 hours, the reaction mixture was subjected to the workup procedure outlined in the general procedure and purified by flash chromatography ( $10 \%$ ethyl acetate/hexanes) to provide the title compound as a colorless oil ( $80.3 \mathrm{mg}, 76 \%$ yield).

${ }^{1} \mathrm{H}$ NMR $\left(400 \mathrm{MHz}, \mathrm{CDCl}_{3}\right) \delta 8.48(\mathrm{~d}, J=5.7 \mathrm{~Hz}, 1 \mathrm{H}), 8.21(\mathrm{~d}, J=8.4 \mathrm{~Hz}, 1 \mathrm{H}), 7.79(\mathrm{~d}$, $J=8.1 \mathrm{~Hz}, 1 \mathrm{H}), 7.66-7.60(\mathrm{~m}, 1 \mathrm{H}), 7.60-7.53(\mathrm{~m}, 1 \mathrm{H}), 7.46(\mathrm{~d}, J=5.7 \mathrm{~Hz}, 1 \mathrm{H}), 3.56$ (tt, $J=11.7,3.2 \mathrm{~Hz}, 1 \mathrm{H}), 2.03-1.90(\mathrm{~m}, 4 \mathrm{H}), 1.90-1.77(\mathrm{~m}, 3 \mathrm{H}), 1.60-1.47(\mathrm{~m}, 2 \mathrm{H})$, $1.45-1.33(\mathrm{~m}, 1 \mathrm{H}) ;{ }^{13} \mathrm{C}$ NMR $\left(101 \mathrm{MHz}, \mathrm{CDCl}_{3}\right) \delta 165.59,141.77,136.30,129.47$, $127.45,126.73,126.18,124.64,118.82,41.45,32.51,26.81,26.18$; HRMS (ESI) m/z calculated for $\mathrm{C}_{15} \mathrm{H}_{18} \mathrm{~N}\left[(\mathrm{M}+\mathrm{H})^{+}\right]$212.1434, found 212.1433; IR (film) 3050, 2925, 2851, 
$1621,1561,1501,1449,1390,1357,992,821,743,675 \mathrm{~cm}^{-1}$. Spectra data are consistent with those reported in the literature: Angew. Chem. Int. Ed. 2013, 52, 3267-3271.<smiles>N#Cc1cc2ccccc2c(C2CCCCC2)n1</smiles>

1-Cyclohexylisoquinoline-3-carbonitrile (19): According to the general procedure (Conditions B), isoquinoline-3-carbonitrile (77.1 mg, $0.50 \mathrm{mmol}, 1.0$ equiv.), Selectfluor (373.0 mg, $1.0 \mathrm{mmol}, 2.0$ equiv.), trifluoroacetic acid (72.3 $\mu \mathrm{l}, 1.0 \mathrm{mmol}, 2.0$ equiv.), cyclohexane (1.10 ml, $10.0 \mathrm{mmol}, 20.0$ equiv.), $5.0 \mathrm{~mL}$ of acetonitrile were used. After 16 hours, the reaction mixture was subjected to the workup procedure outlined in the general procedure and purified by flash chromatography (10\% ethyl acetate/hexanes) to provide the title compound as a white solid $(95.7 \mathrm{mg}, 81 \%$ yield).

${ }^{1} \mathrm{H}$ NMR (400 MHz, $\left.\mathrm{CDCl}_{3}\right) \delta 8.31-8.24(\mathrm{~m}, 1 \mathrm{H}), 7.95(\mathrm{~s}, 1 \mathrm{H}), 7.90-7.83(\mathrm{~m}, 1 \mathrm{H})$, $7.81-7.72(\mathrm{~m}, 2 \mathrm{H}), 3.52(\mathrm{tt}, J=11.5,3.3 \mathrm{~Hz}, 1 \mathrm{H}), 1.99-1.88(\mathrm{~m}, 4 \mathrm{H}), 1.86-1.75(\mathrm{~m}$, $3 \mathrm{H}), 1.57-1.44(\mathrm{~m}, 2 \mathrm{H}), 1.43-1.31(\mathrm{~m}, 1 \mathrm{H}) ;{ }^{13} \mathrm{C} \mathrm{NMR}\left(101 \mathrm{MHz}, \mathrm{CDCl}_{3}\right) \delta 167.79$, $135.08,130.93,129.86,128.00,127.19,125.98,125.91,124.97,118.58,41.68,32.18$, 26.55, 25.96; HRMS (ESI) $\mathrm{m} / \mathrm{z}$ calculated for $\mathrm{C}_{16} \mathrm{H}_{17} \mathrm{~N}_{2}\left[(\mathrm{M}+\mathrm{H})^{+}\right]$237.1386, found 237.1386; IR (film) 2934, 2851, 2226, 1559, 1444, 995, 901, $749 \mathrm{~cm}^{-1}$.<smiles>CC(=O)c1cc2ccccc2c(C2CCCCC2)n1</smiles>
20

Methyl 1-cyclohexylisoquinoline-3-carboxylate (20): According to the general procedure (Conditions A), methyl isoquinoline-3-carboxylate $(93.6 \mathrm{mg}, 0.50 \mathrm{mmol}, 1.0$ 
equiv.), Selectfluor (373.0 mg, $1.0 \mathrm{mmol}, 2.0$ equiv.), hydrochloric acid (43.0 $\mu 1,0.50$ mmol, 1.0 equiv.), cyclohexane ( $1.10 \mathrm{ml}, 10.0 \mathrm{mmol}, 20.0$ equiv.), $5.0 \mathrm{~mL}$ of acetone were used. After 16 hours, the reaction mixture was subjected to the workup procedure outlined in the general procedure and purified by flash chromatography $(10 \%$ ethyl acetate/hexanes) to provide the title compound as a white solid (111.8 $\mathrm{mg}, 83 \%$ yield).

${ }^{1} \mathrm{H} \mathrm{NMR}\left(400 \mathrm{MHz}, \mathrm{CDCl}_{3}\right) \delta 8.39(\mathrm{~s}, 1 \mathrm{H}), 8.30-8.24(\mathrm{~m}, 1 \mathrm{H}), 7.98-7.89(\mathrm{~m}, 1 \mathrm{H})$, $7.75-7.67(\mathrm{~m}, 2 \mathrm{H}), 4.02(\mathrm{~s}, 3 \mathrm{H}), 3.57(\mathrm{tt}, J=10.9,4.0 \mathrm{~Hz}, 1 \mathrm{H}), 2.05-1.88(\mathrm{~m}, 6 \mathrm{H})$, $1.85-1.77(\mathrm{~m}, 1 \mathrm{H}), 1.60-1.47(\mathrm{~m}, 2 \mathrm{H}), 1.47-1.38(\mathrm{~m}, 1 \mathrm{H}) ;{ }^{13} \mathrm{C}$ NMR (101 MHz, $\left.\mathrm{CDCl}_{3}\right) \delta 166.82,166.07,140.58,135.96,130.07,129.00,128.98,127.70,124.91$, $122.38,52.62,42.00,32.17,26.72,26.01 ; \mathrm{HRMS}$ (ESI) $\mathrm{m} / \mathrm{z}$ calculated for $\mathrm{C}_{17} \mathrm{H}_{20} \mathrm{O}_{2} \mathrm{~N}$ $\left[(\mathrm{M}+\mathrm{H})^{+}\right]$270.1489, found 270.1488; IR (film) 2936, 2850, 1711, 1451, 1242, 1204, 999, $749 \mathrm{~cm}^{-1}$. Spectra data are consistent with those reported in the literature: J. Am. Chem. Soc. 2008, 130, 1558-1559.<smiles>COc1cnc(C2CCCCC2)c2ccccc12</smiles>

1-Cyclohexyl-4-methoxyisoquinoline (21): According to the general procedure (Conditions A), 4-methoxyisoquinoline (79.6 mg, $0.50 \mathrm{mmol}, 1.0$ equiv.), Selectfluor (373.0 mg, $1.0 \mathrm{mmol}, 2.0$ equiv.), hydrochloric acid (43.0 $\mu 1,0.50 \mathrm{mmol}, 1.0$ equiv.), cyclohexane ( $0.55 \mathrm{ml}, 5.0 \mathrm{mmol}, 10.0$ equiv.), $5.0 \mathrm{~mL}$ of acetone were used. After 16 hours, the reaction mixture was subjected to the workup procedure outlined in the general 
procedure and purified by flash chromatography (10\% ethyl acetate/hexanes) to provide the title compound as a colorless oil ( $77.2 \mathrm{mg}, 64 \%$ yield).

${ }^{1} \mathrm{H}$ NMR (400 MHz, $\left.\mathrm{CDCl}_{3}\right) \delta 8.22(\mathrm{~d}, J=8.2 \mathrm{~Hz}, 1 \mathrm{H}), 8.15(\mathrm{~d}, J=8.4 \mathrm{~Hz}, 1 \mathrm{H}), 8.04(\mathrm{~s}$, 1H), 7.65 (t, $J=7.6 \mathrm{~Hz}, 1 \mathrm{H}), 7.59$ (t, $J=7.6 \mathrm{~Hz}, 1 \mathrm{H}), 4.03$ (s, 3H), 3.45 (tt, $J=11.6,3.2$ Hz, 1H), $2.01-1.88(\mathrm{~m}, 4 \mathrm{H}), 1.87-1.72(\mathrm{~m}, 3 \mathrm{H}), 1.60-1.45(\mathrm{~m}, 2 \mathrm{H}), 1.45-1.31(\mathrm{~m}$, $1 \mathrm{H}) ;{ }^{13} \mathrm{C}$ NMR $\left(101 \mathrm{MHz}, \mathrm{CDCl}_{3}\right) \delta 157.98,148.80,128.80,128.71,127.09,126.47$, 124.33, 121.71, 121.53, 55.82, 41.04, 32.61, 26.90, 26.26; HRMS (ESI) m/z calculated for $\mathrm{C}_{16} \mathrm{H}_{20} \mathrm{ON}\left[(\mathrm{M}+\mathrm{H})^{+}\right]$242.1539, found 242.1539; IR (film) 2923, 2846, 1582, 1464, $1393,1321,1282,1103,998,864,760 \mathrm{~cm}^{-1}$.<smiles>O=C1CCCCC1c1ncc(Br)c2ccccc12</smiles>

4-Bromo-1-cyclohexylisoquinoline (22): According to the general procedure (Conditions B), 4-bromoisoquinoline (104.0 mg, $0.50 \mathrm{mmol}$, 1.0 equiv.), Selectfluor (373.0 mg, $1.0 \mathrm{mmol}, 2.0$ equiv.), trifluoroacetic acid (72.3 $\mu \mathrm{l}, 1.0 \mathrm{mmol}, 2.0$ equiv.), cyclohexane (1.10 ml, $10.0 \mathrm{mmol}, 20.0$ equiv.), $5.0 \mathrm{~mL}$ of acetonitrile were used. After 16 hours, the reaction mixture was subjected to the workup procedure outlined in the general procedure and purified by flash chromatography (10\% ethyl acetate/hexanes) to provide the title compound as a colorless oil ( $117.5 \mathrm{mg}, 81 \%$ yield).

${ }^{1} \mathrm{H}$ NMR $\left(400 \mathrm{MHz}, \mathrm{CDCl}_{3}\right) \delta 8.65(\mathrm{~s}, 1 \mathrm{H}), 8.21(\mathrm{~d}, J=8.5 \mathrm{~Hz}, 1 \mathrm{H}), 8.18(\mathrm{~d}, J=8.5 \mathrm{~Hz}$, 1H), $7.76(\mathrm{t}, J=7.6 \mathrm{~Hz}, 1 \mathrm{H}), 7.64(\mathrm{t}, J=7.7 \mathrm{~Hz}, 1 \mathrm{H}), 3.51(\mathrm{tt}, J=11.7,3.0 \mathrm{~Hz}, 1 \mathrm{H}), 2.01$ $-1.87(\mathrm{~m}, 4 \mathrm{H}), 1.87-1.72(\mathrm{~m}, 3 \mathrm{H}), 1.59-1.45(\mathrm{~m}, 2 \mathrm{H}), 1.45-1.31(\mathrm{~m}, 1 \mathrm{H}) ;{ }^{13} \mathrm{C}$ NMR 
$\left(101 \mathrm{MHz}, \mathrm{CDCl}_{3}\right) \delta 165.26,143.30,134.93,130.91,127.78,127.53,126.85,125.10$, 117.55, 41.44, 32.50, 26.75, 26.10; HRMS (ESI) m/z calculated for $\mathrm{C}_{15} \mathrm{H}_{17} \mathrm{NBr}\left[(\mathrm{M}+\mathrm{H})^{+}\right]$ 290.0539, found 290.0538; IR (film) 2937, 2845, 1566, 1496, 1357, 991, 931, $767 \mathrm{~cm}^{-1}$. Spectra data are consistent with those reported in the literature: Adv. Synth. Catal. 2015, $357,2055-2060$.<smiles>O=[N+]([O-])c1cccc2c(C3CCCCC3)nccc12</smiles>

1-Cyclohexyl-5-nitroisoquinoline (23): According to the general procedure (Conditions A), 5-nitroisoquinoline ( $87.1 \mathrm{mg}, 0.50 \mathrm{mmol}, 1.0$ equiv.), Selectfluor (466.3 mg, 1.25 mmol, 2.5 equiv.), hydrochloric acid ( $43.0 \mu 1,0.50 \mathrm{mmol}, 1.0$ equiv.), cyclohexane (1.10 $\mathrm{ml}, 10.0 \mathrm{mmol}, 20.0$ equiv.), $10.0 \mathrm{~mL}$ of acetone were used. After 16 hours, the reaction mixture was subjected to the workup procedure outlined in the general procedure and purified by flash chromatography (20\% ethyl acetate/hexanes) to provide the title compound as a colorless oil ( $62.8 \mathrm{mg}, 49 \%$ yield).

${ }^{1} \mathrm{H} \mathrm{NMR}\left(400 \mathrm{MHz}, \mathrm{CDCl}_{3}\right) \delta 8.67(\mathrm{~d}, J=6.2 \mathrm{~Hz}, 1 \mathrm{H}), 8.57(\mathrm{~d}, J=8.6 \mathrm{~Hz}, 1 \mathrm{H}), 8.41(\mathrm{~d}$, $J=7.7 \mathrm{~Hz}, 1 \mathrm{H}), 8.20(\mathrm{~d}, J=6.2 \mathrm{~Hz}, 1 \mathrm{H}), 7.67$ (t, $J=8.1 \mathrm{~Hz}, 1 \mathrm{H}), 3.56$ (tt, $J=11.5,3.1$ Hz, 1H), $2.01-1.90(\mathrm{~m}, 4 \mathrm{H}), 1.89-1.79(\mathrm{~m}, 3 \mathrm{H}), 1.60-1.46(\mathrm{~m}, 2 \mathrm{H}), 1.44-1.35(\mathrm{~m}$, $1 \mathrm{H}) ;{ }^{13} \mathrm{C}$ NMR $\left(101 \mathrm{MHz}, \mathrm{CDCl}_{3}\right) \delta 166.41,145.96,145.16,131.36,128.80,127.21$, $126.80,125.10,113.39,42.15,32.69,26.70,26.05$; HRMS (ESI) $\mathrm{m} / \mathrm{z}$ calculated for $\mathrm{C}_{15} \mathrm{H}_{17} \mathrm{~N}_{2} \mathrm{O}_{2}\left[(\mathrm{M}+\mathrm{H})^{+}\right]$257.1285, found 257.1285; IR (film) 2928, 2853, 1624, 1556, 
$1525,1449,1332,820,736 \mathrm{~cm}^{-1}$. Spectra data are consistent with those reported in the literature: Angew. Chem. Int. Ed. 2013, 52, 3267-3271.

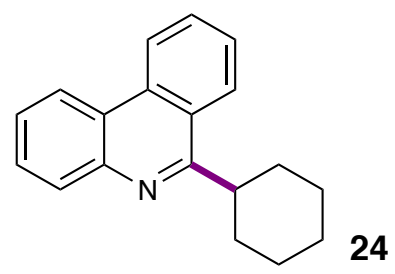

6-Cyclohexylphenanthridine (24): According to the general procedure (Conditions A), phenanthridine ( $89.6 \mathrm{mg}, 0.50 \mathrm{mmol}, 1.0$ equiv.), Selectfluor $(373.0 \mathrm{mg}, 1.0 \mathrm{mmol}, 2.0$ equiv.), hydrochloric acid (43.0 $\mu \mathrm{l}, 0.50 \mathrm{mmol}, 1.0$ equiv.), cyclohexane $(0.82 \mathrm{ml}, 7.50$ mmol, 15.0 equiv.), $5.0 \mathrm{~mL}$ of acetone were used. After 16 hours, the reaction mixture was subjected to the workup procedure outlined in the general procedure and purified by flash chromatography (5\% ethyl acetate/hexanes) to provide the title compound as a colorless oil (113.7 mg, 87\% yield).

${ }^{1} \mathrm{H}$ NMR $\left(400 \mathrm{MHz}, \mathrm{CDCl}_{3}\right) \delta 8.65(\mathrm{~d}, J=8.2 \mathrm{~Hz}, 1 \mathrm{H}), 8.54(\mathrm{~d}, J=8.0 \mathrm{~Hz}, 1 \mathrm{H}), 8.32(\mathrm{~d}$, $J=8.2 \mathrm{~Hz}, 1 \mathrm{H}), 8.15(\mathrm{~d}, J=8.1 \mathrm{~Hz}, 1 \mathrm{H}), 7.81(\mathrm{t}, J=7.3 \mathrm{~Hz}, 1 \mathrm{H}), 7.70$ (q, $J=8.0,7.4$ $\mathrm{Hz}, 2 \mathrm{H}), 7.60(\mathrm{t}, J=7.6 \mathrm{~Hz}, 1 \mathrm{H}), 3.62(\mathrm{tt}, J=11.2,3.4 \mathrm{~Hz}, 1 \mathrm{H}), 2.15-2.04(\mathrm{~m}, 2 \mathrm{H})$, $2.03-1.89(\mathrm{~m}, 4 \mathrm{H}), 1.89-1.81(\mathrm{~m}, 1 \mathrm{H}), 1.65-1.51(\mathrm{~m}, 2 \mathrm{H}), 1.51-1.39(\mathrm{~m}, 1 \mathrm{H}) ;{ }^{13} \mathrm{C}$ NMR $\left(101 \mathrm{MHz}, \mathrm{CDCl}_{3}\right) \delta 165.28,143.84,133.01,129.92,129.88,128.37,127.05$, $126.12,125.61,124.68,123.32,122.55,121.78,41.96,32.26,26.85,26.28$; HRMS (ESI) $\mathrm{m} / \mathrm{z}$ calculated for $\mathrm{C}_{19} \mathrm{H}_{20} \mathrm{~N}\left[(\mathrm{M}+\mathrm{H})^{+}\right]$262.1590, found 262.1590; IR (film) 2926, 2850, $1581,1486,1448,988,757,726 \mathrm{~cm}^{-1}$. Spectra data are consistent with those reported in the literature: J. Org. Chem. 2018, 83, 10015-10024. 
<smiles>[R5]c1cccc2c(C3CCCCC3)nnc(C3CCCCC3)c12</smiles>

1,4-Dicyclohexylphthalazine (25): According to the general procedure (Conditions B), phthalazine (65.1 mg, $0.50 \mathrm{mmol}, 1.0$ equiv.), Selectfluor (466.3 mg, $1.25 \mathrm{mmol}, 2.5$ equiv.), trifluoroacetic acid (72.3 $\mu \mathrm{l}, 1.0 \mathrm{mmol}, 2.0$ equiv.), cyclohexane (1.10 ml, 10.0 mmol, 20.0 equiv.), $10.0 \mathrm{~mL}$ of acetonitrile were used. After 16 hours, the reaction mixture was subjected to the workup procedure outlined in the general procedure and purified by flash chromatography (20\% ethyl acetate/hexanes) to provide the title compound as a yellow solid (92.6 mg, 63\% yield).

${ }^{1} \mathrm{H}$ NMR $\left(400 \mathrm{MHz}, \mathrm{CDCl}_{3}\right) \delta 8.11-8.03(\mathrm{~m}, 2 \mathrm{H}), 7.78-7.71(\mathrm{~m}, 2 \mathrm{H}), 3.38(\mathrm{tt}, J=$ 11.2, $3.4 \mathrm{~Hz}, 2 \mathrm{H}), 2.01-1.79(\mathrm{~m}, 12 \mathrm{H}), 1.78-1.66(\mathrm{~m}, 2 \mathrm{H}), 1.51-1.36(\mathrm{~m}, 4 \mathrm{H}), 1.36-$ $1.24(\mathrm{~m}, 2 \mathrm{H}) ;{ }^{13} \mathrm{C} \mathrm{NMR}\left(101 \mathrm{MHz}, \mathrm{CDCl}_{3}\right) \delta 161.73,131.08,124.87,124.12,40.37$, 32.25, 26.81, 26.17; HRMS (ESI) m/z calculated for $\mathrm{C}_{20} \mathrm{H}_{27} \mathrm{~N}_{2}\left[(\mathrm{M}+\mathrm{H})^{+}\right] 295.2169$, found 295.2168; IR (film) 2926, 2847, 1537, 1446, 1400, 1344, 998, 984, 890, $757 \mathrm{~cm}^{-1}$. Spectra data are consistent with those reported in the literature: Angew. Chem. Int. Ed. 2013, 52, 3267-3271.<smiles>O=[PH](=O)(O)C1CCCCC1c1nc2ccccc2nc1Cl</smiles>

2-Chloro-3-cyclohexylquinoxaline (26): According to the general procedure (Conditions B), 2-chloro-quinoxaline (82.3 mg, $0.50 \mathrm{mmol}, 1.0$ equiv.), Selectfluor 
(466.3 mg, $1.25 \mathrm{mmol}, 2.5$ equiv.), trifluoroacetic acid (72.3 $\mu \mathrm{l}, 1.0 \mathrm{mmol}, 2.0$ equiv.), cyclohexane (1.10 ml, $10.0 \mathrm{mmol}, 20.0$ equiv.), $10.0 \mathrm{~mL}$ of acetonitrile were used. After 16 hours, the reaction mixture was subjected to the workup procedure outlined in the general procedure and purified by flash chromatography (10\% ethyl acetate/hexanes) to provide the title compound as a white solid $(72.8 \mathrm{mg}, 59 \%$ yield).

${ }^{1} \mathrm{H}$ NMR $\left(400 \mathrm{MHz}, \mathrm{CDCl}_{3}\right) \delta 8.07-8.01(\mathrm{~m}, 1 \mathrm{H}), 7.98-7.93(\mathrm{~m}, 1 \mathrm{H}), 7.75-7.65(\mathrm{~m}$ 2H), $3.34(\mathrm{tt}, J=11.6,3.3 \mathrm{~Hz}, 1 \mathrm{H}), 2.07-1.97(\mathrm{~m}, 2 \mathrm{H}), 1.97-1.87(\mathrm{~m}, 2 \mathrm{H}), 1.85-1.77$ $(\mathrm{m}, 1 \mathrm{H}), 1.76-1.65(\mathrm{~m}, 2 \mathrm{H}), 1.55-1.42(\mathrm{~m}, 2 \mathrm{H}), 1.41-1.30(\mathrm{~m}, 1 \mathrm{H}) ;{ }^{13} \mathrm{C}$ NMR $(101$ $\left.\mathrm{MHz}, \mathrm{CDCl}_{3}\right) \delta 159.09,147.40,141.11,140.55,129.76(2 \mathrm{C}), 128.74,128.00,42.49$, 31.20, 26.34, 25.90; HRMS (ESI) m/z calculated for $\mathrm{C}_{14} \mathrm{H}_{16} \mathrm{ClN}_{2}\left[(\mathrm{M}+\mathrm{H})^{+}\right] 247.0997$, found 247.0996; IR (film) 2936, 2851, 1560, 1446, 1311, 1276, 1174, 1053, 990, 765, $594 \mathrm{~cm}^{-1}$. Spectra data are consistent with those reported in the literature: Angew. Chem. Int. Ed. 2013, 52, 3267-3271.
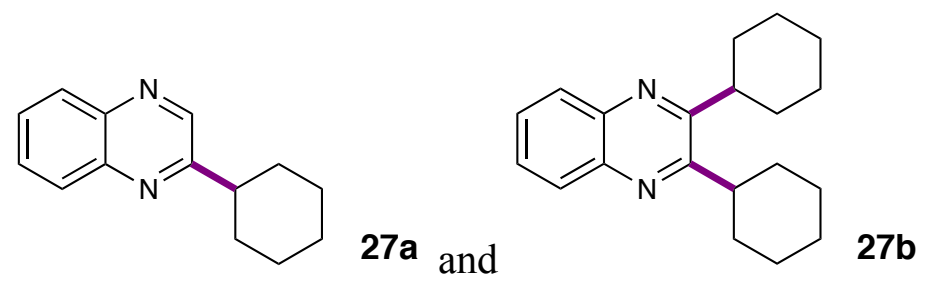

2-Cyclohexylquinoxaline (27a) and 2,3-Dicyclohexylquinoxaline (27b): According to the general procedure (Conditions B), quinoxaline (65.1 mg, $0.50 \mathrm{mmol}, 1.0$ equiv.), Selectfluor (466.3 mg, $1.25 \mathrm{mmol}, 2.5$ equiv.), trifluoroacetic acid (72.3 $\mu 1,1.0 \mathrm{mmol}, 2.0$ equiv.), cyclohexane (1.10 ml, $10.0 \mathrm{mmol}, 20.0$ equiv.), $10.0 \mathrm{~mL}$ of acetonitrile were used. After 16 hours, the reaction mixture was subjected to the workup procedure outlined in the general procedure and purified by flash chromatography $(10 \%$ ethyl 
acetate/hexanes) to provide the title compounds as a white solid (15.9 mg, 15\% yield for 27a) and a yellow solid (64.7 $\mathrm{mg}, 44 \%$ yield for $27 \mathbf{b})$.

Compound 27a: ${ }^{1} \mathrm{H}$ NMR (400 MHz, $\left.\mathrm{CDCl}_{3}\right) \delta 8.77(\mathrm{~s}, 1 \mathrm{H}), 8.07(\mathrm{~d}, J=7.8 \mathrm{~Hz}, 1 \mathrm{H})$, 8.05 (d, $J=7.9 \mathrm{~Hz}, 1 \mathrm{H}), 7.74$ (t, $J=7.8 \mathrm{~Hz}, 1 \mathrm{H}), 7.69$ (t, $J=7.8 \mathrm{~Hz}, 1 \mathrm{H}), 2.97$ (tt, $J=$ 12.0, $3.5 \mathrm{~Hz}, 1 \mathrm{H}), 2.08-2.00(\mathrm{~m}, 2 \mathrm{H}), 1.97-1.89(\mathrm{~m}, 2 \mathrm{H}), 1.85-1.77(\mathrm{~m}, 1 \mathrm{H}), 1.77-$ $1.66(\mathrm{~m}, 2 \mathrm{H}), 1.55-1.43(\mathrm{~m}, 2 \mathrm{H}), 1.41-1.32(\mathrm{~m}, 1 \mathrm{H}) ;{ }^{13} \mathrm{C} \mathrm{NMR}\left(101 \mathrm{MHz}, \mathrm{CDCl}_{3}\right) \delta$ $161.10,144.94,142.12,141.33,129.83,129.04,128.95,128.87,45.02,32.29,26.36$, 25.84; HRMS (ESI) $\mathrm{m} / \mathrm{z}$ calculated for $\mathrm{C}_{14} \mathrm{H}_{17} \mathrm{~N}_{2}\left[(\mathrm{M}+\mathrm{H})^{+}\right]$213.1386, found 213.1386; IR (film) 2926, 2852, 1559, 1491, 1448, 1126, 1108, 973, $761 \mathrm{~cm}^{-1}$. Spectra data are consistent with those reported in the literature: Chem. Commun. 2017, 53, 2335-2338. Compound 27b: ${ }^{1} \mathrm{H}$ NMR $\left(400 \mathrm{MHz}, \mathrm{CDCl}_{3}\right) \delta 7.99(\mathrm{dd}, J=6.3,3.5 \mathrm{~Hz}, 2 \mathrm{H}), 7.62(\mathrm{dd}, J$ $=6.4,3.4 \mathrm{~Hz}, 2 \mathrm{H}), 3.09(\mathrm{tt}, J=10.7,4.1 \mathrm{~Hz}, 2 \mathrm{H}), 1.97-1.76(\mathrm{~m}, 14 \mathrm{H}), 1.55-1.36(\mathrm{~m}$, $6 \mathrm{H}) ;{ }^{13} \mathrm{C} \mathrm{NMR}\left(101 \mathrm{MHz}, \mathrm{CDCl}_{3}\right) \delta 159.64,140.78,128.57,128.35,41.69,32.37,26.69$, 25.95; HRMS (ESI) $\mathrm{m} / \mathrm{z}$ calculated for $\mathrm{C}_{20} \mathrm{H}_{27} \mathrm{~N}_{2}\left[(\mathrm{M}+\mathrm{H})^{+}\right]$295.2169, found 295.2167; IR (film) 2924, 2851, 1566, 1482, 1449, 1402, 995, 760, $739 \mathrm{~cm}^{-1}$. Spectra data are consistent with those reported in the literature: Angew. Chem. Int. Ed. 2013, 52, 32673271.<smiles>Clc1nc(C2CCCCC2)c2ccccc2n1</smiles>

28

2-Chloro-4-cyclohexylquinazoline (28): According to the general procedure (Conditions A), 2-chloroquinazoline ( $82.3 \mathrm{mg}, 0.50 \mathrm{mmol}, 1.0$ equiv.), Selectfluor (410.3 mg, $1.1 \mathrm{mmol}, 2.2$ equiv.), hydrochloric acid (43.0 $\mu 1,0.50 \mathrm{mmol}, 1.0$ equiv.), 
cyclohexane ( $1.10 \mathrm{ml}, 10.0 \mathrm{mmol}, 20.0$ equiv.), $10.0 \mathrm{~mL}$ of acetone were used. After 16 hours, the reaction mixture was subjected to the workup procedure outlined in the general procedure and purified by flash chromatography (10\% ethyl acetate/hexanes) to provide the title compound as a colorless oil ( $66.6 \mathrm{mg}, 54 \%$ yield).

${ }^{1} \mathrm{H}$ NMR $\left(400 \mathrm{MHz}, \mathrm{CDCl}_{3}\right) \delta 8.08(\mathrm{~d}, J=8.4 \mathrm{~Hz}, 1 \mathrm{H}), 7.87(\mathrm{~d}, J=8.4 \mathrm{~Hz}, 1 \mathrm{H}), 7.83-$ $7.76(\mathrm{~m}, 1 \mathrm{H}), 7.58-7.50(\mathrm{~m}, 1 \mathrm{H}), 3.44(\mathrm{tt}, J=11.7,3.2 \mathrm{~Hz}, 1 \mathrm{H}), 1.91-1.81(\mathrm{~m}, 4 \mathrm{H})$ $1.81-1.69(\mathrm{~m}, 3 \mathrm{H}), 1.49-1.36(\mathrm{~m}, 2 \mathrm{H}), 1.36-1.25(\mathrm{~m}, 1 \mathrm{H}) ;{ }^{13} \mathrm{C}$ NMR (101 MHz, $\left.\mathrm{CDCl}_{3}\right) \delta 178.96,157.34,151.92,134.37,128.36,127.51,124.42,121.52,41.49,31.74$, 26.25, 25.70; HRMS (ESI) $\mathrm{m} / \mathrm{z}$ calculated for $\mathrm{C}_{14} \mathrm{H}_{16} \mathrm{~N}_{2} \mathrm{Cl}\left[(\mathrm{M}+\mathrm{H})^{+}\right]$247.0997, found 247.0996; IR (film) 2929, 2853, 1567, 1537, 1492, 1448, 1277, 1180, 921, $761 \mathrm{~cm}^{-1}$.<smiles>c1ccc2c(C3CCCCC3)ncnc2c1</smiles><smiles>c1ccc2c(C3CCCCC3)nc(C3CCCCC3)nc2c1</smiles>

29b

4-Cyclohexylquinazoline (29a) and 2,4-Dicyclohexylquinazoline (29b): According to the general procedure (Conditions B), quinazoline (65.1 $\mathrm{mg}, 0.50 \mathrm{mmol}, 1.0$ equiv.), Selectfluor (373.0 mg, $1.0 \mathrm{mmol}, 2.0$ equiv.), trifluoroacetic acid (72.3 $\mu 1,1.0 \mathrm{mmol}, 2.0$ equiv.), cyclohexane (1.10 ml, $10.0 \mathrm{mmol}, 20.0$ equiv.), $5.0 \mathrm{~mL}$ of acetonitrile were used. After 16 hours, the reaction mixture was subjected to the workup procedure outlined in the general procedure and purified by flash chromatography (10\% ethyl acetate/hexanes) to provide the title compounds as a yellow oil $(21.2 \mathrm{mg}, 20 \%$ yield for $29 a)$ and a yellow oil (91.1 mg, 62\% yield for 29b). 
Compound 29a: ${ }^{1} \mathrm{H}$ NMR (400 MHz, $\left.\mathrm{CDCl}_{3}\right) \delta 9.24(\mathrm{~s}, 1 \mathrm{H}), 8.17(\mathrm{~d}, J=8.4 \mathrm{~Hz}, 1 \mathrm{H})$, $8.03(\mathrm{~d}, J=8.4 \mathrm{~Hz}, 1 \mathrm{H}), 7.90-7.81(\mathrm{~m}, 1 \mathrm{H}), 7.66-7.58(\mathrm{~m}, 1 \mathrm{H}), 3.55(\mathrm{tt}, J=11.6,3.3$ $\mathrm{Hz}, 1 \mathrm{H}), 2.00-1.89(\mathrm{~m}, 4 \mathrm{H}), 1.88-1.74(\mathrm{~m}, 3 \mathrm{H}), 1.59-1.45(\mathrm{~m}, 2 \mathrm{H}), 1.43-1.32(\mathrm{~m}$, $1 \mathrm{H}) ;{ }^{13} \mathrm{C} \mathrm{NMR}\left(101 \mathrm{MHz}, \mathrm{CDCl}_{3}\right) \delta 175.06,154.69,150.00,133.25,129.26,127.30$, 124.13, 123.20, 41.23, 31.98, 26.47, 25.96; HRMS (ESI) m/z calculated for $\mathrm{C}_{14} \mathrm{H}_{17} \mathrm{~N}_{2}$ $\left[(\mathrm{M}+\mathrm{H})^{+}\right]$213.1386, found 213.1385; IR (film) 2928, 2852, 1615, 1568, 1557, 1495, $1449,1392,1360,994,766 \mathrm{~cm}^{-1}$. Spectra data are consistent with those reported in the literature: Angew. Chem. Int. Ed. 2013, 52, 3267-3271.

Compound 29b: ${ }^{1} \mathrm{H}$ NMR $\left(400 \mathrm{MHz}, \mathrm{CDCl}_{3}\right) \delta 8.10(\mathrm{~d}, J=8.3 \mathrm{~Hz}, 1 \mathrm{H}), 7.96(\mathrm{~d}, J=8.4$ Hz, 1H), $7.82-7.74(\mathrm{~m}, 1 \mathrm{H}), 7.55-7.47(\mathrm{~m}, 1 \mathrm{H}), 3.50(\mathrm{tt}, J=11.4,3.0 \mathrm{~Hz}, 1 \mathrm{H}), 2.98$ (tt, $J=11.6,3.4 \mathrm{~Hz}, 1 \mathrm{H}), 2.11-1.99(\mathrm{~m}, 2 \mathrm{H}), 1.97-1.72(\mathrm{~m}, 12 \mathrm{H}), 1.57-1.32(\mathrm{~m}$, $6 \mathrm{H}) ;{ }^{13} \mathrm{C} \mathrm{NMR}\left(101 \mathrm{MHz}, \mathrm{CDCl}_{3}\right) \delta 174.47,170.04,150.34,132.64,128.74,125.95$, 123.89, 121.29, 47.83, 41.25, 31.92, 31.85, 26.46, 26.28, 26.09, 26.03; HRMS (ESI) m/z calculated for $\mathrm{C}_{20} \mathrm{H}_{27} \mathrm{~N}_{2}\left[(\mathrm{M}+\mathrm{H})^{+}\right] 295.2169$, found 295.2169; IR (film) 2934, 2852, $1571,1556,1493,1449,762 \mathrm{~cm}^{-1}$. Spectra data are consistent with those reported in the literature: Angew. Chem. Int. Ed. 2013, 52, 3267-3271.

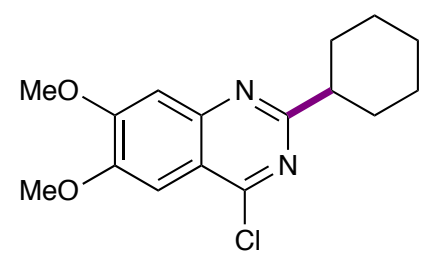

30

4-Chloro-2-cyclohexyl-6,7-dimethoxyquinazoline (30): According to the general procedure (Conditions A), 4-chloro-6,7-dimethoxyquinazoline (112.3 mg, $0.50 \mathrm{mmol}, 1.0$ equiv.), Selectfluor (410.3 mg, $1.1 \mathrm{mmol}, 2.2$ equiv.), hydrochloric acid (43.0 $\mu 1,0.50$ mmol, 1.0 equiv.), cyclohexane ( $1.10 \mathrm{ml}, 10.0 \mathrm{mmol}, 20.0$ equiv.), $5.0 \mathrm{~mL}$ of acetone 
were used. After 16 hours, the reaction mixture was subjected to the workup procedure outlined in the general procedure and purified by flash chromatography (50\% ethyl acetate/hexanes) to provide the title compound as a white solid (79.8 $\mathrm{mg}, 52 \%$ yield).

${ }^{1} \mathrm{H} \mathrm{NMR}\left(400 \mathrm{MHz}, \mathrm{CDCl}_{3}\right) \delta 7.30(\mathrm{~s}, 1 \mathrm{H}), 7.24(\mathrm{~s}, 1 \mathrm{H}), 4.01$ (s, 3H), $4.01(\mathrm{~s}, 3 \mathrm{H}), 2.89$

(tt, $J=11.9,3.5 \mathrm{~Hz}, 1 \mathrm{H}), 2.06-1.96(\mathrm{~m}, 2 \mathrm{H}), 1.88-1.78(\mathrm{~m}, 2 \mathrm{H}), 1.75-1.61(\mathrm{~m}, 3 \mathrm{H})$,

$1.46-1.26(\mathrm{~m}, 3 \mathrm{H}) ;{ }^{13} \mathrm{C} \mathrm{NMR}\left(101 \mathrm{MHz}, \mathrm{CDCl}_{3}\right) \delta 168.80,159.04,156.38,150.46$,

149.26, 117.25, 106.58, 102.63, 56.46, 56.23, 47.24, 31.75, 26.14, 25.82; HRMS (ESI)

$\mathrm{m} / \mathrm{z}$ calculated for $\mathrm{C}_{16} \mathrm{H}_{20} \mathrm{O}_{2} \mathrm{~N}_{2} \mathrm{Cl}\left[(\mathrm{M}+\mathrm{H})^{+}\right]$307.1208, found 307.1205. IR (film) 2924, $2847,1618,1570,1501,1416,1253,1162,848 \mathrm{~cm}^{-1}$.<smiles>FC(F)(F)c1ccnc(C2CCCCC2)c1</smiles>

$31 a$ and

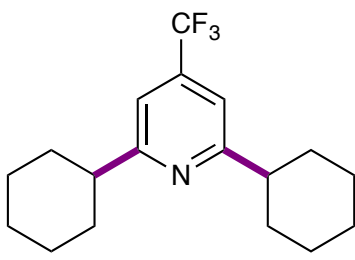

31b

(31a) and 2,6-Dicyclohexyl-4(trifluoromethyl)pyridine (31b): According to the general procedure (Conditions A), 4(trifluoromethyl)pyridine (73.6 mg, $0.50 \mathrm{mmol}, 1.0$ equiv.), Selectfluor (410.3 mg, 1.1 mmol, 2.2 equiv.), hydrochloric acid (43.0 $\mu 1,0.50 \mathrm{mmol}, 1.0$ equiv.), cyclohexane (1.10 $\mathrm{ml}, 10.0 \mathrm{mmol}, 20.0$ equiv.), $5.0 \mathrm{~mL}$ of acetone were used. After 16 hours, the reaction mixture was subjected to the workup procedure outlined in the general procedure and purified by flash chromatography ( $20 \%$ ethyl acetate/hexanes) to provide the title compounds as a colorless oil (17.2 $\mathrm{mg}, 15 \%$ yield for 31a) and a colorless oil (98.1 mg, $63 \%$ yield for $\mathbf{3 1 b})$.

Compound 31a: ${ }^{1} \mathrm{H}$ NMR (400 MHz, $\left.\mathrm{CDCl}_{3}\right) \delta 8.70(\mathrm{~d}, J=5.1 \mathrm{~Hz}, 1 \mathrm{H}), 7.36(\mathrm{~s}, 1 \mathrm{H})$, $7.32(\mathrm{~d}, J=5.0 \mathrm{~Hz}, 1 \mathrm{H}), 2.80(\mathrm{tt}, J=11.9,3.5 \mathrm{~Hz}, 1 \mathrm{H}), 2.01-1.93(\mathrm{~m}, 2 \mathrm{H}), 1.91-1.84$ 
(m, 2H), $1.80-1.73(\mathrm{~m}, 1 \mathrm{H}), 1.61-1.49(\mathrm{~m}, 2 \mathrm{H}), 1.48-1.37(\mathrm{~m}, 2 \mathrm{H}), 1.34-1.26(\mathrm{~m}$, $1 \mathrm{H}) ;{ }^{13} \mathrm{C}$ NMR (101 MHz, $\left.\mathrm{CDCl}_{3}\right) \delta$ 168.00, 149.84, 138.72 (q), 122.48 (q), 116.79 (q), 116.71 (q), 46.48, 32.72, 26.37, 25.88; HRMS (ESI) $\mathrm{m} / \mathrm{z}$ calculated for $\mathrm{C}_{12} \mathrm{H}_{15} \mathrm{NF}_{3}$ $\left[(\mathrm{M}+\mathrm{H})^{+}\right]$230.1151, found 230.1151; IR (film) 2929, 2854, 1411, 1335, 1169, 1139, 842, $667 \mathrm{~cm}^{-1}$.

Compound 31b: ${ }^{1} \mathrm{H}$ NMR (400 MHz, $\left.\mathrm{CDCl}_{3}\right) \delta 7.15$ (s, $\left.2 \mathrm{H}\right), 2.75$ (tt, $J=11.8,3.5 \mathrm{~Hz}$, 2H), $2.02-1.92(\mathrm{~m}, 4 \mathrm{H}), 1.90-1.81(\mathrm{~m}, 4 \mathrm{H}), 1.80-1.71(\mathrm{~m}, 2 \mathrm{H}), 1.56-1.35(\mathrm{~m}, 8 \mathrm{H})$, $1.35-1.24(\mathrm{~m}, 2 \mathrm{H}) ;{ }^{13} \mathrm{C} \mathrm{NMR}\left(101 \mathrm{MHz}, \mathrm{CDCl}_{3}\right) \delta 167.14,138.70$ (q), 123.37 (q), 113.50 (q), 46.55, 32.82, 26.42, 26.01; HRMS (ESI) $\mathrm{m} / \mathrm{z}$ calculated for $\mathrm{C}_{18} \mathrm{H}_{25} \mathrm{NF}_{3}$ $\left[(\mathrm{M}+\mathrm{H})^{+}\right]$312.1934, found 312.1933. IR (film) 2928, 2853, 1577, 1450, 1421, 1378, $1347,1240,1166,1138,870,676 \mathrm{~cm}^{-1}$. Spectra data are consistent with those reported in the literature: Org. Lett. 2017, 19, 6463-6465.
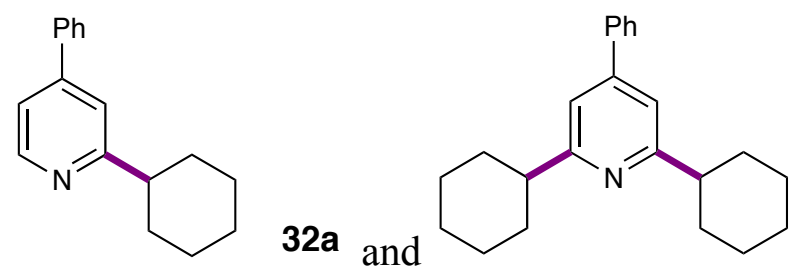

$32 b$

2-Cyclohexyl-4-phenylpyridine (32a) and 2,6-Dicyclohexyl-4-phenylpyridine (32b): According to the general procedure (Conditions A), 4-phenylpyridine (77.6 mg, 0.50 mmol, 1.0 equiv.), Selectfluor (466.3 mg, $1.25 \mathrm{mmol}, 2.5$ equiv.), hydrochloric acid (43.0 $\mu \mathrm{l}, 0.50 \mathrm{mmol}, 1.0$ equiv.), cyclohexane ( $1.10 \mathrm{ml}, 10.0 \mathrm{mmol}, 20.0$ equiv.), $10.0 \mathrm{~mL}$ of acetone were used. After 16 hours, the reaction mixture was subjected to the workup procedure outlined in the general procedure and purified by flash chromatography $(10 \%$ ethyl acetate/hexanes) to provide the title compounds as a colorless oil $(35.6 \mathrm{mg}, 30 \%$ yield for 32a) and a colorless oil (12.8 $\mathrm{mg}, 8 \%$ yield for $\mathbf{3 2 b}$ ). 
Compound 32a: ${ }^{1} \mathrm{H}$ NMR $\left(400 \mathrm{MHz}, \mathrm{CDCl}_{3}\right) \delta 8.57(\mathrm{~d}, J=5.2 \mathrm{~Hz}, 1 \mathrm{H}), 7.63(\mathrm{~d}, J=7.0$ $\mathrm{Hz}, 2 \mathrm{H}), 7.48(\mathrm{t}, J=7.2 \mathrm{~Hz}, 2 \mathrm{H}), 7.42(\mathrm{t}, J=7.1 \mathrm{~Hz}, 1 \mathrm{H}), 7.36(\mathrm{~s}, 1 \mathrm{H}), 7.32(\mathrm{dd}, J=5.2$, $1.7 \mathrm{~Hz}, 1 \mathrm{H}), 2.78(\mathrm{tt}, J=12.0,3.4 \mathrm{~Hz}, 1 \mathrm{H}), 2.05-1.94(\mathrm{~m}, 2 \mathrm{H}), 1.93-1.83(\mathrm{~m}, 2 \mathrm{H})$, $1.81-1.72(\mathrm{~m}, 1 \mathrm{H}), 1.66-1.52(\mathrm{~m}, 2 \mathrm{H}), 1.51-1.38(\mathrm{~m}, 2 \mathrm{H}), 1.37-1.25(\mathrm{~m}, 1 \mathrm{H}) ;{ }^{13} \mathrm{C}$ NMR $\left(101 \mathrm{MHz}, \mathrm{CDCl}_{3}\right) \delta 166.89,149.27,148.94,138.68,128.98,128.81,127.03$, 119.21, 119.08, 46.59, 32.96, 26.57, 26.05; HRMS (ESI) $\mathrm{m} / \mathrm{z}$ calculated for $\mathrm{C}_{17} \mathrm{H}_{20} \mathrm{~N}$ $\left[(\mathrm{M}+\mathrm{H})^{+}\right]$238.1590, found 238.1590; IR (film) 2925, 2851, 1596, 1547, 1448, 762, 697,

$616 \mathrm{~cm}^{-1}$. Spectra data are consistent with those reported in the literature: Org. Lett. 2018, 20, 3487-3490.

Compound 32b: ${ }^{1} \mathrm{H}$ NMR $\left(400 \mathrm{MHz}, \mathrm{CDCl}_{3}\right) \delta 7.62(\mathrm{~d}, J=7.1 \mathrm{~Hz}, 2 \mathrm{H}), 7.47(\mathrm{t}, J=7.3$ $\mathrm{Hz}, 2 \mathrm{H}), 7.41(\mathrm{t}, J=7.1 \mathrm{~Hz}, 1 \mathrm{H}), 7.18(\mathrm{~s}, 2 \mathrm{H}), 2.93-2.57(\mathrm{~m}, 2 \mathrm{H}), 2.07-1.95(\mathrm{~m}, 4 \mathrm{H})$, $1.91-1.82(\mathrm{~m}, 4 \mathrm{H}), 1.79-1.73(\mathrm{~m}, 2 \mathrm{H}), 1.60-1.39(\mathrm{~m}, 8 \mathrm{H}), 1.35-1.25(\mathrm{~m}, 2 \mathrm{H}) ;{ }^{13} \mathrm{C}$ NMR (101 MHz, $\left.\mathrm{CDCl}_{3}\right) \delta 166.10,149.09,139.57,128.89,128.54,127.13,116.05$, 46.58, 33.18, 26.59, 26.16; HRMS (ESI) $\mathrm{m} / \mathrm{z}$ calculated for $\mathrm{C}_{23} \mathrm{H}_{30} \mathrm{~N}\left[(\mathrm{M}+\mathrm{H})^{+}\right]$320.2373, found 320.2373; IR (film) 2925, 2850, 1596, 1552, 1448, 1408, 866, 762, $697 \mathrm{~cm}^{-1}$.

Spectra data are consistent with those reported in the literature: Org. Lett. 2018, 20, 34873490.

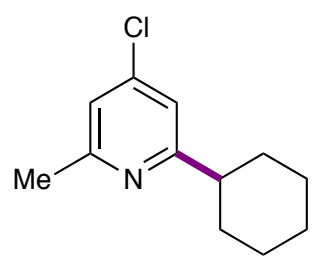

33

4-Chloro-2-cyclohexyl-6-methylpyridine (33): According to the general procedure (Conditions A), 4-chloro-2-methylpyridine ( $63.8 \mathrm{mg}, 0.50 \mathrm{mmol}, 1.0$ equiv.), Selectfluor (373.0 mg, $1.0 \mathrm{mmol}, 2.0$ equiv.), hydrochloric acid (43.0 $\mu 1,0.50 \mathrm{mmol}, 1.0$ equiv.), 
cyclohexane ( $0.55 \mathrm{ml}, 5.0 \mathrm{mmol}, 10.0$ equiv.), $5.0 \mathrm{~mL}$ of acetone were used. After 16 hours, the reaction mixture was subjected to the workup procedure outlined in the general procedure and purified by flash chromatography ( $10 \%$ ethyl acetate/hexanes) to provide the title compound as a colorless oil ( $59.8 \mathrm{mg}, 57 \%$ yield).

${ }^{1} \mathrm{H}$ NMR (400 MHz, $\left.\mathrm{CDCl}_{3}\right) \delta 6.96(\mathrm{~s}, 2 \mathrm{H}), 2.65(\mathrm{tt}, J=11.7,3.5 \mathrm{~Hz}, 1 \mathrm{H}), 2.49(\mathrm{~s}, 3 \mathrm{H})$, $1.97-1.88(\mathrm{~m}, 2 \mathrm{H}), 1.87-1.76(\mathrm{~m}, 2 \mathrm{H}), 1.76-1.68(\mathrm{~m}, 1 \mathrm{H}), 1.49-1.32(\mathrm{~m}, 4 \mathrm{H}), 1.31$ - $1.21(\mathrm{~m}, 1 \mathrm{H}) ;{ }^{13} \mathrm{C} \mathrm{NMR}\left(101 \mathrm{MHz}, \mathrm{CDCl}_{3}\right) \delta 167.62,158.92,144.24,120.72,117.91$, 46.47, 32.84, 26.41, 25.94, 24.32; HRMS (ESI) m/z calculated forC ${ }_{12} \mathrm{H}_{17} \mathrm{NCl}\left[(\mathrm{M}+\mathrm{H})^{+}\right]$ 210.1044, found 210.1044. IR (film) 2927, 2852, 1571, 1449, 1424, 1401, 868, 859, 848 $\mathrm{cm}^{-1}$.<smiles>CC(=O)c1cc(C)nc(C2CCCCC2)c1</smiles>
34

Methyl 2-cyclohexyl-6-methylisonicotinate (34): According to the general procedure (Conditions A), methyl 2-methylisonicotinate (75.6 mg, $0.50 \mathrm{mmol}, 1.0$ equiv.), Selectfluor (373.0 mg, $1.0 \mathrm{mmol}, 2.0$ equiv.), hydrochloric acid (43.0 $\mu 1,0.50 \mathrm{mmol}, 1.0$ equiv.), cyclohexane (1.10 ml, $10.0 \mathrm{mmol}, 20.0$ equiv.), $5.0 \mathrm{~mL}$ of acetone were used. After 16 hours, the reaction mixture was subjected to the workup procedure outlined in the general procedure and purified by flash chromatography (10\% ethyl acetate/hexanes) to provide the title compound as a colorless oil ( $85.2 \mathrm{mg}, 73 \%$ yield).

${ }^{1} \mathrm{H}$ NMR (400 MHz, $\left.\mathrm{CDCl}_{3}\right) \delta 7.45$ (s, 1H), $7.44(\mathrm{~s}, 1 \mathrm{H}), 3.86(\mathrm{~s}, 3 \mathrm{H}), 2.69$ (tt, $J=11.9$, $3.4 \mathrm{~Hz}, 1 \mathrm{H}), 2.52(\mathrm{~s}, 3 \mathrm{H}), 1.95-1.85(\mathrm{~m}, 2 \mathrm{H}), 1.84-1.74(\mathrm{~m}, 2 \mathrm{H}), 1.73-1.65(\mathrm{~m}, 1 \mathrm{H})$, $1.51-1.29(\mathrm{~m}, 4 \mathrm{H}), 1.28-1.18(\mathrm{~m}, 1 \mathrm{H}) ;{ }^{13} \mathrm{C} \mathrm{NMR}\left(101 \mathrm{MHz}, \mathrm{CDCl}_{3}\right) \delta 167.05,166.09$, 
$158.37,137.74,119.63,116.69,52.26,46.46,32.79,26.34,25.84,24.33$; HRMS (ESI) $\mathrm{m} / \mathrm{z}$ calculated for $\mathrm{C}_{14} \mathrm{H}_{20} \mathrm{O}_{2} \mathrm{~N}\left[(\mathrm{M}+\mathrm{H})^{+}\right]$234.1489, found 234.1488. IR (film) 2926, $2852,1732,1572,1438,1331,1232,1112,998,769 \mathrm{~cm}^{-1}$.

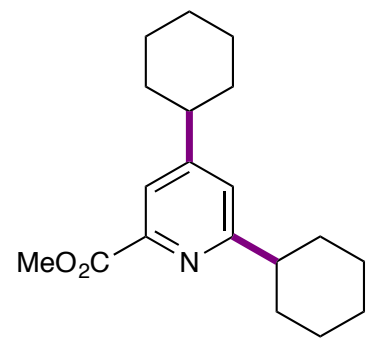

35

Methyl 4,6-dicyclohexylpicolinate (35): According to the general procedure (Conditions A), methyl picolinate $(68.6 \mathrm{mg}, 0.50 \mathrm{mmol}, 1.0$ equiv.), Selectfluor (466.3 mg, 1.25 mmol, 2.5 equiv.), hydrochloric acid (43.0 $\mu 1,0.50 \mathrm{mmol}, 1.0$ equiv.), cyclohexane (1.10 $\mathrm{ml}, 10.0 \mathrm{mmol}, 20.0$ equiv.), $10.0 \mathrm{~mL}$ of acetone were used. After 16 hours, the reaction mixture was subjected to the workup procedure outlined in the general procedure and purified by flash chromatography (10\% ethyl acetate/hexanes) to provide the title compound as a colorless oil $(86.7 \mathrm{mg}, 55 \%$ yield $)$.

${ }^{1} \mathrm{H}$ NMR (400 MHz, $\left.\mathrm{CDCl}_{3}\right) \delta 7.78(\mathrm{~d}, J=1.6 \mathrm{~Hz}, 1 \mathrm{H}), 7.15(\mathrm{~d}, J=1.6 \mathrm{~Hz}, 1 \mathrm{H}), 3.95(\mathrm{~s}$, $3 \mathrm{H}), 2.82(\mathrm{tt}, J=11.8,3.3 \mathrm{~Hz}, 1 \mathrm{H}), 2.53(\mathrm{tt}, J=11.8,3.1 \mathrm{~Hz}, 1 \mathrm{H}), 2.00-1.92(\mathrm{~m}, 2 \mathrm{H})$, $1.92-1.77(\mathrm{~m}, 6 \mathrm{H}), 1.77-1.69(\mathrm{~m}, 2 \mathrm{H}), 1.52-1.33(\mathrm{~m}, 8 \mathrm{H}), 1.30-1.19(\mathrm{~m}, 2 \mathrm{H}) ;{ }^{13} \mathrm{C}$ NMR (101 MHz, $\left.\mathrm{CDCl}_{3}\right) \delta 167.08,166.45,157.93,147.16,122.44,121.52,52.71,46.53$, 43.93, 33.42, 33.08, 26.41, 26.34, 25.94, 25.79; HRMS (ESI) $\mathrm{m} / \mathrm{z}$ calculated for $\mathrm{C}_{19} \mathrm{H}_{28} \mathrm{O}_{2} \mathrm{~N}\left[(\mathrm{M}+\mathrm{H})^{+}\right]$302.2115, found 302.2114; IR (film) 2926, 2851, 1745, 1720, $1602,1557,1447,1337,1240 \mathrm{~cm}^{-1}$. 


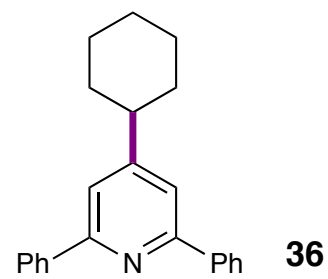

4-Cyclohexyl-2,6-diphenylpyridine (36): According to the general procedure (Conditions B), 2,6-diphenylpyridine (115.8 mg, $0.50 \mathrm{mmol}, 1.0$ equiv.), Selectfluor (466.3 mg, $1.25 \mathrm{mmol}, 2.5$ equiv.), trifluoroacetic acid (72.3 $\mu \mathrm{l}, 1.0 \mathrm{mmol}, 2.0$ equiv.), cyclohexane (1.10 ml, $10.0 \mathrm{mmol}, 20.0$ equiv.), $5.0 \mathrm{~mL}$ of acetonitrile were used. After 16 hours, the reaction mixture was subjected to the workup procedure outlined in the general procedure and purified by flash chromatography (5\% ethyl acetate/hexanes) to provide the title compound as a white solid (119.1 mg, 76\% yield).

${ }^{1} \mathrm{H}$ NMR $\left(400 \mathrm{MHz}, \mathrm{CDCl}_{3}\right) \delta 8.16(\mathrm{dd}, J=8.3,1.3 \mathrm{~Hz}, 4 \mathrm{H}), 7.56(\mathrm{~s}, 2 \mathrm{H}), 7.51(\mathrm{t}, J=7.4$ $\mathrm{Hz}, 4 \mathrm{H}), 7.43(\mathrm{t}, J=7.3 \mathrm{~Hz}, 2 \mathrm{H}), 2.66(\mathrm{tt}, J=11.8,3.4 \mathrm{~Hz}, 1 \mathrm{H}), 2.05-1.96(\mathrm{~m}, 2 \mathrm{H})$, $1.96-1.87(\mathrm{~m}, 2 \mathrm{H}), 1.87-1.78(\mathrm{~m}, 1 \mathrm{H}), 1.63-1.40(\mathrm{~m}, 4 \mathrm{H}), 1.40-1.29(\mathrm{~m}, 1 \mathrm{H}) ;{ }^{13} \mathrm{C}$ NMR (101 MHz, $\left.\mathrm{CDCl}_{3}\right) \delta 158.05,156.90,139.79,128.73,128.56,127.05,117.64$, 44.40, 33.68, 26.60, 25.98; HRMS (ESI) $\mathrm{m} / \mathrm{z}$ calculated for $\mathrm{C}_{23} \mathrm{H}_{24} \mathrm{~N}\left[(\mathrm{M}+\mathrm{H})^{+}\right]$314.1903, found 314.1902; IR (film) 2925, 2850, 1598, 1579, 1556, 1496, 1448, 1417, 862, 774, $738,694 \mathrm{~cm}^{-1}$. Spectra data are consistent with those reported in the literature: $R S C A d v$. 2017, 7, 13123-13129.<smiles>Cc1cc(Cl)nc(C2CCCCC2)n1</smiles>

4-Chloro-2-cyclohexyl-6-methylpyrimidine (37): According to the general procedure (Conditions A), 4-chloro-6-methylpyrimidine (64.3 $\mathrm{mg}, 0.50 \mathrm{mmol}, 1.0$ equiv.), 
Selectfluor (373.0 mg, $1.0 \mathrm{mmol}, 2.0$ equiv.), hydrochloric acid (43.0 $\mu 1,0.50 \mathrm{mmol}, 1.0$ equiv.), cyclohexane (1.10 ml, $10.0 \mathrm{mmol}, 20.0$ equiv.), $5.0 \mathrm{~mL}$ of acetone were used. After 16 hours, the reaction mixture was subjected to the workup procedure outlined in the general procedure and purified by flash chromatography ( $20 \%$ ethyl acetate/hexanes) to provide the title compound as a white solid ( $71.6 \mathrm{mg}, 68 \%$ yield).

${ }^{1} \mathrm{H} \mathrm{NMR}\left(400 \mathrm{MHz}, \mathrm{CDCl}_{3}\right) \delta 6.98(\mathrm{~s}, 1 \mathrm{H}), 2.78(\mathrm{tt}, J=11.9,3.5 \mathrm{~Hz}, 1 \mathrm{H}), 2.45(\mathrm{~s}, 3 \mathrm{H})$, $1.97-1.88(\mathrm{~m}, 2 \mathrm{H}), 1.84-1.77(\mathrm{~m}, 2 \mathrm{H}), 1.72-1.65(\mathrm{~m}, 1 \mathrm{H}), 1.64-1.53(\mathrm{~m}, 2 \mathrm{H}), 1.41$ $-1.23(\mathrm{~m}, 3 \mathrm{H}) ;{ }^{13} \mathrm{C} \mathrm{NMR}\left(101 \mathrm{MHz}, \mathrm{CDCl}_{3}\right) \delta 175.23,168.52,160.88,117.75,47.23$, 31.57, 26.01, 25.73, 23.86; HRMS (ESI) $\mathrm{m} / \mathrm{z}$ calculated for $\mathrm{C}_{11} \mathrm{H}_{16} \mathrm{~N}_{2} \mathrm{Cl}\left[(\mathrm{M}+\mathrm{H})^{+}\right]$ 211.0997, found 211.0997. IR (film) 2929, 2853, 1566, 1542, 1449, 1385, 1303, 877, 866 $\mathrm{cm}^{-1}$.

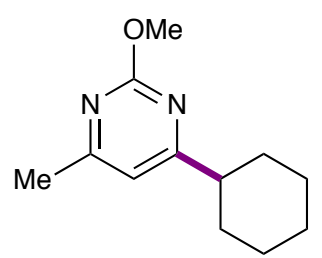

38

4-Cyclohexyl-2-methoxy-6-methylpyrimidine (38): According to the general procedure (Conditions B), 2-methoxy-4-methylpyrimidine (62.1 mg, $0.50 \mathrm{mmol}, 1.0$ equiv.), Selectfluor (410.3 mg, $1.1 \mathrm{mmol}, 2.2$ equiv.), trifluoroacetic acid (72.3 $\mu 1,1.0 \mathrm{mmol}, 2.0$ equiv.), cyclohexane (1.10 ml, $10.0 \mathrm{mmol}, 20.0$ equiv.), $5.0 \mathrm{~mL}$ of acetonitrile were used. After 16 hours, the reaction mixture was subjected to the workup procedure outlined in the general procedure and purified by flash chromatography ( $20 \%$ ethyl acetate/hexanes) to provide the title compound as a colorless oil ( $59.8 \mathrm{mg}, 58 \%$ yield).

${ }^{1} \mathrm{H} \mathrm{NMR}\left(400 \mathrm{MHz}, \mathrm{CDCl}_{3}\right) \delta 6.63(\mathrm{~s}, 1 \mathrm{H}), 3.97(\mathrm{~s}, 3 \mathrm{H}), 2.54(\mathrm{tt}, J=11.8,3.3 \mathrm{~Hz}, 1 \mathrm{H})$, $2.41(\mathrm{~s}, 3 \mathrm{H}), 1.94-1.87(\mathrm{~m}, 2 \mathrm{H}), 1.87-1.79(\mathrm{~m}, 2 \mathrm{H}), 1.76-1.69(\mathrm{~m}, 1 \mathrm{H}), 1.55-1.44$ 
(m, 2H), $1.43-1.31(\mathrm{~m}, 2 \mathrm{H}), 1.30-1.22(\mathrm{~m}, 1 \mathrm{H}) ;{ }^{13} \mathrm{C} \mathrm{NMR}\left(101 \mathrm{MHz}, \mathrm{CDCl}_{3}\right) \delta$

177.09, 169.16, 165.16, 111.57, 54.47, 45.66, 31.85, 26.20, 25.91, 23.91; HRMS (ESI)

$\mathrm{m} / \mathrm{z}$ calculated for $\mathrm{C}_{12} \mathrm{H}_{19} \mathrm{ON}_{2}\left[(\mathrm{M}+\mathrm{H})^{+}\right]$207.1492, found 207.1491. IR (film) 2927, $2853,1590,1556,1462,1355,1011,840,799 \mathrm{~cm}^{-1}$.<smiles>Cc1ncc(Br)c(C2CCCCC2)n1</smiles>

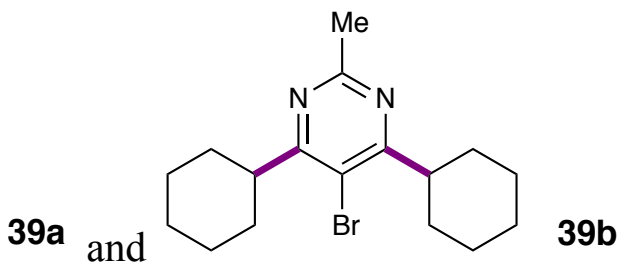

\section{5-Bromo-4-cyclohexyl-2-methylpyrimidine (39a) and 5-Bromo-4,6-dicyclohexyl-2-} methylpyrimidine (39b): According to the general procedure (Conditions A), 5-bromo2-methylpyrimidine ( $86.5 \mathrm{mg}, 0.50 \mathrm{mmol}, 1.0$ equiv.), Selectfluor $(373.0 \mathrm{mg}, 1.0 \mathrm{mmol}$, 2.0 equiv.), hydrochloric acid ( $43.0 \mu 1,0.50 \mathrm{mmol}, 1.0$ equiv.), cyclohexane ( $0.55 \mathrm{ml}, 5.0$ mmol, 10.0 equiv.), $5.0 \mathrm{~mL}$ of acetone were used. After 16 hours, the reaction mixture was subjected to the workup procedure outlined in the general procedure and purified by flash chromatography (5\% ethyl acetate/hexanes) to provide the title compounds as a colorless oil (51.0 mg, 40\% yield for 39a) and a white solid $(54.0 \mathrm{mg}, 32 \%$ yield for 39b).

Compound 39a: ${ }^{1} \mathrm{H}$ NMR (400 MHz, $\left.\mathrm{CDCl}_{3}\right) \delta 8.55(\mathrm{~s}, 1 \mathrm{H}), 3.04(\mathrm{tt}, J=11.5,3.3 \mathrm{~Hz}$, 1H), $2.63(\mathrm{~s}, 3 \mathrm{H}), 1.88-1.72(\mathrm{~m}, 5 \mathrm{H}), 1.64-1.53(\mathrm{~m}, 2 \mathrm{H}), 1.44-1.28(\mathrm{~m}, 3 \mathrm{H}) ;{ }^{13} \mathrm{C}$ NMR (101 MHz, $\left.\mathrm{CDCl}_{3}\right) \delta 171.53,166.39,158.00,117.75,43.79,30.51,26.10,25.72$, 25.40; HRMS (ESI) $\mathrm{m} / \mathrm{z}$ calculated for $\mathrm{C}_{11} \mathrm{H}_{16} \mathrm{~N}_{2} \mathrm{Br}\left[(\mathrm{M}+\mathrm{H})^{+}\right]$255.0491, found 255.0492; IR (film) 2927, 2853, 1556, 1526, 1449, 1422, 1049, 1017, $936 \mathrm{~cm}^{-1}$.

Compound 39b: ${ }^{1} \mathrm{H}$ NMR $\left(400 \mathrm{MHz}, \mathrm{CDCl}_{3}\right) \delta 3.15(\mathrm{tt}, J=11.6,3.3 \mathrm{~Hz}, 2 \mathrm{H}), 1.90-$ $1.70(\mathrm{~m}, 10 \mathrm{H}), 1.65-1.51(\mathrm{~m}, 4 \mathrm{H}), 1.65-1.51(\mathrm{~m}, 6 \mathrm{H}) ;{ }^{13} \mathrm{C} \mathrm{NMR}\left(101 \mathrm{MHz}, \mathrm{CDCl}_{3}\right) \delta$ 
$171.17,165.45,118.30,44.27,30.79,26.26,25.83,25.53 ; \mathrm{HRMS}$ (ESI) m/z calculated for $\mathrm{C}_{17} \mathrm{H}_{26} \mathrm{~N}_{2} \mathrm{Br}\left[(\mathrm{M}+\mathrm{H})^{+}\right]$337.1274, found 337.1272; IR (film) 2922, 2853, 1543, 1519, $1448,1395,1337,1013,936 \mathrm{~cm}^{-1}$.

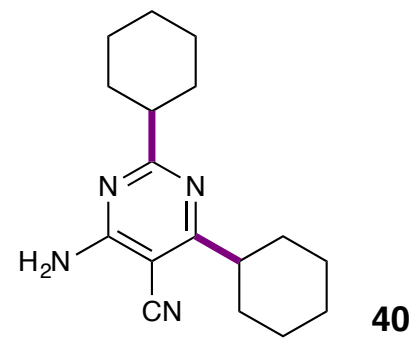

4-Amino-2,6-dicyclohexylpyrimidine-5-carbonitrile (40): According to the general procedure (Conditions A), 4-aminopyrimidine-5-carbonitrile (60.0 $\mathrm{mg}, 0.50 \mathrm{mmol}, 1.0$ equiv.), Selectfluor (466.3 mg, $1.25 \mathrm{mmol}, 2.5$ equiv.), hydrochloric acid (43.0 $\mu 1,0.50$ mmol, 1.0 equiv.), cyclohexane ( $1.10 \mathrm{ml}, 10.0 \mathrm{mmol}, 20.0$ equiv.), $5.0 \mathrm{~mL}$ of acetone were used. After 16 hours, the reaction mixture was subjected to the workup procedure outlined in the general procedure and purified by flash chromatography ( $20 \%$ ethyl acetate/hexanes) to provide the title compound as a white solid ( $42.7 \mathrm{mg}, 30 \%$ yield). ${ }^{1} \mathrm{H}$ NMR $\left(400 \mathrm{MHz}, \mathrm{CDCl}_{3}\right) \delta 5.56(\mathrm{~s}, 2 \mathrm{H}), 2.88(\mathrm{tt}, J=11.5,3.6 \mathrm{~Hz}, 1 \mathrm{H}), 2.62(\mathrm{tt}, J=$ 11.6, $3.5 \mathrm{~Hz}, 1 \mathrm{H}), 1.93-1.61(\mathrm{~m}, 12 \mathrm{H}), 1.61-1.47(\mathrm{~m}, 2 \mathrm{H}), 1.46-1.21(\mathrm{~m}, 6 \mathrm{H}) ;{ }^{13} \mathrm{C}$ NMR (101 MHz, $\left.\mathrm{CDCl}_{3}\right) \delta 177.70,176.33,163.55,115.57,85.72,47.54,45.07,31.28$, 30.89, 25.98, 25.91, 25.90, 25.59; HRMS (ESI) $\mathrm{m} / \mathrm{z}$ calculated for $\mathrm{C}_{17} \mathrm{H}_{25} \mathrm{~N}_{4}\left[(\mathrm{M}+\mathrm{H})^{+}\right]$ 285.2074, found 285.2072. IR (film) 3375, 3128, 2931, 2223, 1641, 1556, 1448, 993, $812 \mathrm{~cm}^{-1}$.<smiles>Nc1cc(Cl)nc(C2CCCCC2)n1</smiles> 
6-Chloro-2-cyclohexylpyrimidin-4-amine (41): According to the general procedure (Conditions A), 6-chloropyrimidine-4-amine (64.8 $\mathrm{mg}, 0.50 \mathrm{mmol}, 1.0$ equiv.), Selectfluor (373.0 mg, $1.0 \mathrm{mmol}, 2.0$ equiv.), hydrochloric acid (43.0 $\mu 1,0.50 \mathrm{mmol}, 1.0$ equiv.), cyclohexane (1.10 ml, $10.0 \mathrm{mmol}, 20.0$ equiv.), $5.0 \mathrm{~mL}$ of acetone were used. After 16 hours, the reaction mixture was subjected to the workup procedure outlined in the general procedure and purified by flash chromatography (50\% ethyl acetate/hexanes) to provide the title compound as a white solid (61.4 $\mathrm{mg}, 58 \%$ yield).

${ }^{1} \mathrm{H} \mathrm{NMR}\left(400 \mathrm{MHz}, \mathrm{CDCl}_{3}\right) \delta 6.29(\mathrm{~s}, 1 \mathrm{H}), 5.16(\mathrm{~s}, 2 \mathrm{H}), 2.62(\mathrm{tt}, J=11.8,3.5 \mathrm{~Hz}, 1 \mathrm{H})$, $1.96-1.86(\mathrm{~m}, 2 \mathrm{H}), 1.86-1.76(\mathrm{~m}, 2 \mathrm{H}), 1.74-1.66(\mathrm{~m}, 1 \mathrm{H}), 1.61-1.49(\mathrm{~m}, 2 \mathrm{H}), 1.38$ - $1.22(\mathrm{~m}, 3 \mathrm{H}) ;{ }^{13} \mathrm{C} \mathrm{NMR}\left(101 \mathrm{MHz}, \mathrm{CDCl}_{3}\right) \delta 174.89,163.48,160.14,100.94,47.05$, 31.39, 26.04, 25.79; HRMS (ESI) m/z calculated for $\mathrm{C}_{10} \mathrm{H}_{15} \mathrm{~N}_{3} \mathrm{Cl}\left[(\mathrm{M}+\mathrm{H})^{+}\right] 212.0949$, found 212.0949. IR (film) 3330, 3192, 2930, 1728, 1636, 1575, 1450, 1251, $896 \mathrm{~cm}^{-1}$.<smiles>Nc1nc(C2CCCCC2)cc(C(F)(F)F)n1</smiles>

42

4-Cyclohexyl-6-(trifluoromethyl)pyrimidin-2-amine (42): According to the general procedure (Conditions A), 4-(trifluoromethyl)pyrimidine-2-amine (81.6 mg, $0.50 \mathrm{mmol}$, 1.0 equiv.), Selectfluor (410.3 mg, $1.1 \mathrm{mmol}, 2.2$ equiv.), hydrochloric acid (43.0 $\mu 1,0.50$ mmol, 1.0 equiv.), cyclohexane (1.10 ml, $10.0 \mathrm{mmol}, 20.0$ equiv. $), 5.0 \mathrm{~mL}$ of acetone were used. After 16 hours, the reaction mixture was subjected to the workup procedure outlined in the general procedure and purified by flash chromatography ( $10 \%$ ethyl acetate/hexanes) to provide the title compound as a white solid (107.9 mg, 88\% yield). 
${ }^{1} \mathrm{H}$ NMR (400 MHz, $\left.\mathrm{CDCl}_{3}\right) \delta 6.78(\mathrm{~s}, 1 \mathrm{H}), 5.56(\mathrm{~s}, 2 \mathrm{H}), 2.54(\mathrm{tt}, J=11.8,3.4 \mathrm{~Hz}, 1 \mathrm{H})$, $1.95-1.80(\mathrm{~m}, 4 \mathrm{H}), 1.79-1.71(\mathrm{~m}, 1 \mathrm{H}), 1.51-1.21(\mathrm{~m}, 5 \mathrm{H}) ;{ }^{13} \mathrm{C} \mathrm{NMR}(101 \mathrm{MHz}$ $\left.\mathrm{CDCl}_{3}\right) \delta$ 179.04, 163.00, 156.43(q), 120.68(q), 104.19(q), 46.30, 31.71, 26.08, 25.77; HRMS (ESI) $\mathrm{m} / \mathrm{z}$ calculated for $\mathrm{C}_{11} \mathrm{H}_{15} \mathrm{~N}_{3} \mathrm{~F}_{3}\left[(\mathrm{M}+\mathrm{H})^{+}\right] 246.1213$, found 246.1211. IR (film) 3331, 3206, 2929, 1647, 1592, 1477, 1386, 1241, 1188, $832 \mathrm{~cm}^{-1}$.

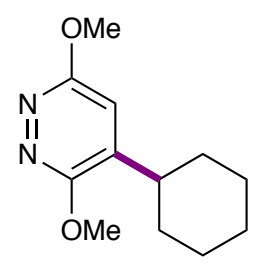

43

4-Cyclohexyl-3,6-dimethoxypyridazine (43): According to the general procedure (Conditions A), 3,6-dimethoxypyridazine (70.1 mg, $0.50 \mathrm{mmol}, 1.0$ equiv.), Selectfluor (373.0 mg, $1.0 \mathrm{mmol}, 2.0$ equiv.), hydrochloric acid (43.0 $\mu 1,0.50 \mathrm{mmol}, 1.0$ equiv.), cyclohexane (1.10 ml, $10.0 \mathrm{mmol}, 20.0$ equiv.), $5.0 \mathrm{~mL}$ of acetone were used. After 16 hours, the reaction mixture was subjected to the workup procedure outlined in the general procedure and purified by flash chromatography (10\% ethyl acetate/hexanes) to provide the title compound as a colorless oil ( $87.8 \mathrm{mg}, 79 \%$ yield).

${ }^{1} \mathrm{H}$ NMR (400 MHz, $\left.\mathrm{CDCl}_{3}\right) \delta 6.68(\mathrm{~s}, 1 \mathrm{H}), 4.02(\mathrm{~s}, 3 \mathrm{H}), 3.99$ (s, 3H), 2.67 (tt, $J=12.1$, $3.0 \mathrm{~Hz}, 1 \mathrm{H}), 1.90-1.77(\mathrm{~m}, 4 \mathrm{H}), 1.76-1.69(\mathrm{~m}, 1 \mathrm{H}), 1.45-1.30(\mathrm{~m}, 2 \mathrm{H}), 1.29-1.17$ (m, 3H); ${ }^{13} \mathrm{C}$ NMR $\left(101 \mathrm{MHz}, \mathrm{CDCl}_{3}\right) \delta 162.57,160.50,141.01,116.55,54.43,54.25$, $36.65,31.60,26.37,25.97$; HRMS (ESI) $\mathrm{m} / \mathrm{z}$ calculated for $\mathrm{C}_{12} \mathrm{H}_{19} \mathrm{O}_{2} \mathrm{~N}_{2}\left[(\mathrm{M}+\mathrm{H})^{+}\right]$ 223.1441, found 223.1441; IR (film) 2928, 2853, 1616, 1466, 1390, 1371, 1247, 1226, $1150,1015,771 \mathrm{~cm}^{-1}$. Spectra data are consistent with those reported in the literature: Angew. Chem. Int. Ed. 2015, 54, 4478-4481. 


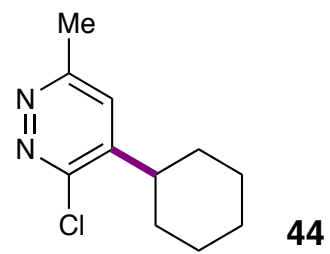

3-Chloro-4-cyclohexyl-6-methylpyridazine (44): According to the general procedure (Conditions A), 3-chloro-6-methylpyridazine (64.3 mg, $0.50 \mathrm{mmol}, 1.0$ equiv.), Selectfluor (373.0 mg, 1.0 mmol, 2.0 equiv.), hydrochloric acid (43.0 $\mu 1,0.50 \mathrm{mmol}, 1.0$ equiv.), cyclohexane (1.10 ml, $10.0 \mathrm{mmol}, 20.0$ equiv.), $5.0 \mathrm{~mL}$ of acetone were used. After 16 hours, the reaction mixture was subjected to the workup procedure outlined in the general procedure and purified by flash chromatography (20\% ethyl acetate/hexanes) to provide the title compound as a light yellow solid (88.5 $\mathrm{mg}, 84 \%$ yield).

${ }^{1} \mathrm{H}$ NMR (400 MHz, $\left.\mathrm{CDCl}_{3}\right) \delta 7.15(\mathrm{~s}, 1 \mathrm{H}), 2.84(\mathrm{tt}, J=11.8,2.9 \mathrm{~Hz}, 1 \mathrm{H}), 2.65(\mathrm{~s}, 3 \mathrm{H})$, $1.97-1.84(\mathrm{~m}, 4 \mathrm{H}), 1.83-1.75(\mathrm{~m}, 1 \mathrm{H}), 1.51-1.37(\mathrm{~m}, 2 \mathrm{H}), 1.36-1.21(\mathrm{~m}, 3 \mathrm{H}) ;{ }^{13} \mathrm{C}$ NMR $\left(101 \mathrm{MHz}, \mathrm{CDCl}_{3}\right) \delta 159.67,155.55,145.70,126.08,39.64,31.84,26.25,25.76$, 21.56; HRMS (ESI) m/z calculated for $\mathrm{C}_{11} \mathrm{H}_{16} \mathrm{~N}_{2} \mathrm{Cl}\left[(\mathrm{M}+\mathrm{H})^{+}\right]$211.0997, found 211.0996; IR (film) 2938, 2852, 1577, 1452, 1390, 1177, 1075, $950 \mathrm{~cm}^{-1}$. Spectra data are consistent with those reported in the literature: J. Heterocyclic Chem. 1991, 28, 583-587.

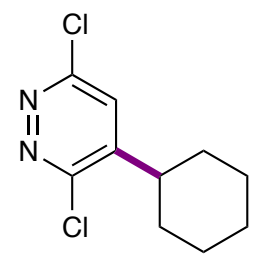

45

3,6-Dichloro-4-cyclohexylpyridazine (45): According to the general procedure (Conditions B), 3,6-dichloropyridazine (74.5 mg, $0.50 \mathrm{mmol}, 1.0$ equiv.), Selectfluor (410.3 mg, $1.1 \mathrm{mmol}, 2.2$ equiv.), trifluoroacetic acid (72.3 $\mu \mathrm{l}, 1.0 \mathrm{mmol}, 2.0$ equiv.), cyclohexane (1.10 ml, $10.0 \mathrm{mmol}, 20.0$ equiv.), $5.0 \mathrm{~mL}$ of acetonitrile were used. After 
16 hours, the reaction mixture was subjected to the workup procedure outlined in the general procedure and purified by flash chromatography ( $10 \%$ ethyl acetate/hexanes) to provide the title compound as a colorless oil ( $90.2 \mathrm{mg}, 78 \%$ yield).

${ }^{1} \mathrm{H}$ NMR (400 MHz, $\left.\mathrm{CDCl}_{3}\right) \delta 7.34$ (s, 1H), 2.85 (tt, $\left.J=11.9,3.0 \mathrm{~Hz}, 1 \mathrm{H}\right), 1.97-1.88$ (m, 4H), $1.83-1.78(\mathrm{~m}, 1 \mathrm{H}), 1.47-1.38(\mathrm{~m}, 2 \mathrm{H}), 1.35-1.24(\mathrm{~m}, 3 \mathrm{H}) ;{ }^{13} \mathrm{C}$ NMR $(101$ $\left.\mathrm{MHz}, \mathrm{CDCl}_{3}\right) \delta 156.63,156.23,148.68,127.05,40.07,31.69,26.11,25.60 ;$ HRMS (ESI) $\mathrm{m} / \mathrm{z}$ calculated for $\mathrm{C}_{10} \mathrm{H}_{13} \mathrm{~N}_{2} \mathrm{Cl}_{2}\left[(\mathrm{M}+\mathrm{H})^{+}\right]$231.0450, found 231.0450. IR (film) 2933, $2851,1557,1453,1344,1318,1129,932,560 \mathrm{~cm}^{-1}$. Spectra data are consistent with those reported in the literature: Chem. Eur. J. 2017, 23, 2537-2541.

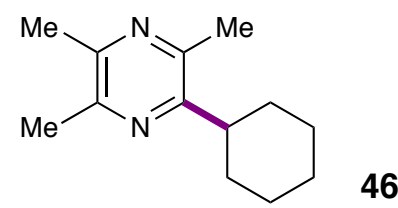

2-Cyclohexyl-3,5,6-trimethylpyrazine (46): According to the general procedure (Conditions A), 2,3,5-trimethylpyrazine (61.1 mg, $0.50 \mathrm{mmol}$, 1.0 equiv.), Selectfluor (373.0 mg, $1.0 \mathrm{mmol}, 2.0$ equiv.), hydrochloric acid (43.0 $\mu 1,0.50 \mathrm{mmol}, 1.0$ equiv.), cyclohexane ( $1.10 \mathrm{ml}, 10.0 \mathrm{mmol}, 20.0$ equiv.), $5.0 \mathrm{~mL}$ of acetone were used. After 16 hours, the reaction mixture was subjected to the workup procedure outlined in the general procedure and purified by flash chromatography ( $20 \%$ ethyl acetate/hexanes) to provide the title compound as a colorless oil ( $79.7 \mathrm{mg}, 78 \%$ yield).

${ }^{1} \mathrm{H}$ NMR (400 MHz, $\left.\mathrm{CDCl}_{3}\right) \delta 2.74(\mathrm{tt}, J=11.5,3.7 \mathrm{~Hz}, 1 \mathrm{H}), 2.47(\mathrm{~s}, 3 \mathrm{H}), 2.43(\mathrm{~s}, 3 \mathrm{H})$, $2.41(\mathrm{~s}, 3 \mathrm{H}), 1.88-1.78(\mathrm{~m}, 2 \mathrm{H}), 1.75-1.58(\mathrm{~m}, 5 \mathrm{H}), 1.42-1.25(\mathrm{~m}, 3 \mathrm{H}) ;{ }^{13} \mathrm{C}$ NMR $\left(101 \mathrm{MHz}, \mathrm{CDCl}_{3}\right) \delta 155.44,148.36,147.37,146.67,41.28,31.44,26.60,25.91,21.55$, 21.30, 20.71; HRMS (ESI) $\mathrm{m} / \mathrm{z}$ calculated for $\mathrm{C}_{13} \mathrm{H}_{21} \mathrm{~N}_{2}\left[(\mathrm{M}+\mathrm{H})^{+}\right]$205.1699, found 205.1699. IR (film) 2925, 2852, 1448, 1404, 1360, 1199, 1166, 1007, 979, $830 \mathrm{~cm}^{-1}$. 
Spectra data are consistent with those reported in the literature: Org. Lett. 2018, 20, 32293232.
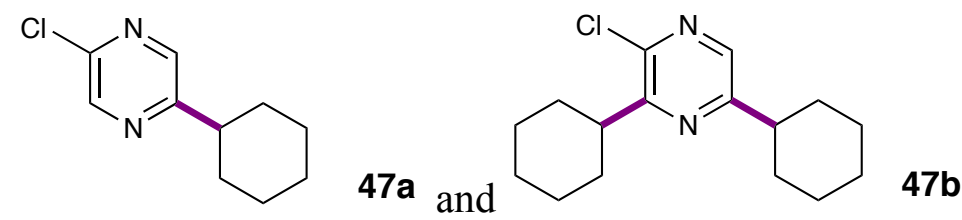

2-Chloro-5-cyclohexylpyrazine (47a) and 2-Chloro-3,5-dicyclohexylpyrazine (47b): According to the general procedure (Conditions A), 2-chloropyrazine (57.3 $\mathrm{mg}, 0.50$ mmol, 1.0 equiv.), Selectfluor (373.0 mg, $1.0 \mathrm{mmol}, 2.0$ equiv.), hydrochloric acid (43.0 $\mu \mathrm{l}, 0.50 \mathrm{mmol}, 1.0$ equiv.), cyclohexane (1.10 ml, $10.0 \mathrm{mmol}, 20.0$ equiv.), $5.0 \mathrm{~mL}$ of acetone were used. After 16 hours, the reaction mixture was subjected to the workup procedure outlined in the general procedure and purified by flash chromatography $(10 \%$ ethyl acetate/hexanes) to provide the title compounds as a colorless oil $(39.3 \mathrm{mg}, 40 \%$ yield for $\mathbf{4 7 a}$ ) and a colorless oil (15.3 $\mathrm{mg}, 11 \%$ yield for $\mathbf{4 7 b}$ ).

Compound 47a: ${ }^{1} \mathrm{H}$ NMR $\left(400 \mathrm{MHz}, \mathrm{CDCl}_{3}\right) \delta 8.41(\mathrm{~d}, J=2.5 \mathrm{~Hz}, 1 \mathrm{H}), 8.15(\mathrm{~d}, J=2.4$

$\mathrm{Hz}, 1 \mathrm{H}), 3.14(\mathrm{tt}, J=11.7,3.2 \mathrm{~Hz}, 1 \mathrm{H}), 1.92-1.80(\mathrm{~m}, 4 \mathrm{H}), 1.79-1.70(\mathrm{~m}, 1 \mathrm{H}), 1.64-$ $1.49(\mathrm{~m}, 2 \mathrm{H}), 1.48-1.35(\mathrm{~m}, 2 \mathrm{H}), 1.33-1.25(\mathrm{~m}, 1 \mathrm{H}) ;{ }^{13} \mathrm{C} \mathrm{NMR}\left(101 \mathrm{MHz}, \mathrm{CDCl}_{3}\right) \delta$ 159.81, 148.36, 142.12, 140.88, 77.32, 77.00, 76.68, 41.81, 30.96, 26.23, 25.81; HRMS (EI) $\mathrm{m} / \mathrm{z}$ calculated for C10H13CIN2 [M+] 196.0767, found 196.0770; IR (film) 2929, $2854,1449,1393,1151,1122,1081,1053,853 \mathrm{~cm}^{-1}$.

Compound 47b: ${ }^{1} \mathrm{H}$ NMR (400 MHz, $\left.\mathrm{CDCl}_{3}\right) \delta 7.99(\mathrm{~s}, 1 \mathrm{H}), 3.10(\mathrm{tt}, J=11.6,3.3 \mathrm{~Hz}$, $1 \mathrm{H}), 2.69(\mathrm{tt}, J=11.8,3.6 \mathrm{~Hz}, 1 \mathrm{H}), 1.95-1.80(\mathrm{~m}, 8 \mathrm{H}), 1.79-1.71(\mathrm{~m}, 2 \mathrm{H}), 1.66-1.49$ $(\mathrm{m}, 4 \mathrm{H}), 1.47-1.35(\mathrm{~m}, 4 \mathrm{H}), 1.34-1.24(\mathrm{~m}, 2 \mathrm{H}) ;{ }^{13} \mathrm{C} \mathrm{NMR}\left(101 \mathrm{MHz}, \mathrm{CDCl}_{3}\right) \delta$ $159.05,157.97,145.10,138.76,43.13,41.69,32.37,30.94,26.28,26.25,25.90,25.85$; 
HRMS (EI) m/z calculated for $\mathrm{C} 16 \mathrm{H} 23 \mathrm{CIN} 2\left[\mathrm{M}^{+}\right] 278.1550$, found 278.1555; IR (film) $2928,2853,1449,1419,1388,1345,1153,1120,1068,908,735 \mathrm{~cm}^{-1}$.

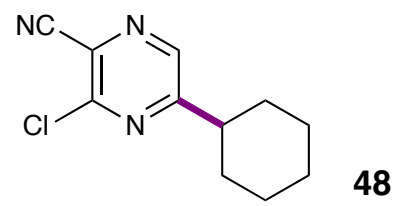

3-Chloro-5-cyclohexylpyrazine-2-carbonitrile (48): According to the general procedure (Conditions A), 3-chloropyrazine-2-carbonitrile (69.8 $\mathrm{mg}, 0.50 \mathrm{mmol}, 1.0$ equiv.), Selectfluor (410.3 mg, $1.1 \mathrm{mmol}, 2.2$ equiv.), hydrochloric acid (43.0 $\mu \mathrm{l}, 0.50$ mmol, 1.0 equiv.), cyclohexane ( $1.10 \mathrm{ml}, 10.0 \mathrm{mmol}, 20.0$ equiv.), $10.0 \mathrm{~mL}$ of acetone were used. After 16 hours, the reaction mixture was subjected to the workup procedure outlined in the general procedure and purified by flash chromatography (10\% ethyl acetate/hexanes) to provide the title compound as a colorless oil ( $78.7 \mathrm{mg}, 71 \%$ yield).

${ }^{1} \mathrm{H}$ NMR (400 MHz, $\left.\mathrm{CDCl}_{3}\right) \delta 8.48(\mathrm{~s}, 1 \mathrm{H}), 2.83(\mathrm{tt}, J=12.0,3.4 \mathrm{~Hz}, 1 \mathrm{H}), 1.98-1.86$ (m, 4H), $1.81-1.74(\mathrm{~m}, 1 \mathrm{H}), 1.62-1.53(\mathrm{~m}, 2 \mathrm{H}), 1.47-1.36(\mathrm{~m}, 2 \mathrm{H}), 1.35-1.27(\mathrm{~m}$, $1 \mathrm{H}) ;{ }^{13} \mathrm{C} \mathrm{NMR}\left(101 \mathrm{MHz}, \mathrm{CDCl}_{3}\right) \delta 165.49,150.77,141.77,127.25,114.06,44.06$, 31.87, 25.90, 25.43; HRMS (EI) $\mathrm{m} / \mathrm{z}$ calculated for $\mathrm{C}_{11} \mathrm{H}_{12} \mathrm{ClN}_{3}\left[\mathrm{M}^{+}\right] 221.0720$, found 221.0727; IR (film) 2932, 2855, 2239, 1549, 1507, 1450, 1330, 1297, 1156, 1078, 912, $734 \mathrm{~cm}^{-1}$.

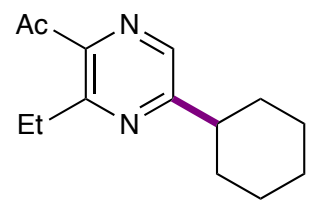

49

1-(5-Cyclohexyl-3-ethylpyrazin-2-yl)ethan-1-one (49): According to the general procedure (Conditions B), 1-(3-ethylpyrazin-2-yl)ethanone $(75.1 \mathrm{mg}, 0.50 \mathrm{mmol}, 1.0$ equiv.), Selectfluor (373.0 mg, $1.0 \mathrm{mmol}, 2.0$ equiv.), trifluoroacetic acid (72.3 $\mu \mathrm{l}, 1.0$ 
mmol, 2.0 equiv.), cyclohexane ( $1.10 \mathrm{ml}, 10.0 \mathrm{mmol}, 20.0$ equiv.), $5.0 \mathrm{~mL}$ of acetonitrile were used. After 16 hours, the reaction mixture was subjected to the workup procedure outlined in the general procedure and purified by flash chromatography $(10 \%$ ethyl acetate/hexanes) to provide the title compound as a colorless oil (98.7 mg, $85 \%$ yield).

${ }^{1} \mathrm{H} \mathrm{NMR}\left(400 \mathrm{MHz}, \mathrm{CDCl}_{3}\right) \delta 8.28(\mathrm{~s}, 1 \mathrm{H}), 3.11(\mathrm{q}, J=7.4 \mathrm{~Hz}, 2 \mathrm{H}), 2.75(\mathrm{tt}, J=12.0$, $3.5 \mathrm{~Hz}, 1 \mathrm{H}), 2.65(\mathrm{~s}, 3 \mathrm{H}), 1.96-1.88(\mathrm{~m}, 2 \mathrm{H}), 1.88-1.81(\mathrm{~m}, 2 \mathrm{H}), 1.77-1.71(\mathrm{~m}, 1 \mathrm{H})$, $1.62-1.50(\mathrm{~m}, 2 \mathrm{H}), 1.46-1.33(\mathrm{~m}, 2 \mathrm{H}), 1.33-1.26(\mathrm{~m}, 1 \mathrm{H}), 1.24(\mathrm{t}, J=7.5 \mathrm{~Hz}, 3 \mathrm{H})$; ${ }^{13} \mathrm{C}$ NMR $\left(101 \mathrm{MHz}, \mathrm{CDCl}_{3}\right) \delta 201.23,163.09,157.81,144.19,138.62,44.22,32.14$, 28.86, 28.02, 26.19, 25.76, 13.18; HRMS (ESI) $\mathrm{m} / \mathrm{z}$ calculated for $\mathrm{C}_{14} \mathrm{H}_{21} \mathrm{ON}_{2}\left[(\mathrm{M}+\mathrm{H})^{+}\right]$ 233.1648, found 233.1648. IR (film) 2930, 2853, 1698, 1552, 1450, 1353, 1280, 1157, $1076,954,608 \mathrm{~cm}^{-1}$.

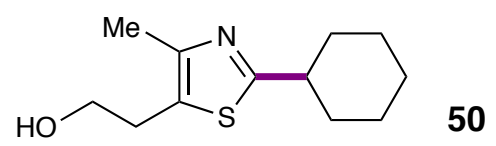

2-(2-Cyclohexyl-4-methylthiazol-5-yl)ethanol (50): According to the general procedure (Conditions A), 2-(4-methylthiazol-5-yl)ethanol (71.6 mg, $0.50 \mathrm{mmol}, 1.0$ equiv.), Selectfluor (466.3 mg, $1.25 \mathrm{mmol}, 2.5$ equiv.), hydrochloric acid (43.0 $\mu \mathrm{l}, 0.50 \mathrm{mmol}, 1.0$ equiv.), cyclohexane (1.10 ml, $10.0 \mathrm{mmol}, 20.0$ equiv.), $10.0 \mathrm{~mL}$ of acetone were used. After 16 hours, the reaction mixture was subjected to the workup procedure outlined in the general procedure and purified by flash chromatography (50\% ethyl acetate/hexanes) to provide the title compound as a light yellow oil ( $42.8 \mathrm{mg}, 38 \%$ yield).

${ }^{1} \mathrm{H} \mathrm{NMR}\left(400 \mathrm{MHz}, \mathrm{CDCl}_{3}\right) \delta 3.78(\mathrm{t}, J=6.5 \mathrm{~Hz}, 2 \mathrm{H}), 2.92(\mathrm{t}, J=6.5 \mathrm{~Hz}, 2 \mathrm{H}), 2.87(\mathrm{tt}, J$ $=8.0,4.1 \mathrm{~Hz}, 1 \mathrm{H}), 2.56(\mathrm{~s}, 1 \mathrm{H}), 2.30(\mathrm{~s}, 3 \mathrm{H}), 2.11-2.02(\mathrm{~m}, 2 \mathrm{H}), 1.85-1.75(\mathrm{~m}, 2 \mathrm{H})$, $1.75-1.66(\mathrm{~m}, 1 \mathrm{H}), 1.50-1.30(\mathrm{~m}, 4 \mathrm{H}), 1.29-1.18(\mathrm{~m}, 1 \mathrm{H}) ;{ }^{13} \mathrm{C} \mathrm{NMR}(101 \mathrm{MHz}$, 
$\left.\mathrm{CDCl}_{3}\right) \delta 173.96,147.61,125.82,62.87,42.52,33.76,29.78,26.06,25.72,14.76 ; \mathrm{HRMS}$ (ESI) $\mathrm{m} / \mathrm{z}$ calculated for $\mathrm{C}_{12} \mathrm{H}_{20} \mathrm{ONS}\left[(\mathrm{M}+\mathrm{H})^{+}\right] 226.1260$, found 226.1260. IR (film) $3284,2927,2852,1448,1379,1196,1054,891 \mathrm{~cm}^{-1}$.

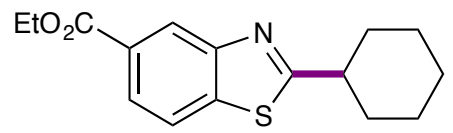

51

Ethyl 2-cyclohexylbenzo $[d]$ thiazole-5-carboxylate (51): According to the general procedure (Conditions B), ethyl benzo[d]thiazole-5-carboxylate $(103.6 \mathrm{mg}, 0.50 \mathrm{mmol}$, 1.0 equiv.), Selectfluor (373.0 mg, $1.0 \mathrm{mmol}, 2.0$ equiv.), trifluoroacetic acid (72.3 $\mu 1,1.0$ mmol, 2.0 equiv.), cyclohexane ( $1.10 \mathrm{ml}, 10.0 \mathrm{mmol}, 20.0$ equiv.), $5.0 \mathrm{~mL}$ of acetonitrile were used. After 16 hours, the reaction mixture was subjected to the workup procedure outlined in the general procedure and purified by flash chromatography $(20 \%$ ethyl acetate/hexanes) to provide the title compound as a colorless oil (123.0 mg, 85\% yield). ${ }^{1} \mathrm{H}$ NMR $\left(400 \mathrm{MHz}, \mathrm{CDCl}_{3}\right) \delta 8.64(\mathrm{~d}, J=1.6 \mathrm{~Hz}, 1 \mathrm{H}), 8.00(\mathrm{dd}, J=8.3,1.6 \mathrm{~Hz}, 1 \mathrm{H})$, $7.85(\mathrm{~d}, J=8.4 \mathrm{~Hz}, 1 \mathrm{H}), 4.39$ (q, $J=7.1 \mathrm{~Hz}, 2 \mathrm{H}), 3.08$ (tt, $J=11.5,3.6 \mathrm{~Hz}, 1 \mathrm{H}), 2.23-$ $2.13(\mathrm{~m}, 2 \mathrm{H}), 1.92-1.82(\mathrm{~m}, 2 \mathrm{H}), 1.79-1.70(\mathrm{~m}, 1 \mathrm{H}), 1.69-1.56(\mathrm{~m}, 2 \mathrm{H}), 1.50-1.41$ $(\mathrm{m}, 2 \mathrm{H}), 1.39(\mathrm{t}, J=7.1 \mathrm{~Hz}, 3 \mathrm{H}), 1.35-1.25(\mathrm{~m}, 1 \mathrm{H}) ;{ }^{13} \mathrm{C} \mathrm{NMR}\left(101 \mathrm{MHz}, \mathrm{CDCl}_{3}\right) \delta$ $178.76,166.38,152.86,139.27,128.39,125.09,123.96,121.27,61.02,43.32,33.18$, 25.89, 25.65, 14.22; HRMS (ESI) $\mathrm{m} / \mathrm{z}$ calculated for $\mathrm{C}_{16} \mathrm{H}_{20} \mathrm{O}_{2} \mathrm{NS}\left[(\mathrm{M}+\mathrm{H})^{+}\right]$290.1209, found 290.1209; IR (film) 2927, 2851, 1712, 1602, 1367, 1255, 1297, 1211, 1145, 1088, $764 \mathrm{~cm}^{-1}$.

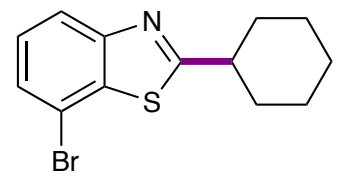


7-Bromo-2-cyclohexylbenzo[d]thiazole (52): According to the general procedure (Conditions B), 7-bromobenzo[d]thiazole (107.0 mg, $0.50 \mathrm{mmol}, 1.0$ equiv.), Selectfluor (373.0 mg, $1.0 \mathrm{mmol}, 2.0$ equiv.), trifluoroacetic acid (72.3 $\mu 1,1.0 \mathrm{mmol}, 2.0$ equiv.), cyclohexane (1.10 ml, $10.0 \mathrm{mmol}, 20.0$ equiv.), $5.0 \mathrm{~mL}$ of acetonitrile were used. After 16 hours, the reaction mixture was subjected to the workup procedure outlined in the general procedure and purified by flash chromatography ( $10 \%$ ethyl acetate/hexanes) to provide the title compound as a white solid ( $121.4 \mathrm{mg}, 82 \%$ yield).

${ }^{1} \mathrm{H}$ NMR $\left(400 \mathrm{MHz}, \mathrm{CDCl}_{3}\right) \delta 7.88(\mathrm{~d}, J=8.1 \mathrm{~Hz}, 1 \mathrm{H}), 7.45(\mathrm{~d}, J=7.8 \mathrm{~Hz}, 1 \mathrm{H}), 7.30(\mathrm{t}$, $J=7.9 \mathrm{~Hz}, 1 \mathrm{H}), 3.07(\mathrm{tt}, J=11.7,3.6 \mathrm{~Hz}, 1 \mathrm{H}), 2.24-2.13(\mathrm{~m}, 2 \mathrm{H}), 1.93-1.82(\mathrm{~m}, 2 \mathrm{H})$, $1.80-1.70(\mathrm{~m}, 1 \mathrm{H}), 1.69-1.55(\mathrm{~m}, 2 \mathrm{H}), 1.49-1.36(\mathrm{~m}, 2 \mathrm{H}), 1.36-1.25(\mathrm{~m}, 1 \mathrm{H}) ;{ }^{13} \mathrm{C}$ NMR (101 MHz, $\left.\mathrm{CDCl}_{3}\right) \delta 177.82,152.83,137.36,127.25,126.91,121.30,113.92$, 43.42, 33.29, 25.92, 25.65; HRMS (ESI) $\mathrm{m} / \mathrm{z}$ calculated for $\mathrm{C}_{13} \mathrm{H}_{15} \mathrm{NBrS}\left[(\mathrm{M}+\mathrm{H})^{+}\right]$ 296.0103, found 296.0104; IR (film) 2936, 2849, 1692, 1592, 1447, 1360, 1233, 912, $886,605 \mathrm{~cm}^{-1}$.

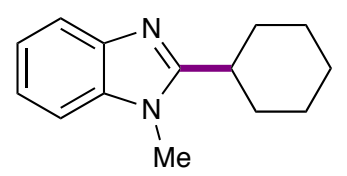

53

2-Cyclohexyl-1-methyl-1H-benzo $[\boldsymbol{d}]$ imidazole (53): According to the general procedure (Conditions A), 1-methyl-1H-benzo[d]imidazole $(66.1 \mathrm{mg}, 0.50 \mathrm{mmol}, 1.0$ equiv.), Selectfluor (560.0 mg, $1.5 \mathrm{mmol}, 3.0$ equiv.), hydrochloric acid (43.0 $\mu \mathrm{l}, 0.50$ mmol, 1.0 equiv.), cyclohexane ( $1.65 \mathrm{ml}, 15.0 \mathrm{mmol}, 30.0$ equiv.), $10.0 \mathrm{~mL}$ of acetone were used. After 16 hours, the reaction mixture was subjected to the workup procedure outlined in the general procedure and purified by flash chromatography (50\% ethyl acetate/hexanes) to provide the title compound as a white solid (27.8 mg, $26 \%$ yield). 
${ }^{1} \mathrm{H}$ NMR $\left(400 \mathrm{MHz}, \mathrm{CDCl}_{3}\right) \delta 7.74-7.61(\mathrm{~m}, 1 \mathrm{H}), 7.30-7.19(\mathrm{~m}, 1 \mathrm{H}), 7.19-7.12(\mathrm{~m}$, 2H), $3.66(\mathrm{~s}, 3 \mathrm{H}), 2.77$ (tt, $J=11.7,3.5 \mathrm{~Hz}, 1 \mathrm{H}), 1.99-1.88(\mathrm{~m}, 2 \mathrm{H}), 1.88-1.80(\mathrm{~m}$, $2 \mathrm{H}), 1.80-1.64(\mathrm{~m}, 3 \mathrm{H}), 1.43-1.23(\mathrm{~m}, 3 \mathrm{H}) ;{ }^{13} \mathrm{C} \mathrm{NMR}\left(101 \mathrm{MHz}, \mathrm{CDCl}_{3}\right) \delta 158.90$, $142.18,135.46,121.91,121.71,119.11,108.82,36.24,31.37,29.52,26.25,25.73$;

HRMS (ESI) $\mathrm{m} / \mathrm{z}$ calculated for $\mathrm{C}_{14} \mathrm{H}_{18} \mathrm{~N}_{2}\left[(\mathrm{M}+\mathrm{H})^{+}\right] 215.1543$, found 215.1541; IR (film) $2930,2851,1615,1505,1464,1447,1410,1330,1279,1008,743 \mathrm{~cm}^{-1}$. Spectra data are consistent with those reported in the literature: Org. Lett. 2017, 19, 6594-6597.

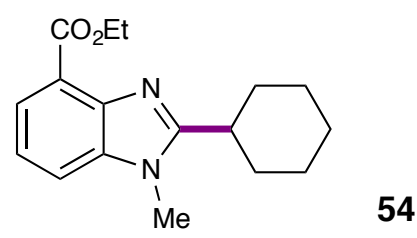

Ethyl 2-cyclohexyl-1-methyl-1H-benzo[d] imidazole-4-carboxylate (54): According to the general procedure (Conditions A), ethyl 1-methyl-1H-benzo[ $d]$ imidazole-4carboxylate (102.1 mg, $0.50 \mathrm{mmol}, 1.0$ equiv.), Selectfluor (373.0 mg, $1.0 \mathrm{mmol}, 2.0$ equiv.), hydrochloric acid (43.0 $\mu \mathrm{l}, 0.50 \mathrm{mmol}, 1.0$ equiv.), cyclohexane (1.10 ml, 10.0 mmol, 20.0 equiv.), $10.0 \mathrm{~mL}$ of acetone were used. After 16 hours, the reaction mixture was subjected to the workup procedure outlined in the general procedure and purified by flash chromatography (50\% ethyl acetate/hexanes) to provide the title compound as a light yellow solid (51.1 mg, 50\% yield).

${ }^{1} \mathrm{H}$ NMR $\left(400 \mathrm{MHz}, \mathrm{CDCl}_{3}\right) \delta 7.88(\mathrm{~d}, J=7.6 \mathrm{~Hz}, 1 \mathrm{H}), 7.44(\mathrm{~d}, J=8.0 \mathrm{~Hz}, 1 \mathrm{H}), 7.23$ (t, $J=7.8 \mathrm{~Hz}, 1 \mathrm{H}), 4.46(\mathrm{q}, J=7.1 \mathrm{~Hz}, 2 \mathrm{H}), 3.75(\mathrm{~s}, 3 \mathrm{H}), 2.89(\mathrm{tt}, J=11.4,3.8 \mathrm{~Hz}, 1 \mathrm{H})$, $2.06-1.97(\mathrm{~m}, 2 \mathrm{H}), 1.96-1.84(\mathrm{~m}, 4 \mathrm{H}), 1.79-1.72(\mathrm{~m}, 1 \mathrm{H}), 1.44(\mathrm{t}, J=7.1 \mathrm{~Hz}, 3 \mathrm{H})$, $1.42-1.32(\mathrm{~m}, 3 \mathrm{H}) ;{ }^{13} \mathrm{C} \mathrm{NMR}\left(101 \mathrm{MHz}, \mathrm{CDCl}_{3}\right) \delta 166.12,160.65,141.86,136.98$, $124.33,120.92,120.92,113.09,60.68,36.68,30.80,29.74,26.16,25.63,14.29$; HRMS 
(ESI) $\mathrm{m} / \mathrm{z}$ calculated for $\mathrm{C}_{17} \mathrm{H}_{23} \mathrm{O}_{2} \mathrm{~N}_{2}\left[(\mathrm{M}+\mathrm{H})^{+}\right]$287.1754, found 287.1754; IR (film) 2930, 2853, 1721, 1457, 1424, 1296, 1249, 1207, 1108, $751 \mathrm{~cm}^{-1}$.

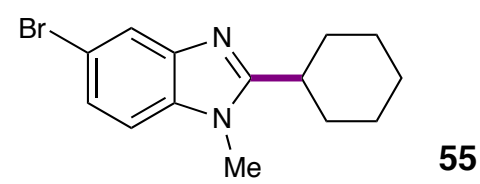

5-Bromo-2-cyclohexyl-1-methyl-1H-benzoimidazole (55): According to the general procedure (Conditions A), 5-bromo-1-methyl-benzoimidazole (105.5 mg, $0.50 \mathrm{mmol}, 1.0$ equiv.), Selectfluor (466.3 mg, $1.25 \mathrm{mmol}, 2.5$ equiv.), hydrochloric acid (43.0 $\mu 1,0.50$ mmol, 1.0 equiv. $)$, cyclohexane (1.10 ml, $10.0 \mathrm{mmol}, 20.0$ equiv. $), 5.0 \mathrm{~mL}$ of acetone were used. After 16 hours, the reaction mixture was subjected to the workup procedure outlined in the general procedure and purified by flash chromatography $(50 \%$ ethyl acetate/hexanes) to provide the title compound as a white solid (61.6 mg, $42 \%$ yield).

${ }^{1} \mathrm{H}$ NMR $\left(400 \mathrm{MHz}, \mathrm{CDCl}_{3}\right) \delta 7.85(\mathrm{~d}, J=1.5 \mathrm{~Hz}, 1 \mathrm{H}), 7.32(\mathrm{dd}, J=8.5,1.7 \mathrm{~Hz}, 1 \mathrm{H})$, $7.14(\mathrm{~d}, J=8.5 \mathrm{~Hz}, 1 \mathrm{H}), 3.71(\mathrm{~s}, 3 \mathrm{H}), 2.82(\mathrm{tt}, J=11.9,3.5 \mathrm{~Hz}, 1 \mathrm{H}), 2.03-1.87(\mathrm{~m}, 4 \mathrm{H})$, $1.83-1.71(\mathrm{~m}, 3 \mathrm{H}), 1.50-1.31(\mathrm{~m}, 3 \mathrm{H}) ;{ }^{13} \mathrm{C} \mathrm{NMR}\left(101 \mathrm{MHz}, \mathrm{CDCl}_{3}\right) \delta 160.07,143.65$ 134.53, 124.87, 122.01, 114.68, 110.05, 36.32, 31.31, 29.69, 26.19, 25.70; HRMS (ESI) $\mathrm{m} / \mathrm{z}$ calculated for $\mathrm{C}_{14} \mathrm{H}_{18} \mathrm{~N}_{2} \mathrm{Br}\left[(\mathrm{M}+\mathrm{H})^{+}\right]$293.0648, found 293.0648; IR (film) 2923, $2849,1505,1470,1424,1324,1289,1043,907,867,794 \mathrm{~cm}^{-1}$.

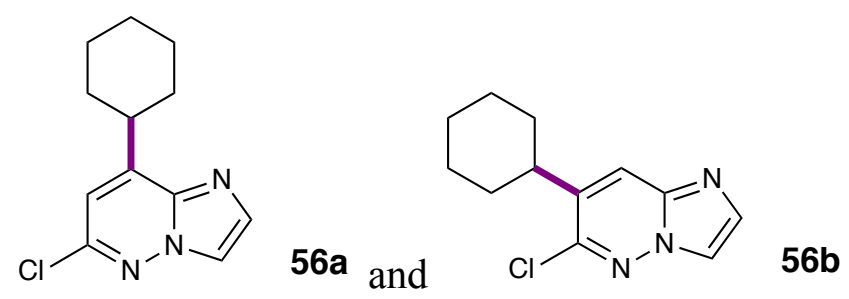

6-Chloro-8-cyclohexylimidazo[1,2-b]pyridazine (56a) and 6-Chloro-7cyclohexylimidazo[1,2-b]pyridazine (56b): According to the general procedure (Conditions A), 6-chloroimidazo[1,2-b]pyridazine (76.8 mg, $0.50 \mathrm{mmol}, 1.0$ equiv.), 
Selectfluor (373.0 mg, $1.0 \mathrm{mmol}, 2.0$ equiv.), hydrochloric acid (43.0 $\mu 1,0.50 \mathrm{mmol}, 1.0$ equiv.), cyclohexane (1.10 ml, $10.0 \mathrm{mmol}, 20.0$ equiv.), $5.0 \mathrm{~mL}$ of acetone were used. After 16 hours, the reaction mixture was subjected to the workup procedure outlined in the general procedure and purified by flash chromatography ( $20 \%$ ethyl acetate/hexanes) to provide the title compounds as a white solid (73.1 $\mathrm{mg}, 62 \%$ yield for $\mathbf{5 6 a}$ ) and a white solid (21.2 mg, 18\% yield for $\mathbf{5 6 b})$.

Compound 56a: ${ }^{1} \mathrm{H}$ NMR $\left(400 \mathrm{MHz}, \mathrm{CDCl}_{3}\right) \delta 7.81(\mathrm{~d}, J=1.2 \mathrm{~Hz}, 1 \mathrm{H}), 7.64(\mathrm{~d}, J=1.2$ $\mathrm{Hz}, 1 \mathrm{H}), 6.78$ (s, 1H), 3.27 (tt, $J=11.2,3.3 \mathrm{~Hz}, 1 \mathrm{H}), 2.06-1.93(\mathrm{~m}, 2 \mathrm{H}), 1.88-1.77$ (m, 2H), $1.76-1.68(\mathrm{~m}, 1 \mathrm{H}), 1.50-1.36(\mathrm{~m}, 4 \mathrm{H}), 1.31-1.16(\mathrm{~m}, 1 \mathrm{H}) ;{ }^{13} \mathrm{C} \mathrm{NMR}(101 \mathrm{MHz}$, $\left.\mathrm{CDCl}_{3}\right) \delta 147.97,147.27,137.79,132.99,117.10,114.53,38.66,31.94,26.15,25.86$; HRMS (ESI) m/z calculated for $\mathrm{C}_{12} \mathrm{H}_{15} \mathrm{~N}_{3} \mathrm{Cl}\left[(\mathrm{M}+\mathrm{H})^{+}\right] 236.0949$, found 236.0949; IR (film) $3086,2926,2852,1541,1473,1278,1144,1120,926,841,799 \mathrm{~cm}^{-1}$. Spectra data are consistent with those reported in the literature: Angew. Chem. Int. Ed. 2017, 56, 12336-12339.

Compound 56b: ${ }^{1} \mathrm{H}$ NMR (400 MHz, $\left.\mathrm{CDCl}_{3}\right) \delta 7.86(\mathrm{~s}, 1 \mathrm{H}), 7.79(\mathrm{~s}, 1 \mathrm{H}), 7.71(\mathrm{~s}, 1 \mathrm{H})$, $2.86(\mathrm{tt}, J=11.5,3.1 \mathrm{~Hz}, 1 \mathrm{H}), 2.06-1.95(\mathrm{~m}, 2 \mathrm{H}), 1.95-1.85(\mathrm{~m}, 2 \mathrm{H}), 1.84-1.76(\mathrm{~m}$, 1H), $1.53-1.22(\mathrm{~m}, 5 \mathrm{H}) ;{ }^{13} \mathrm{C} \mathrm{NMR}\left(101 \mathrm{MHz}, \mathrm{CDCl}_{3}\right) \delta 148.33,138.90,136.76,133.78$, $122.63,115.98,40.08,32.80,26.48,25.80 ; \mathrm{HRMS}$ (ESI) m/z calculated for $\mathrm{C}_{12} \mathrm{H}_{15} \mathrm{~N}_{3} \mathrm{Cl}$ $\left[(\mathrm{M}+\mathrm{H})^{+}\right]$236.0949, found 236.0949; IR (film) 3142, 2929, 2848, 1448, 1326, 1287, $1120,1063,721,690 \mathrm{~cm}^{-1}$.<smiles>CCOc1cn2nc(Cl)cc(C3CCCCC3)c2n1</smiles> 
Ethyl 6-chloro-8-cyclohexylimidazo[1,2-b]pyridazine-2-carboxylate (57): According to the general procedure (Conditions A), ethyl 6-chloroimidazo[1,2-b]pyridazine-2carboxylate (112.8 mg, $0.50 \mathrm{mmol}, 1.0$ equiv.), Selectfluor (373.0 mg, $1.0 \mathrm{mmol}, 2.0$ equiv.), hydrochloric acid (43.0 $\mu \mathrm{l}, 0.50 \mathrm{mmol}, 1.0$ equiv.), cyclohexane (1.10 ml, 10.0 mmol, 20.0 equiv.), $5.0 \mathrm{~mL}$ of acetone were used. After 16 hours, the reaction mixture was subjected to the workup procedure outlined in the general procedure and purified by flash chromatography (10\% ethyl acetate/hexanes) to provide the title compound as a colorless oil (120.0 mg, 78\% yield).

${ }^{1} \mathrm{H}$ NMR (400 MHz, $\left.\mathrm{CDCl}_{3}\right) \delta 8.38(\mathrm{~s}, 1 \mathrm{H}), 6.91(\mathrm{~s}, 1 \mathrm{H}), 4.45(\mathrm{q}, J=7.1 \mathrm{~Hz}, 2 \mathrm{H}), 3.52$

$(\mathrm{tt}, J=11.6,3.4 \mathrm{~Hz}, 1 \mathrm{H}), 2.10-2.01(\mathrm{~m}, 2 \mathrm{H}), 1.91-1.77(\mathrm{~m}, 3 \mathrm{H}), 1.59-1.43(\mathrm{~m}, 4 \mathrm{H})$,

$1.42(\mathrm{t}, J=7.1 \mathrm{~Hz}, 3 \mathrm{H}), 1.33-1.23(\mathrm{~m}, 1 \mathrm{H}) ;{ }^{13} \mathrm{C} \mathrm{NMR}\left(101 \mathrm{MHz}, \mathrm{CDCl}_{3}\right) \delta 162.65$, $149.90,149.16,138.39,135.89,121.24,116.48,61.39,37.70,32.26,25.97,25.84,14.34$;

HRMS (ESI) $\mathrm{m} / \mathrm{z}$ calculated for $\mathrm{C}_{15} \mathrm{H}_{19} \mathrm{O}_{2} \mathrm{~N}_{3} \mathrm{Cl}\left[(\mathrm{M}+\mathrm{H})^{+}\right]$308.1160, found 308.1160; IR (film) 2933, 2854, 1732, 1590, 1544, 1451, 1339, 1279, 1207, 1130, $732 \mathrm{~cm}^{-1}$.

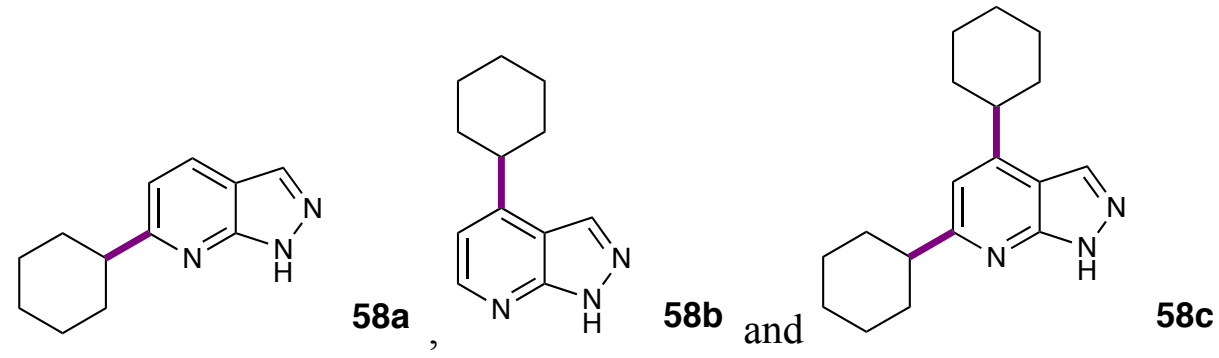

\section{6-Cyclohexyl-1H-pyrazolo[3,4-b]pyridine}

(58a), 4-Cyclohexyl-1H-pyrazolo[3,4b]pyridine (58b) and 4,6-Dicyclohexyl-1H-pyrazolo[3,4-b]pyridine (58c): According to the general procedure (Conditions A), 1H-pyrazolo[3,4-b]pyridine (59.6 $\mathrm{mg}, 0.50$ mmol, 1.0 equiv.), Selectfluor (466.3 mg, $1.25 \mathrm{mmol}, 2.5$ equiv.), hydrochloric acid (43.0 $\mu \mathrm{l}, 0.50 \mathrm{mmol}, 1.0$ equiv.), cyclohexane (1.10 ml, $10.0 \mathrm{mmol}, 20.0$ equiv.), $5.0 \mathrm{~mL}$ of 
acetone were used. After 16 hours, the reaction mixture was subjected to the workup procedure outlined in the general procedure and purified by flash chromatography $(50 \%$ ethyl acetate/hexanes) to provide the title compounds as a white solid (34.2 mg, 34\% yield for 58a), a white solid (18.1 mg, 18\% yield for 58b) and a white solid (35.4 mg, $25 \%$ yield for $\mathbf{5 8 c}$ ).

Compound 58a: ${ }^{1} \mathrm{H}$ NMR $\left(400 \mathrm{MHz}, \mathrm{CDCl}_{3}\right) \delta 12.99(\mathrm{~s}, 1 \mathrm{H}), 8.06(\mathrm{~s}, 1 \mathrm{H}), 8.05(\mathrm{~d}, J=$ $8.4 \mathrm{~Hz}, 1 \mathrm{H}), 7.08(\mathrm{~d}, J=8.3 \mathrm{~Hz}, 1 \mathrm{H}), 2.94(\mathrm{tt}, J=12.0,3.4 \mathrm{~Hz}, 1 \mathrm{H}), 2.13-1.98(\mathrm{~m}, 2 \mathrm{H})$, $1.98-1.88(\mathrm{~m}, 2 \mathrm{H}), 1.84-1.68(\mathrm{~m}, 3 \mathrm{H}), 1.56-1.38(\mathrm{~m}, 3 \mathrm{H}) ;{ }^{13} \mathrm{C}$ NMR $(101 \mathrm{MHz}$, $\left.\mathrm{CDCl}_{3}\right) \delta 166.60,151.65,133.55,130.64,115.75,113.68,47.02,33.08,26.61,25.95 ;$

HRMS (ESI) m/z calculated for $\mathrm{C}_{12} \mathrm{H}_{16} \mathrm{~N}_{3}\left[(\mathrm{M}+\mathrm{H})^{+}\right]$202.1339, found 202.1338; IR (film) $2917,2849,1607,1584,1447,1127,938,859,754 \mathrm{~cm}^{-1}$.

Compound 58b: ${ }^{1} \mathrm{H}$ NMR (400 MHz, $\left.\mathrm{CDCl}_{3}\right) \delta 12.59(\mathrm{~s}, 1 \mathrm{H}), 8.55(\mathrm{~d}, J=4.9 \mathrm{~Hz}, 1 \mathrm{H})$, $8.20(\mathrm{~s}, 1 \mathrm{H}), 7.01(\mathrm{~d}, J=4.9 \mathrm{~Hz}, 1 \mathrm{H}), 3.00(\mathrm{tt}, J=12.0,3.4 \mathrm{~Hz}, 1 \mathrm{H}), 2.07-1.97(\mathrm{~m}, 2 \mathrm{H})$ $1.97-1.87(\mathrm{~m}, 2 \mathrm{H}), 1.87-1.79(\mathrm{~m}, 1 \mathrm{H}), 1.70-1.56(\mathrm{~m}, 2 \mathrm{H}), 1.56-1.42(\mathrm{~m}, 2 \mathrm{H}), 1.40$ $-1.28(\mathrm{~m}, 1 \mathrm{H}) ;{ }^{13} \mathrm{C} \mathrm{NMR}\left(101 \mathrm{MHz}, \mathrm{CDCl}_{3}\right) \delta 152.70,151.86,148.69,132.56,114.84$, 113.86, 42.53, 33.22, 26.56, 26.03; HRMS (ESI) m/z calculated for $\mathrm{C}_{12} \mathrm{H}_{16} \mathrm{~N}_{3}\left[(\mathrm{M}+\mathrm{H})^{+}\right]$ 202.1339, found 202.1339; IR (film) 2984, 2933, 1740, 1448, 1374, 1243, 1047, 918, 734 $\mathrm{cm}^{-1}$.

Compound 58c: ${ }^{1} \mathrm{H}$ NMR $\left(400 \mathrm{MHz}, \mathrm{CDCl}_{3}\right) \delta 8.05(\mathrm{~s}, 1 \mathrm{H}), 6.82(\mathrm{~s}, 1 \mathrm{H}), 2.88(\mathrm{tt}, J=$ $11.9,3.4 \mathrm{~Hz}, 1 \mathrm{H}), 2.82(\mathrm{tt}, J=11.9,3.4 \mathrm{~Hz}, 1 \mathrm{H}), 2.05-1.90(\mathrm{~m}, 4 \mathrm{H}), 1.90-1.80(\mathrm{~m}$, 4H), $1.80-1.63(\mathrm{~m}, 4 \mathrm{H}), 1.63-1.51(\mathrm{~m}, 2 \mathrm{H}), 1.47-1.24(\mathrm{~m}, 6 \mathrm{H}) ;{ }^{13} \mathrm{C} \mathrm{NMR}(101 \mathrm{MHz}$, $\left.\mathrm{CDCl}_{3}\right) \delta 166.67,152.61,151.89,132.47,113.24,112.66,47.06,42.88,33.26,33.11$, 26.65, 26.61, 26.10, 25.96; HRMS (ESI) $\mathrm{m} / \mathrm{z}$ calculated for $\mathrm{C}_{18} \mathrm{H}_{26} \mathrm{~N}_{3}\left[(\mathrm{M}+\mathrm{H})^{+}\right]$ 
284.2121, found 284.2121; IR (film) 2921, 2853, 1606, 1584, 1446, 1324, 1165, 937, $865,845 \mathrm{~cm}^{-1}$.<smiles>Clc1nc(C2CCCCC2)c2nc(C3CCCCC3)[nH]c2n1</smiles>

2-Chloro-6,8-dicyclohexyl-9H-purine (59): According to the general procedure (Conditions A), 2-chloro-9H-purine (77.3 mg, $0.50 \mathrm{mmol}, 1.0$ equiv.), Selectfluor (466.3 mg, $1.25 \mathrm{mmol}, 2.5$ equiv.), hydrochloric acid (43.0 $\mu 1,0.50 \mathrm{mmol}, 1.0$ equiv.), cyclohexane (1.10 ml, $10.0 \mathrm{mmol}, 20.0$ equiv.), $5.0 \mathrm{~mL}$ of acetone were used. After 16 hours, the reaction mixture was subjected to the workup procedure outlined in the general procedure and purified by flash chromatography ( $50 \%$ ethyl acetate/hexanes) to provide the title compound as a white solid ( $27.2 \mathrm{mg}, 23 \%$ yield).

${ }^{1} \mathrm{H}$ NMR (400 MHz, $\left.\mathrm{CDCl}_{3}\right) \delta 12.28(\mathrm{~s}, 1 \mathrm{H}), 3.50-3.35(\mathrm{~m}, 1 \mathrm{H}), 3.10(\mathrm{tt}, J=11.9,3.6$

$\mathrm{Hz}, 1 \mathrm{H}), 2.21-2.10(\mathrm{~m}, 2 \mathrm{H}), 1.98-1.68(\mathrm{~m}, 12 \mathrm{H}), 1.53-1.31(\mathrm{~m}, 6 \mathrm{H}) ;{ }^{13} \mathrm{C}$ NMR $(101$ $\left.\mathrm{MHz}, \mathrm{CDCl}_{3}\right) \delta 167.33,161.64,154.47,152.10,130.73,41.43,38.90,31.32,30.97$, 25.96, 25.82, 25.68, 25.45; HRMS (ESI) $\mathrm{m} / \mathrm{z}$ calculated for $\mathrm{C}_{17} \mathrm{H}_{24} \mathrm{~N}_{4} \mathrm{Cl}\left[(\mathrm{M}+\mathrm{H})^{+}\right]$ 319.1684, found 319.1684. IR (film) 3063, 2928, 2852, 1615, 1560, 1515, 1449, 1427, $1375,1266,1167,997,931 \mathrm{~cm}^{-1}$.<smiles>Cn1c(C2CCCCC2)nc2c(Cl)nc(Cl)nc21</smiles>
60

2,6-Dichloro-8-cyclohexyl-9-methyl-9H-purine (60): According to the general procedure (Conditions A), 2,6-dichloro-9-methyl-9H-purine $(101.5 \mathrm{mg}, 0.50 \mathrm{mmol}, 1.0$ 
equiv.), Selectfluor ( $466.3 \mathrm{mg}, 1.25 \mathrm{mmol}, 2.5$ equiv.), hydrochloric acid (43.0 $\mu 1,0.50$ mmol, 1.0 equiv.), cyclohexane ( $1.10 \mathrm{ml}, 10.0 \mathrm{mmol}, 20.0$ equiv.), $5.0 \mathrm{~mL}$ of acetone were used. After 16 hours, the reaction mixture was subjected to the workup procedure outlined in the general procedure and purified by flash chromatography $(50 \%$ ethyl acetate/hexanes) to provide the title compound as a white solid (106.9 $\mathrm{mg}, 75 \%$ yield).

${ }^{1} \mathrm{H}$ NMR (400 MHz, $\left.\mathrm{CDCl}_{3}\right) \delta 3.81(\mathrm{~s}, 3 \mathrm{H}), 2.88(\mathrm{tt}, J=11.8,3.5 \mathrm{~Hz}, 1 \mathrm{H}), 2.02-1.88$ (m, 4H), $1.88-1.75(\mathrm{~m}, 3 \mathrm{H}), 1.49-1.31(\mathrm{~m}, 3 \mathrm{H}) ;{ }^{13} \mathrm{C}$ NMR $\left(101 \mathrm{MHz}, \mathrm{CDCl}_{3}\right) \delta$ 163.08, 154.64, 151.61, 149.47, 130.14, 36.85, 30.72, 29.20, 25.89, 25.42; HRMS (ESI) $\mathrm{m} / \mathrm{z}$ calculated for $\mathrm{C}_{12} \mathrm{H}_{15} \mathrm{Cl}_{2} \mathrm{~N}_{4}\left[(\mathrm{M}+\mathrm{H})^{+}\right]$285.0668, found 285.0668. IR (film) 2931, $2855,1589,1560,1474,1362,1281,1153,974,893,830 \mathrm{~cm}^{-1}$.

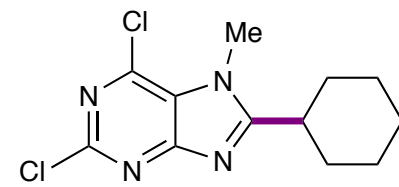

61

2,6-Dichloro-8-cyclohexyl-7-methyl-7H-purine (61): According to the general procedure (Conditions A), 2,6-dichloro-7-methyl-7H-purine (101.5 mg, $0.50 \mathrm{mmol}, 1.0$ equiv.), Selectfluor (373.0 mg, $1.0 \mathrm{mmol}, 2.0$ equiv.), hydrochloric acid (43.0 $\mu 1,0.50$ mmol, 1.0 equiv.), cyclohexane (1.10 ml, $10.0 \mathrm{mmol}, 20.0$ equiv.), $5.0 \mathrm{~mL}$ of acetone were used. After 16 hours, the reaction mixture was subjected to the workup procedure outlined in the general procedure and purified by flash chromatography $(50 \%$ ethyl acetate/hexanes) to provide the title compound as a white solid (111.2 $\mathrm{mg}, 78 \%$ yield).

${ }^{1} \mathrm{H}$ NMR $\left(400 \mathrm{MHz}, \mathrm{CDCl}_{3}\right) \delta 4.05(\mathrm{~s}, 3 \mathrm{H}), 2.88(\mathrm{tt}, J=11.6,3.4 \mathrm{~Hz}, 1 \mathrm{H}), 1.98-1.86$ $(\mathrm{m}, 4 \mathrm{H}), 1.85-1.71(\mathrm{~m}, 3 \mathrm{H}), 1.52-1.26(\mathrm{~m}, 3 \mathrm{H}) ;{ }^{13} \mathrm{C} \mathrm{NMR}\left(101 \mathrm{MHz}, \mathrm{CDCl}_{3}\right) \delta$ 167.53, 162.81, 152.31, 142.01, 122.68, 36.45, 32.06, 30.76, 25.74, 25.39; HRMS (ESI) 
$\mathrm{m} / \mathrm{z}$ calculated for $\mathrm{C}_{12} \mathrm{H}_{15} \mathrm{~N}_{4} \mathrm{Cl}_{2}\left[(\mathrm{M}+\mathrm{H})^{+}\right]$285.0668, found 285.0668; IR (film) 2931, $2854,1595,1537,1482,1445,1398,1260,1215,1027,870 \mathrm{~cm}^{-1}$.<smiles>O=[R20]C1CCCC(c2cc(Oc3ccc(F)cc3)c3c(Cl)cc(Cl)cc3n2)C1</smiles>

5,7-Dichloro-2-cyclohexyl-4-(4-fluorophenoxy)quinoline (62): According to the general procedure (Conditions B), 5,7-dichloro-4-(4-fluorophenoxy)quinoline (154.1 mg, $0.50 \mathrm{mmol}, 1.0$ equiv.), Selectfluor (373.0 mg, $1.0 \mathrm{mmol}, 2.0$ equiv.), trifluoroacetic acid (72.3 $\mu \mathrm{l}, 1.0 \mathrm{mmol}, 2.0$ equiv.), cyclohexane ( $1.10 \mathrm{ml}, 10.0 \mathrm{mmol}, 20.0$ equiv.), $5.0 \mathrm{~mL}$ of acetonitrile were used. After 16 hours, the reaction mixture was subjected to the workup procedure outlined in the general procedure and purified by flash chromatography ( $10 \%$ ethyl acetate/hexanes) to provide the title compound as a colorless oil (169.9 mg, 87\% yield).

${ }^{1} \mathrm{H}$ NMR $\left(400 \mathrm{MHz}, \mathrm{CDCl}_{3}\right) \delta 7.94(\mathrm{~d}, J=2.0 \mathrm{~Hz}, 1 \mathrm{H}), 7.46(\mathrm{~d}, J=2.1 \mathrm{~Hz}, 1 \mathrm{H}), 7.19-$ $7.04(\mathrm{~m}, 4 \mathrm{H}), 6.53(\mathrm{~s}, 1 \mathrm{H}), 2.69(\mathrm{tt}, J=11.8,3.4 \mathrm{~Hz}, 1 \mathrm{H}), 1.91-1.69(\mathrm{~m}, 5 \mathrm{H}), 1.48-$ $1.21(\mathrm{~m}, 5 \mathrm{H}) ;{ }^{13} \mathrm{C} \mathrm{NMR}\left(101 \mathrm{MHz}, \mathrm{CDCl}_{3}\right) \delta 169.42,162.23,159.74(\mathrm{~d}), 151.25,150.22$ (d), 134.63, 129.79, 128.47, 127.38, 121.85 (d), 116.91 (d), 116.88, 105.63, 47.10, 32.25, 26.21, 25.77; HRMS (ESI) $\mathrm{m} / \mathrm{z}$ calculated for $\mathrm{C}_{21} \mathrm{H}_{19} \mathrm{ONCl}_{2} \mathrm{~F}\left[(\mathrm{M}+\mathrm{H})^{+}\right] 390.0822$, found 390.0822; IR (film) 2928, 2852, 1738, 1600, 1551, 1503, 1489, 1365, 1240, 1196, 853 $\mathrm{cm}^{-1}$. Spectra data are consistent with those reported in the literature: Asian J. Org. Chem. 2018, 7, 1307-1310. 


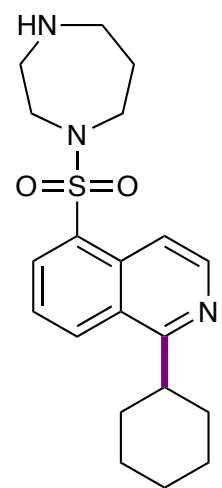

63

5-((1,4-Diazepan-1-yl)sulfonyl)-1-cyclohexylisoquinoline (63): According to the general procedure (Conditions B), Fasudil (163.9 mg, $0.50 \mathrm{mmol}, 1.0$ equiv.), Selectfluor (466.3 mg, $1.25 \mathrm{mmol}, 2.5$ equiv.), trifluoroacetic acid (72.3 $\mu 1,1.0 \mathrm{mmol}, 2.0$ equiv.), cyclohexane (1.10 ml, $10.0 \mathrm{mmol}, 20.0$ equiv.), $5.0 \mathrm{~mL}$ of acetonitrile were used. After 16 hours, the reaction mixture was subjected to the workup procedure outlined in the general procedure and purified by flash chromatography ( $20 \%$ methanol/ethyl acetate) to provide the title compound as a light yellow oil (95.2 $\mathrm{mg}, 51 \%$ yield).

${ }^{1} \mathrm{H}$ NMR $\left(400 \mathrm{MHz}, \mathrm{CDCl}_{3}\right) \delta 8.60(\mathrm{~d}, J=6.1 \mathrm{~Hz}, 1 \mathrm{H}), 8.46(\mathrm{~d}, J=8.6 \mathrm{~Hz}, 1 \mathrm{H}), 8.29(\mathrm{~d}$, $J=7.4 \mathrm{~Hz}, 1 \mathrm{H}), 8.25(\mathrm{~d}, J=6.1 \mathrm{~Hz}, 1 \mathrm{H}), 7.63(\mathrm{dd}, J=8.6,7.4 \mathrm{~Hz}, 1 \mathrm{H}), 3.55(\mathrm{tt}, J=11.4$, $3.4 \mathrm{~Hz}, 1 \mathrm{H}), 3.49$ (t, $J=6.1 \mathrm{~Hz}, 2 \mathrm{H}), 3.44(\mathrm{t}, J=5.2 \mathrm{~Hz}, 2 \mathrm{H}), 2.98(\mathrm{t}, J=5.4 \mathrm{~Hz}, 2 \mathrm{H})$, $2.95(\mathrm{t}, J=5.8 \mathrm{~Hz}, 2 \mathrm{H}), 2.63(\mathrm{br} \mathrm{s}, 2 \mathrm{H}), 2.03-1.89(\mathrm{~m}, 4 \mathrm{H}), 1.90-1.72(\mathrm{~m}, 5 \mathrm{H}), 1.59-$ $1.44(\mathrm{~m}, 2 \mathrm{H}), 1.44-1.33(\mathrm{~m}, 1 \mathrm{H}) ;{ }^{13} \mathrm{C} \mathrm{NMR}\left(101 \mathrm{MHz}, \mathrm{CDCl}_{3}\right) \delta$ 166.57, 143.77, $135.03,132.23,132.20,130.26,126.86,125.10,115.41,50.68,50.07,47.43,47.34$, 41.99, 32.66, 30.88, 26.71, 26.06; HRMS (ESI) $\mathrm{m} / \mathrm{z}$ calculated for $\mathrm{C}_{20} \mathrm{H}_{28} \mathrm{~N}_{3} \mathrm{O}_{2} \mathrm{~S}$ $\left[(\mathrm{M}+\mathrm{H})^{+}\right]$374.1897, found 374.1897. IR (film) 2927, 2825, 1609, 1552, 1450, 1325, $1150,820,734,594 \mathrm{~cm}^{-1}$. Spectra data are consistent with those reported in the literature: J. Am. Chem. Soc. 2017, 139, 12251-12258. 


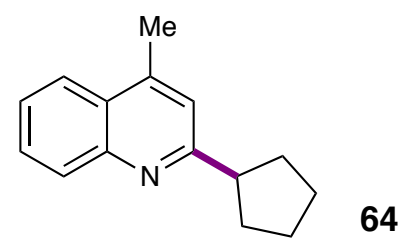

2-Cyclopentyl-4-methylquinoline (64): According to the general procedure (Conditions A), lepidine (71.6 mg, $0.50 \mathrm{mmol}, 1.0$ equiv.), Selectfluor (373.0 mg, $1.0 \mathrm{mmol}, 2.0$ equiv.), hydrochloric acid (43.0 $\mu \mathrm{l}, 0.50 \mathrm{mmol}, 1.0$ equiv.), cyclopentane $(0.90 \mathrm{ml}, 10.0$ mmol, 20.0 equiv.), $5.0 \mathrm{~mL}$ of acetone were used. After 16 hours, the reaction mixture was subjected to the workup procedure outlined in the general procedure and purified by flash chromatography (10\% ethyl acetate/hexanes) to provide the title compound as a colorless oil ( $70.8 \mathrm{mg}, 67 \%$ yield).

${ }^{1} \mathrm{H}$ NMR (400 MHz, $\left.\mathrm{CDCl}_{3}\right) \delta 8.05(\mathrm{~d}, J=8.4 \mathrm{~Hz}, 1 \mathrm{H}), 7.93(\mathrm{~d}, J=8.3 \mathrm{~Hz}, 1 \mathrm{H}), 7.66$ (ddd, $J=8.4,6.8,1.4 \mathrm{~Hz}, 1 \mathrm{H}), 7.49$ (ddd, $J=8.2,6.8,1.3 \mathrm{~Hz}, 1 \mathrm{H}), 7.18(\mathrm{~s}, 1 \mathrm{H}), 3.34(\mathrm{p}$, $J=8.4 \mathrm{~Hz}, 1 \mathrm{H}), 2.67(\mathrm{~s}, 3 \mathrm{H}), 2.24-2.10(\mathrm{~m}, 2 \mathrm{H}), 1.95-1.81(\mathrm{~m}, 4 \mathrm{H}), 1.81-1.68(\mathrm{~m}$, $2 \mathrm{H}) ;{ }^{13} \mathrm{C}$ NMR $\left(101 \mathrm{MHz}, \mathrm{CDCl}_{3}\right) \delta 165.86,147.39,144.16,129.36,128.91,126.92$, 125.33, 123.49, 120.59, 48.71, 33.54, 26.01, 18.78; HRMS (ESI) m/z calculated for $\mathrm{C}_{15} \mathrm{H}_{18} \mathrm{~N}\left[(\mathrm{M}+\mathrm{H})^{+}\right]$212.1434, found 212.1433; IR (film) 2951, 2866, 1603, 1559, 1507, $1448,1411,1345,758 \mathrm{~cm}^{-1}$. Spectra data are consistent with those reported in the literature: Org. Lett. 2017, 19, 6594-6597.

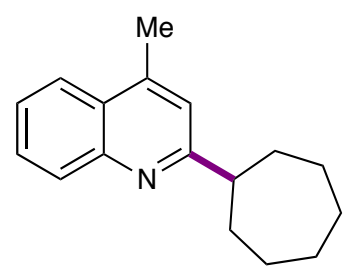

65

2-Cycloheptyl-4-methylquinoline (65): According to the general procedure (Conditions A), lepidine (71.6 mg, $0.50 \mathrm{mmol}, 1.0$ equiv.), Selectfluor (373.0 mg, $1.0 \mathrm{mmol}, 2.0$ 
equiv.), hydrochloric acid (43.0 $\mu \mathrm{l}, 0.50 \mathrm{mmol}, 1.0$ equiv.), cycloheptane (1.20 ml, 10.0 mmol, 20.0 equiv.), $5.0 \mathrm{~mL}$ of acetone were used. After 16 hours, the reaction mixture was subjected to the workup procedure outlined in the general procedure and purified by flash chromatography (10\% ethyl acetate/hexanes) to provide the title compound as a colorless oil (105.3 mg, $88 \%$ yield).

${ }^{1} \mathrm{H}$ NMR $\left(400 \mathrm{MHz}, \mathrm{CDCl}_{3}\right) \delta 8.05(\mathrm{~d}, J=8.4 \mathrm{~Hz}, 1 \mathrm{H}), 7.90(\mathrm{~d}, J=8.3 \mathrm{~Hz}, 1 \mathrm{H}), 7.64$ (ddd, $J=8.4,6.9,1.5 \mathrm{~Hz}, 1 \mathrm{H}), 7.46$ (ddd, $J=8.2,6.8,1.3 \mathrm{~Hz}, 1 \mathrm{H}), 7.12(\mathrm{~s}, 1 \mathrm{H}), 3.03(\mathrm{tt}$, $J=10.6,3.5 \mathrm{~Hz}, 1 \mathrm{H}), 2.65$ (s, 3H), $2.09-1.97$ (m, 2H), $1.91-1.77$ (m, 4H), $1.77-1.69$ $(\mathrm{m}, 2 \mathrm{H}), 1.69-1.58(\mathrm{~m}, 4 \mathrm{H}) ;{ }^{13} \mathrm{C} \mathrm{NMR}\left(101 \mathrm{MHz}, \mathrm{CDCl}_{3}\right) \delta$ 167.97, 147.24, 144.28, $129.29,128.82,126.82,125.21,123.41,120.17,49.45,34.96,27.87,27.36,18.72$; HRMS (ESI) m/z calculated for $\mathrm{C}_{17} \mathrm{H}_{22} \mathrm{~N}\left[(\mathrm{M}+\mathrm{H})^{+}\right] 240.1747$, found 240.1746; IR (film) $2922,2853,1603,1560,1507,1447,1411,1341,757 \mathrm{~cm}^{-1}$.

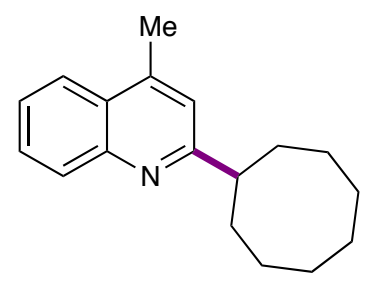

66

2-Cyclooctyl-4-methylquinoline (66): According to the general procedure (Conditions A), lepidine (71.6 mg, $0.50 \mathrm{mmol}, 1.0$ equiv.), Selectfluor (373.0 mg, $1.0 \mathrm{mmol}, 2.0$ equiv.), hydrochloric acid (43.0 $\mu 1,0.50 \mathrm{mmol}, 1.0$ equiv.), cyclooctane (1.30 ml, 10.0 mmol, 20.0 equiv.), $5.0 \mathrm{~mL}$ of acetone were used. After 16 hours, the reaction mixture was subjected to the workup procedure outlined in the general procedure and purified by flash chromatography (10\% ethyl acetate/hexanes) to provide the title compound as a colorless oil ( $96.3 \mathrm{mg}, 76 \%$ yield). 
${ }^{1} \mathrm{H}$ NMR (400 MHz, $\left.\mathrm{CDCl}_{3}\right) \delta 8.06(\mathrm{~d}, J=8.4 \mathrm{~Hz}, 1 \mathrm{H}), 7.92(\mathrm{~d}, J=8.3 \mathrm{~Hz}, 1 \mathrm{H}), 7.65$ (ddd, $J=8.4,6.8,1.4 \mathrm{~Hz}, 1 \mathrm{H}), 7.47$ (ddd, $J=8.2,6.8,1.3 \mathrm{~Hz}, 1 \mathrm{H}), 7.12(\mathrm{~s}, 1 \mathrm{H}), 3.12$ (tt, $J=9.8,3.6 \mathrm{~Hz}, 1 \mathrm{H}), 2.66(\mathrm{~d}, J=0.8 \mathrm{~Hz}, 3 \mathrm{H}), 2.03-1.94(\mathrm{~m}, 2 \mathrm{H}), 1.94-1.78(\mathrm{~m}, 4 \mathrm{H})$, $1.78-1.57(\mathrm{~m}, 8 \mathrm{H}) ;{ }^{13} \mathrm{C}$ NMR $\left(101 \mathrm{MHz}, \mathrm{CDCl}_{3}\right) \delta 168.72,147.26,144.26,129.36$, $128.85,126.81,125.25,123.44,120.59,47.44,33.44,26.55,26.36,26.09,18.76$; HRMS (ESI) $\mathrm{m} / \mathrm{z}$ calculated for $\mathrm{C}_{18} \mathrm{H}_{24} \mathrm{~N}\left[(\mathrm{M}+\mathrm{H})^{+}\right]$254.1903, found 254.1903. IR (film) 2920, $2850,1603,1560,1507,1467,1446,1411,1341,757 \mathrm{~cm}^{-1}$.

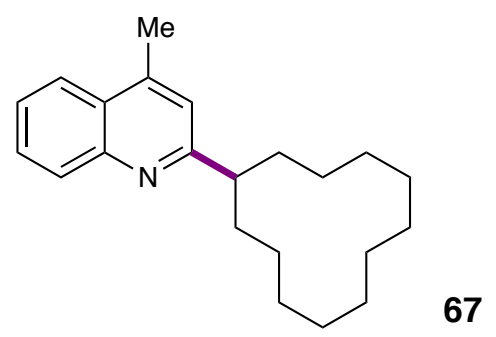

2-Cyclododecyl-4-methylquinoline (67): According to the general procedure (Conditions A), lepidine (71.6 mg, $0.50 \mathrm{mmol}, 1.0$ equiv.), Selectfluor (373.0 mg, 1.0 mmol, 2.0 equiv.), hydrochloric acid (43.0 $\mu 1,0.50 \mathrm{mmol}, 1.0$ equiv.), cyclododecane (841.6 mg, $5.0 \mathrm{mmol}, 10.0$ equiv.), $5.0 \mathrm{~mL}$ of acetone were used. After 16 hours, the reaction mixture was subjected to the workup procedure outlined in the general procedure and purified by flash chromatography (10\% ethyl acetate/hexanes) to provide the title compound as a white solid (139.3 $\mathrm{mg}, 90 \%$ yield).

${ }^{1} \mathrm{H}$ NMR $\left(400 \mathrm{MHz}, \mathrm{CDCl}_{3}\right) \delta 8.09(\mathrm{~d}, J=8.4 \mathrm{~Hz}, 1 \mathrm{H}), 7.94(\mathrm{~d}, J=8.3 \mathrm{~Hz}, 1 \mathrm{H}), 7.69-$ $7.62(\mathrm{~m}, 1 \mathrm{H}), 7.52-7.45(\mathrm{~m}, 1 \mathrm{H}), 7.14(\mathrm{~s}, 1 \mathrm{H}), 3.11(\mathrm{p}, J=6.6 \mathrm{~Hz}, 1 \mathrm{H}), 2.68(\mathrm{~s}, 3 \mathrm{H})$, $1.97-1.85(\mathrm{~m}, 2 \mathrm{H}), 1.78-1.67(\mathrm{~m}, 2 \mathrm{H}), 1.62-1.32(\mathrm{~m}, 18 \mathrm{H}) ;{ }^{13} \mathrm{C} \mathrm{NMR}(101 \mathrm{MHz}$, $\left.\mathrm{CDCl}_{3}\right) \delta 166.66,147.53,143.87,129.50,128.79,126.93,125.27,123.48,121.36,43.01$, $30.13,23.92,23.86,23.67,23.34,22.88,18.78$; HRMS (ESI) $\mathrm{m} / \mathrm{z}$ calculated for $\mathrm{C}_{22} \mathrm{H}_{32} \mathrm{~N}$ 
$\left[(\mathrm{M}+\mathrm{H})^{+}\right]$310.2527, found 310.2528; IR (film) 2926, 2857, 1608, 1563, 1510, 1470, $1442,894,754 \mathrm{~cm}^{-1}$. Spectra data are consistent with those reported in the literature: $J$. Org. Chem. 2000, 65, 2816-2819.
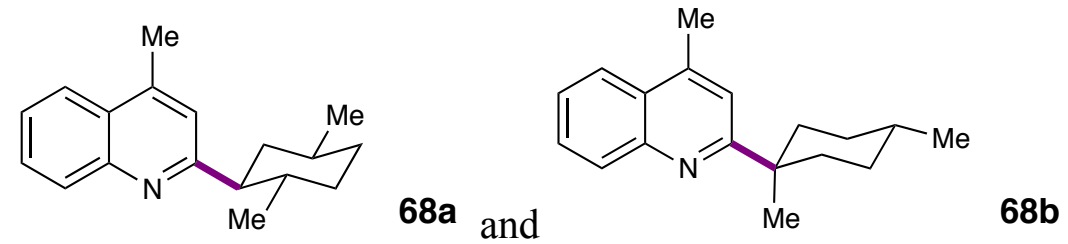

2-(trans-2,5-dimethylcyclohexyl)-4-methylquinoline (68a) and 2-(1,4-

dimethylcyclohexyl)-4-methylquinoline (68b): According to the general procedure (Conditions A), lepidine (71.6 mg, $0.50 \mathrm{mmol}, 1.0$ equiv.), Selectfluor (373.0 mg, 1.0 mmol, 2.0 equiv.), hydrochloric acid ( $43.0 \mu 1,0.50 \mathrm{mmol}, 1.0$ equiv.), trans-1,4dimethylcyclohexane (1.40 ml, $10.0 \mathrm{mmol}, 20.0$ equiv.), $5.0 \mathrm{~mL}$ of acetone were used. After 16 hours, the reaction mixture was subjected to the workup procedure outlined in the general procedure and purified by flash chromatography (10\% ethyl acetate/hexanes) to provide the title compounds as a colorless oil $(52.0 \mathrm{mg}, 41 \%$ yield for $\mathbf{6 8 a})$ and a colorless oil (24.1 mg, 19\% yield for $\mathbf{6 8 b}$ ).

Compound 68a: ${ }^{1} \mathrm{H}$ NMR (400 MHz, $\left.\mathrm{CDCl}_{3}\right) \delta 8.07(\mathrm{~d}, J=8.4 \mathrm{~Hz}, 1 \mathrm{H}), 7.93(\mathrm{~d}, J=8.3$ Hz, 1H), 7.66 (ddd, $J=8.4,6.8,1.5 \mathrm{~Hz}, 1 \mathrm{H}), 7.48$ (ddd, $J=8.2,6.8,1.3 \mathrm{~Hz}, 1 \mathrm{H}), 7.12$ (s, 1H), 2.67 (s, 3H), 2.57 (ddd, $J=12.2,10.6,3.5 \mathrm{~Hz}, 1 \mathrm{H}), 1.93-1.80(\mathrm{~m}, 3 \mathrm{H}), 1.80-1.73$ (m, 1H), $1.65-1.53(\mathrm{~m}, 1 \mathrm{H}), 1.35-1.09(\mathrm{~m}, 3 \mathrm{H}), 0.93(\mathrm{~d}, J=6.5 \mathrm{~Hz}, 3 \mathrm{H}), 0.72(\mathrm{~d}, J=$ $6.3 \mathrm{~Hz}, 3 \mathrm{H}) ;{ }^{13} \mathrm{C} \mathrm{NMR}\left(101 \mathrm{MHz}, \mathrm{CDCl}_{3}\right) \delta 165.79,147.56,144.13,129.40,128.83$, $127.00,125.28,123.50,120.51,54.73,42.47,36.14,35.32,35.10,32.75,22.45,20.28$, 18.79; HRMS (ESI) $\mathrm{m} / \mathrm{z}$ calculated for $\mathrm{C}_{18} \mathrm{H}_{24} \mathrm{~N}\left[(\mathrm{M}+\mathrm{H})^{+}\right]$254.1903, found 254.1902; IR (film) 2948, 2919, 2866, 2845, 1604, 1560, 1507, 1453, 1376, 1342, $758 \mathrm{~cm}^{-1}$. 
Compound 68b: ${ }^{1} \mathrm{H}$ NMR (400 MHz, $\left.\mathrm{CDCl}_{3}\right) \delta 8.06(\mathrm{~d}, J=8.1 \mathrm{~Hz}, 1 \mathrm{H}), 7.94(\mathrm{~d}, J=8.3$ Hz, 1H), 7.66 (t, $J=7.5 \mathrm{~Hz}, 1 \mathrm{H}), 7.49$ (t, $J=7.5 \mathrm{~Hz}, 1 \mathrm{H}), 7.34$ (s, 1H), 2.69 (s, 3H), 2.05 $-1.80(\mathrm{~m}, 4 \mathrm{H}), 1.74-1.64(\mathrm{~m}, 2 \mathrm{H}), 1.49-1.25$ (m, 3H), 1.39 (s, 3H), 0.99 (d, $J=6.3$ $\mathrm{Hz}, 3 \mathrm{H}) ;{ }^{13} \mathrm{C}$ NMR $\left(101 \mathrm{MHz}, \mathrm{CDCl}_{3}\right) \delta 169.86,147.29,143.50,129.87,128.62,126.58$, 125.34, 123.37, 119.05, 40.13, 36.22, 32.19, 30.92, 23.46, 22.36, 19.00; HRMS (ESI) $\mathrm{m} / \mathrm{z}$ calculated for $\mathrm{C}_{18} \mathrm{H}_{24} \mathrm{~N}\left[(\mathrm{M}+\mathrm{H})^{+}\right]$254.1903, found 254.1901; IR (film) 2920, 2852, $1601,1557,1507,1466,1448,1376,757 \mathrm{~cm}^{-1}$.
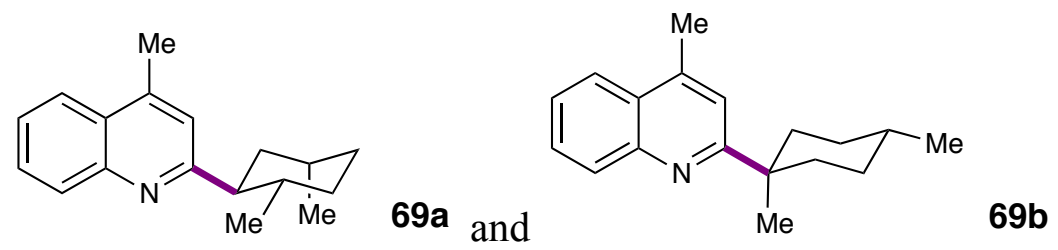

2-(cis-2,5-dimethylcyclohexyl)-4-methylquinoline (69a) and 2-(1,4dimethylcyclohexyl)-4-methylquinoline (69b): According to the general procedure (Conditions A), lepidine (71.6 mg, $0.50 \mathrm{mmol}, 1.0$ equiv.), Selectfluor (373.0 mg, 1.0 mmol, 2.0 equiv.), hydrochloric acid ( $43.0 \mu 1,0.50 \mathrm{mmol}, 1.0$ equiv.), cis-1,4dimethylcyclohexane (1.40 ml, $10.0 \mathrm{mmol}, 20.0$ equiv.), $5.0 \mathrm{~mL}$ of acetone were used. After 16 hours, the reaction mixture was subjected to the workup procedure outlined in the general procedure and purified by flash chromatography (5\% ethyl acetate/hexanes) to provide the title compounds as a colorless oil ( $43.1 \mathrm{mg}, 34 \%$ yield for $69 \mathrm{a})$ and a colorless oil (33.0 mg, 26\% yield for 69b).

Compound 69a: ${ }^{1} \mathrm{H}$ NMR $\left(400 \mathrm{MHz}, \mathrm{CDCl}_{3}\right) \delta 8.09(\mathrm{~d}, J=8.3 \mathrm{~Hz}, 1 \mathrm{H}), 7.94(\mathrm{~d}, J=8.3$ Hz, 1H), 7.66 (ddd, $J=8.4,6.9,1.4 \mathrm{~Hz}, 1 \mathrm{H}), 7.49$ (ddd, $J=8.2,6.9,1.3 \mathrm{~Hz}, 1 \mathrm{H}), 7.14$ (s, 1H), $2.78(\mathrm{td}, J=12.5,4.1 \mathrm{~Hz}, 1 \mathrm{H}), 2.68(\mathrm{~s}, 3 \mathrm{H}), 2.18-2.07(\mathrm{~m}, 1 \mathrm{H}), 1.95-1.81(\mathrm{~m}$, 2H), $1.81-1.65(\mathrm{~m}, 2 \mathrm{H}), 1.65-1.54(\mathrm{~m}, 2 \mathrm{H}), 1.44(\mathrm{~m}, 1 \mathrm{H}), 1.12(\mathrm{~d}, J=7.2 \mathrm{~Hz}, 3 \mathrm{H})$, 
$0.73(\mathrm{~d}, J=6.5 \mathrm{~Hz}, 3 \mathrm{H}) ;{ }^{13} \mathrm{C}$ NMR $\left(101 \mathrm{MHz}, \mathrm{CDCl}_{3}\right) \delta 166.09,147.40,144.25,129.32$, $128.90,127.03,125.36,123.54,120.72,48.67,39.14,37.00,31.65,29.32,27.68,20.54$, 18.85, 18.07; HRMS (ESI) $\mathrm{m} / \mathrm{z}$ calculated for $\mathrm{C}_{18} \mathrm{H}_{24} \mathrm{~N}\left[(\mathrm{M}+\mathrm{H})^{+}\right]$254.1903, found 254.1902. IR (film) 2918, 2851, 1603, 1560, 1507, 1449, 1377, $757 \mathrm{~cm}^{-1}$.

Compound 69b: ${ }^{1} \mathrm{H}$ NMR $\left(400 \mathrm{MHz}, \mathrm{CDCl}_{3}\right) \delta 8.06(\mathrm{~d}, J=8.0 \mathrm{~Hz}, 1 \mathrm{H}), 7.94(\mathrm{~d}, J=8.1$ Hz, 1H), 7.66 (t, $J=7.5 \mathrm{~Hz}, 1 \mathrm{H}), 7.49$ (t, $J=7.5 \mathrm{~Hz}, 1 \mathrm{H}), 7.34$ (s, 1H), 2.69 (s, 3H), 2.05 $-1.80(\mathrm{~m}, 4 \mathrm{H}), 1.74-1.64(\mathrm{~m}, 2 \mathrm{H}), 1.49-1.25(\mathrm{~m}, 3 \mathrm{H}), 1.39(\mathrm{~s}, 3 \mathrm{H}), 0.99(\mathrm{~d}, J=6.3$ $\mathrm{Hz}, 3 \mathrm{H}) ;{ }^{13} \mathrm{C} \mathrm{NMR}\left(101 \mathrm{MHz}, \mathrm{CDCl}_{3}\right) \delta 169.86,147.29,143.49,129.90,128.63,126.58$, 125.34, 123.37, 119.05, 40.13, 36.22, 32.19, 30.92, 23.46, 22.36, 19.00; HRMS (ESI) $\mathrm{m} / \mathrm{z}$ calculated for $\mathrm{C}_{18} \mathrm{H}_{24} \mathrm{~N}\left[(\mathrm{M}+\mathrm{H})^{+}\right]$254.1903, found 254.1901; IR (film) 2920, 2852, $1601,1557,1507,1466,1448,1376,757 \mathrm{~cm}^{-1}$.

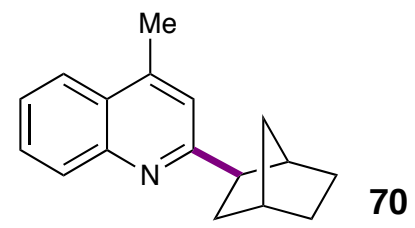

2-(Bicyclo[2.2.1]heptan-2-yl)-4-methylquinoline (70): According to the general procedure (Conditions A), lepidine (71.6 mg, $0.50 \mathrm{mmol}, 1.0$ equiv.), Selectfluor (373.0 $\mathrm{mg}, 1.0 \mathrm{mmol}, 2.0$ equiv.), hydrochloric acid (43.0 $\mu \mathrm{l}, 0.50 \mathrm{mmol}, 1.0$ equiv.), bicyclo[2.2.1]heptane (721.3 mg, $7.5 \mathrm{mmol}, 15.0$ equiv.), $5.0 \mathrm{~mL}$ of acetone were used. After 16 hours, the reaction mixture was subjected to the workup procedure outlined in the general procedure and purified by flash chromatography ( $20 \%$ ethyl acetate/hexanes) to provide the title compound as a colorless oil ( $103.3 \mathrm{mg}, 87 \%$ yield).

${ }^{1} \mathrm{H}$ NMR (400 MHz, $\left.\mathrm{CDCl}_{3}\right) \delta 8.07(\mathrm{~d}, J=8.3 \mathrm{~Hz}, 1 \mathrm{H}), 7.92(\mathrm{~d}, J=7.7 \mathrm{~Hz}, 1 \mathrm{H}), 7.66$

(ddd, $J=8.4,6.8,1.5 \mathrm{~Hz}, 1 \mathrm{H}), 7.48$ (ddd, $J=8.2,6.8,1.3 \mathrm{~Hz}, 1 \mathrm{H}$ ), 7.18 (s, 1H), 3.01 (dd, $J=9.1,5.4 \mathrm{~Hz}, 1 \mathrm{H}), 2.66(\mathrm{~s}, 3 \mathrm{H}), 2.60-2.55(\mathrm{~m}, 1 \mathrm{H}), 2.46-2.39(\mathrm{~m}, 1 \mathrm{H}), 2.32-2.22$ 
(m, 1H), $1.79-1.56(\mathrm{~m}, 4 \mathrm{H}), 1.53-1.44(\mathrm{~m}, 1 \mathrm{H}), 1.39-1.31(\mathrm{~m}, 1 \mathrm{H}), 1.23-1.16(\mathrm{~d}, J$ $=9.6 \mathrm{~Hz}, 1 \mathrm{H}) ;{ }^{13} \mathrm{C} \mathrm{NMR}\left(101 \mathrm{MHz}, \mathrm{CDCl}_{3}\right) \delta 165.45,147.37,143.63,129.63,128.71$, $126.67,125.20,123.39,121.49,49.97,42.99,36.69,36.18,36.00,30.47,29.11,18.69$; HRMS (ESI) $\mathrm{m} / \mathrm{z}$ calculated for $\mathrm{C}_{17} \mathrm{H}_{20} \mathrm{~N}\left[(\mathrm{M}+\mathrm{H})^{+}\right] 238.1590$, found 238.1590; IR (film) $2949,2867,1604,1560,1508,1448,837,757 \mathrm{~cm}^{-1}$.
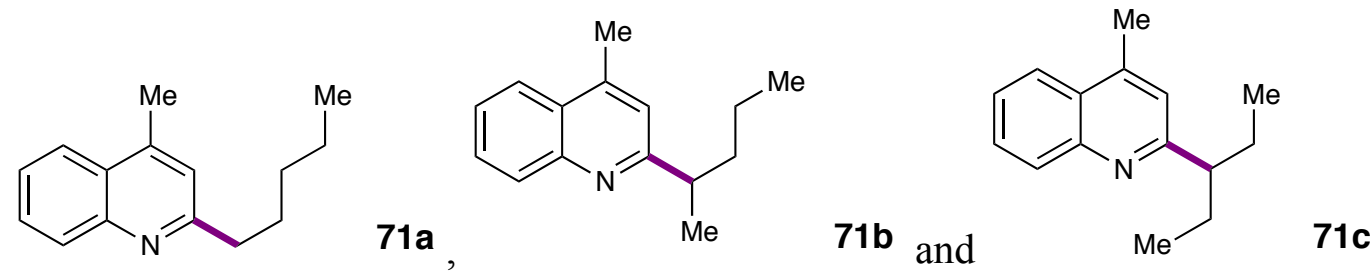

4-Methyl-2-pentylquinoline (71a), 4-Methyl-2-(pentan-2-yl)quinoline (71b) and 4Methyl-2-(pentan-3-yl)quinoline (71c): According to the general procedure (Conditions A), lepidine (71.6 mg, $0.50 \mathrm{mmol}, 1.0$ equiv.), Selectfluor (466.3 mg, $1.25 \mathrm{mmol}, 2.5$ equiv.), hydrochloric acid ( $43.0 \mu 1,0.50 \mathrm{mmol}, 1.0$ equiv.), pentane (1.20 ml, $10.0 \mathrm{mmol}$, 20.0 equiv.), $5.0 \mathrm{~mL}$ of acetone were used. After 16 hours, the reaction mixture was subjected to the workup procedure outlined in the general procedure and purified by flash chromatography (5\% ethyl acetate/hexanes) to provide the title compounds as a colorless oil mixture of $74.7 \mathrm{mg}, 70 \%$ total yield ( $8 \%$ yield for $\mathbf{7 1 a}$; $47 \%$ yield for $\mathbf{7 1 b} ; 15 \%$ yield for 71c). HRMS (ESI) $\mathrm{m} / \mathrm{z}$ calculated for $\mathrm{C}_{15} \mathrm{H}_{20} \mathrm{~N}\left[(\mathrm{M}+\mathrm{H})^{+}\right]$214.1590, found 214.1590; IR (film) 2957, 2928, 1603, 1560, 1507, 1448, 1409, $758 \mathrm{~cm}^{-1}$.

Compound 71a: ${ }^{1} \mathrm{H}$ NMR $\left(400 \mathrm{MHz}, \mathrm{CDCl}_{3}\right) \delta 8.07(\mathrm{t}, J=7.9 \mathrm{~Hz}, 1 \mathrm{H}), 7.93(\mathrm{~d}, J=8.2$ Hz, 2H), 7.66 (t, $J=7.6 \mathrm{~Hz}, 1 \mathrm{H}), 7.48(\mathrm{t}, J=7.6 \mathrm{~Hz}, 1 \mathrm{H}), 7.13(\mathrm{~s}, 1 \mathrm{H}), 2.92$ (t, $J=7.9$ Hz, 2H), 2.66 (s, 1H), $1.85-1.73(\mathrm{~m}, 2 \mathrm{H}), 1.43-1.32(\mathrm{~m}, 4 \mathrm{H}), 0.90$ (t, $J=7.3 \mathrm{~Hz}, 3 \mathrm{H})$; ${ }^{13} \mathrm{C}$ NMR (101 MHz, $\left.\mathrm{CDCl}_{3}\right) \delta 162.70,147.54,144.19,129.24,128.94,126.70,125.30$, 
$123.48,121.99,39.15,31.75,29.73,22.52,18.61,13.96$. Spectra data are consistent with those reported in the literature: Org. Lett. 2018, 20, 4686-4690.

Compound 71b: ${ }^{1} \mathrm{H}$ NMR (400 MHz, $\left.\mathrm{CDCl}_{3}\right) \delta 8.07(\mathrm{t}, J=7.9 \mathrm{~Hz}, 1 \mathrm{H}), 7.93(\mathrm{~d}, J=8.2$ Hz, 2H), 7.66 (t, $J=7.6 \mathrm{~Hz}, 1 \mathrm{H}), 7.48$ (t, $J=7.6 \mathrm{~Hz}, 1 \mathrm{H}), 7.13(\mathrm{~s}, 1 \mathrm{H}), 3.06$ (h, $J=7.1$ Hz, 1H), $2.67(\mathrm{~s}, 1 \mathrm{H}), 1.85-1.73(\mathrm{~m}, 1 \mathrm{H}), 1.70-1.59(\mathrm{~m}, 1 \mathrm{H}), 1.43-1.32(\mathrm{~m}, 1 \mathrm{H}), 1.35$ $(\mathrm{d}, J=7.0 \mathrm{~Hz}, 3 \mathrm{H}), 1.28-1.18(\mathrm{~m}, 1 \mathrm{H}), 0.90(\mathrm{t}, J=7.3 \mathrm{~Hz}, 3 \mathrm{H}) ;{ }^{13} \mathrm{C} \mathrm{NMR}(101 \mathrm{MHz}$ $\left.\mathrm{CDCl}_{3}\right) \delta 166.81,147.47,144.19,129.40,128.83,126.95,125.30,123.48,120.09,42.57$, $39.23,20.81,20.67,18.77,14.13$.

Compound 71c: ${ }^{1} \mathrm{H}$ NMR $\left(400 \mathrm{MHz}, \mathrm{CDCl}_{3}\right) \delta 8.09(\mathrm{t}, J=7.9 \mathrm{~Hz}, 1 \mathrm{H}), 7.93(\mathrm{~d}, J=8.2$ $\mathrm{Hz}, 2 \mathrm{H}), 7.66$ (t, $J=7.6 \mathrm{~Hz}, 1 \mathrm{H}), 7.48$ (t, $J=7.6 \mathrm{~Hz}, 1 \mathrm{H}), 7.10$ (s, 1H), 2.74 (p, $J=7.4$ Hz, 1H), $2.67(\mathrm{~s}, 1 \mathrm{H}), 1.85-1.73(\mathrm{~m}, 4 \mathrm{H}), 0.83(\mathrm{t}, J=7.4 \mathrm{~Hz}, 6 \mathrm{H}) ;{ }^{13} \mathrm{C} \mathrm{NMR}(101 \mathrm{MHz}$, $\left.\mathrm{CDCl}_{3}\right) \delta 165.53,147.58,143.93,129.47,128.77,126.98,125.28,123.50,120.66,52.09$, $28.17,18.77,12.17$. Spectra data are consistent with those reported in the literature: Org. Lett. 2018, 20, 4686-4690.
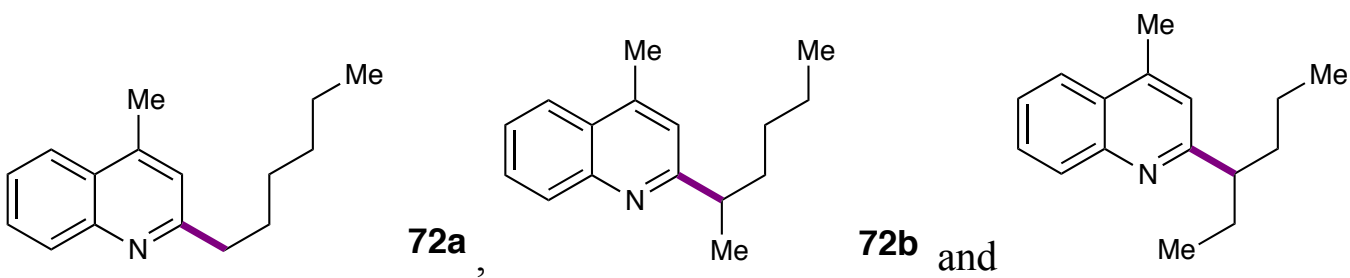

72c

2-Hexyl-4-methylquinoline (72a), 2-(Hexan-2-yl)-4-methylquinoline (72b) and 2(Hexan-3-yl)-4-methylquinoline (72c): According to the general procedure (Conditions A), lepidine (71.6 mg, $0.50 \mathrm{mmol}, 1.0$ equiv.), Selectfluor (373.0 mg, $1.0 \mathrm{mmol}, 2.0$ equiv.), hydrochloric acid ( $43.0 \mu \mathrm{l}, 0.50 \mathrm{mmol}, 1.0$ equiv.), hexane (1.30 ml, $10.0 \mathrm{mmol}$, 20.0 equiv.), $5.0 \mathrm{~mL}$ of acetone were used. After 16 hours, the reaction mixture was subjected to the workup procedure outlined in the general procedure and purified by flash 
chromatography ( $10 \%$ ethyl acetate/hexanes) to provide the title compounds as a colorless oil mixture of $69.5 \mathrm{mg}, 61 \%$ total yield $(5 \%$ yield for $\mathbf{7 2 a}$; $35 \%$ yield for $\mathbf{7 2 b}$; $21 \%$ yield for $72 \mathrm{c}$ ). HRMS (ESI) $\mathrm{m} / \mathrm{z}$ calculated for $\mathrm{C}_{16} \mathrm{H}_{22} \mathrm{~N}\left[(\mathrm{M}+\mathrm{H})^{+}\right] 228.1747$, found 228.1747; IR (film) 2957, 2928, 2870, 1603, 1560, 1507, 1449, $758 \mathrm{~cm}^{-1}$.

Compound 72a: ${ }^{1} \mathrm{H}$ NMR $\left(400 \mathrm{MHz}, \mathrm{CDCl}_{3}\right) \delta 8.06(\mathrm{~d}, J=8.5 \mathrm{~Hz}, 1 \mathrm{H}), 7.93(\mathrm{~d}, J=8.3$ Hz, 1H), $7.66(\mathrm{t}, J=7.6 \mathrm{~Hz}, 1 \mathrm{H}), 7.48(\mathrm{t}, J=7.2 \mathrm{~Hz}, 1 \mathrm{H}), 7.13(\mathrm{~s}, 1 \mathrm{H}), 2.92(\mathrm{t}, J=7.8$ Hz, 2H), $2.65(\mathrm{~s}, 3 \mathrm{H}), 1.87-1.61(\mathrm{~m}, 2 \mathrm{H}), 1.46-1.25(\mathrm{~m}, 6 \mathrm{H}), 0.87$ (t, $J=7.0 \mathrm{~Hz}, 3 \mathrm{H})$; ${ }^{13} \mathrm{C}$ NMR $\left(101 \mathrm{MHz}, \mathrm{CDCl}_{3}\right) \delta 162.69,147.49,144.09,129.21,128.92,126.69,125.28$, $123.47,121.97,39.20,31.68,29.99,29.22,22.50,18.59,14.01$. Spectra data are consistent with those reported in the literature: J. Am. Chem. Soc. 2017, 139, 1431514321.

Compound 72b: ${ }^{1} \mathrm{H}$ NMR (400 MHz, $\left.\mathrm{CDCl}_{3}\right) \delta 8.07(\mathrm{~d}, J=8.4 \mathrm{~Hz}, 1 \mathrm{H}), 7.93(\mathrm{~d}, J=8.3$ Hz, 1H), 7.66 (t, $J=7.6 \mathrm{~Hz}, 1 \mathrm{H}), 7.48$ (t, $J=7.2 \mathrm{~Hz}, 1 \mathrm{H}), 7.13$ (s, 1H), 3.04 (h, $J=7.0$ $\mathrm{Hz}, 1 \mathrm{H}), 2.67(\mathrm{~s}, 3 \mathrm{H}), 1.87-1.61(\mathrm{~m}, 3 \mathrm{H}), 1.39-1.24(\mathrm{~m}, 2 \mathrm{H}), 1.36(\mathrm{~d}, J=7.0 \mathrm{~Hz}, 3 \mathrm{H})$, $1.24-1.11(\mathrm{~m}, 1 \mathrm{H}), 0.85(\mathrm{t}, J=7.0 \mathrm{~Hz}, 3 \mathrm{H}) ;{ }^{13} \mathrm{C} \mathrm{NMR}\left(101 \mathrm{MHz}, \mathrm{CDCl}_{3}\right) \delta 166.83$, $147.49,144.15,129.42,128.81,126.95,125.28,123.47,120.08,42.82,36.73,29.89$, $22.75,20.72,18.76,13.95$.

Compound 72c: ${ }^{1} \mathrm{H}$ NMR $\left(400 \mathrm{MHz}, \mathrm{CDCl}_{3}\right) \delta 8.08(\mathrm{~d}, J=8.5 \mathrm{~Hz}, 1 \mathrm{H}), 7.93(\mathrm{~d}, J=8.3$ Hz, 1H), 7.66 (t, $J=7.6 \mathrm{~Hz}, 1 \mathrm{H}), 7.48$ (t, $J=7.2 \mathrm{~Hz}, 1 \mathrm{H}), 7.10$ (s, 1H), 2.84 (p, $J=7.4$ Hz, 1H), 2.67 (s, 3H), $1.87-1.61(\mathrm{~m}, 2 \mathrm{H}), 1.39-1.24(\mathrm{~m}, 3 \mathrm{H}), 1.24-1.11(\mathrm{~m}, 1 \mathrm{H}), 0.87$ (t, $J=7.0 \mathrm{~Hz}, 3 \mathrm{H}), 0.83(\mathrm{t}, J=7.0 \mathrm{~Hz}, 3 \mathrm{H}) ;{ }^{13} \mathrm{C} \mathrm{NMR}\left(101 \mathrm{MHz}, \mathrm{CDCl}_{3}\right) \delta 165.71$, $147.54,143.88,129.47,128.74,126.96,125.25,123.47,120.64,50.20,37.61,28.47$, $20.78,18.76,14.19,12.17$. 


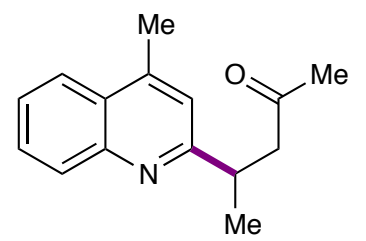

73

4-(4-Methylquinolin-2-yl)pentan-2-one (73): According to the general procedure (Conditions A), lepidine (71.6 mg, $0.50 \mathrm{mmol}, 1.0$ equiv.), Selectfluor (466.3 mg, 1.25 mmol, 2.5 equiv.), hydrochloric acid ( $43.0 \mu \mathrm{l}, 0.50 \mathrm{mmol}, 1.0$ equiv.), 2-pentanone (1.4 $\mathrm{ml}, 12.5 \mathrm{mmol}, 25.0$ equiv.), $5.0 \mathrm{~mL}$ of acetone were used. After 24 hours, the reaction mixture was subjected to the workup procedure outlined in the general procedure and purified by flash chromatography (50\% ethyl acetate/hexanes) to provide the title compound as a colorless oil ( $56.9 \mathrm{mg}, 50 \%$ yield).

${ }^{1} \mathrm{H}$ NMR (400 MHz, $\left.\mathrm{CDCl}_{3}\right) \delta 8.01(\mathrm{~d}, J=8.4 \mathrm{~Hz}, 1 \mathrm{H}), 7.92(\mathrm{~d}, J=8.3 \mathrm{~Hz}, 1 \mathrm{H}), 7.65$ (ddd, $J=8.4,6.8,1.4 \mathrm{~Hz}, 1 \mathrm{H}), 7.48$ (ddd, $J=8.2,6.8,1.2 \mathrm{~Hz}, 1 \mathrm{H}), 7.17$ (s, 1H), 3.60 (h, $J=7.1 \mathrm{~Hz}, 1 \mathrm{H}), 3.30(\mathrm{dd}, J=16.8,7.8 \mathrm{~Hz}, 1 \mathrm{H}), 2.71(\mathrm{dd}, J=16.9,6.3 \mathrm{~Hz}, 1 \mathrm{H}), 2.66$ (s, 3H), $2.18(\mathrm{~s}, 3 \mathrm{H}), 1.36(\mathrm{~d}, J=7.1 \mathrm{~Hz}, 3 \mathrm{H}) ;{ }^{13} \mathrm{C} \mathrm{NMR}\left(101 \mathrm{MHz}, \mathrm{CDCl}_{3}\right) \delta 208.27$, $164.59,147.33,144.43,129.25,128.89,126.95,125.48,123.53,121.36,49.07,37.49$, 30.52, 20.91, 18.67; HRMS (ESI) m/z calculated for $\mathrm{C}_{15} \mathrm{H}_{18} \mathrm{ON}\left[(\mathrm{M}+\mathrm{H})^{+}\right] 228.1383$, found 228.1381. IR (film) 2964, 1715, 1604, 1508, 1407, 1358, 1164, $761 \mathrm{~cm}^{-1}$.
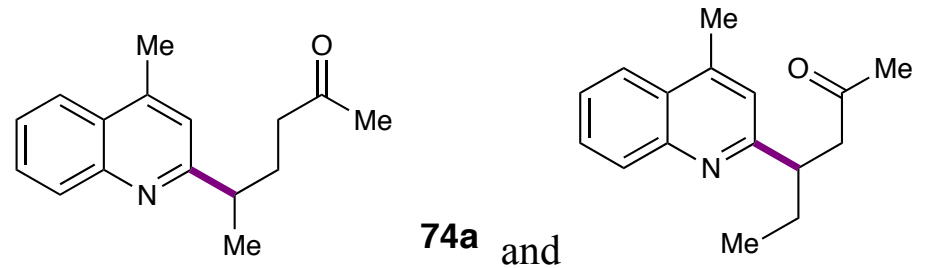

74b

5-(4-Methylquinolin-2-yl)hexan-2-one (74a) and 4-(4-Methylquinolin-2-yl)hexan-2one (74b): According to the general procedure (Conditions A), lepidine (71.6 mg, 0.50 mmol, 1.0 equiv.), Selectfluor (373.0 mg, $1.0 \mathrm{mmol}, 2.0$ equiv.), hydrochloric acid (43.0 
$\mu 1,0.50 \mathrm{mmol}, 1.0$ equiv.), 2-hexanone ( $1.50 \mathrm{ml}, 12.5 \mathrm{mmol}, 25.0$ equiv.), $5.0 \mathrm{~mL}$ of acetone were used. After 24 hours, the reaction mixture was subjected to the workup procedure outlined in the general procedure and purified by flash chromatography $(5 \%$ ethyl acetate/hexanes) to provide the title compounds as a colorless oil (47.1 mg, 39\% yield for 74a) and a colorless oil (24.1 $\mathrm{mg}, 20 \%$ yield for $\mathbf{7 4 b}$ ).

Compound 74a: ${ }^{1} \mathrm{H}$ NMR (400 MHz, $\left.\mathrm{CDCl}_{3}\right) \delta 8.04(\mathrm{~d}, J=8.1 \mathrm{~Hz}, 1 \mathrm{H}), 7.94(\mathrm{~d}, J=8.3$ Hz, 1H), 7.67 (ddd, $J=8.4,6.9,1.5 \mathrm{~Hz}, 1 \mathrm{H}), 7.50$ (ddd, $J=8.2,6.8,1.3 \mathrm{~Hz}, 1 \mathrm{H}$ ), 7.13 (s, 1H), $3.09-2.96$ (m, 1H), 2.68 (s, 3H), 2.44 (ddd, $J=17.3,9.6,6.2 \mathrm{~Hz}, 1 \mathrm{H}), 2.31$ (ddd, $J$ $=17.3,9.7,5.4 \mathrm{~Hz}, 1 \mathrm{H}), 2.14-1.93(\mathrm{~m}, 2 \mathrm{H}), 2.05(\mathrm{~s}, 3 \mathrm{H}), 1.36(\mathrm{~d}, J=7.0 \mathrm{~Hz}, 3 \mathrm{H}) ;{ }^{13} \mathrm{C}$ NMR (101 MHz, $\left.\mathrm{CDCl}_{3}\right) \delta$ 208.93, 165.46, 147.34, 144.72, 129.35, 129.05, 127.03, 125.58, 123.57, 120.09, 42.02, 41.74, 30.49, 29.90, 20.90, 18.81; HRMS (ESI) m/z calculated for $\mathrm{C}_{16} \mathrm{H}_{20} \mathrm{ON}\left[(\mathrm{M}+\mathrm{H})^{+}\right]$242.1539, found 242.1537; IR (film) 2976, 2871, $1603,1560,1507,1466,1347,1202,759 \mathrm{~cm}^{-1}$.

Compound 74b: ${ }^{1} \mathrm{H}$ NMR (400 MHz, $\left.\mathrm{CDCl}_{3}\right) \delta 8.02(\mathrm{~d}, J=8.4 \mathrm{~Hz}, 1 \mathrm{H}), 7.93(\mathrm{~d}, J=8.2$ Hz, 1H), 7.65 (ddd, $J=8.4,6.9,1.5 \mathrm{~Hz}, 1 \mathrm{H}), 7.49$ (ddd, $J=8.2,6.9,1.3 \mathrm{~Hz}, 1 \mathrm{H}), 7.16$ (s, 1H), 3.39 (tt, $J=7.8,5.6 \mathrm{~Hz}, 1 \mathrm{H}), 3.30(\mathrm{dd}, J=16.5,8.9 \mathrm{~Hz}, 1 \mathrm{H}), 2.76$ (dd, $J=16.5,5.1$ Hz, 1H), 2.67 (s, 3H), 2.13 (s, 3H), 1.83 (dq, $J=14.9,7.5 \mathrm{~Hz}, 1 \mathrm{H}), 1.73$ (dq, $J=13.7,7.1$ $\mathrm{Hz}, 1 \mathrm{H}), 0.85(\mathrm{t}, J=7.4 \mathrm{~Hz}, 3 \mathrm{H}) ;{ }^{13} \mathrm{C} \mathrm{NMR}\left(101 \mathrm{MHz}, \mathrm{CDCl}_{3}\right) \delta 208.59,163.73,147.55$, $144.07,129.39,128.86,127.05,125.49,123.62,122.48,47.62,44.61,30.61,28.49$, 18.73, 11.91; HRMS (ESI) m/z calculated for $\mathrm{C}_{16} \mathrm{H}_{20} \mathrm{ON}\left[(\mathrm{M}+\mathrm{H})^{+}\right] 242.1539$, found 242.1537. IR (film) 2962, 2930, 1715, 1603, 1560, 1508, 1163, 760, $732 \mathrm{~cm}^{-1}$. 


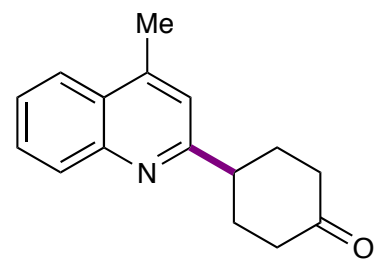

$75 a$ and

4-(4-Methylquinolin-2-yl)cyclohexan-1-one (75a) and 3-(4-Methylquinolin-2-

yl)cyclohexan-1-one (75b): According to the general procedure (Conditions A), lepidine (71.6 mg, $0.50 \mathrm{mmol}, 1.0$ equiv.), Selectfluor (466.3 mg, $1.25 \mathrm{mmol}, 2.5$ equiv.), hydrochloric acid (43.0 $\mu$ l, $0.50 \mathrm{mmol}, 1.0$ equiv.), cyclohexanone (1.25 ml, $12.5 \mathrm{mmol}$, 25.0 equiv.), $5.0 \mathrm{~mL}$ of acetone were used. After 24 hours, the reaction mixture was subjected to the workup procedure outlined in the general procedure and purified by flash chromatography ( $50 \%$ ethyl acetate/hexanes) to provide the title compounds as a colorless oil (6.0 mg, 5\% yield for 75a) and a colorless oil $(73.0 \mathrm{mg}, 61 \%$ yield for $\mathbf{7 5 b})$. Compound 75a: ${ }^{1} \mathrm{H}$ NMR $\left(400 \mathrm{MHz}, \mathrm{CDCl}_{3}\right) \delta 8.04(\mathrm{~d}, J=8.4 \mathrm{~Hz}, 1 \mathrm{H}), 7.96(\mathrm{~d}, J=8.3$ $\mathrm{Hz}, 1 \mathrm{H}), 7.69(\mathrm{t}, J=7.6 \mathrm{~Hz}, 1 \mathrm{H}), 7.53(\mathrm{t}, J=7.6 \mathrm{~Hz}, 1 \mathrm{H}), 7.18(\mathrm{~s}, 1 \mathrm{H}), 3.36(\mathrm{tt}, J=11.5$, $3.8 \mathrm{~Hz}, 1 \mathrm{H}), 2.70(\mathrm{~s}, 3 \mathrm{H}), 2.63-2.47(\mathrm{~m}, 4 \mathrm{H}), 2.41-2.30(\mathrm{~m}, 2 \mathrm{H}), 2.25-2.13(\mathrm{~m}, 2 \mathrm{H})$; ${ }^{13} \mathrm{C}$ NMR $\left(101 \mathrm{MHz}, \mathrm{CDCl}_{3}\right) \delta 211.14,163.44,147.52,144.84,129.47,129.26,127.10$, 125.81, 123.62, 119.97, 44.95, 40.94, 32.23, 18.85; HRMS (ESI) m/z calculated for $\mathrm{C}_{16} \mathrm{H}_{18} \mathrm{ON}\left[(\mathrm{M}+\mathrm{H})^{+}\right]$240.1383, found 240.1382; IR (film) 2948, 2866, 1716, 1602, 1561, $1508,1148,1414,1162,761,732 \mathrm{~cm}^{-1}$.

Compound 75b: ${ }^{1} \mathrm{H}$ NMR $\left(400 \mathrm{MHz}, \mathrm{CDCl}_{3}\right) \delta 8.02(\mathrm{~d}, J=8.4 \mathrm{~Hz}, 1 \mathrm{H}), 7.93(\mathrm{~d}, J=8.3$ $\mathrm{Hz}, 1 \mathrm{H}), 7.66(\mathrm{t}, J=7.6 \mathrm{~Hz}, 1 \mathrm{H}), 7.50(\mathrm{t}, J=7.6 \mathrm{~Hz}, 1 \mathrm{H}), 7.11(\mathrm{~s}, 1 \mathrm{H}), 3.30(\mathrm{tt}, J=11.5$, $3.9 \mathrm{~Hz}, 1 \mathrm{H}), 2.95(\mathrm{dd}, J=14.3,11.9 \mathrm{~Hz}, 1 \mathrm{H}), 2.67(\mathrm{~s}, 3 \mathrm{H}), 2.66-2.60(\mathrm{~m}, 1 \mathrm{H}), 2.48-$ $2.38(\mathrm{~m}, 2 \mathrm{H}), 2.19-2.09(\mathrm{~m}, 2 \mathrm{H}), 2.08-1.97(\mathrm{~m}, 1 \mathrm{H}), 1.86-1.75(\mathrm{~m}, 1 \mathrm{H}) ;{ }^{13} \mathrm{C} \mathrm{NMR}$ $\left(101 \mathrm{MHz}, \mathrm{CDCl}_{3}\right) \delta 211.35,162.44,147.44,144.84,129.41,129.13,127.00,125.75$ 
123.53, 120.60, 46.73, 46.42, 41.10, 31.40, 25.19, 18.70; HRMS (ESI) m/z calculated for $\mathrm{C}_{16} \mathrm{H}_{18} \mathrm{ON}\left[(\mathrm{M}+\mathrm{H})^{+}\right] 240.1383$, found 240.1380; IR (film) 2940, 2863, 1707, 1601, 1561, $1507,1448,1414,1312,760 \mathrm{~cm}^{-1}$.
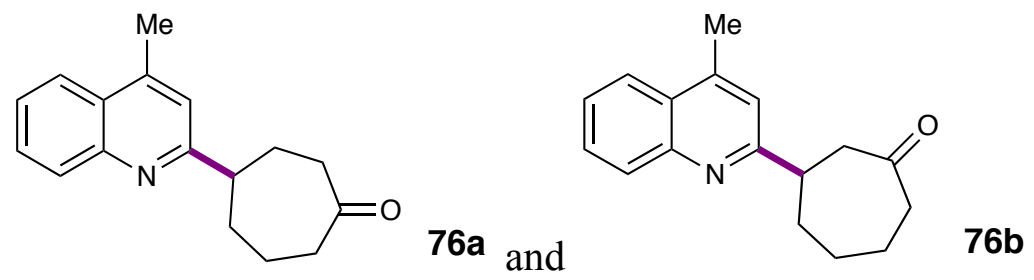

4-(4-Methylquinolin-2-yl)cycloheptan-1-one (76a) and 3-(4-Methylquinolin-2-

yl)cycloheptan-1-one (76b): According to the general procedure (Conditions A), lepidine (71.6 mg, $0.50 \mathrm{mmol}, 1.0$ equiv.), Selectfluor (466.3 mg, $1.25 \mathrm{mmol}, 2.5$ equiv.), hydrochloric acid (43.0 $\mu \mathrm{l}, 0.50 \mathrm{mmol}, 1.0$ equiv.), cycloheptanone $(1.50 \mathrm{ml}, 12.5 \mathrm{mmol}$, 25.0 equiv.), $5.0 \mathrm{~mL}$ of acetone were used. After 24 hours, the reaction mixture was subjected to the workup procedure outlined in the general procedure and purified by flash chromatography (50\% ethyl acetate/hexanes) to provide the title compounds as a white solid (76.0 mg, 60\% yield for 76a) and a colorless oil (26.6 mg, $21 \%$ yield for $76 \mathbf{b}$ ).

Compound 76a: ${ }^{1} \mathrm{H}$ NMR (400 MHz, $\left.\mathrm{CDCl}_{3}\right) \delta 8.04(\mathrm{~d}, J=8.4 \mathrm{~Hz}, 1 \mathrm{H}), 7.95(\mathrm{~d}, J=8.2$ Hz, 1H), 7.68 (ddd, $J=8.4,6.8,1.5 \mathrm{~Hz}, 1 \mathrm{H}), 7.51$ (ddd, $J=8.2,6.8,1.3 \mathrm{~Hz}, 1 \mathrm{H}), 7.12$ (s, 1H), $3.06(\mathrm{tt}, J=11.2,3.4 \mathrm{~Hz}, 1 \mathrm{H}), 2.80-2.72(\mathrm{~m}, 1 \mathrm{H}), 2.69(\mathrm{~s}, 3 \mathrm{H}), 2.68-2.60(\mathrm{~m}$, $3 \mathrm{H}), 2.31-2.21(\mathrm{~m}, 1 \mathrm{H}), 2.21-2.13(\mathrm{~m}, 1 \mathrm{H}), 2.13-2.01(\mathrm{~m}, 2 \mathrm{H}), 1.90-1.77(\mathrm{~m}, 2 \mathrm{H})$; ${ }^{13} \mathrm{C}$ NMR $\left(101 \mathrm{MHz}, \mathrm{CDCl}_{3}\right) \delta 214.60,165.43,147.30,145.01,129.32,129.26,127.00$, $125.73,123.59,120.04,51.02,43.77,42.71,36.56,30.03,23.53,18.84 ;$ HRMS (ESI) $\mathrm{m} / \mathrm{z}$ calculated for $\mathrm{C}_{17} \mathrm{H}_{20} \mathrm{ON}\left[(\mathrm{M}+\mathrm{H})^{+}\right]$254.1539, found 254.1537; IR (film) 2930, 2866, $1690,1604,1560,1450,1337,1164,1035,757 \mathrm{~cm}^{-1}$. 
Compound 76b: ${ }^{1} \mathrm{H}$ NMR (400 MHz, $\left.\mathrm{CDCl}_{3}\right) \delta 8.02(\mathrm{~d}, J=8.4 \mathrm{~Hz}, 1 \mathrm{H}), 7.93(\mathrm{~d}, J=8.3$ Hz, 1H), 7.66 (ddd, $J=8.4,6.8,1.5 \mathrm{~Hz}, 1 \mathrm{H}), 7.50$ (ddd, $J=8.2,6.8,1.3 \mathrm{~Hz}, 1 \mathrm{H}), 7.10$ (s, 1H), 3.27 (tt, $J=10.9,2.7 \mathrm{~Hz}, 1 \mathrm{H}), 3.11$ (dd, $J=14.9,11.4 \mathrm{~Hz}, 1 \mathrm{H}), 2.79$ (ddd, $J=14.9$, 2.8, $1.7 \mathrm{~Hz}, 1 \mathrm{H}), 2.66$ (s, 3H), $2.63(\mathrm{dd}, J=7.8,4.6 \mathrm{~Hz}, 2 \mathrm{H}), 2.22-2.13(\mathrm{~m}, 1 \mathrm{H}), 2.07-$ $1.87(\mathrm{~m}, 3 \mathrm{H}), 1.74(\mathrm{tt}, J=14.7,6.5 \mathrm{~Hz}, 1 \mathrm{H}), 1.65-1.53(\mathrm{~m}, 1 \mathrm{H}) ;{ }^{13} \mathrm{C} \mathrm{NMR}(101 \mathrm{MHz}$, $\left.\mathrm{CDCl}_{3}\right) \delta 213.58,164.55,147.22,145.00,129.30,129.17,126.92,125.73,123.53$, 120.20, 49.17, 44.64, 43.96, 37.09, 28.98, 24.15, 18.74; HRMS (ESI) m/z calculated for $\mathrm{C}_{17} \mathrm{H}_{20} \mathrm{ON}\left[(\mathrm{M}+\mathrm{H})^{+}\right]$254.1539, found 254.1537. IR (film) 2926, 2855, 1698, 1602, 1561, $1508,1446,1411,1344,760 \mathrm{~cm}^{-1}$.<smiles>COC(=O)CCCc1cc(C)c2ccccc2n1</smiles><smiles>COC(=O)CC(C)c1cc([N+](=O)[O-])c2ccccc2n1</smiles>

$77 b$

Methyl 4-(4-methylquinolin-2-yl)butanoate (77a) and Methyl 3-(4-methylquinolin-2yl)butanoate (77b): According to the general procedure (Conditions A), lepidine (71.6 $\mathrm{mg}, 0.50 \mathrm{mmol}, 1.0$ equiv.), Selectfluor (466.3 mg, $1.25 \mathrm{mmol}, 2.5$ equiv.), hydrochloric acid (43.0 $\mu 1,0.50 \mathrm{mmol}, 1.0$ equiv.), methyl butyrate (1.40 ml, $12.5 \mathrm{mmol}, 25.0$ equiv.), $5.0 \mathrm{~mL}$ of acetone were used. After 24 hours, the reaction mixture was subjected to the workup procedure outlined in the general procedure and purified by flash chromatography ( $30 \%$ ethyl acetate/hexanes) to provide the title compounds as a colorless oil (30.4 mg, 25\% yield for 77a) and a colorless oil (45.0 mg, 37\% yield for 77b).

Compound 77a: ${ }^{1} \mathrm{H}$ NMR (400 MHz, $\left.\mathrm{CDCl}_{3}\right) \delta 8.04(\mathrm{~d}, J=8.4 \mathrm{~Hz}, 1 \mathrm{H}), 7.94(\mathrm{~d}, J=8.2$ Hz, 1H), 7.67 (t, $J=7.5 \mathrm{~Hz}, 1 \mathrm{H}), 7.50(\mathrm{t}, J=7.5 \mathrm{~Hz}, 1 \mathrm{H}), 7.15(\mathrm{~s}, 1 \mathrm{H}), 3.66(\mathrm{~s}, 3 \mathrm{H}), 2.96$ 
(t, $J=7.6 \mathrm{~Hz}, 2 \mathrm{H}), 2.67(\mathrm{~s}, 3 \mathrm{H}), 2.43(\mathrm{t}, J=7.5 \mathrm{~Hz}, 2 \mathrm{H}), 2.15(\mathrm{p}, J=7.6 \mathrm{~Hz}, 2 \mathrm{H}) ;{ }^{13} \mathrm{C}$ NMR (101 MHz, $\left.\mathrm{CDCl}_{3}\right) \delta$ 173.86, 161.22, 149.92, 144.92, 129.28, 128.99, 126.81, 125.70, 123.61, 122.04, 51.55, 37.88, 33.48, 24.89, 18.73; HRMS (ESI) m/z calculated for $\mathrm{C}_{15} \mathrm{H}_{18} \mathrm{O}_{2} \mathrm{~N}\left[(\mathrm{M}+\mathrm{H})^{+}\right]$244.1332, found 244.1330. IR (film) 2950, 1736, 1603, 1561, $1437,1247,1195,1173,760 \mathrm{~cm}^{-1}$.

Compound 77b: ${ }^{1} \mathrm{H}$ NMR (400 MHz, $\left.\mathrm{CDCl}_{3}\right) \delta 8.03(\mathrm{~d}, J=8.4 \mathrm{~Hz}, 1 \mathrm{H}), 7.94(\mathrm{~d}, J=8.3$ Hz, 1H), 7.66 (ddd, $J=8.4,6.8,1.4 \mathrm{~Hz}, 1 \mathrm{H}), 7.49$ (ddd, $J=8.2,6.8,1.3 \mathrm{~Hz}, 1 \mathrm{H}), 7.17$ (s, 1H), 3.63 (s, 3H), 3.56 (h, $J=7.2 \mathrm{~Hz}, 1 \mathrm{H}), 3.07$ (dd, $J=15.9,7.5 \mathrm{~Hz}, 1 \mathrm{H}), 2.69$ (dd, $J=$ 15.9, 7.2 Hz, 1H), $2.67(\mathrm{~s}, 3 \mathrm{H}), 1.40(\mathrm{~d}, J=7.0 \mathrm{~Hz}, 3 \mathrm{H}) ;{ }^{13} \mathrm{C} \mathrm{NMR}\left(101 \mathrm{MHz}, \mathrm{CDCl}_{3}\right) \delta$ $173.21,164.24,147.50,144.42,129.52,128.91,127.00,125.54,123.52,120.97,51.43$, 40.11, 38.44, 20.73, 18.72; HRMS (ESI) $\mathrm{m} / \mathrm{z}$ calculated for $\mathrm{C}_{15} \mathrm{H}_{18} \mathrm{O}_{2} \mathrm{~N}\left[(\mathrm{M}+\mathrm{H})^{+}\right]$ 244.1332, found 244.1330. IR (film) 2965, 1736, 1603, 1562, 1508, 1436, 1195, 1166 , $760 \mathrm{~cm}^{-1}$.

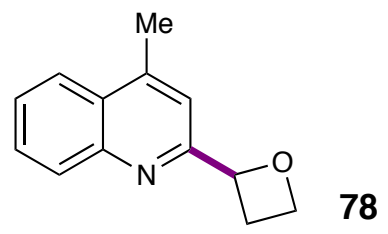

4-Methyl-2-(oxetan-2-yl)quinoline (78): According to the general procedure (Conditions A), lepidine (71.6 mg, $0.50 \mathrm{mmol}, 1.0$ equiv.), Selectfluor (373.0 mg, 1.0 mmol, 2.0 equiv.), hydrochloric acid ( $43.0 \mu 1,0.50 \mathrm{mmol}, 1.0$ equiv.), trimethylene oxide ( $0.65 \mathrm{ml}, 10.0 \mathrm{mmol}, 20.0$ equiv.), $5.0 \mathrm{~mL}$ of acetone were used. After 16 hours, the reaction mixture was subjected to the workup procedure outlined in the general procedure and purified by flash chromatography ( $20 \%$ ethyl acetate/hexanes) to provide the title compound as a colorless oil ( $64.7 \mathrm{mg}, 65 \%$ yield). 
${ }^{1} \mathrm{H}$ NMR (400 MHz, $\left.\mathrm{CDCl}_{3}\right) \delta 8.06(\mathrm{~d}, J=8.4 \mathrm{~Hz}, 1 \mathrm{H}), 7.98(\mathrm{~d}, J=8.3 \mathrm{~Hz}, 1 \mathrm{H}), 7.69$ (ddd, $J=8.3,6.9,1.5 \mathrm{~Hz}, 1 \mathrm{H}), 7.67$ (s, 1H), 7.53 (ddd, $J=8.3,6.8,1.3 \mathrm{~Hz}, 1 \mathrm{H}$ ), 5.99 (dd, $J=8.3,6.9 \mathrm{~Hz}, 1 \mathrm{H}), 4.92(\mathrm{td}, J=8.0,5.9 \mathrm{~Hz}, 1 \mathrm{H}), 4.77(\mathrm{dt}, J=9.1,6.0 \mathrm{~Hz}, 1 \mathrm{H}), 3.25-$ $3.15(\mathrm{~m}, 1 \mathrm{H}), 2.86-2.76(\mathrm{~m}, 1 \mathrm{H}), 2.74(\mathrm{~s}, 3 \mathrm{H}) ;{ }^{13} \mathrm{C} \mathrm{NMR}\left(101 \mathrm{MHz}, \mathrm{CDCl}_{3}\right) \delta 162.45$, $147.19,145.47,129.49,129.29,127.46,126.04,123.68,118.15,83.53,69.13,28.97$, 18.87; HRMS (ESI) $\mathrm{m} / \mathrm{z}$ calculated for $\mathrm{C}_{13} \mathrm{H}_{14} \mathrm{ON}\left[(\mathrm{M}+\mathrm{H})^{+}\right]$200.1070, found 200.1068; IR (film) 2964, 2884, 1601, 1561, 1508, 1448, 1037, 980, $760 \mathrm{~cm}^{-1}$.

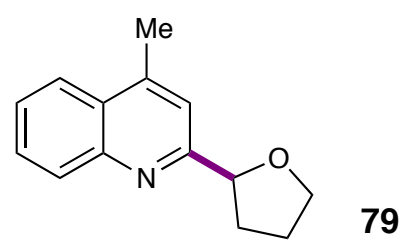

4-Methyl-2-(tetrahydrofuran-2-yl)quinoline (79): According to the general procedure (Conditions A), lepidine (71.6 mg, $0.50 \mathrm{mmol}, 1.0$ equiv.), Selectfluor (373.0 mg, 1.0 mmol, 2.0 equiv.), hydrochloric acid (43.0 $\mu 1,0.50 \mathrm{mmol}, 1.0$ equiv.), tetrahydrofuran (0.61 ml, $7.5 \mathrm{mmol}, 15.0$ equiv.), $5.0 \mathrm{~mL}$ of acetone were used. After 16 hours, the reaction mixture was subjected to the workup procedure outlined in the general procedure and purified by flash chromatography ( $20 \%$ ethyl acetate/hexanes) to provide the title compound as a colorless oil ( $83.1 \mathrm{mg}, 78 \%$ yield).

${ }^{1} \mathrm{H}$ NMR (400 MHz, $\left.\mathrm{CDCl}_{3}\right) \delta 8.06(\mathrm{~d}, J=8.4 \mathrm{~Hz}, 1 \mathrm{H}), 7.96(\mathrm{~d}, J=8.3 \mathrm{~Hz}, 1 \mathrm{H}), 7.68$ (ddd, $J=8.4,6.8,1.4 \mathrm{~Hz}, 1 \mathrm{H}), 7.52(\mathrm{ddd}, J=8.3,6.9,1.3 \mathrm{~Hz}, 1 \mathrm{H}), 7.44$ (s, $1 \mathrm{H}), 5.14(\mathrm{t}, J$ $=6.9 \mathrm{~Hz}, 1 \mathrm{H}), 4.21-4.13(\mathrm{~m}, 1 \mathrm{H}), 4.07-3.99(\mathrm{~m}, 1 \mathrm{H}), 2.70(\mathrm{~s}, 3 \mathrm{H}), 2.56-2.46(\mathrm{~m}$, 1H), $2.11-1.97(\mathrm{~m}, 3 \mathrm{H}) ;{ }^{13} \mathrm{C} \mathrm{NMR}\left(101 \mathrm{MHz}, \mathrm{CDCl}_{3}\right) \delta$ 163.01, 147.14, 145.06, 129.39, 129.15, 127.40, 125.82, 123.65, 118.54, 81.96, 69.23, 33.31, 25.93, 18.87; HRMS (ESI) $\mathrm{m} / \mathrm{z}$ calculated for $\mathrm{C}_{14} \mathrm{H}_{16} \mathrm{ON}\left[(\mathrm{M}+\mathrm{H})^{+}\right]$214.1226, found 214.1225; IR (film) 3046, 2976, 
$2872,1735,1602,1508,1448,1245,1070,760 \mathrm{~cm}^{-1}$. Spectra data are consistent with those reported in the literature: Synlett, 2016, 27, 1282-1286.

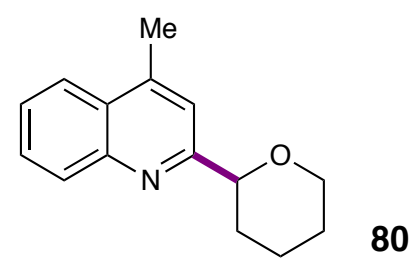

4-Methyl-2-(tetrahydro-2H-pyran-2-yl)quinoline (80): According to the general procedure (Conditions A), lepidine (71.6 mg, $0.50 \mathrm{mmol}, 1.0$ equiv.), Selectfluor (373.0 $\mathrm{mg}, 1.0 \mathrm{mmol}, 2.0$ equiv.), hydrochloric acid ( $43.0 \mu \mathrm{l}, 0.50 \mathrm{mmol}, 1.0$ equiv.), tetrahydro$2 H$-pyran ( $0.73 \mathrm{ml}, 7.5 \mathrm{mmol}, 15.0$ equiv.), $5.0 \mathrm{~mL}$ of acetone were used. After 16 hours, the reaction mixture was subjected to the workup procedure outlined in the general procedure and purified by flash chromatography ( $20 \%$ ethyl acetate/hexanes) to provide the title compound as a colorless oil ( $97.8 \mathrm{mg}, 86 \%$ yield).

${ }^{1} \mathrm{H}$ NMR $\left(400 \mathrm{MHz}, \mathrm{CDCl}_{3}\right) \delta 8.07(\mathrm{~d}, J=8.4 \mathrm{~Hz}, 1 \mathrm{H}), 7.96(\mathrm{~d}, J=8.3 \mathrm{~Hz}, 1 \mathrm{H}), 7.67(\mathrm{t}$, $J=7.6 \mathrm{~Hz}, 1 \mathrm{H}), 7.51(\mathrm{t}, J=7.6 \mathrm{~Hz}, 1 \mathrm{H}), 7.46(\mathrm{~s}, 1 \mathrm{H}), 4.61(\mathrm{dd}, J=11.1,2.3 \mathrm{~Hz}, 1 \mathrm{H})$, $4.21(\mathrm{dd}, J=11.4,4.1 \mathrm{~Hz}, 1 \mathrm{H}), 3.69$ (td, $J=11.6,2.5 \mathrm{~Hz}, 1 \mathrm{H}), 2.70$ (s, 3H), $2.15-2.06$ (m, 1H), $2.03-1.93(\mathrm{~m}, 1 \mathrm{H}), 1.82-1.69(\mathrm{~m}, 2 \mathrm{H}), 1.69-1.56(\mathrm{~m}, 2 \mathrm{H}) ;{ }^{13} \mathrm{C}$ NMR (101 $\left.\mathrm{MHz}, \mathrm{CDCl}_{3}\right) \delta 162.07,147.02,145.06,129.51,129.06,127.48,125.82,123.60,118.76$, 81.54, 68.86, 32.75, 25.82, 23.68, 18.80; HRMS (ESI) m/z calculated for $\mathrm{C}_{15} \mathrm{H}_{18} \mathrm{NO}$ $\left[(\mathrm{M}+\mathrm{H})^{+}\right]$228.1383, found 228.1383. IR (film) 2936, 2848, 1603, 1507, 1448, 1205, $1089,1046,758 \mathrm{~cm}^{-1}$. Spectra data are consistent with those reported in the literature: Angew. Chem. Int. Ed. 2015, 54, 1565-1569. 


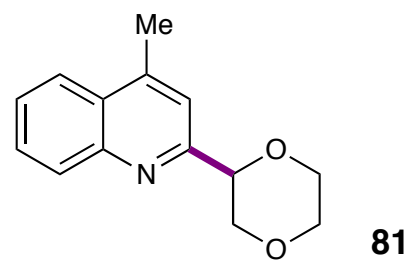

2-(1,4-Dioxan-2-yl)-4-methylquinoline (81): According to the general procedure (Conditions A), lepidine (71.6 mg, $0.50 \mathrm{mmol}, 1.0$ equiv.), Selectfluor (373.0 mg, 1.0 mmol, 2.0 equiv.), hydrochloric acid (43.0 $\mu 1,0.50 \mathrm{mmol}, 1.0$ equiv.), 1,4-dioxane (0.64 $\mathrm{ml}, 7.5 \mathrm{mmol}, 15.0$ equiv.), $5.0 \mathrm{~mL}$ of acetone were used. After 16 hours, the reaction mixture was subjected to the workup procedure outlined in the general procedure and purified by flash chromatography (20\% ethyl acetate/hexanes) to provide the title compound as a white solid ( $96.3 \mathrm{mg}, 84 \%$ yield).

${ }^{1} \mathrm{H}$ NMR (400 MHz, $\left.\mathrm{CDCl}_{3}\right) \delta 8.07(\mathrm{~d}, J=8.4 \mathrm{~Hz}, 1 \mathrm{H}), 7.97(\mathrm{~d}, J=8.3 \mathrm{~Hz}, 1 \mathrm{H}), 7.72-$

$7.65(\mathrm{~m}, 1 \mathrm{H}), 7.57-7.50(\mathrm{~m}, 1 \mathrm{H}), 7.45(\mathrm{~s}, 1 \mathrm{H}), 4.89$ (dd, $J=10.1,2.9 \mathrm{~Hz}, 1 \mathrm{H}), 4.23(\mathrm{dd}$, $J=11.6,2.9 \mathrm{~Hz}, 1 \mathrm{H}), 4.05-3.94(\mathrm{~m}, 2 \mathrm{H}), 3.87-3.74(\mathrm{~m}, 2 \mathrm{H}), 3.63(\mathrm{dd}, J=11.6,10.1$ $\mathrm{Hz}, 1 \mathrm{H}), 2.71(\mathrm{~s}, 3 \mathrm{H}) ;{ }^{13} \mathrm{C} \mathrm{NMR}\left(101 \mathrm{MHz}, \mathrm{CDCl}_{3}\right) \delta 157.72,147.13,145.23,129.66$, 129.26, 127.55, 126.18, 123.63, 119.04, 78.67, 71.03, 67.00, 66.35, 18.81; HRMS (ESI) $\mathrm{m} / \mathrm{z}$ calculated for $\mathrm{C}_{14} \mathrm{H}_{16} \mathrm{NO}_{2}\left[(\mathrm{M}+\mathrm{H})^{+}\right] 230.1175$, found 230.1175; IR (film) 2980, 2849, $1600,1506,1128,1111,913,866,759 \mathrm{~cm}^{-1}$. Spectra data are consistent with those reported in the literature: Synlett, 2016, 27, 1282-1286.

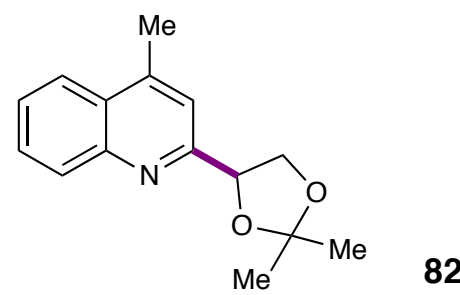

2-(2,2-Dimethyl-1,3-dioxolan-4-yl)-4-methylquinoline (82): According to the general procedure (Conditions B), lepidine (71.6 mg, $0.50 \mathrm{mmol}, 1.0$ equiv.), Selectfluor (373.0 
$\mathrm{mg}, 1.0 \mathrm{mmol}, 2.0$ equiv.), trifluoroacetic acid (72.3 $\mu 1,1.0 \mathrm{mmol}, 2.0$ equiv.), 2,2dimethyl-1,3-dioxolane ( $1.1 \mathrm{ml}, 10.0 \mathrm{mmol}, 20.0$ equiv.), $5.0 \mathrm{~mL}$ of acetonitrile were used. After 16 hours, the reaction mixture was subjected to the workup procedure outlined in the general procedure and purified by flash chromatography (10\% ethyl acetate/hexanes) to provide the title compound as a colorless oil (94.9 mg, 78\% yield). ${ }^{1} \mathrm{H}$ NMR (400 MHz, $\left.\mathrm{CDCl}_{3}\right) \delta 8.03(\mathrm{~d}, J=8.4 \mathrm{~Hz}, 1 \mathrm{H}), 7.97(\mathrm{~d}, J=8.3 \mathrm{~Hz}, 1 \mathrm{H}), 7.68$ (ddd, $J=8.4,6.8,1.4 \mathrm{~Hz}, 1 \mathrm{H}), 7.53(\mathrm{ddd}, J=8.2,6.8,1.3 \mathrm{~Hz}, 1 \mathrm{H}), 7.51$ (s, $1 \mathrm{H}), 5.34(\mathrm{t}, J$ $=6.9 \mathrm{~Hz}, 1 \mathrm{H}), 4.53(\mathrm{dd}, J=8.4,6.9 \mathrm{~Hz}, 1 \mathrm{H}), 4.04(\mathrm{dd}, J=8.4,6.9 \mathrm{~Hz}, 1 \mathrm{H}), 2.71(\mathrm{~s}, 3 \mathrm{H})$, $1.59(\mathrm{~s}, 3 \mathrm{H}), 1.52(\mathrm{~s}, 3 \mathrm{H}) ;{ }^{13} \mathrm{C} \mathrm{NMR}\left(101 \mathrm{MHz}, \mathrm{CDCl}_{3}\right) \delta 159.85,147.05,145.43$, $129.37,129.33,127.57,126.14,123.70,118.51,110.32,78.73,70.21,26.44,25.48$, 18.90; HRMS (ESI) $\mathrm{m} / \mathrm{z}$ calculated for $\mathrm{C}_{15} \mathrm{H}_{18} \mathrm{O}_{2} \mathrm{~N}\left[(\mathrm{M}+\mathrm{H})^{+}\right]$244.1332, found 244.1330. IR (film) 2986, 2934, 1714, 1602, 1563, 1509, 1451, 1381, 1371, 1218, 1155, 1065, 847, $760 \mathrm{~cm}^{-1}$.<smiles>O=[N+]([O-])c1cc(C2OCOCO2)nc2ccccc12</smiles>

4-Methyl-2-(1,3,5-trioxan-2-yl)quinoline (83): According to the general procedure (Conditions A), lepidine (71.6 mg, $0.50 \mathrm{mmol}, 1.0$ equiv.), Selectfluor (373.0 mg, 1.0 mmol, 2.0 equiv.), hydrochloric acid ( $43.0 \mu 1,0.50 \mathrm{mmol}, 1.0$ equiv.), 1,3,5-trioxane (675 $\mathrm{mg}, 7.5 \mathrm{mmol}, 15.0$ equiv.), $5.0 \mathrm{~mL}$ of acetone were used. After 16 hours, the reaction mixture was subjected to the workup procedure outlined in the general procedure and purified by flash chromatography (20\% ethyl acetate/hexanes) to provide the title compound as a white solid ( $54.3 \mathrm{mg}, 47 \%$ yield). 
${ }^{1} \mathrm{H}$ NMR (400 MHz, $\left.\mathrm{CDCl}_{3}\right) \delta 8.14(\mathrm{~d}, J=8.4 \mathrm{~Hz}, 1 \mathrm{H}), 7.98(\mathrm{~d}, J=8.9 \mathrm{~Hz}, 1 \mathrm{H}), 7.71$ (ddd, $J=8.4,6.8,1.4 \mathrm{~Hz}, 1 \mathrm{H}), 7.64(\mathrm{~s}, 1 \mathrm{H}), 7.57(\mathrm{ddd}, J=8.2,6.8,1.3 \mathrm{~Hz}, 1 \mathrm{H}), 6.08(\mathrm{~s}$, 1H), $5.40(\mathrm{q}, J=6.3 \mathrm{~Hz}, 4 \mathrm{H}), 2.72(\mathrm{~s}, 3 \mathrm{H}) ;{ }^{13} \mathrm{C} \mathrm{NMR}\left(101 \mathrm{MHz}, \mathrm{CDCl}_{3}\right) \delta 154.72$, $146.92,145.93,130.03,129.43,128.30,126.91,123.66,118.80,102.05,93.63,18.82$; HRMS (ESI) $\mathrm{m} / \mathrm{z}$ calculated for $\mathrm{C}_{13} \mathrm{H}_{14} \mathrm{O}_{3} \mathrm{~N}\left[(\mathrm{M}+\mathrm{H})^{+}\right]$232.0968, found 232.0966. IR (film) 2877, 1605, 1572, 1418, 1393, 1183, 1154, 1084, 1009, 940, $758 \mathrm{~cm}^{-1}$. Spectra data are consistent with those reported in the literature: Bioorg. Med. Chem. Lett. 2012, $22,3480-3484$.

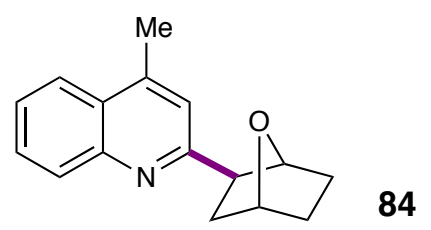

2-(7-Oxabicyclo[2.2.1]heptan-2-yl)-4-methylquinoline (84): According to the general procedure (Conditions B), lepidine (71.6 mg, $0.50 \mathrm{mmol}, 1.0$ equiv.), Selectfluor (373.0 mg, 1.0 mmol, 2.0 equiv.), trifluoroacetic acid (72.3 $\mu \mathrm{l}, 1.0 \mathrm{mmol}, 2.0$ equiv.), 7oxabicyclo[2.2.1]heptane (1.0 ml, $10.0 \mathrm{mmol}, 20.0$ equiv.), $5.0 \mathrm{~mL}$ of acetonitrile were used. After 16 hours, the reaction mixture was subjected to the workup procedure outlined in the general procedure and purified by flash chromatography $(20 \%$ ethyl acetate/hexanes) to provide the title compound as a colorless oil (107.7 mg, 90\% yield). ${ }^{1} \mathrm{H}$ NMR (400 MHz, $\left.\mathrm{CDCl}_{3}\right) \delta 8.03(\mathrm{~d}, J=8.4 \mathrm{~Hz}, 1 \mathrm{H}), 7.93(\mathrm{~d}, J=8.3 \mathrm{~Hz}, 1 \mathrm{H}), 7.66$ (ddd, $J=8.4,6.9,1.5 \mathrm{~Hz}, 1 \mathrm{H}), 7.50$ (ddd, $J=8.2,6.8,1.3 \mathrm{~Hz}, 1 \mathrm{H}), 7.42$ (s, $1 \mathrm{H}), 4.79$ (t, $J$ $=4.8 \mathrm{~Hz}, 1 \mathrm{H}), 4.70(\mathrm{~d}, J=4.2 \mathrm{~Hz}, 1 \mathrm{H}), 3.34(\mathrm{dd}, J=9.1,5.1 \mathrm{~Hz}, 1 \mathrm{H}), 2.68(\mathrm{~s}, 3 \mathrm{H}), 2.17$ (dd, $J=12.3,9.1 \mathrm{~Hz}, 1 \mathrm{H}), 2.04-1.95(\mathrm{~m}, 1 \mathrm{H}), 1.88-1.77(\mathrm{~m}, 2 \mathrm{H}), 1.76-1.67(\mathrm{~m}, 1 \mathrm{H})$, $1.64-1.55(\mathrm{~m}, 1 \mathrm{H}) ;{ }^{13} \mathrm{C} \mathrm{NMR}\left(101 \mathrm{MHz}, \mathrm{CDCl}_{3}\right) \delta 164.77,146.50,145.37,129.19$, $129.09,127.04,125.68,123.54,120.08,81.52,76.45,51.72,39.99,30.45,29.65,18.85$; 
HRMS (ESI) $\mathrm{m} / \mathrm{z}$ calculated for $\mathrm{C}_{16} \mathrm{H}_{18} \mathrm{ON}\left[(\mathrm{M}+\mathrm{H})^{+}\right] 240.1383$, found 240.1381. IR (film) 2976, 1604, 1560, 1507, 1447, 1347, 1202, 1000, $760 \mathrm{~cm}^{-1}$.
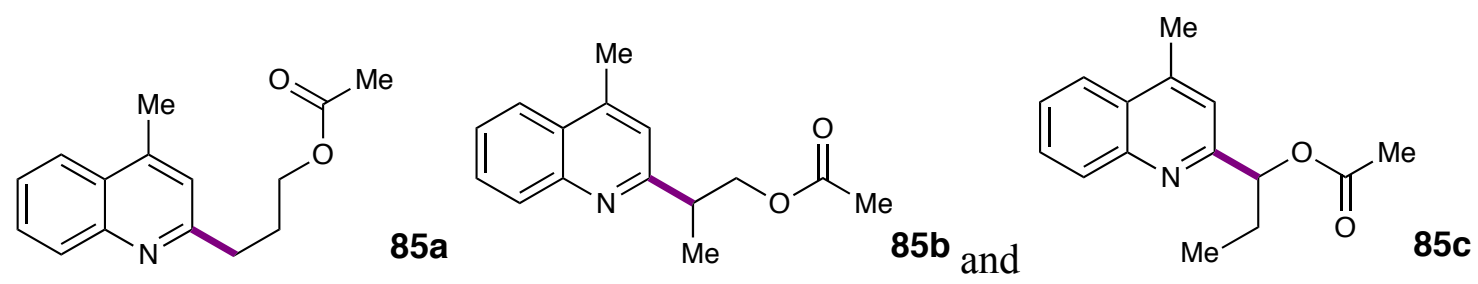

3-(4-Methylquinolin-2-yl)propyl acetate (85a), 2-(4-Methylquinolin-2-yl)propyl acetate (85b) and 1-(4-methylquinolin-2-yl)propyl acetate (85c): According to the general procedure (Conditions A), lepidine ( $71.6 \mathrm{mg}, 0.50 \mathrm{mmol}, 1.0$ equiv.), Selectfluor (466.3 mg, $1.25 \mathrm{mmol}, 2.5$ equiv.), hydrochloric acid (43.0 $\mu \mathrm{l}, 0.50 \mathrm{mmol}, 1.0$ equiv.), propyl acetate ( $1.20 \mathrm{ml}, 10.0 \mathrm{mmol}, 20.0$ equiv.), $5.0 \mathrm{~mL}$ of acetone were used. After 24 hours, the reaction mixture was subjected to the workup procedure outlined in the general procedure and purified by flash chromatography (5\% ethyl acetate/hexanes) to provide the title compounds as a colorless oil (15.8 $\mathrm{mg}, 13 \%$ yield for $\mathbf{8 5 a}$ ) and a colorless oil mixture of $47.4 \mathrm{mg}$ ( $26 \%$ yield for $\mathbf{8 5 b} ; 13 \%$ yield for $\mathbf{8 5 c}$ ).

Compounds 85a: ${ }^{1} \mathrm{H}$ NMR $\left(400 \mathrm{MHz}, \mathrm{CDCl}_{3}\right) \delta 8.08(\mathrm{~d}, J=8.3 \mathrm{~Hz}, 1 \mathrm{H}), 7.96(\mathrm{~d}, J=7.8$ Hz, 1H), 7.69 (ddd, $J=8.4,6.8,1.4 \mathrm{~Hz}, 1 \mathrm{H}), 7.53$ (ddd, $J=8.2,6.8,1.2 \mathrm{~Hz}, 1 \mathrm{H}), 7.16$ (s, 1H), $4.18(\mathrm{t}, J=6.5 \mathrm{~Hz}, 2 \mathrm{H}), 3.03(\mathrm{t}, J=7.8 \mathrm{~Hz}, 2 \mathrm{H}), 2.69(\mathrm{~s}, 3 \mathrm{H}), 2.23-2.13(\mathrm{~m}, 2 \mathrm{H})$, 2.04 (s, 3H); ${ }^{13} \mathrm{C}$ NMR $\left(101 \mathrm{MHz}, \mathrm{CDCl}_{3}\right) \delta 171.17,160.94,147.13,145.19,129.45$, $128.90,126.82,125.84,123.63,122.03,64.00,35.05,28.53,20.96,18.76$; HRMS (ESI) $\mathrm{m} / \mathrm{z}$ calculated for $\mathrm{C}_{15} \mathrm{H}_{18} \mathrm{O}_{2} \mathrm{~N}\left[(\mathrm{M}+\mathrm{H})^{+}\right]$244.1332, found 244.1330; IR (film) 2957, $1737,1603,1561,1508,1448,1365,1244,1041,761 \mathrm{~cm}^{-1}$. Compounds 85b and 85c: HRMS (ESI) $\mathrm{m} / \mathrm{z}$ calculated for $\mathrm{C}_{15} \mathrm{H}_{18} \mathrm{O}_{2} \mathrm{~N}\left[(\mathrm{M}+\mathrm{H})^{+}\right]$ 244.1332, found 244.1330; IR (film) 2972, 1740, 1600, 1554, 1508, 1446, 1372, 1237, 
$762 \mathrm{~cm}^{-1}$. Compounds 85b: ${ }^{1} \mathrm{H}$ NMR $\left(400 \mathrm{MHz}, \mathrm{CDCl}_{3}\right) \delta 8.05(\mathrm{~d}, J=8.4 \mathrm{~Hz}, 1 \mathrm{H}), 7.93$ (d, $J=8.3 \mathrm{~Hz}, 1 \mathrm{H}), 7.70-7.62(\mathrm{~m}, 1 \mathrm{H}), 7.55-7.46(\mathrm{~m}, 1 \mathrm{H}), 7.14(\mathrm{~s}, 1 \mathrm{H}), 4.49$ (dd, $J=$ 10.9, 7.5 Hz, 1H), 4.37 (dd, $J=10.8,6.8 \mathrm{~Hz}, 1 \mathrm{H}), 3.40$ (h, $J=7.1 \mathrm{~Hz}, 1 \mathrm{H}), 2.66(\mathrm{~s}, 3 \mathrm{H})$, $1.97(\mathrm{~s}, 3 \mathrm{H}), 1.39(\mathrm{~d}, J=7.0 \mathrm{~Hz}, 3 \mathrm{H}) ;{ }^{13} \mathrm{C} \mathrm{NMR}\left(101 \mathrm{MHz}, \mathrm{CDCl}_{3}\right) \delta 170.99,162.42$, $147.47,144.55,129.43,129.01,127.02,125.67,123.49,120.64,68.17,41.45,20.80$, 18.67, 17.19. Compounds 85c: ${ }^{1} \mathrm{H}$ NMR (400 MHz, $\left.\mathrm{CDCl}_{3}\right) \delta 8.09(\mathrm{~d}, J=8.5 \mathrm{~Hz}, 1 \mathrm{H})$, $7.93(\mathrm{~d}, J=8.3 \mathrm{~Hz}, 1 \mathrm{H}), 7.70-7.62(\mathrm{~m}, 1 \mathrm{H}), 7.55-7.46(\mathrm{~m}, 1 \mathrm{H}), 7.26(\mathrm{~s}, 1 \mathrm{H}), 5.83(\mathrm{t}, J$ $=6.8 \mathrm{~Hz}, 1 \mathrm{H}), 2.68(\mathrm{~s}, 3 \mathrm{H}), 2.15(\mathrm{~s}, 3 \mathrm{H}), 2.04(\mathrm{p}, J=7.3 \mathrm{~Hz}, 2 \mathrm{H}), 0.97$ (t, $J=7.4 \mathrm{~Hz}$, 3H); ${ }^{13} \mathrm{C}$ NMR (101 MHz, $\left.\mathrm{CDCl}_{3}\right) \delta 170.43,159.47,147.25,144.94,129.70,129.16$, $127.42,126.07,123.50,119.22,78.42,28.04,21.07,18.79,9.80$.

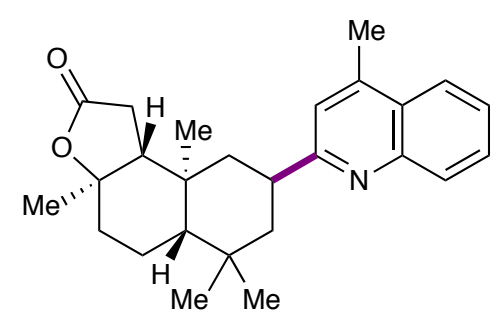

86

\section{(3aR,5aS,8S,9aS,9bR)-3a,6,6,9a-tetramethyl-8-(4-methylquinolin-2-}

yl)decahydronaphtho[2,1-b]furan-2(1H)-one (86): According to the general procedure (Conditions A), lepidine (71.6 mg, $0.50 \mathrm{mmol}, 1.0$ equiv.), Selectfluor (466.3 mg, 1.25 mmol, 2.5 equiv.), hydrochloric acid ( $43.0 \mu 1,0.50 \mathrm{mmol}, 1.0$ equiv.), sclareolide (1.252 $\mathrm{g}, 5.0 \mathrm{mmol}, 10.0$ equiv.), $10.0 \mathrm{~mL}$ of acetone were used. After 16 hours, the reaction mixture was subjected to the workup procedure outlined in the general procedure and purified by flash chromatography (50\% ethyl acetate/hexanes) to provide the title compound as a white solid (109.6 mg, 56\% yield).

${ }^{1} \mathrm{H}$ NMR (400 MHz, $\left.\mathrm{CDCl}_{3}\right) \delta 8.06(\mathrm{~d}, J=8.4 \mathrm{~Hz}, 1 \mathrm{H}), 7.94(\mathrm{~d}, J=8.2 \mathrm{~Hz}, 1 \mathrm{H}), 7.67(\mathrm{t}$, $J=7.3 \mathrm{~Hz}, 1 \mathrm{H}), 7.50(\mathrm{t}, J=7.5 \mathrm{~Hz}, 1 \mathrm{H}), 7.16(\mathrm{~s}, 1 \mathrm{H}), 3.41-3.24(\mathrm{~m}, 1 \mathrm{H}), 2.69(\mathrm{~s}, 3 \mathrm{H})$, 
$2.41(\mathrm{dd}, J=16.2,14.7 \mathrm{~Hz}, 1 \mathrm{H}), 2.24(\mathrm{dd}, J=16.2,6.4 \mathrm{~Hz}, 1 \mathrm{H}), 2.16-2.05(\mathrm{~m}, 2 \mathrm{H})$, $2.00-1.91(\mathrm{~m}, 1 \mathrm{H}), 1.79-1.68(\mathrm{t}, J=14.2 \mathrm{~Hz}, 3 \mathrm{H}), 1.67-1.60(\mathrm{~m}, 1 \mathrm{H}), 1.53-1.42$ $(\mathrm{m}, 2 \mathrm{H}), 1.36(\mathrm{~s}, 3 \mathrm{H}), 1.30-1.22(\mathrm{~m}, 1 \mathrm{H}), 1.10(\mathrm{~s}, 3 \mathrm{H}), 1.00(\mathrm{~s}, 3 \mathrm{H}), 0.98(\mathrm{~s}, 3 \mathrm{H}) ;{ }^{13} \mathrm{C}$ NMR $\left(101 \mathrm{MHz}, \mathrm{CDCl}_{3}\right) \delta 176.59,164.90,147.33,144.80,129.17,129.15,127.03$, $125.66,123.56,120.51,86.21,58.96,56.24,48.01,45.20,38.65,38.62,36.69,33.92$, $33.09,28.64,21.59,21.39,20.42,18.80,15.74 ;$ HRMS (ESI) $\mathrm{m} / \mathrm{z}$ calculated for $\mathrm{C}_{26} \mathrm{H}_{34} \mathrm{O}_{2} \mathrm{~N}\left[(\mathrm{M}+\mathrm{H})^{+}\right]$392.2584, found 392.2583. IR (film) 2956, 2927, 2851, 1712, $1503,1297,1211,1088,1019,764 \mathrm{~cm}^{-1}$. 


\section{NMR Spectra}
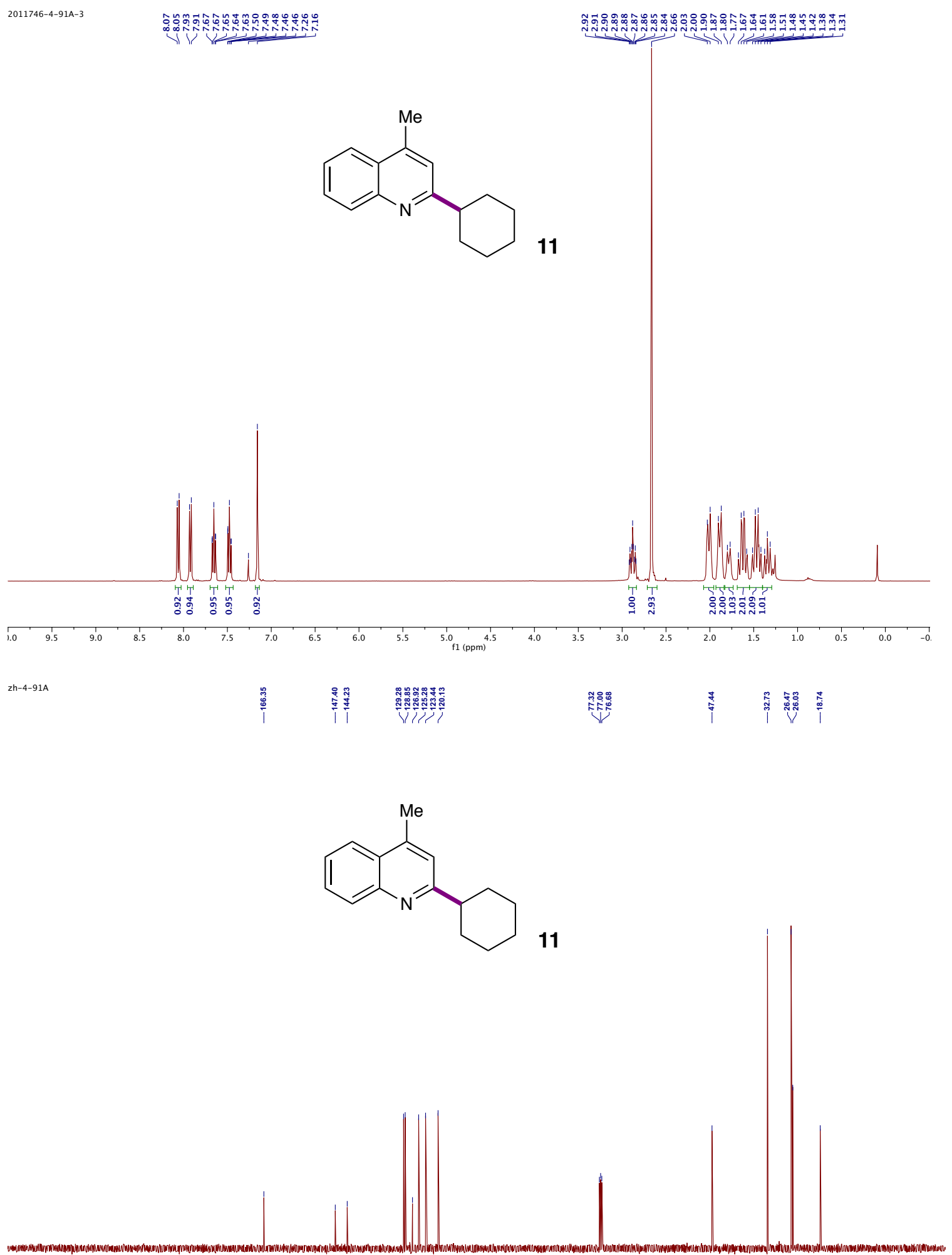

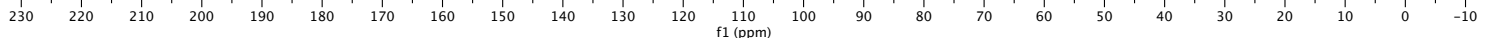




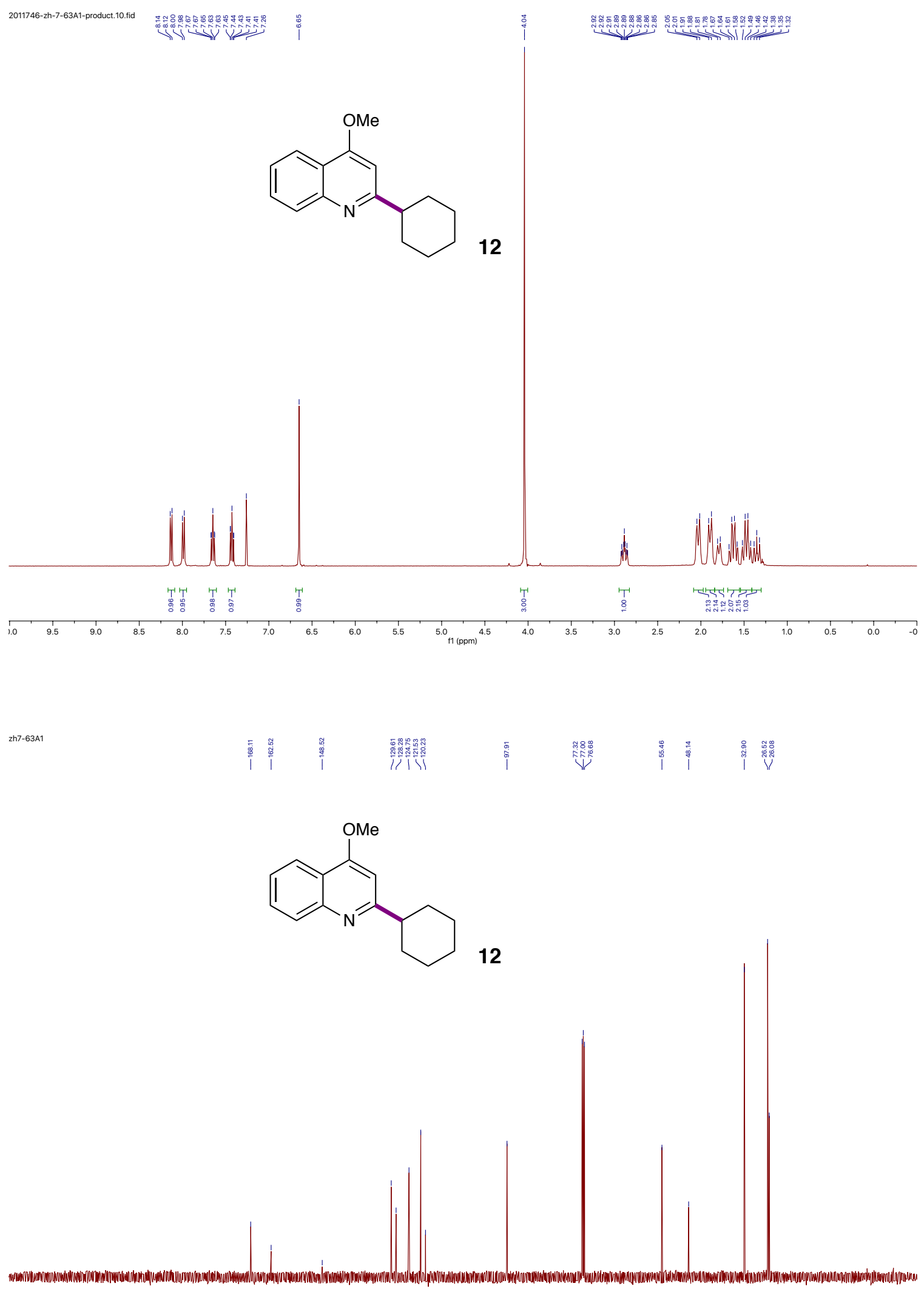

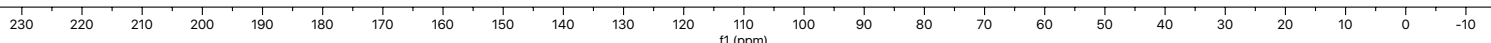




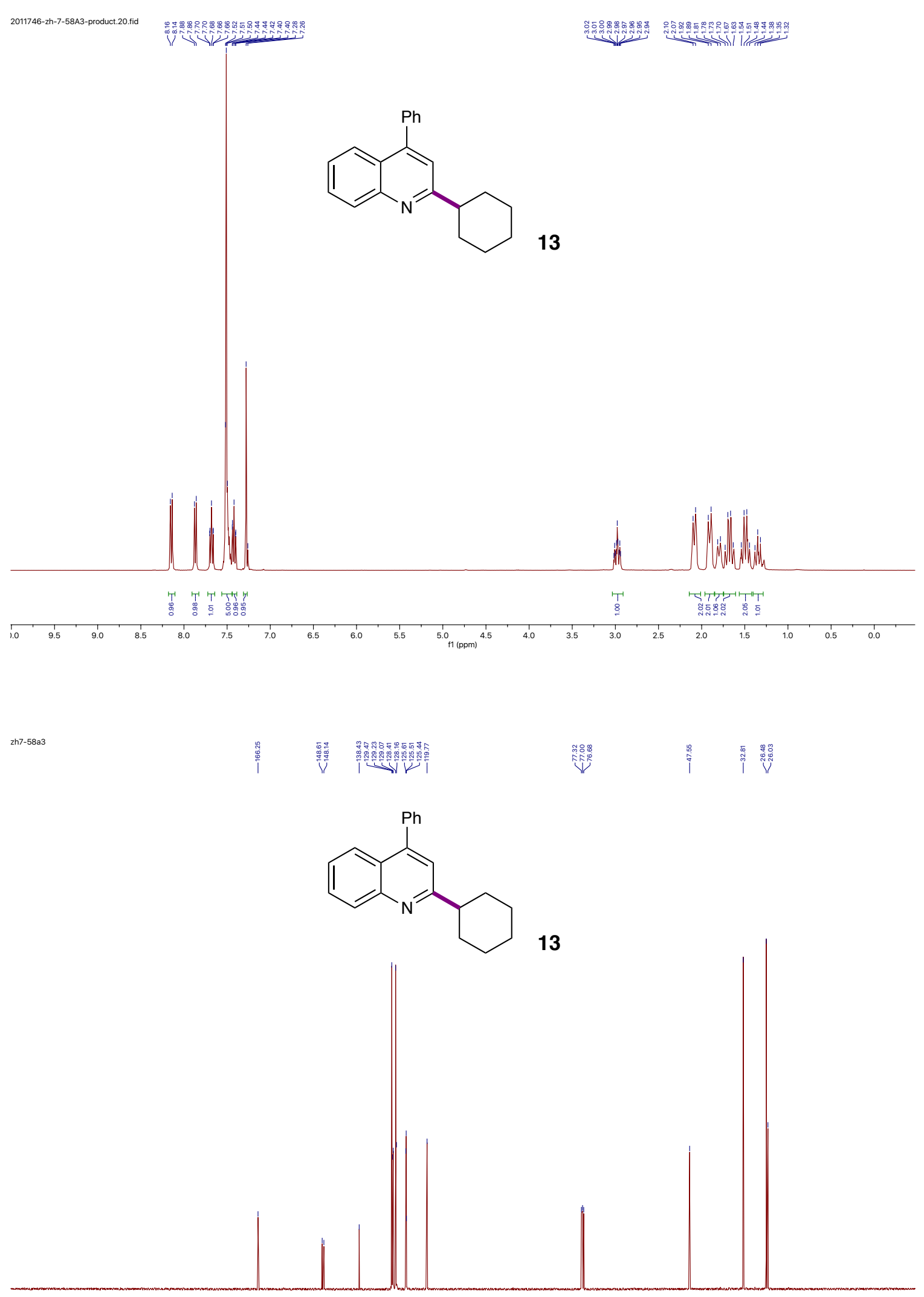

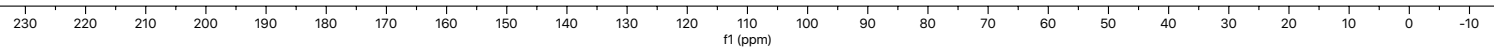




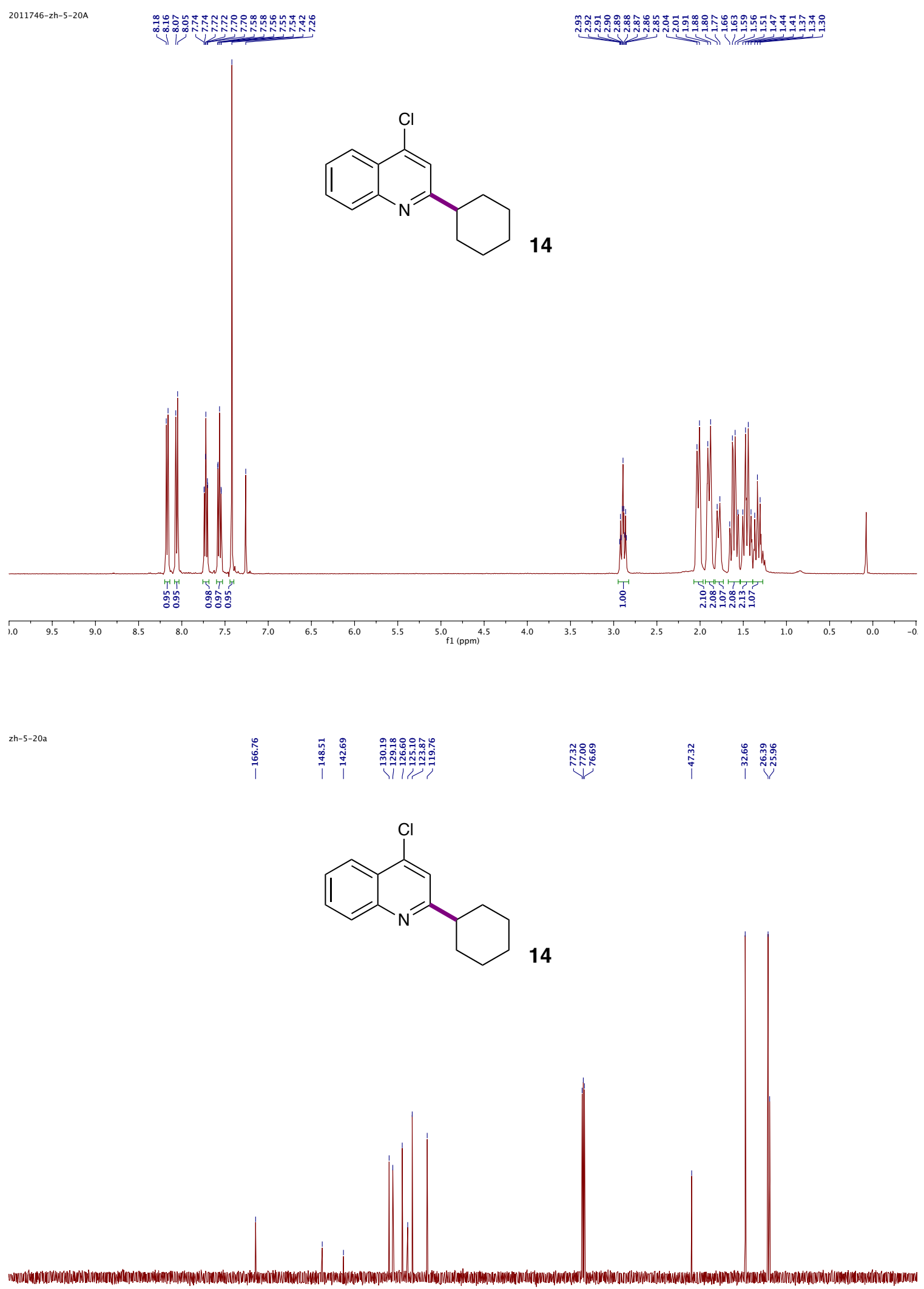

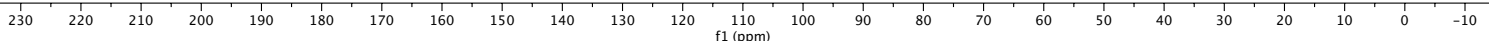


2011746-2h-9-26C1-part110.idido<smiles>[X]c1cc(C2CCCCC2)c2ccccc2n1</smiles>

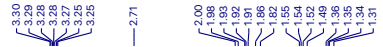

15

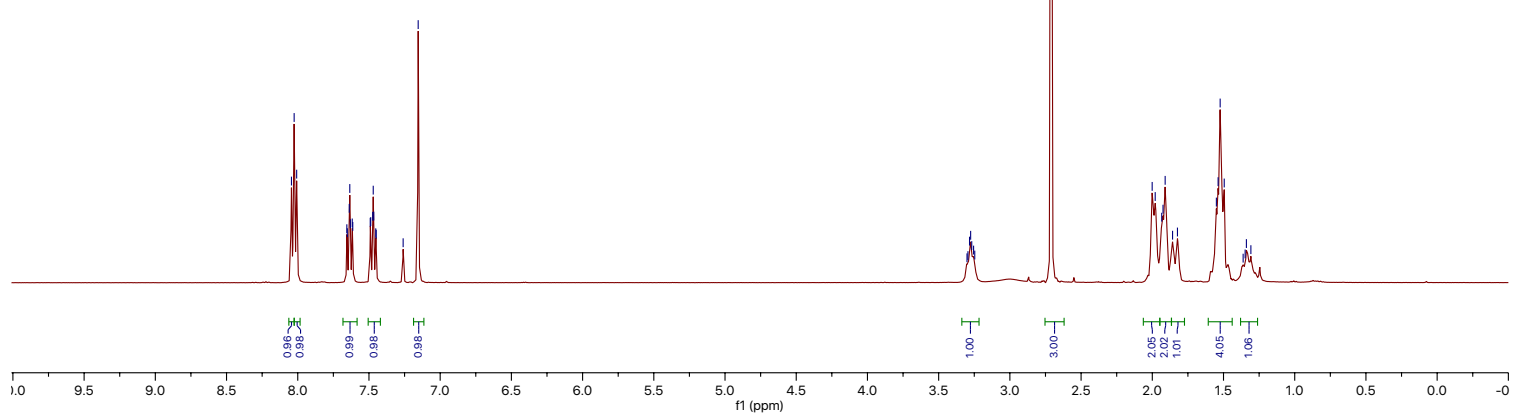

$-2 h-9-26 \mathrm{Cl}$<smiles>Cc1cc(C2CCCCC2)c2ccccc2n1</smiles>

15

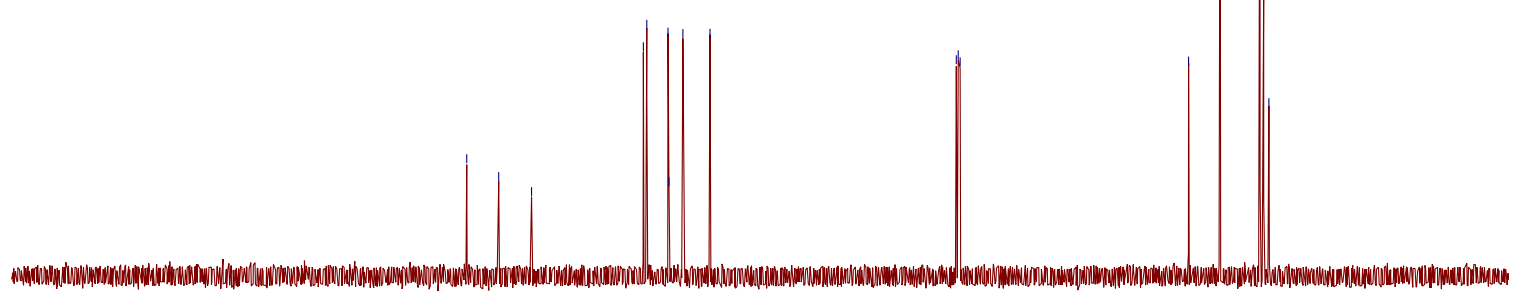

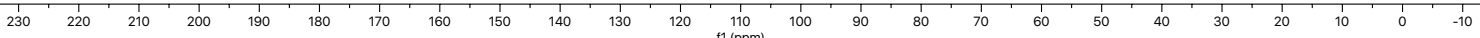




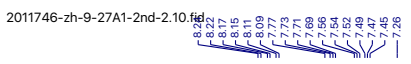

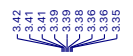

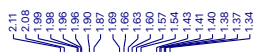
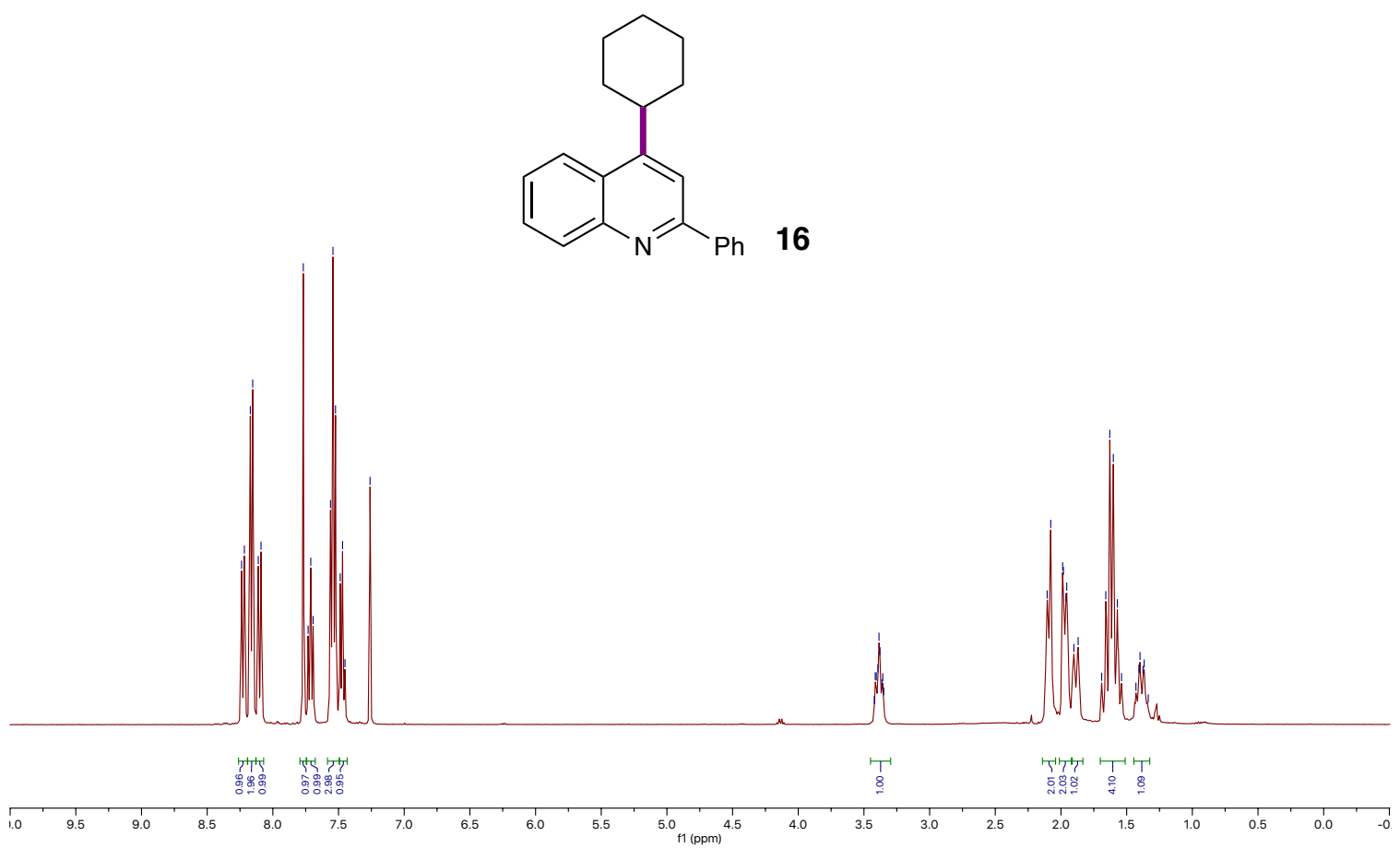

-zh-9-27A1
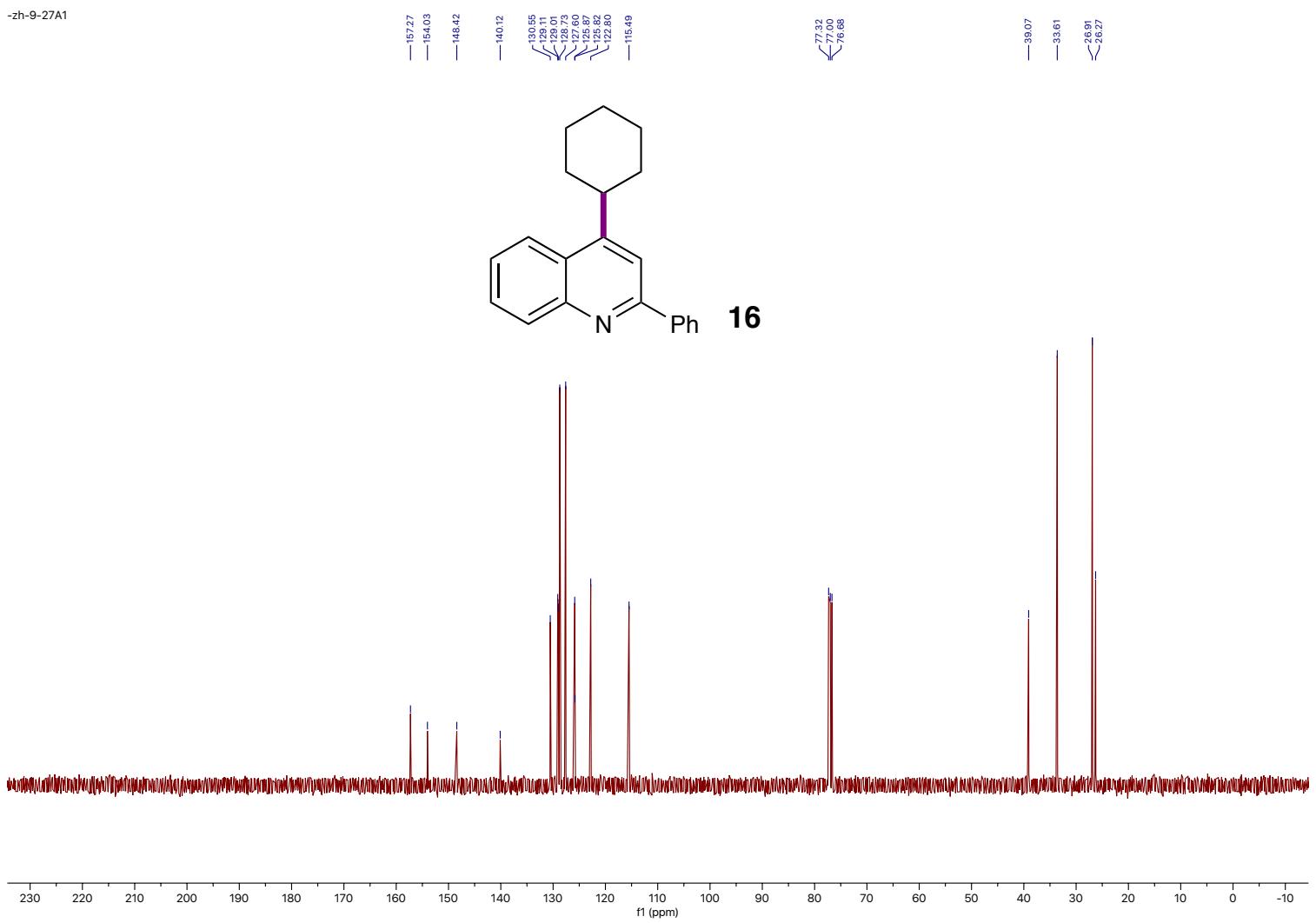

81 


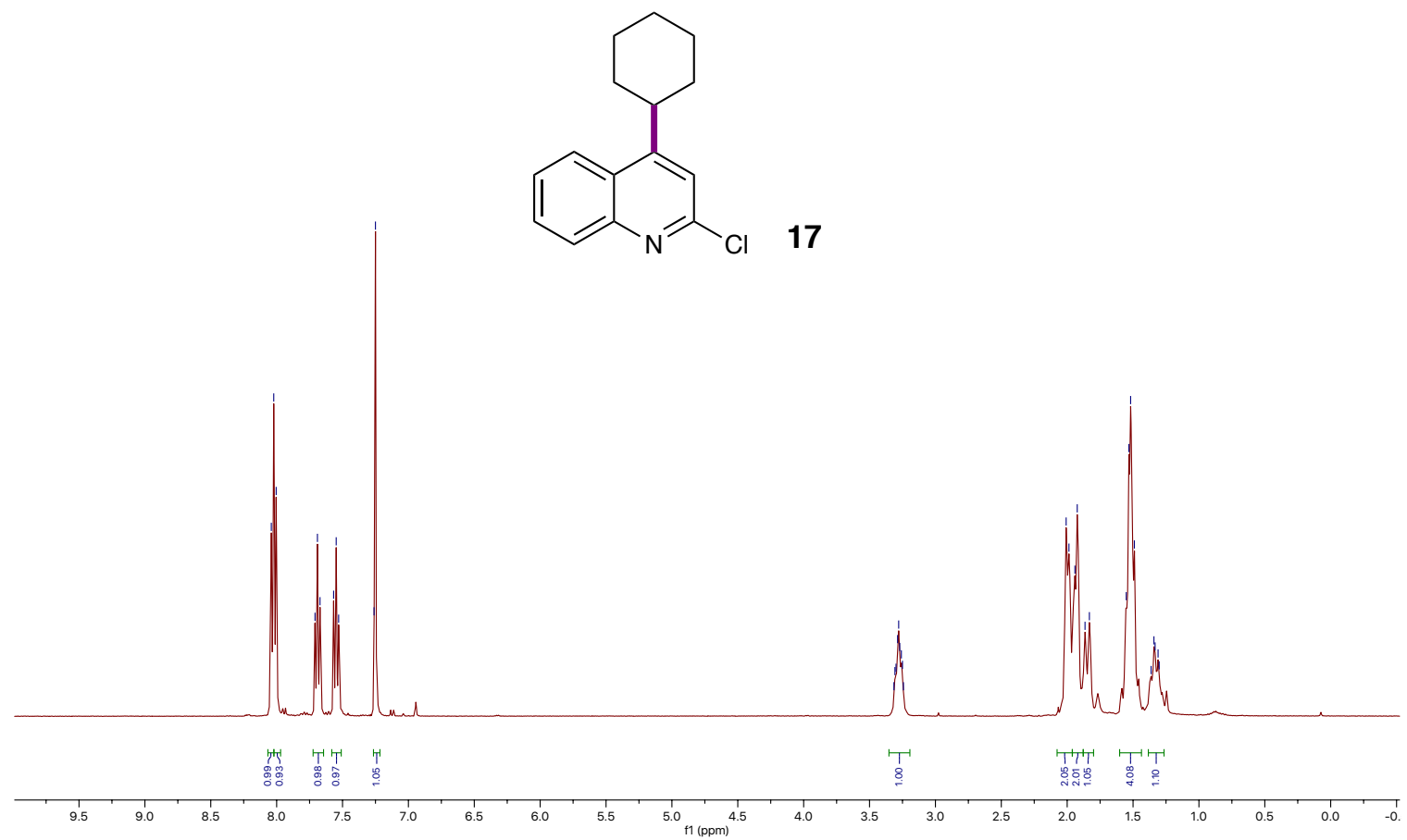

zh-9-31-part1<smiles>Clc1cc(C2CCCCC2)c2ccccc2n1</smiles>

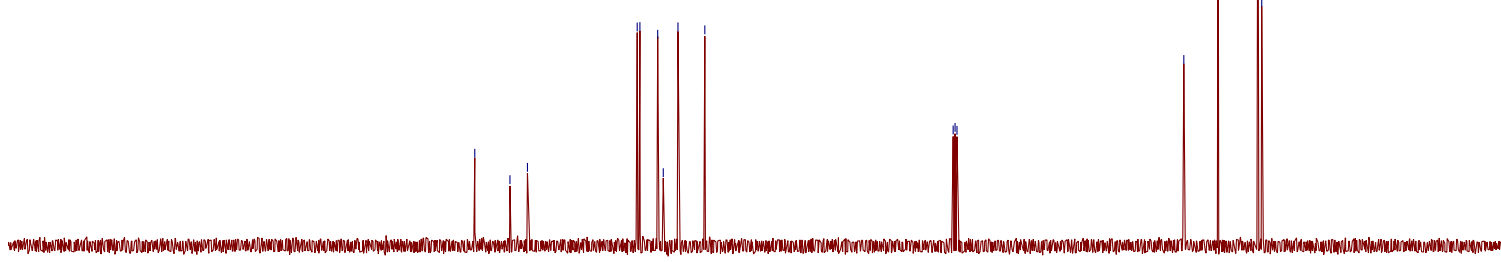

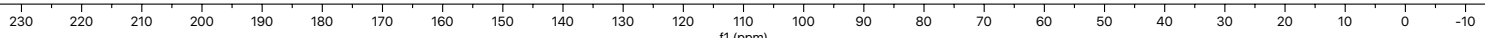


<smiles>c1ccc2c(C3CCCCC3)nccc2c1</smiles>

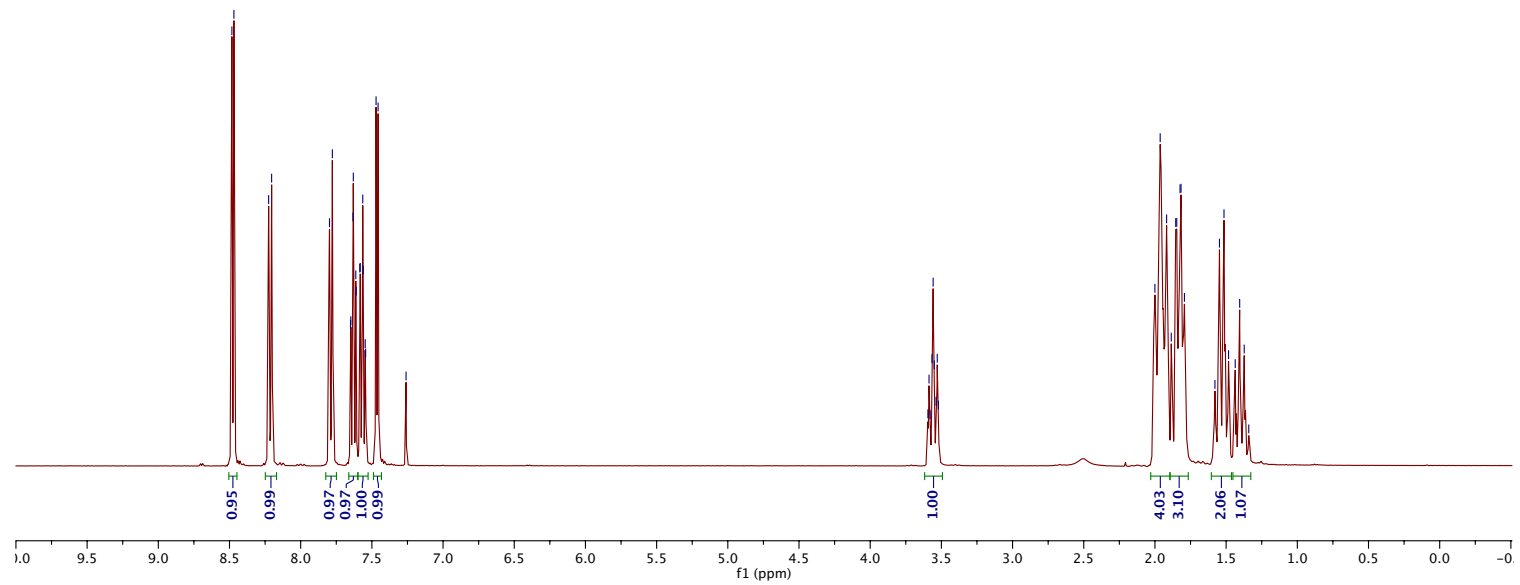

zh-5-77cC<smiles>c1ccc2c(C3CCCCC3)nccc2c1</smiles>

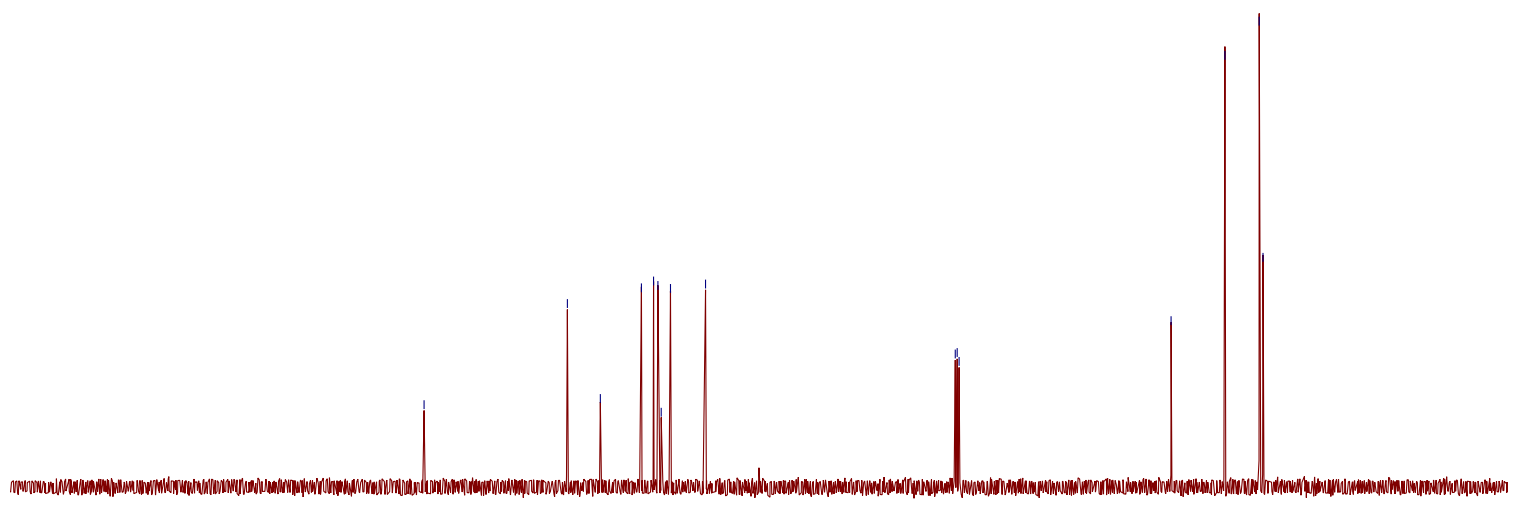

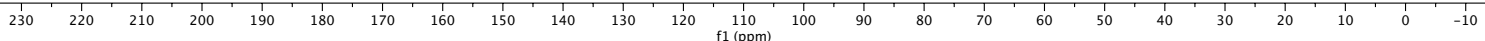


2011746-zh-7-63A3.10.fid

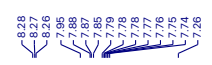<smiles>N#Cc1cc2ccccc2c(C2CCCCC2)n1</smiles>
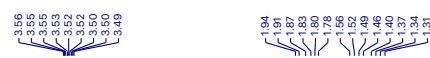

19

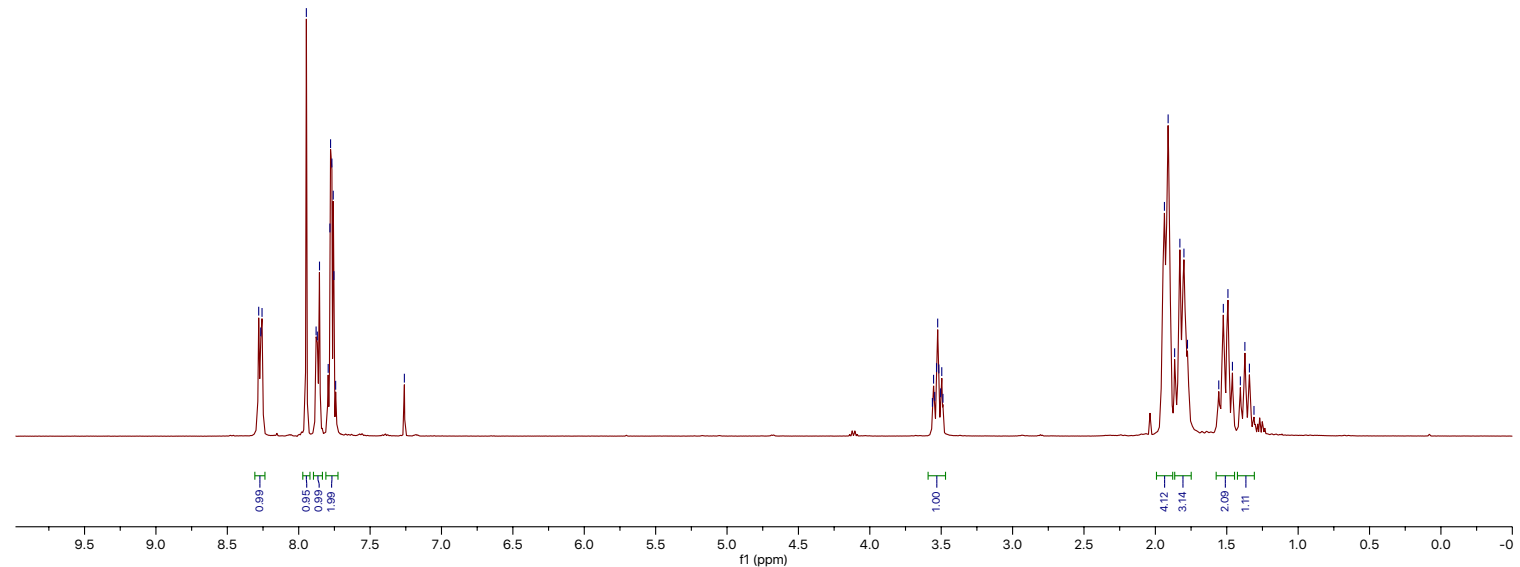

zh-7-60A3

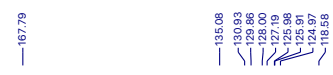

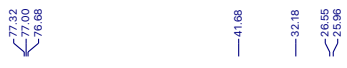

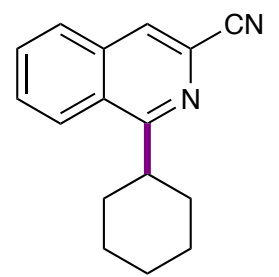

19

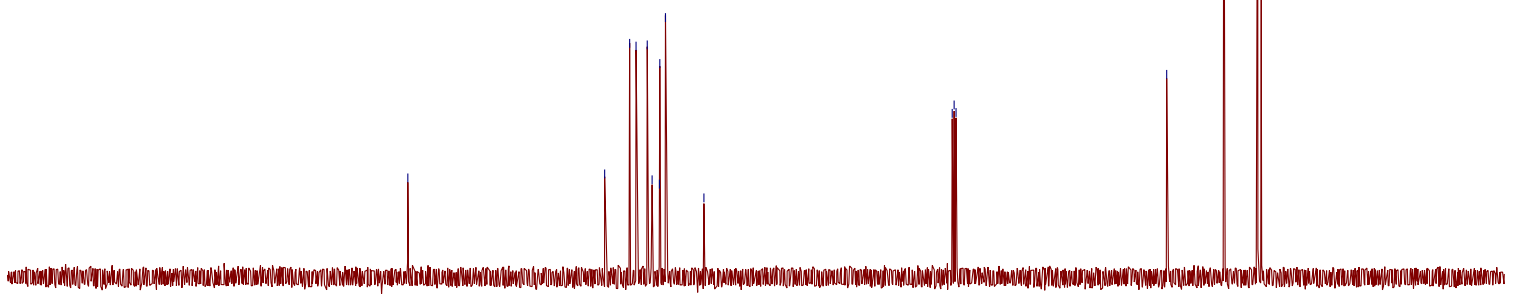

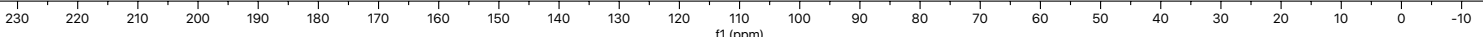



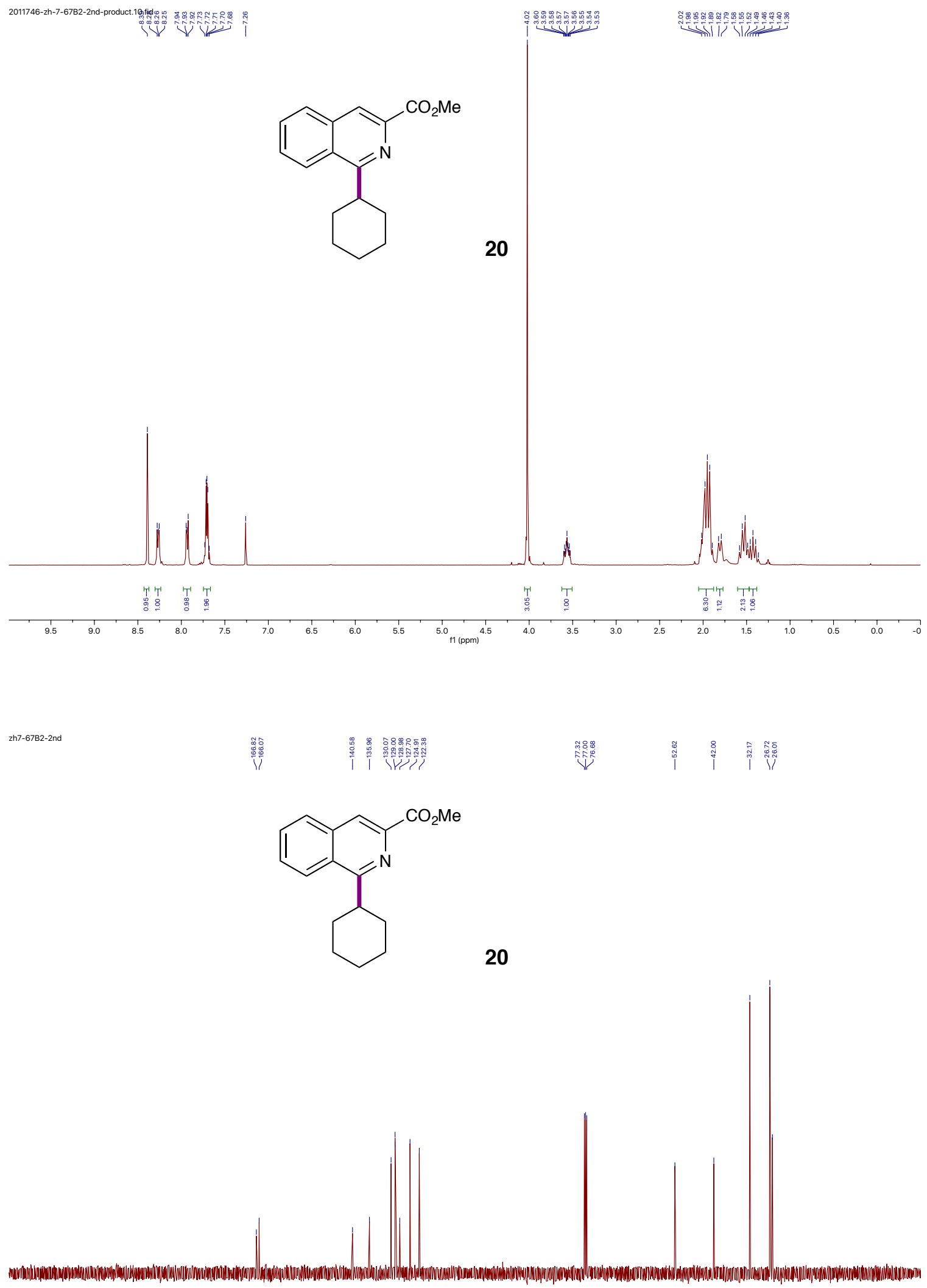

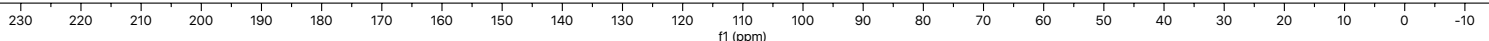




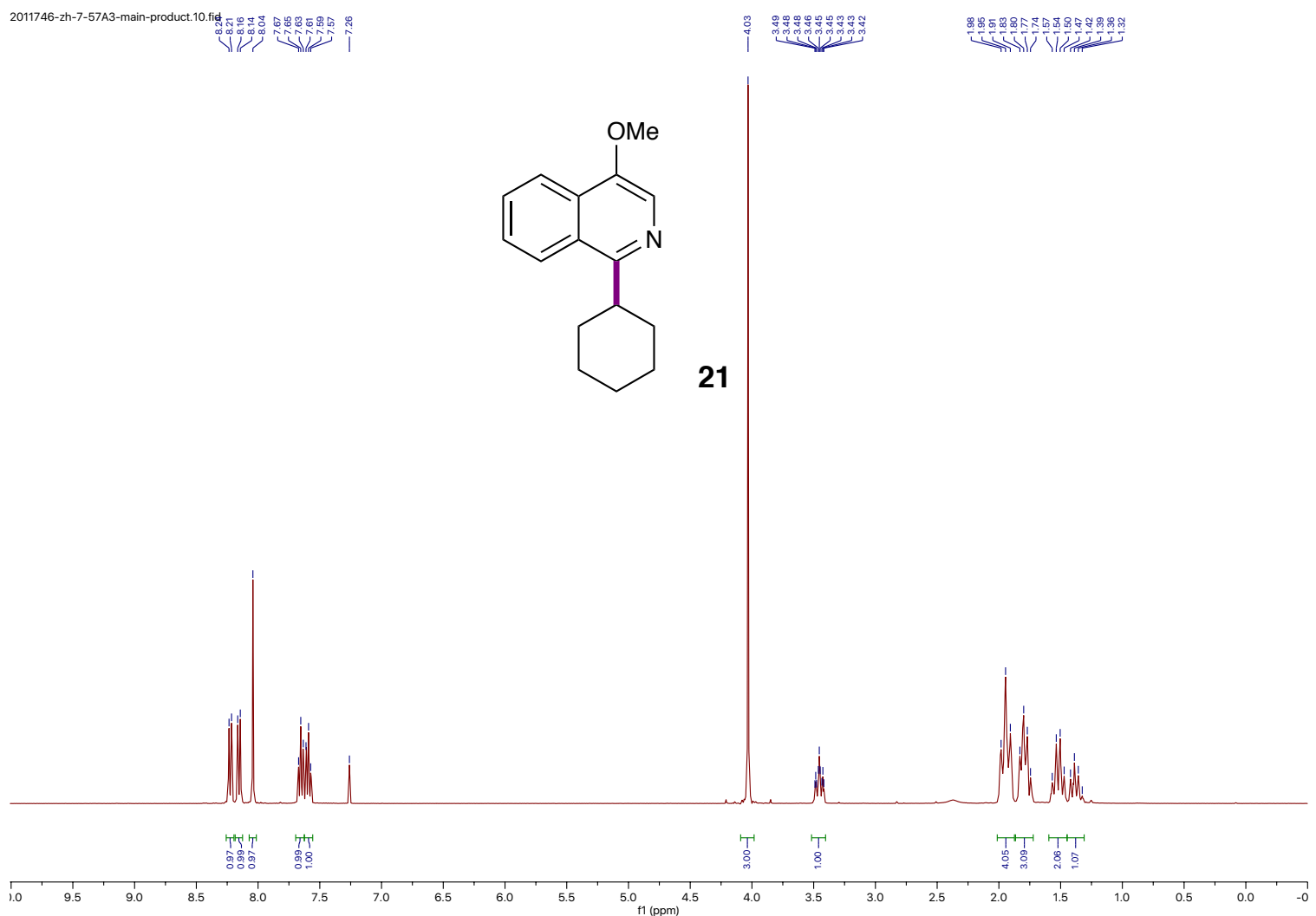

zh-7-57A3

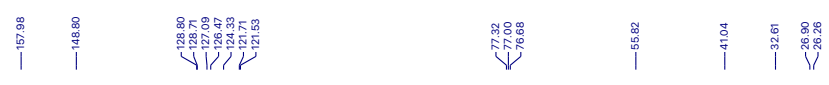<smiles>COc1cnc(C2CCCCC2)c2ccccc12</smiles>

21

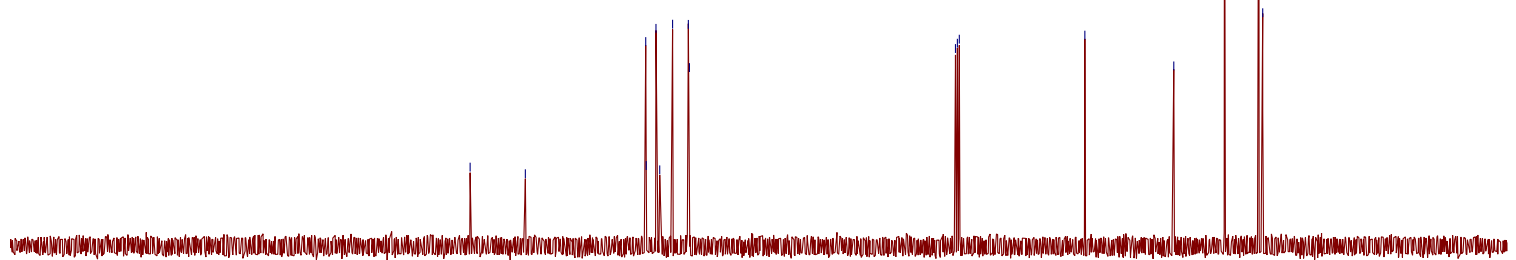

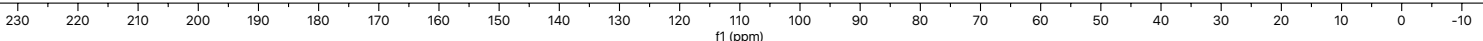


2011746-2h-5-23C-6-7

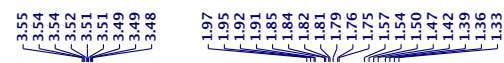<smiles>Brc1cnc(C2CCCCC2)c2ccccc12</smiles>

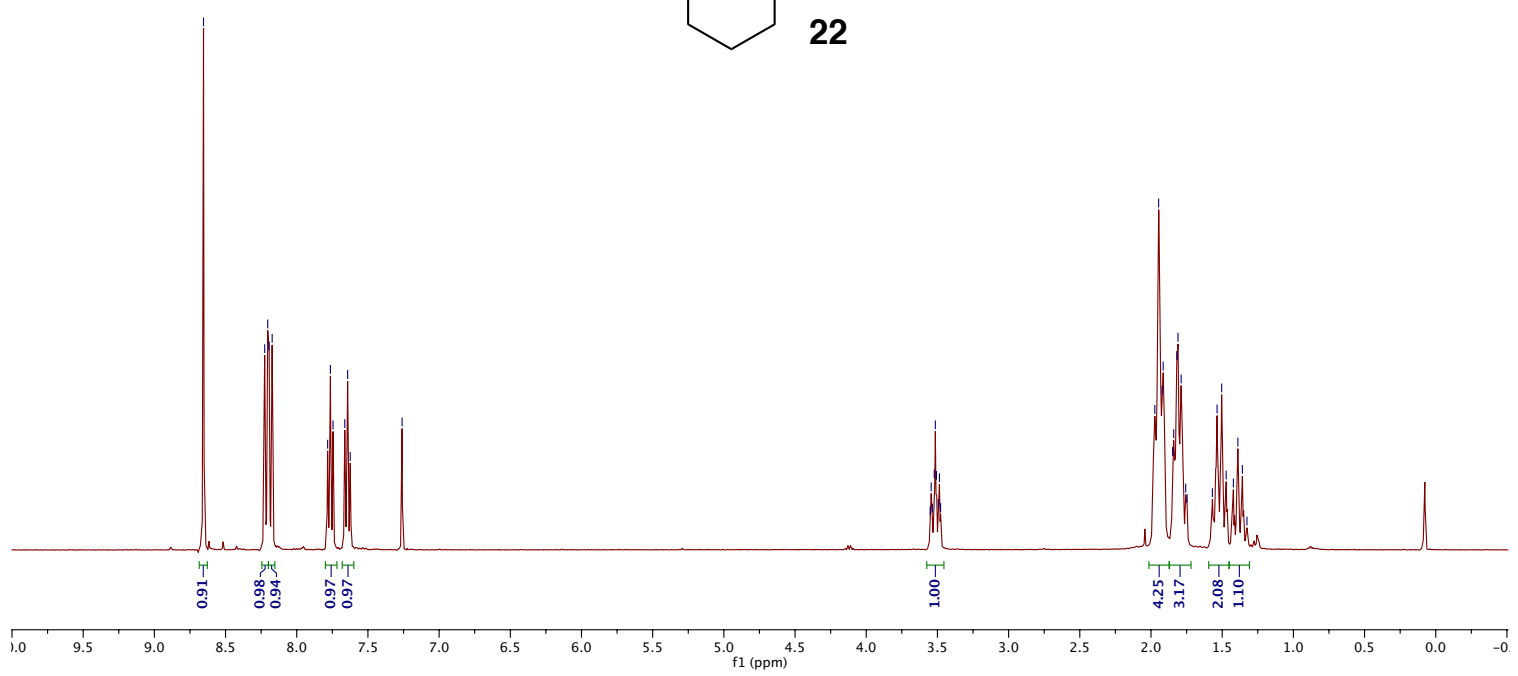

zh-5-23c<smiles>Brc1cnc(C2CCCCC2)c2ccccc12</smiles>

22

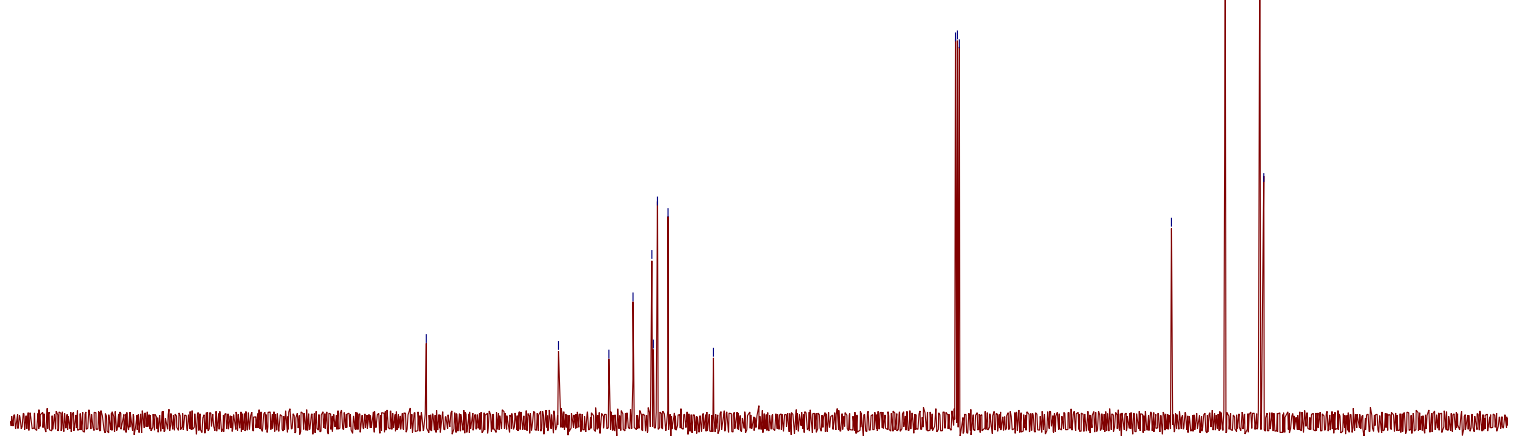

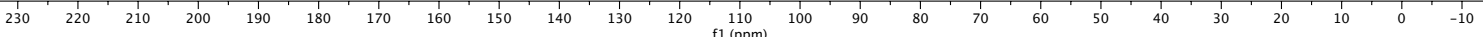


<smiles>C=C(c1ccccc1[N+](=O)[O-])C1CCCCC1</smiles>

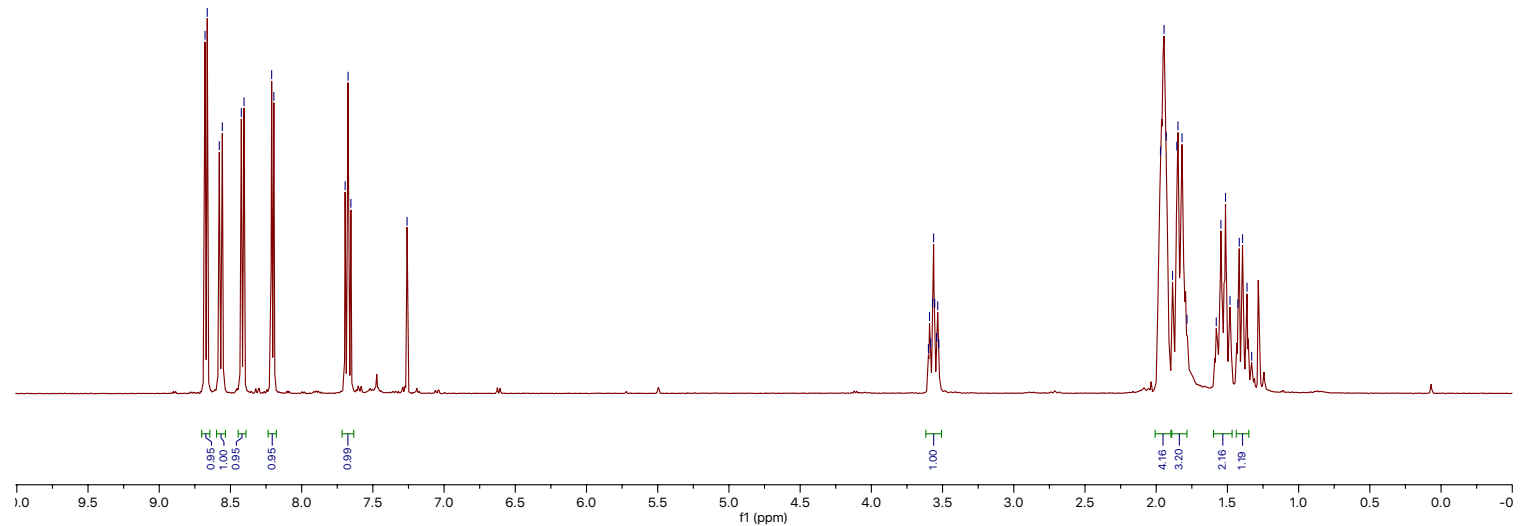

zh7-61a1

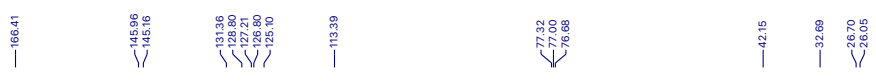<smiles>O=[N+]([O-])c1cccc2c(C3CCCCC3)nccc12</smiles>

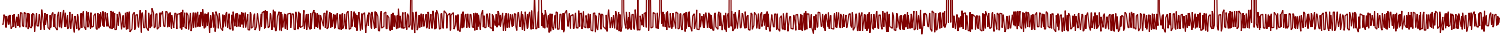

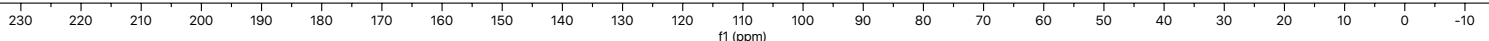


<smiles>c1ccc2c(c1)nc(C1CCCCC1)c1ccccc12</smiles>

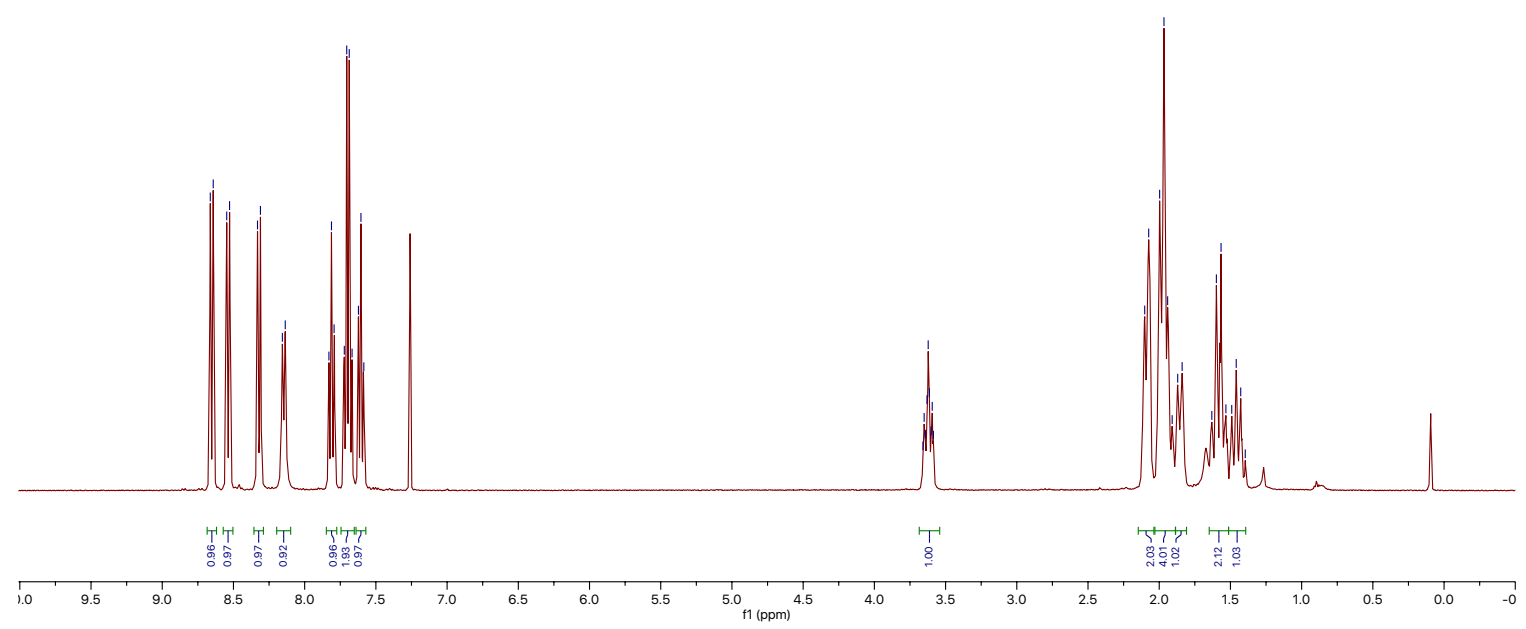

zh-7-33a
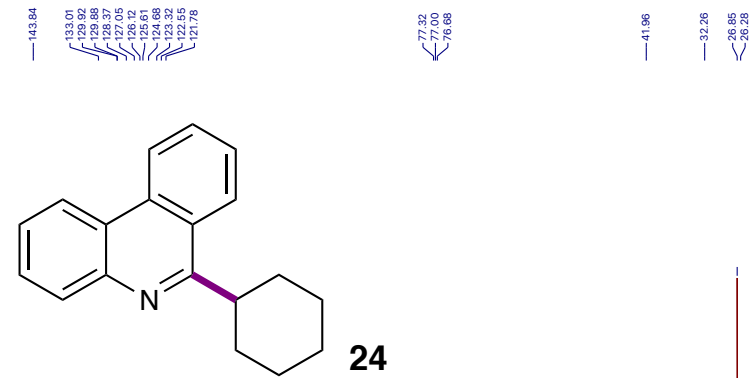

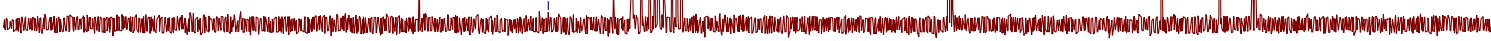

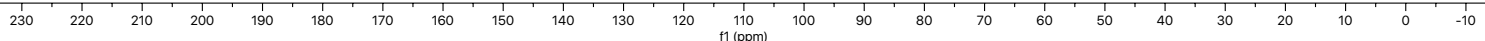




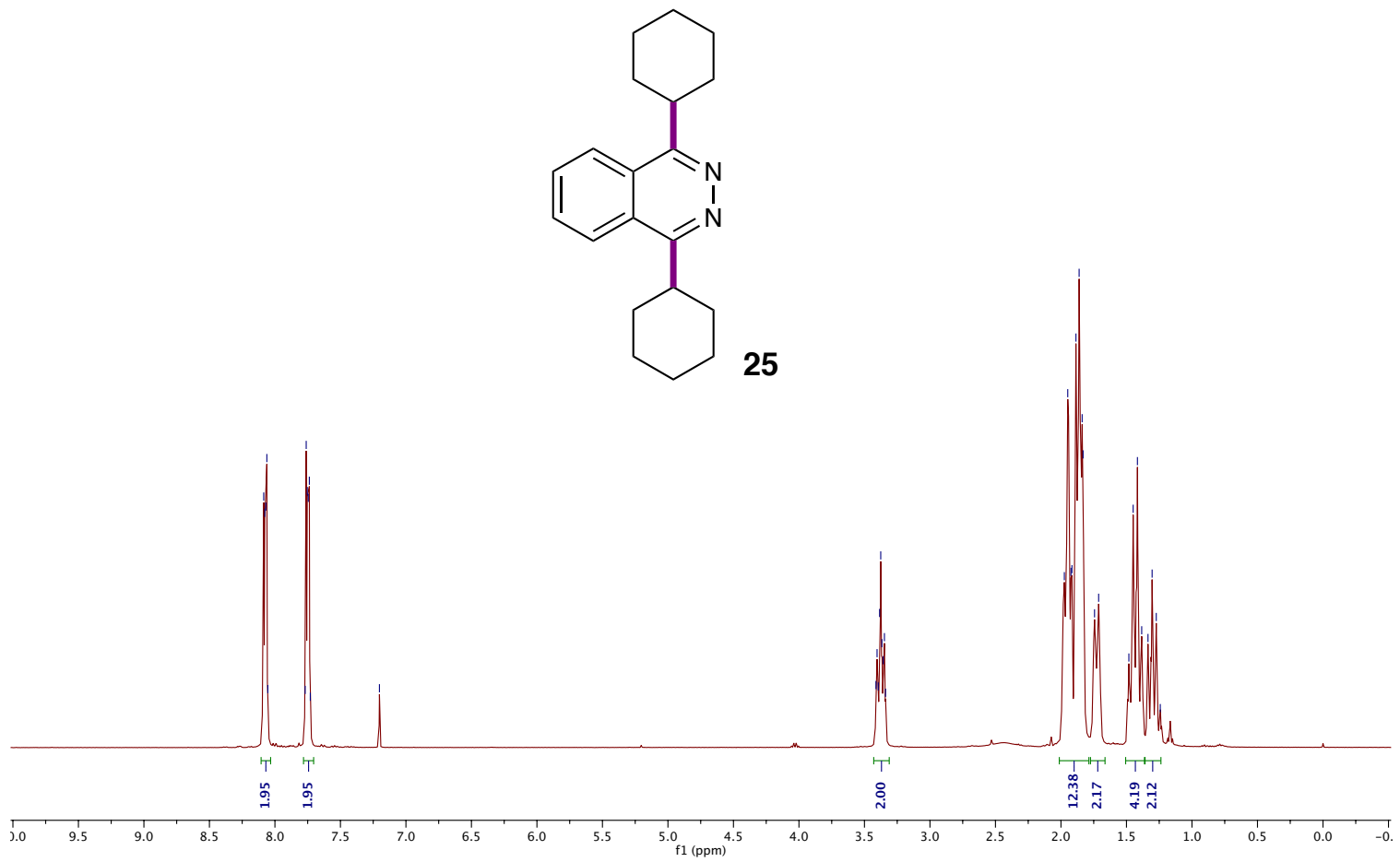

zh-5-90c<smiles>c1ccc2c(C3CCCCC3)nnc(C3CCCCC3)c2c1</smiles>

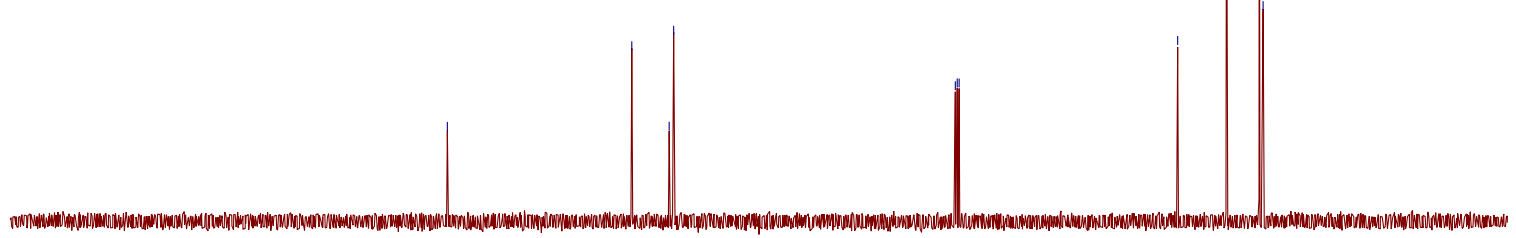

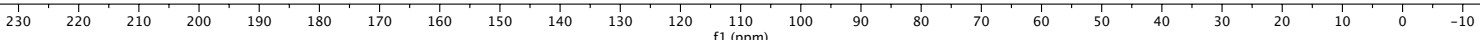


2011746-Zh-7-79A2-product.20.fid
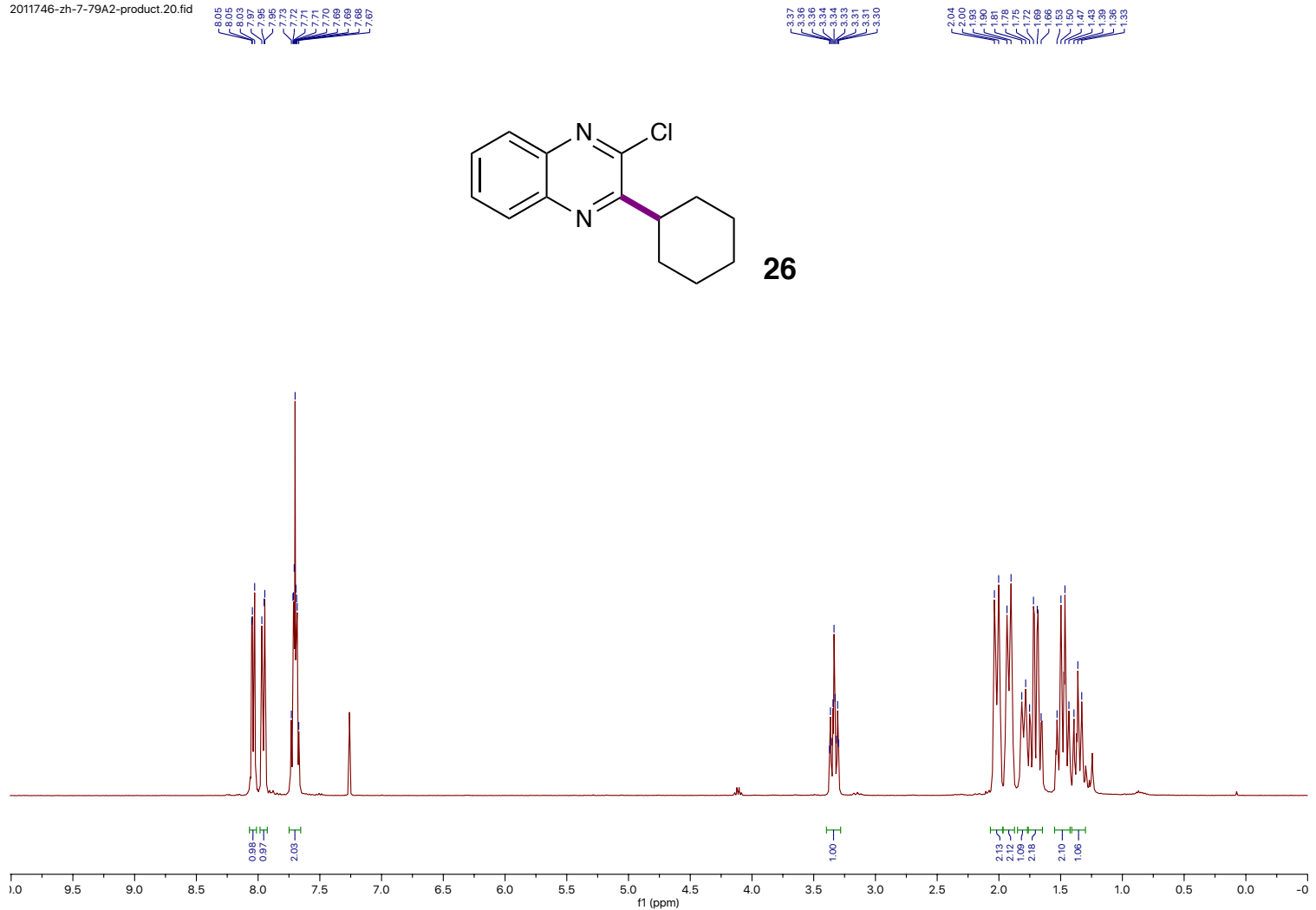

zh7-79A2
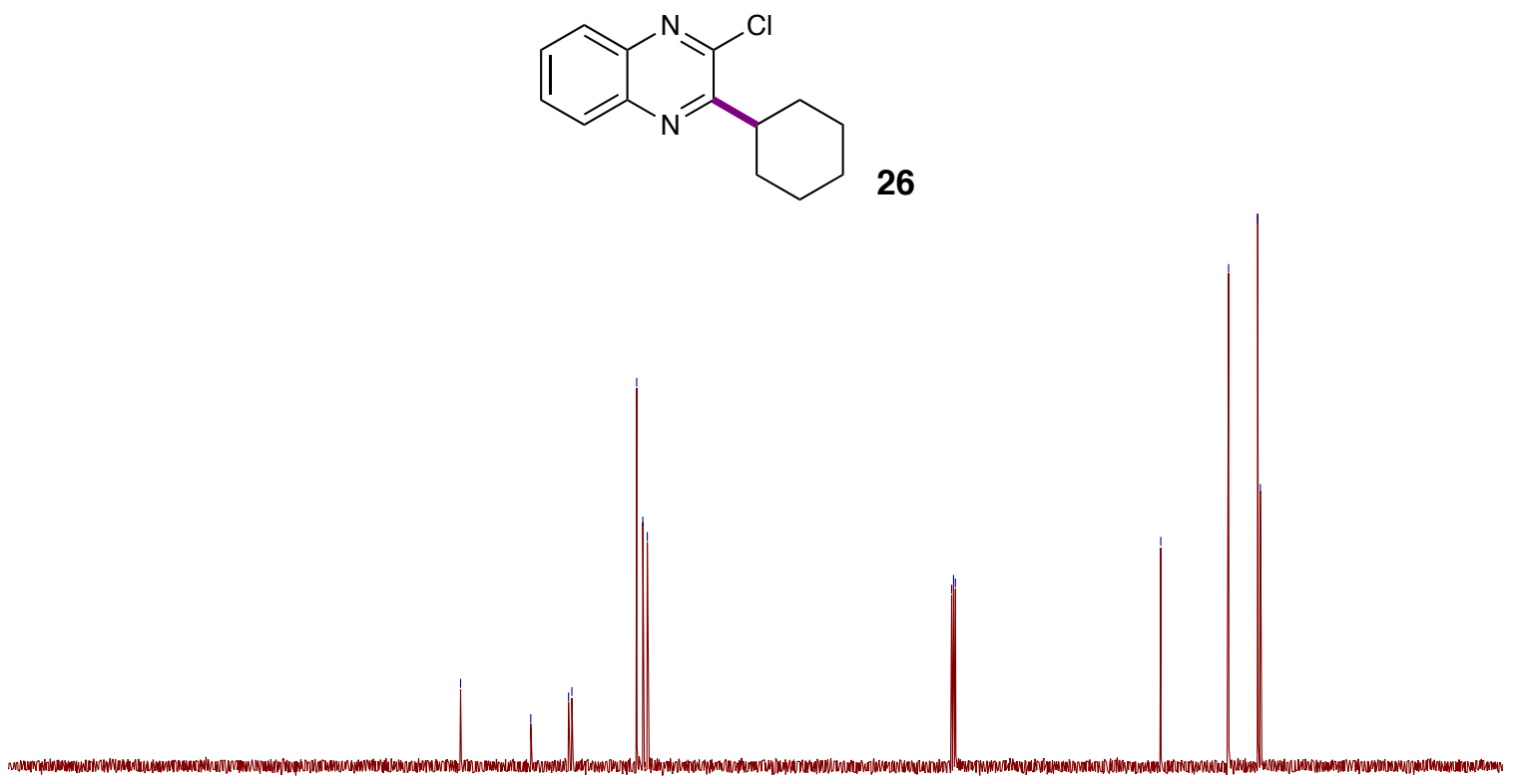

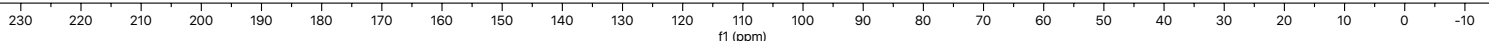


2011746-2h-7-71A2-2nd-product.10.fid
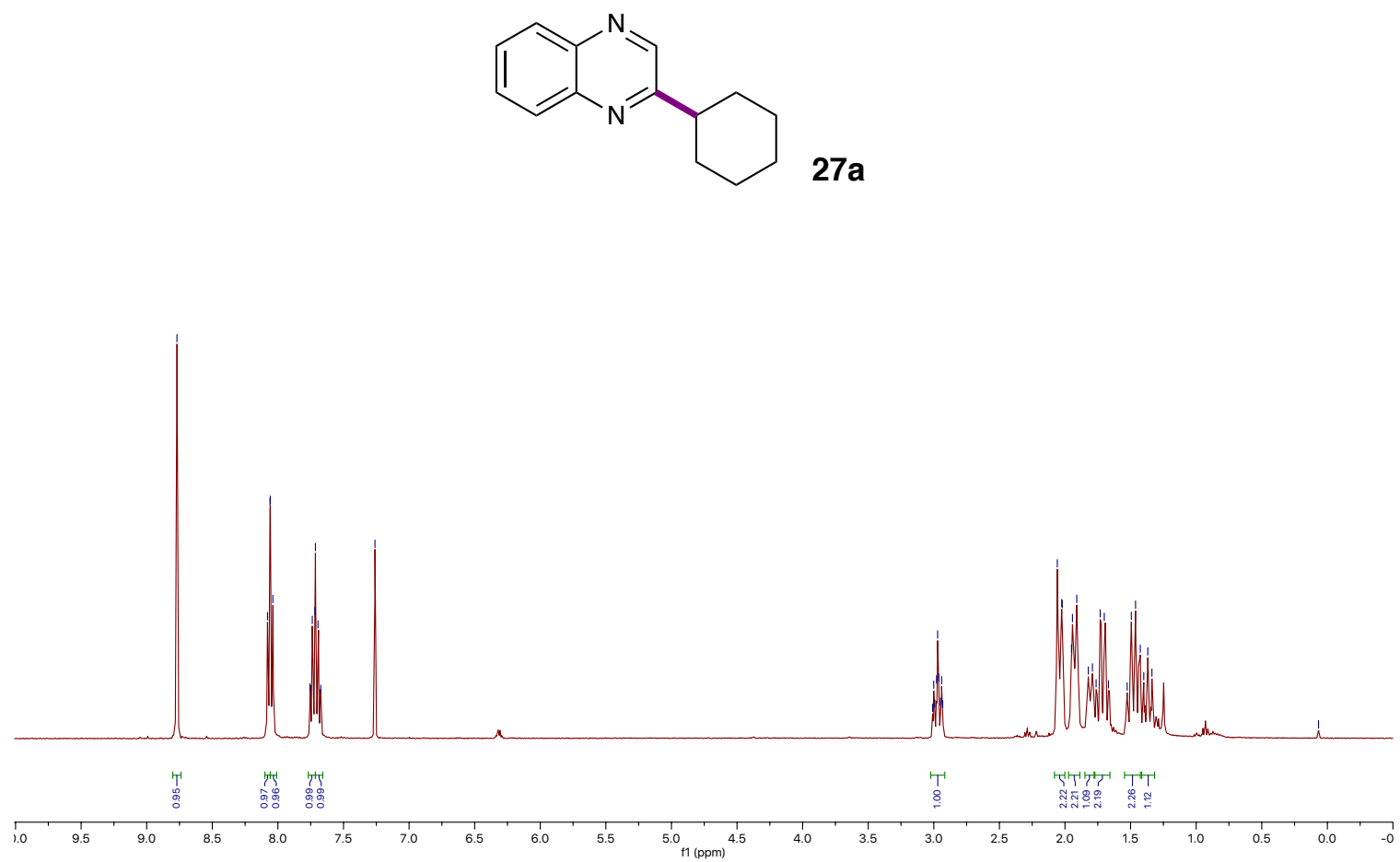

zh7-71a2-2
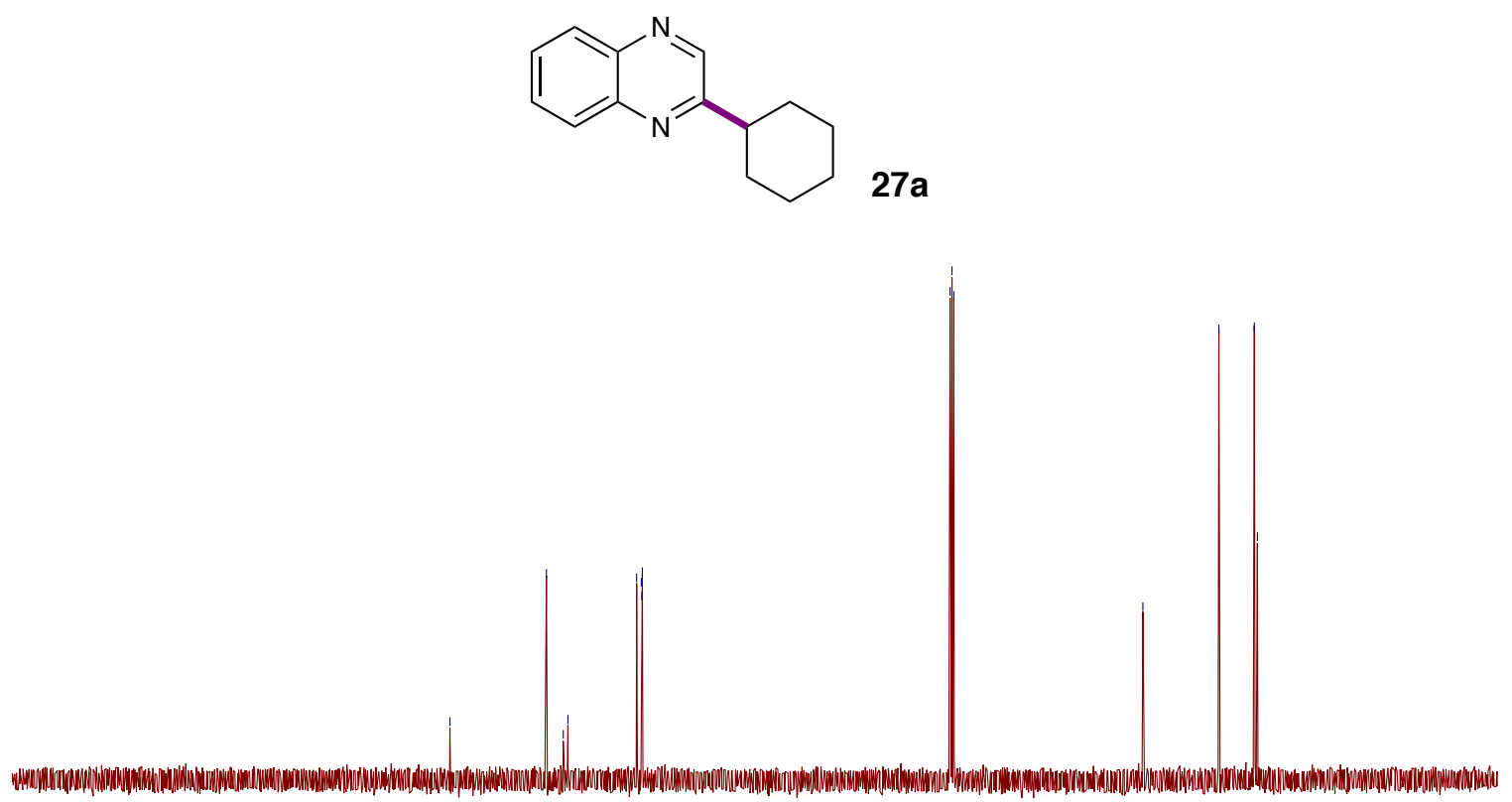

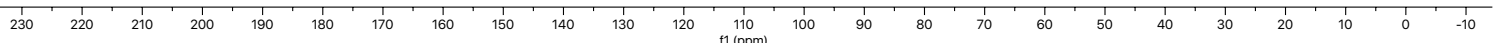


<smiles>c1ccc2nc(C3CCCCC3)c(C3CCCCC3)nc2c1</smiles>

$27 b$

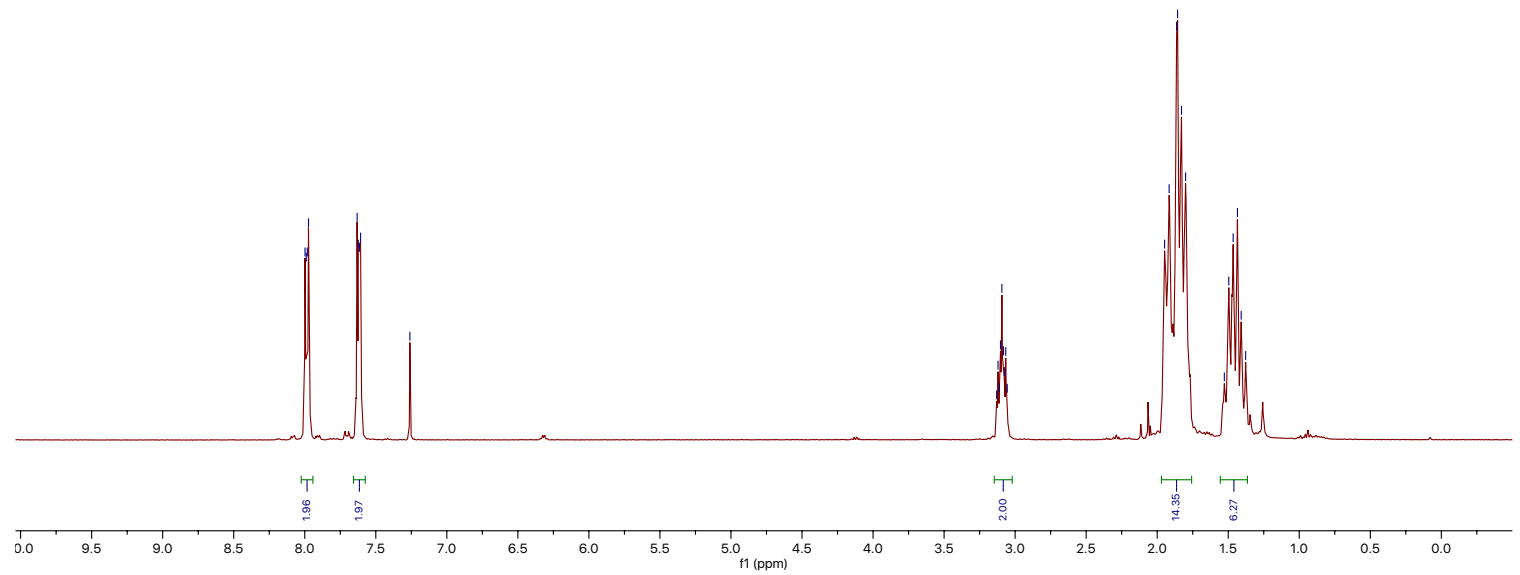

zh-7-71A2-1st

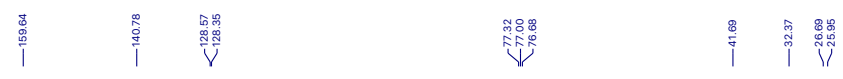<smiles>c1ccc2nc(C3CCCCC3)c(C3CCCCC3)nc2c1</smiles>

$27 b$

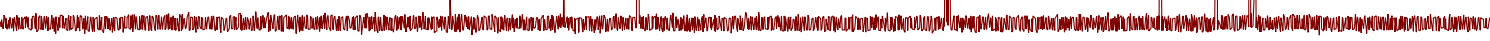

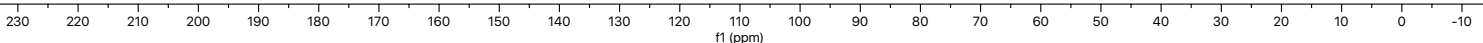



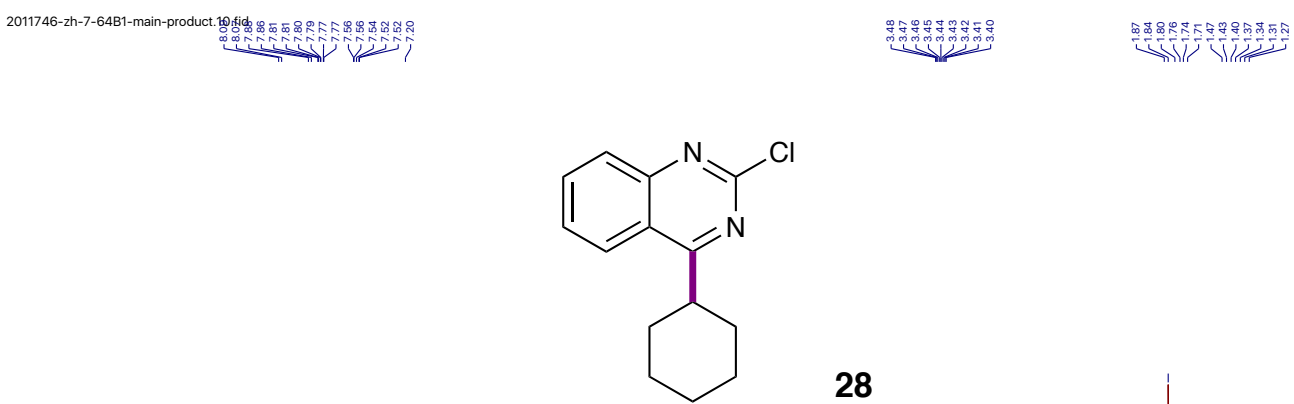

28

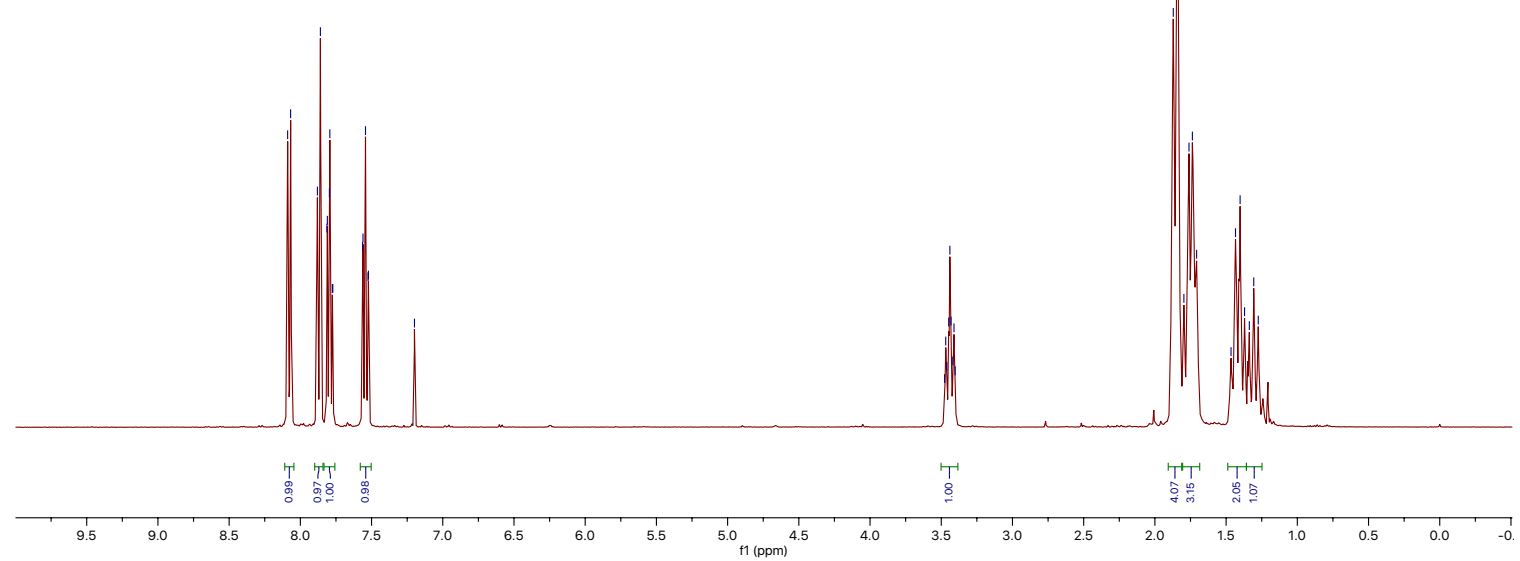

zh7-64B1

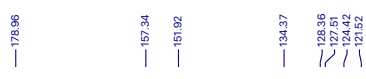

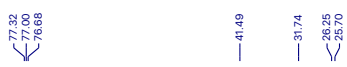<smiles>Clc1nc(C2CCCCC2)c2ccccc2n1</smiles>

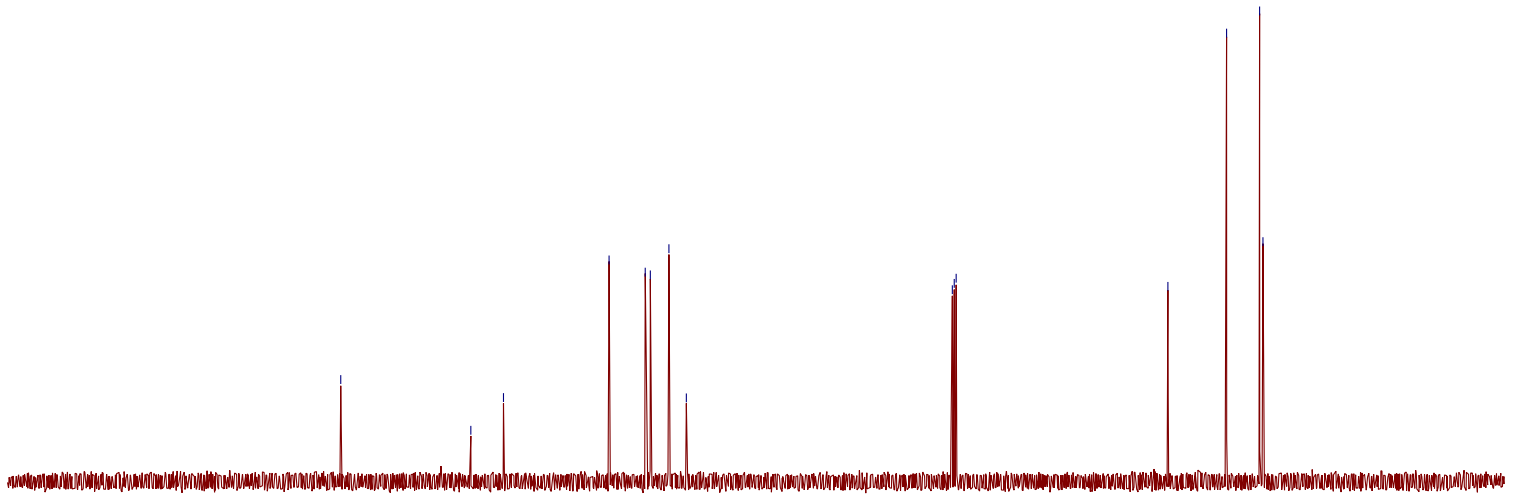

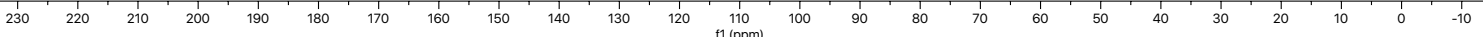


<smiles>c1ccc2c(C3CCCCC3)ncnc2c1</smiles>

$29 a$

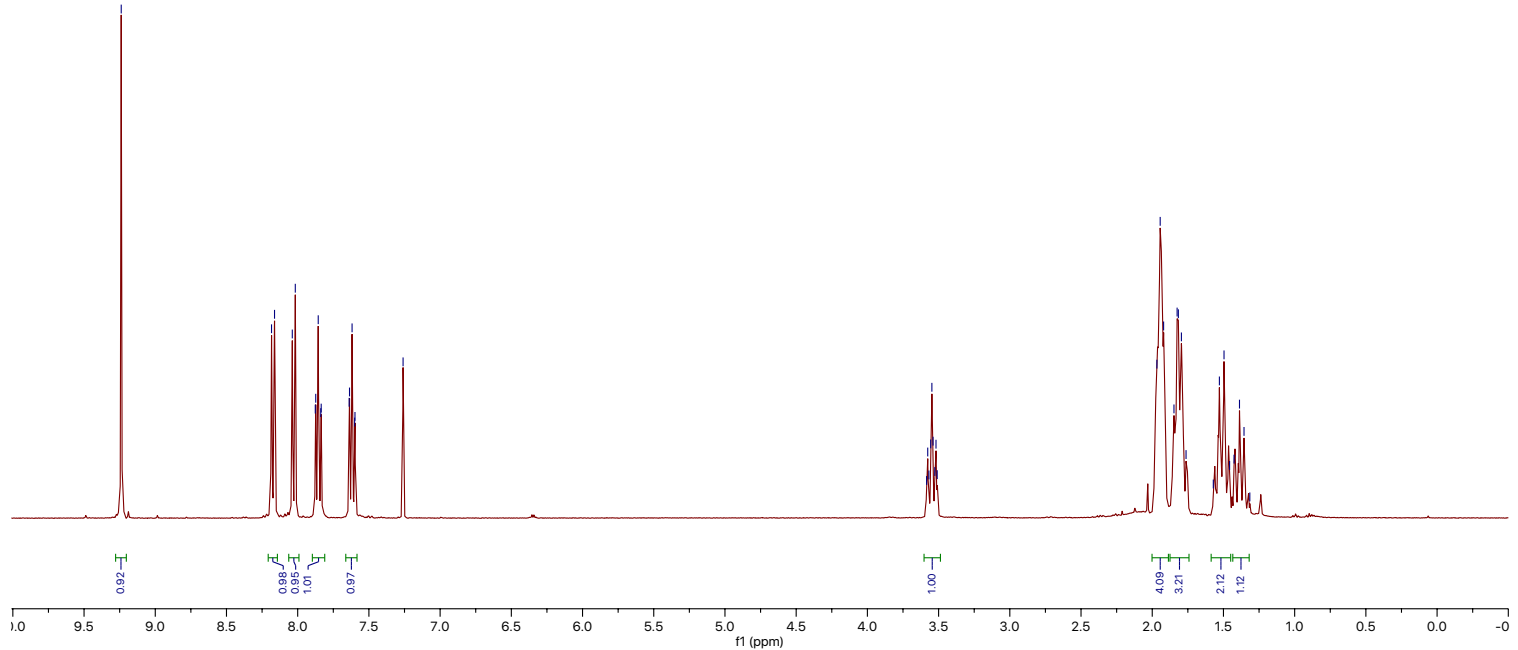

zh7-73a2-2

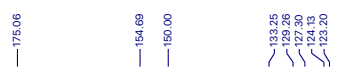

每<smiles>c1ccc2c(C3CCCCC3)ncnc2c1</smiles>

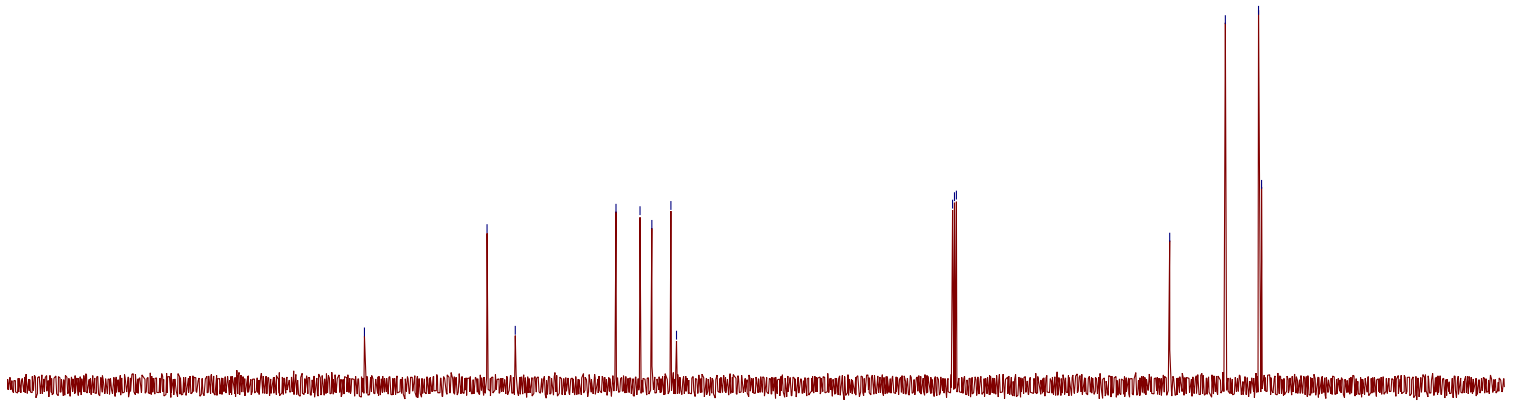
$180 \quad 170 \quad 160 \quad 150$

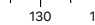

$\operatorname{110}_{11(\mathrm{pm})}^{10} 100$ 


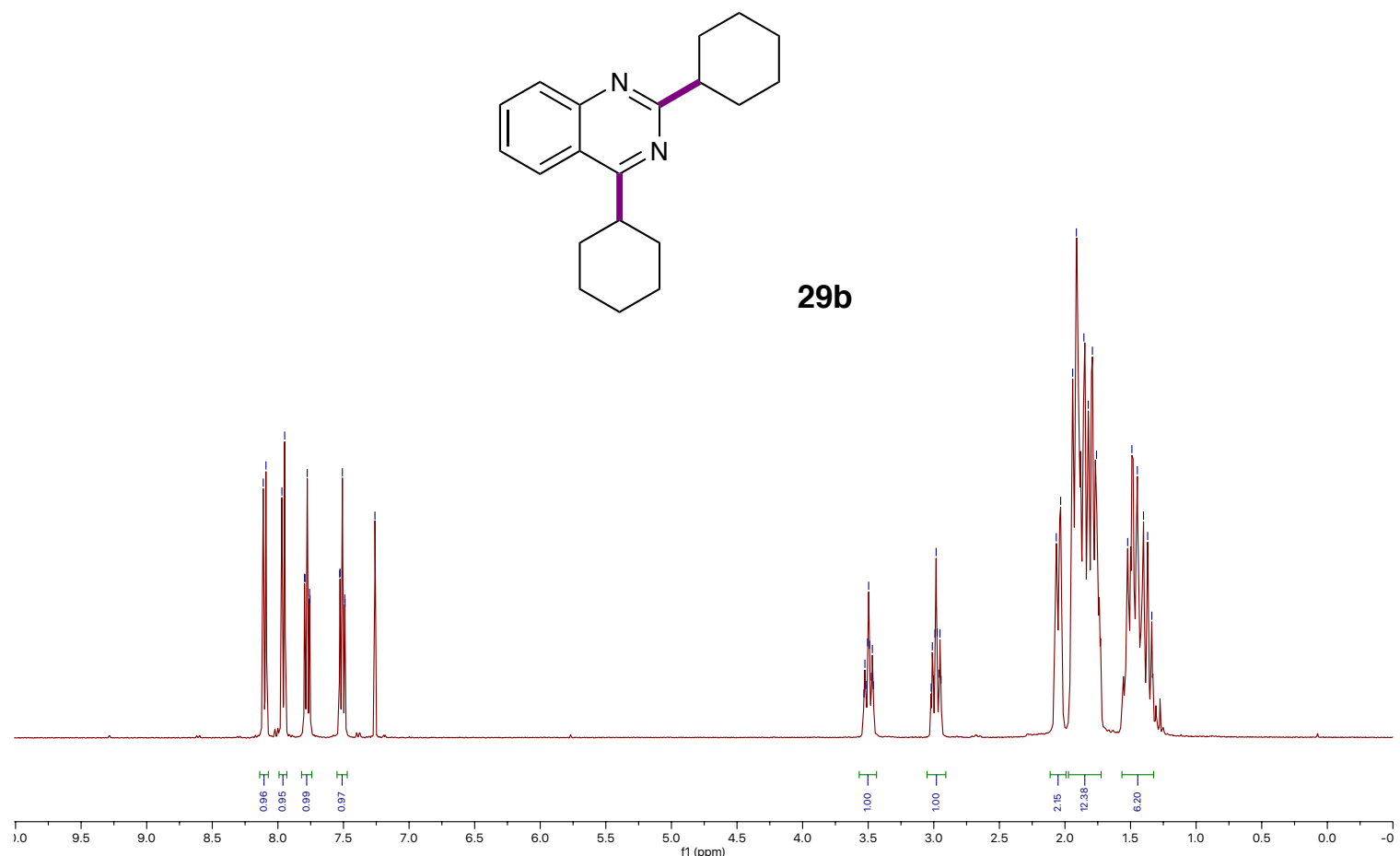

zh5-91c2
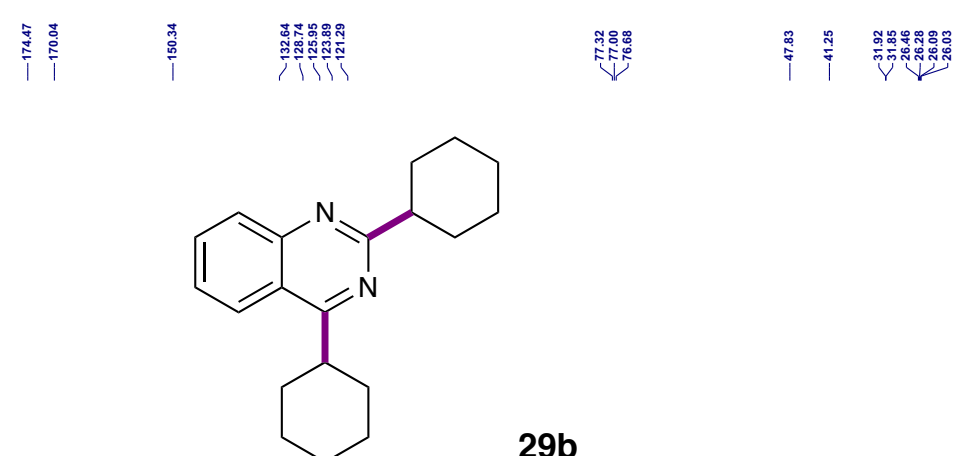

$29 b$

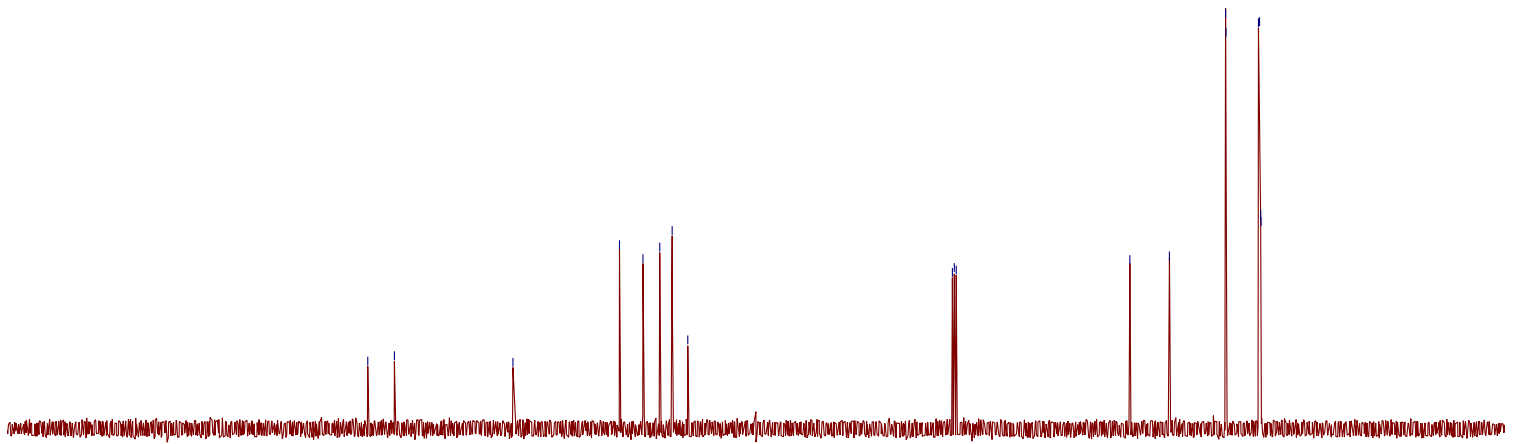

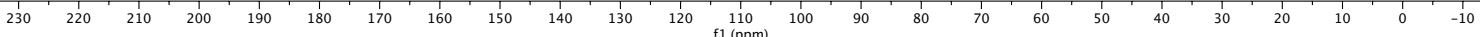




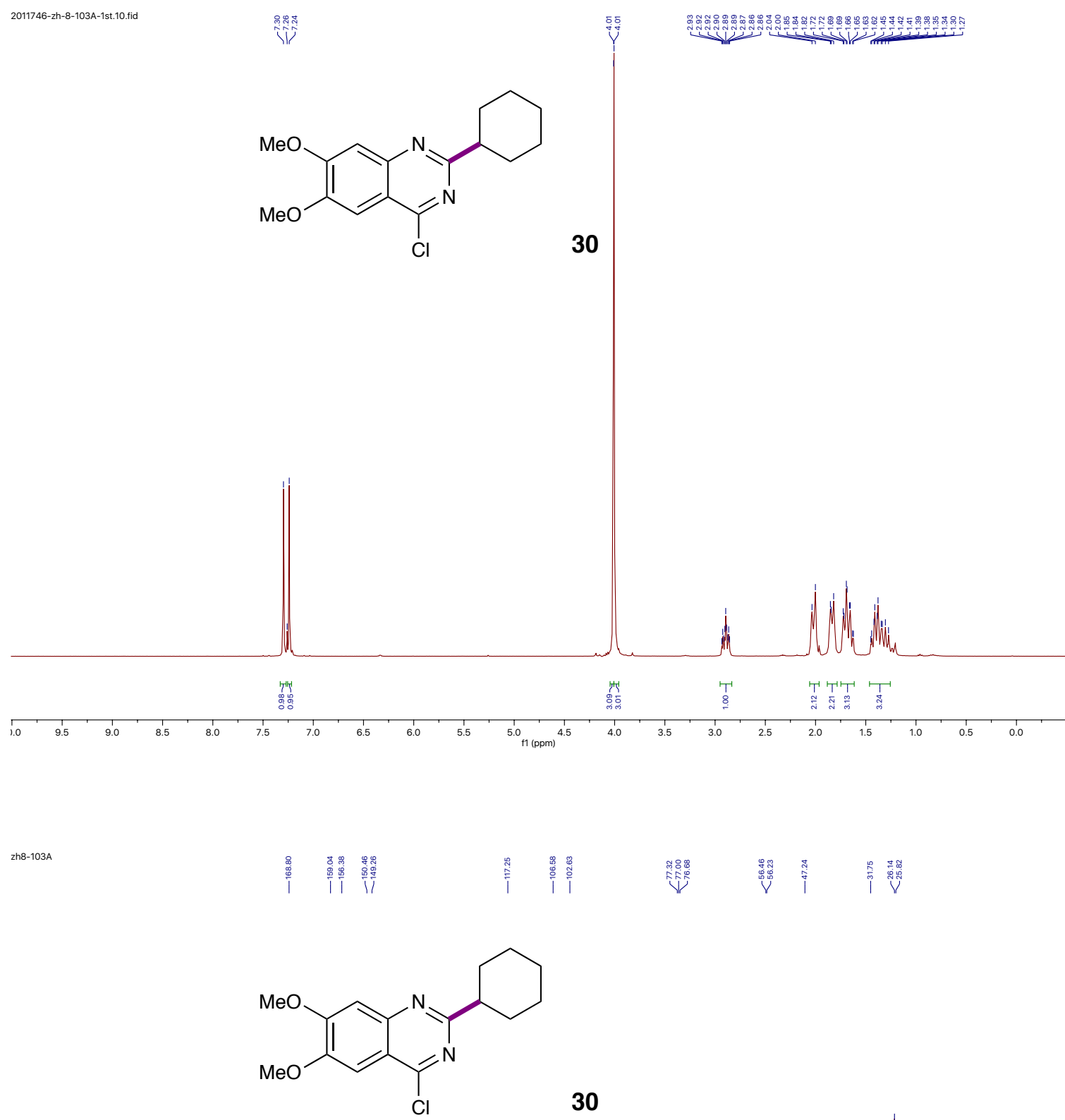

30

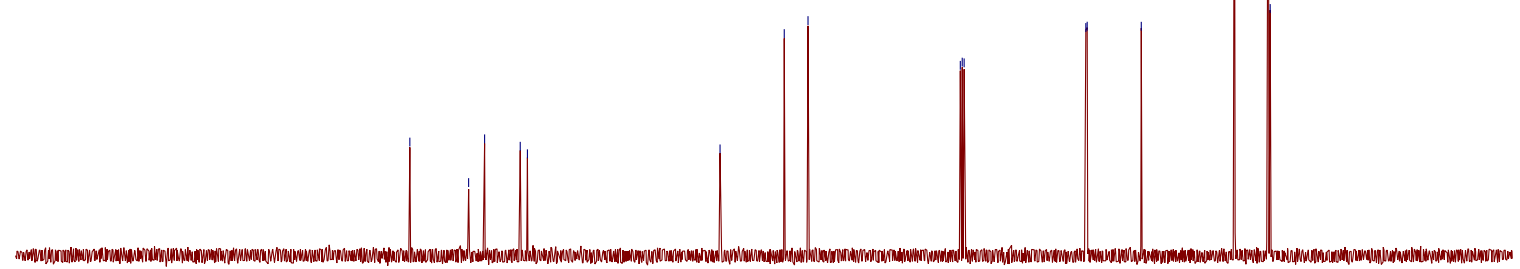

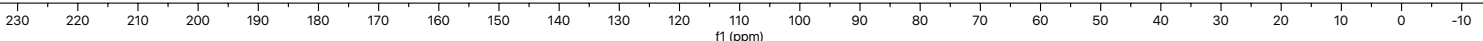



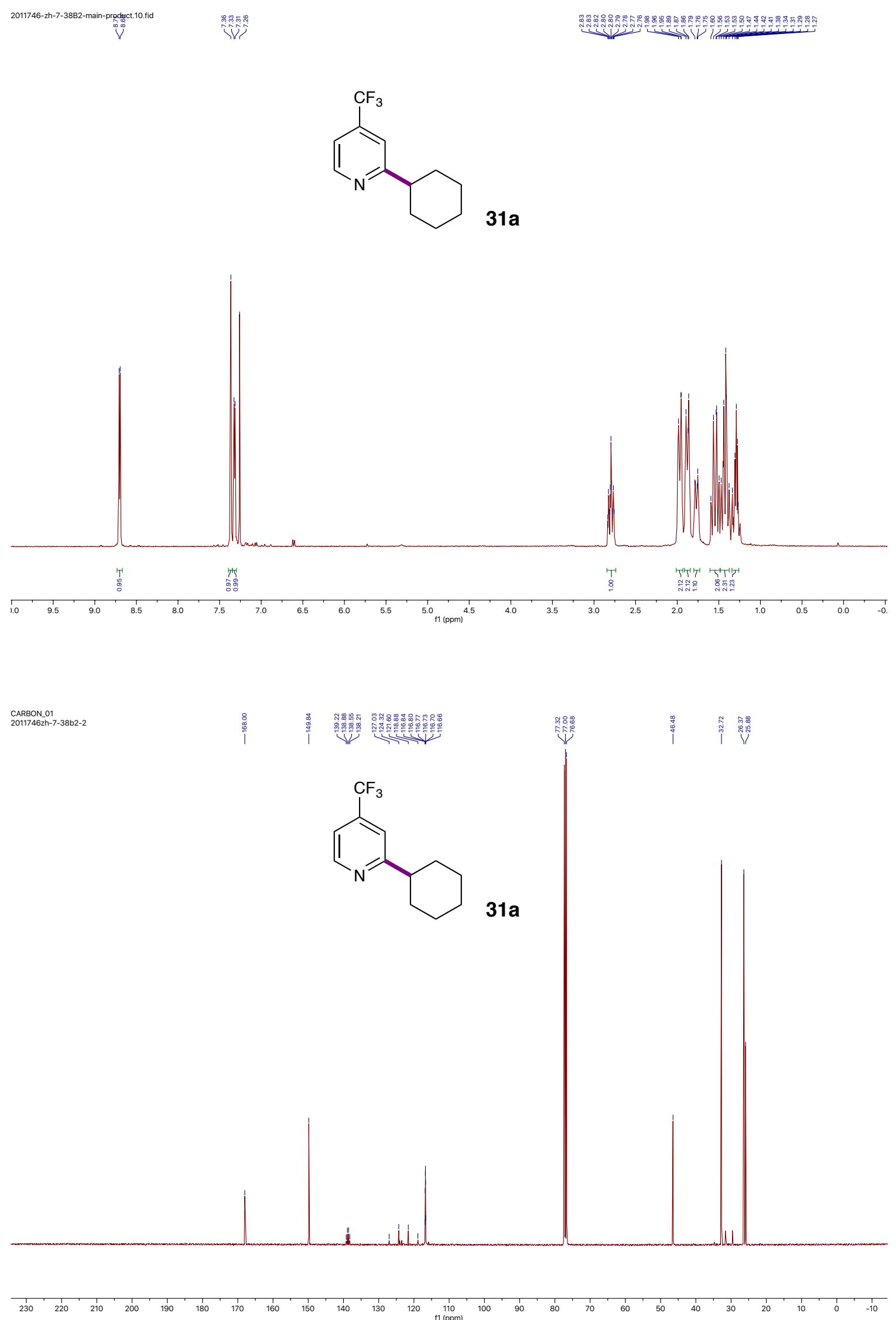

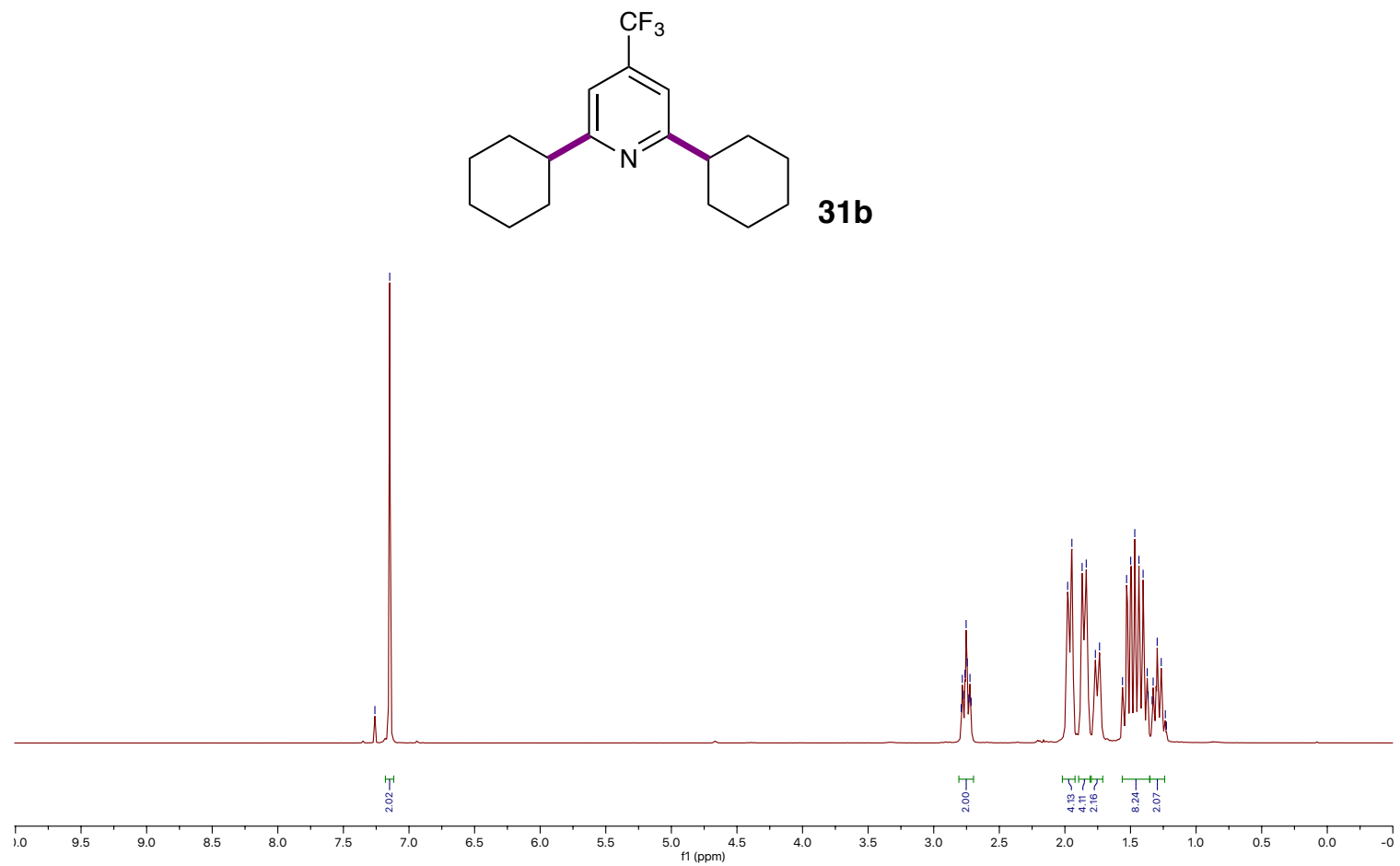

zh-7-38a1<smiles>FC(F)(F)c1cc(C2CCCCC2)nc(C2CCCCC2)c1</smiles>

31b

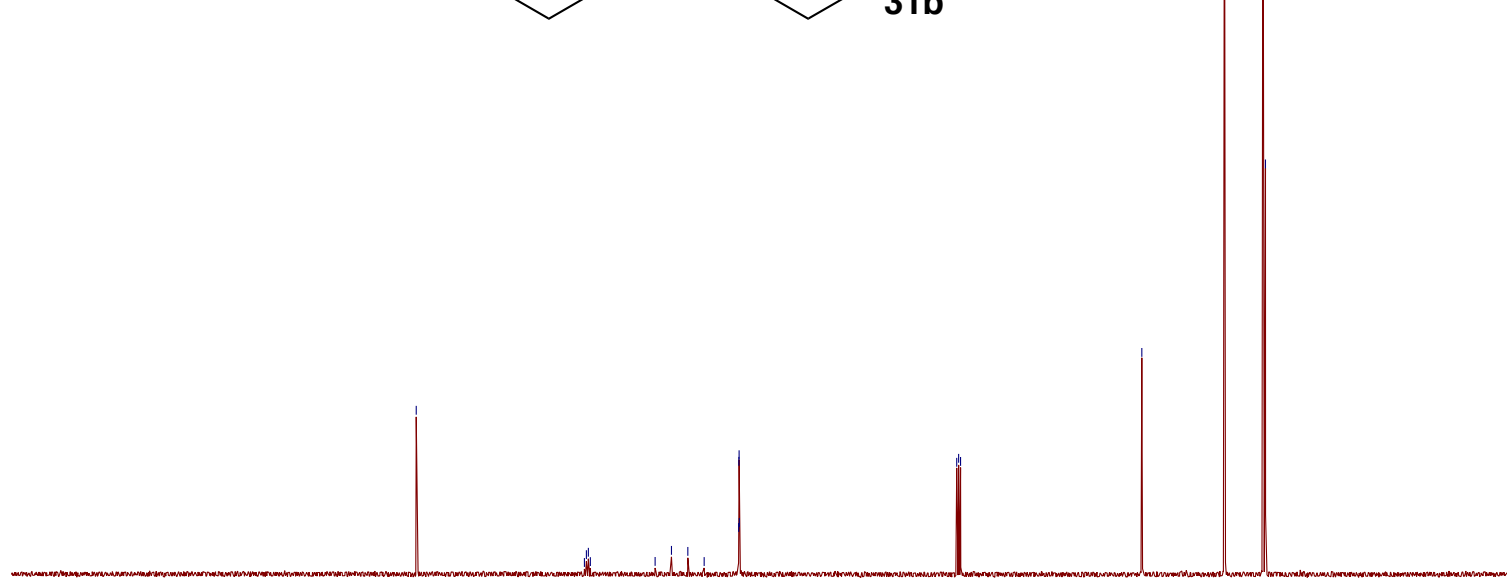

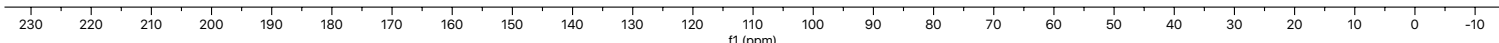



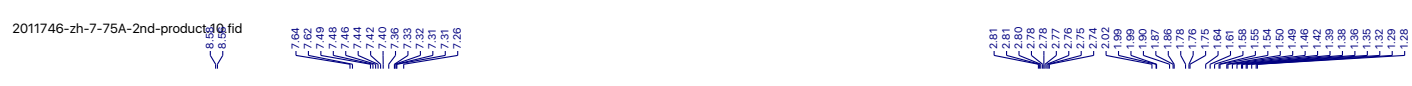<smiles>c1ccc(-c2ccnc(C3CCCCC3)c2)cc1</smiles>

$32 a$

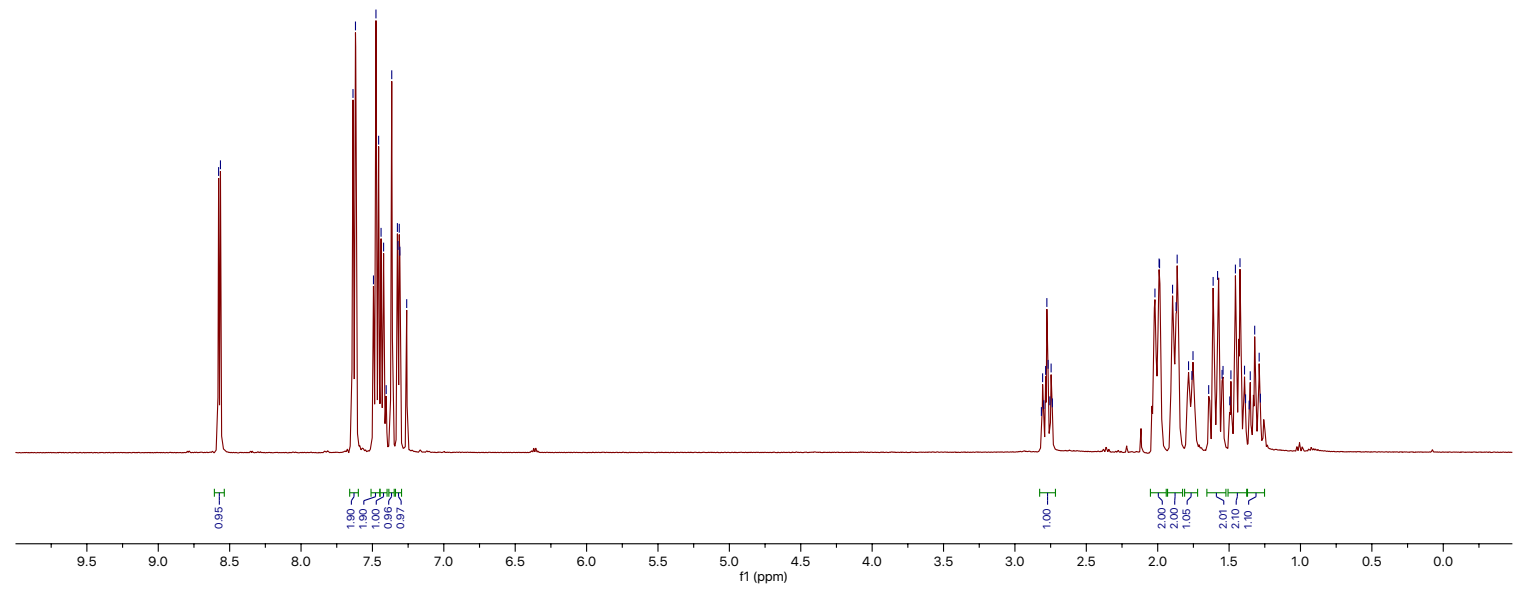

zh7-75a2
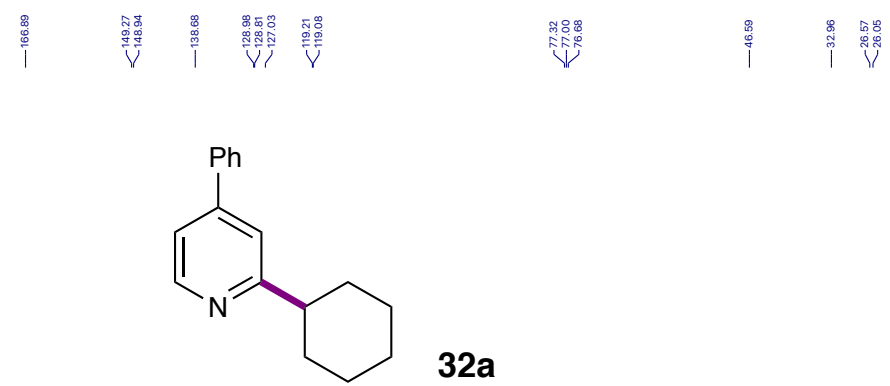

$32 a$

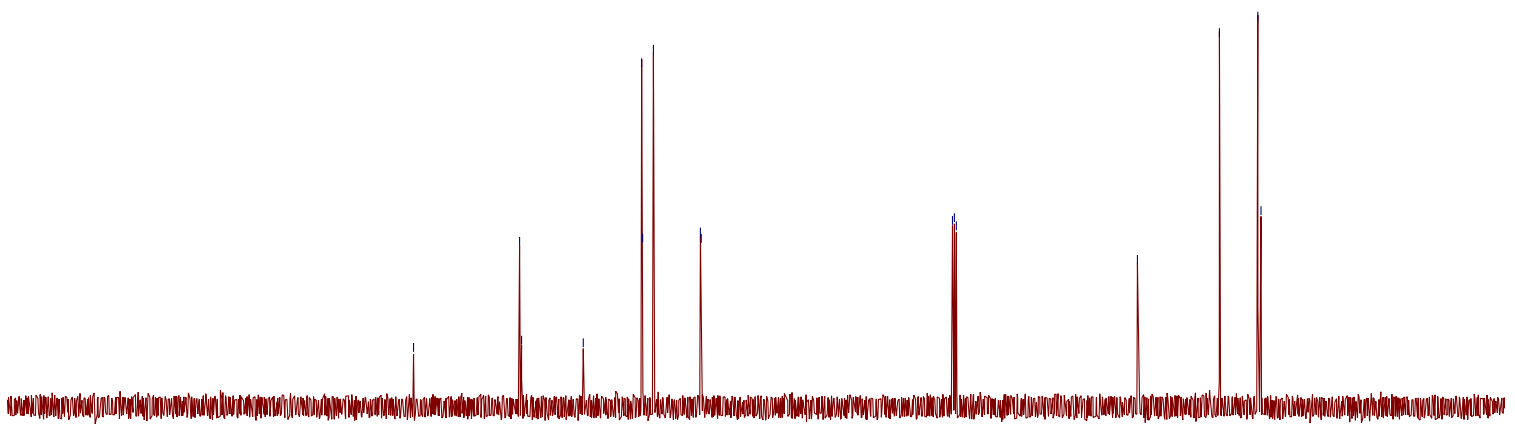

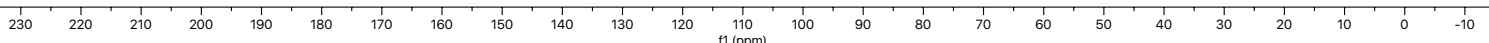



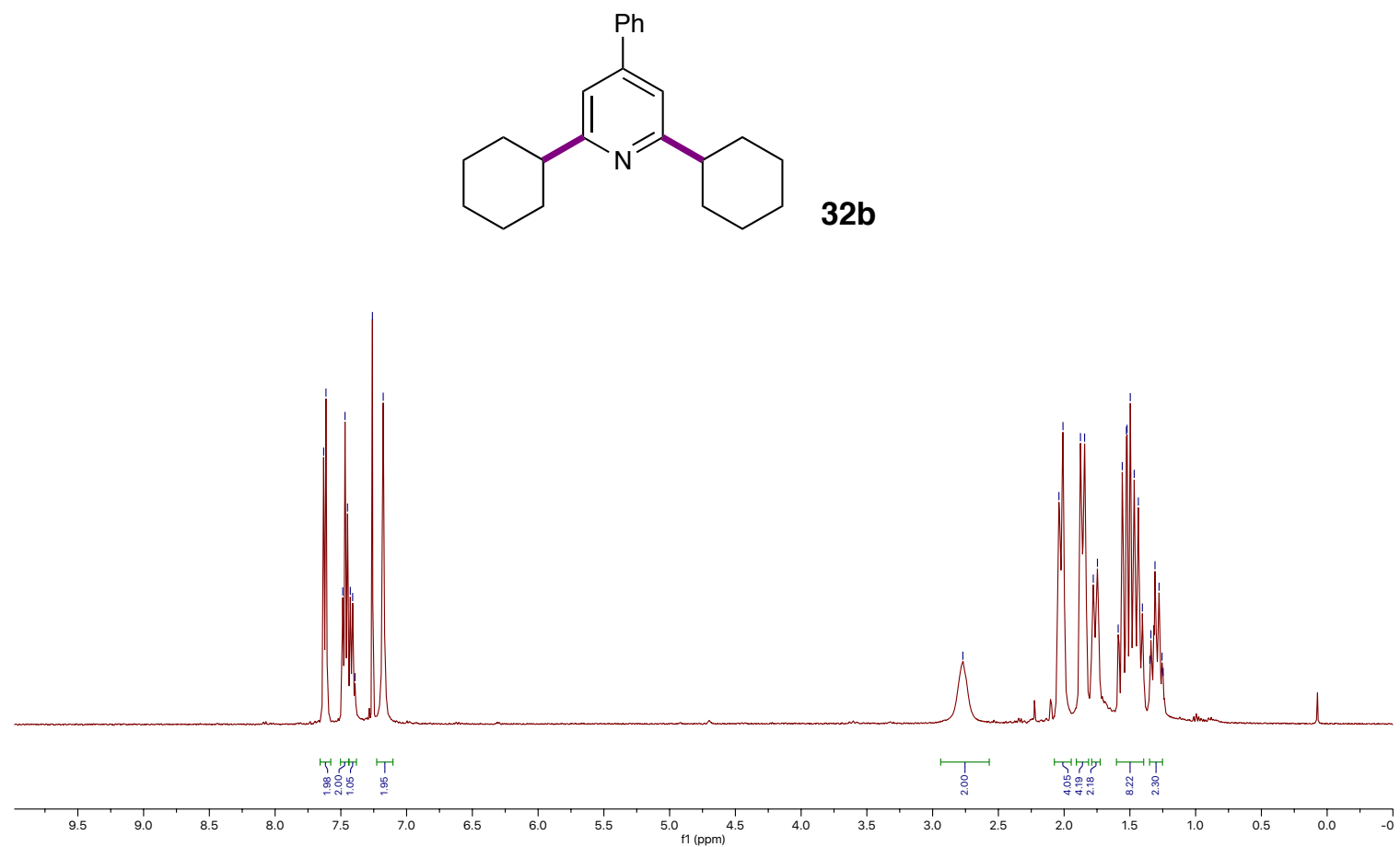

zh7-75a1
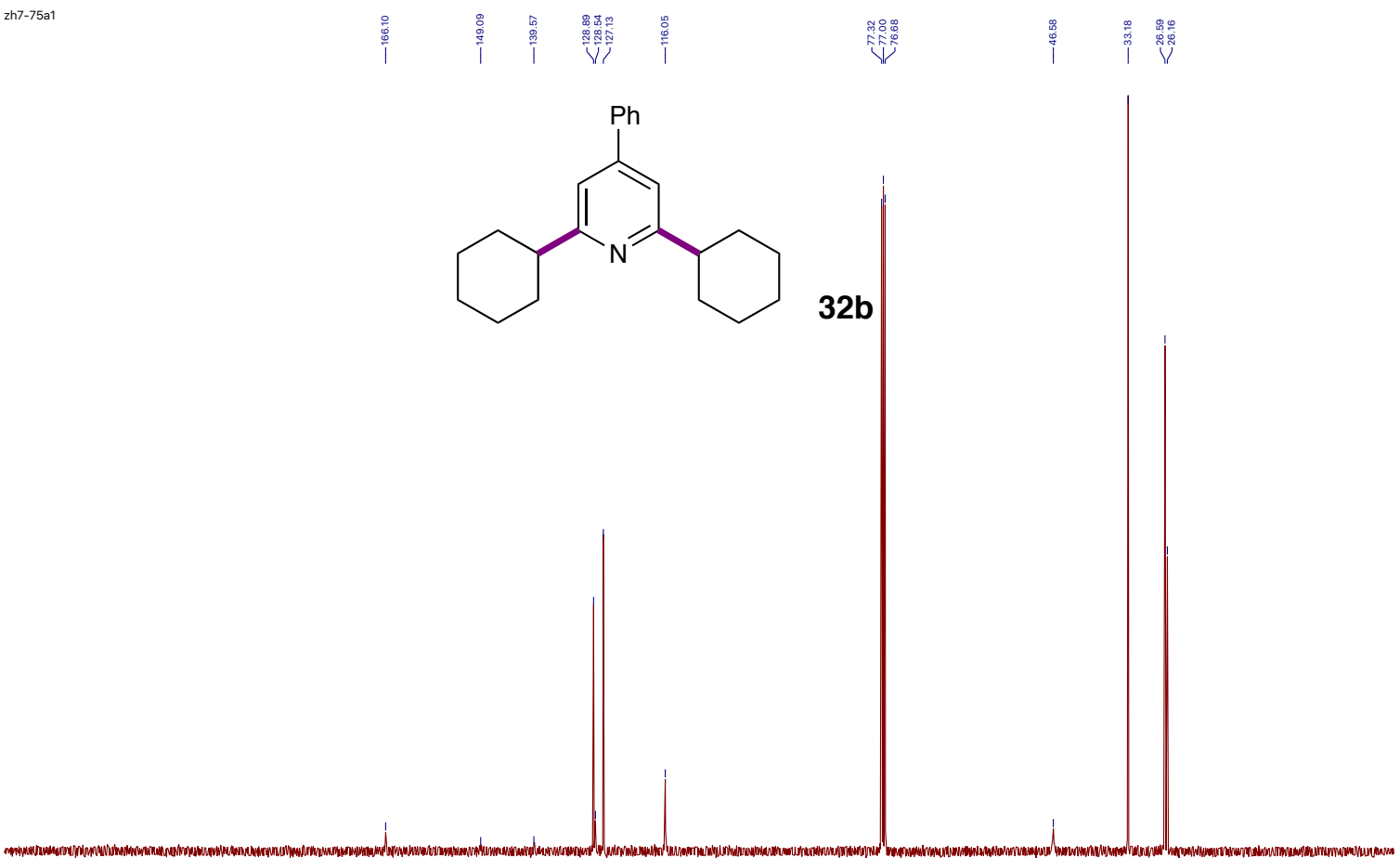

$32 b$

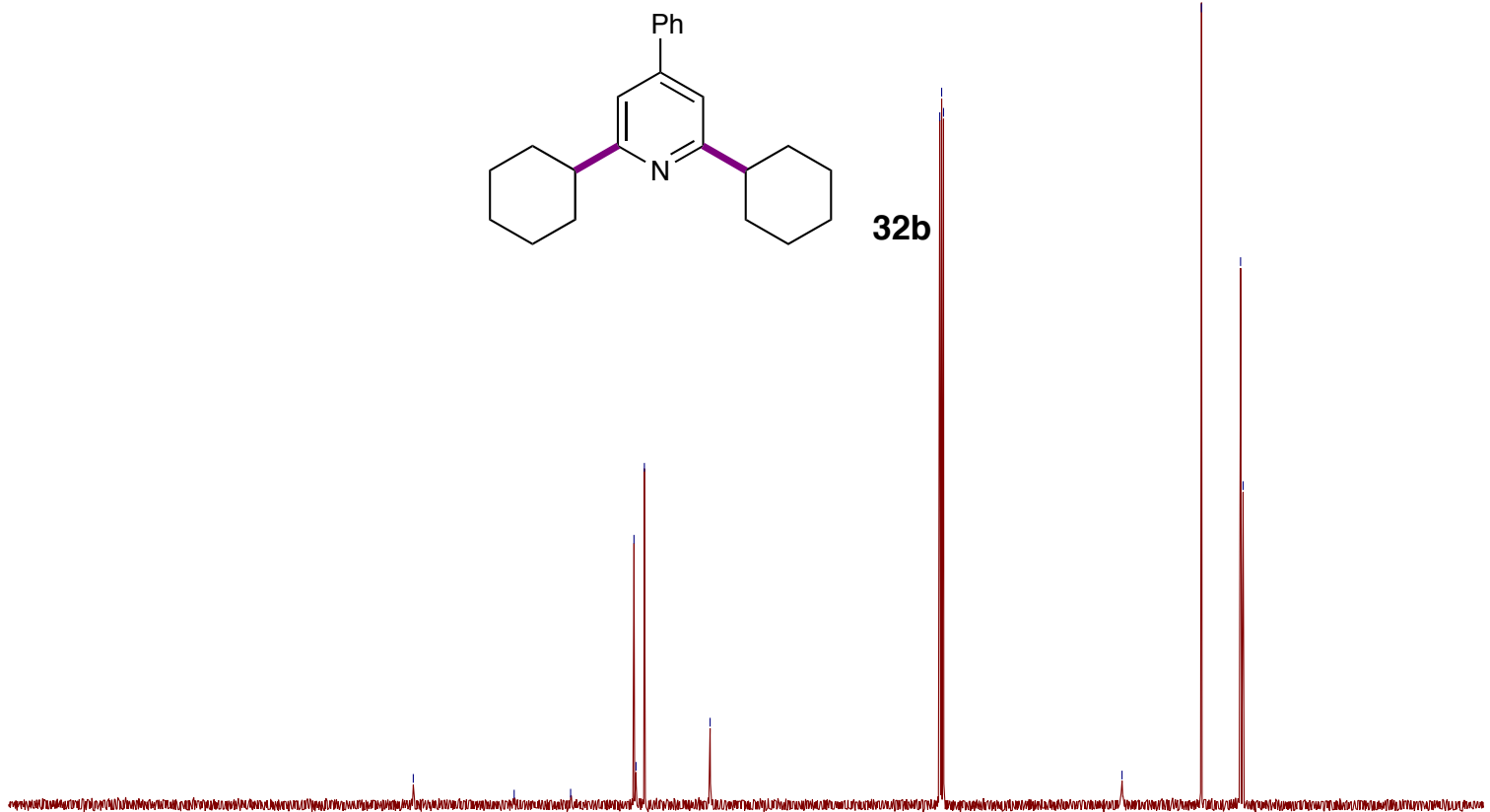

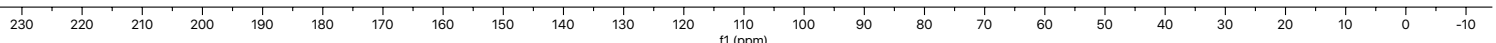




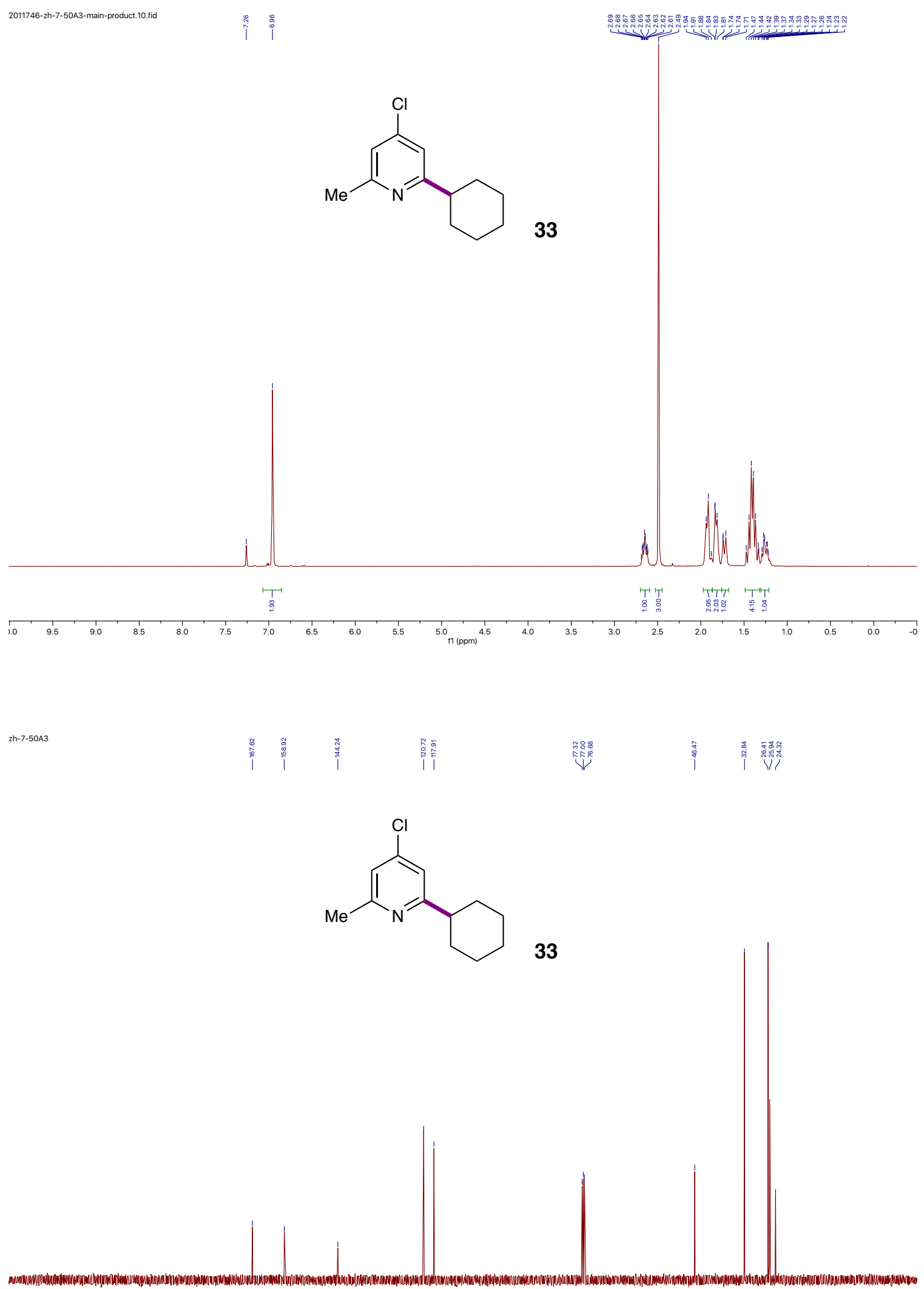

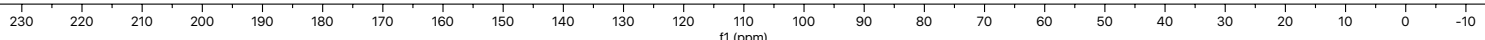




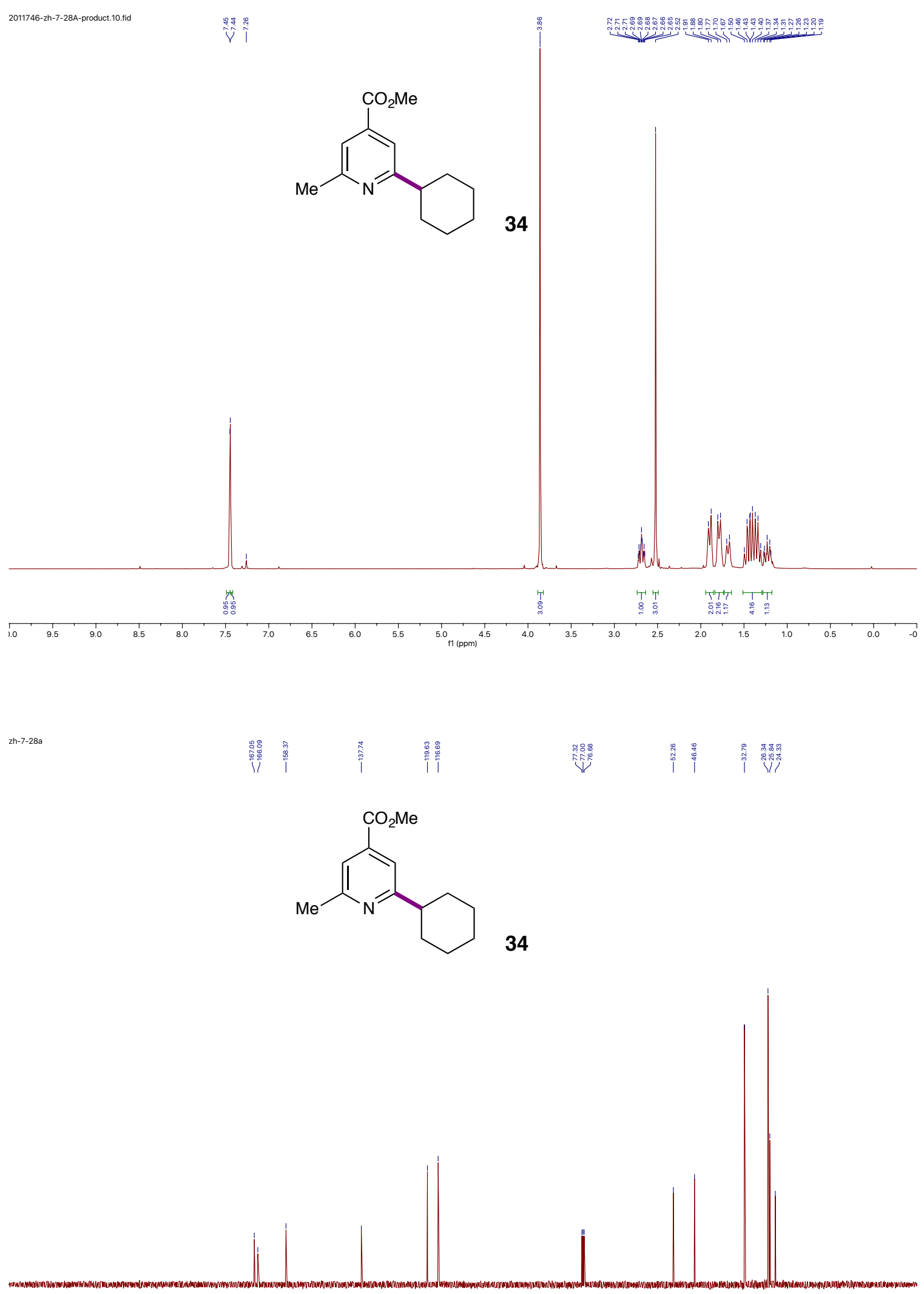

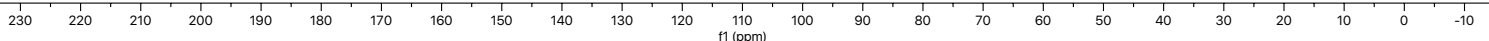




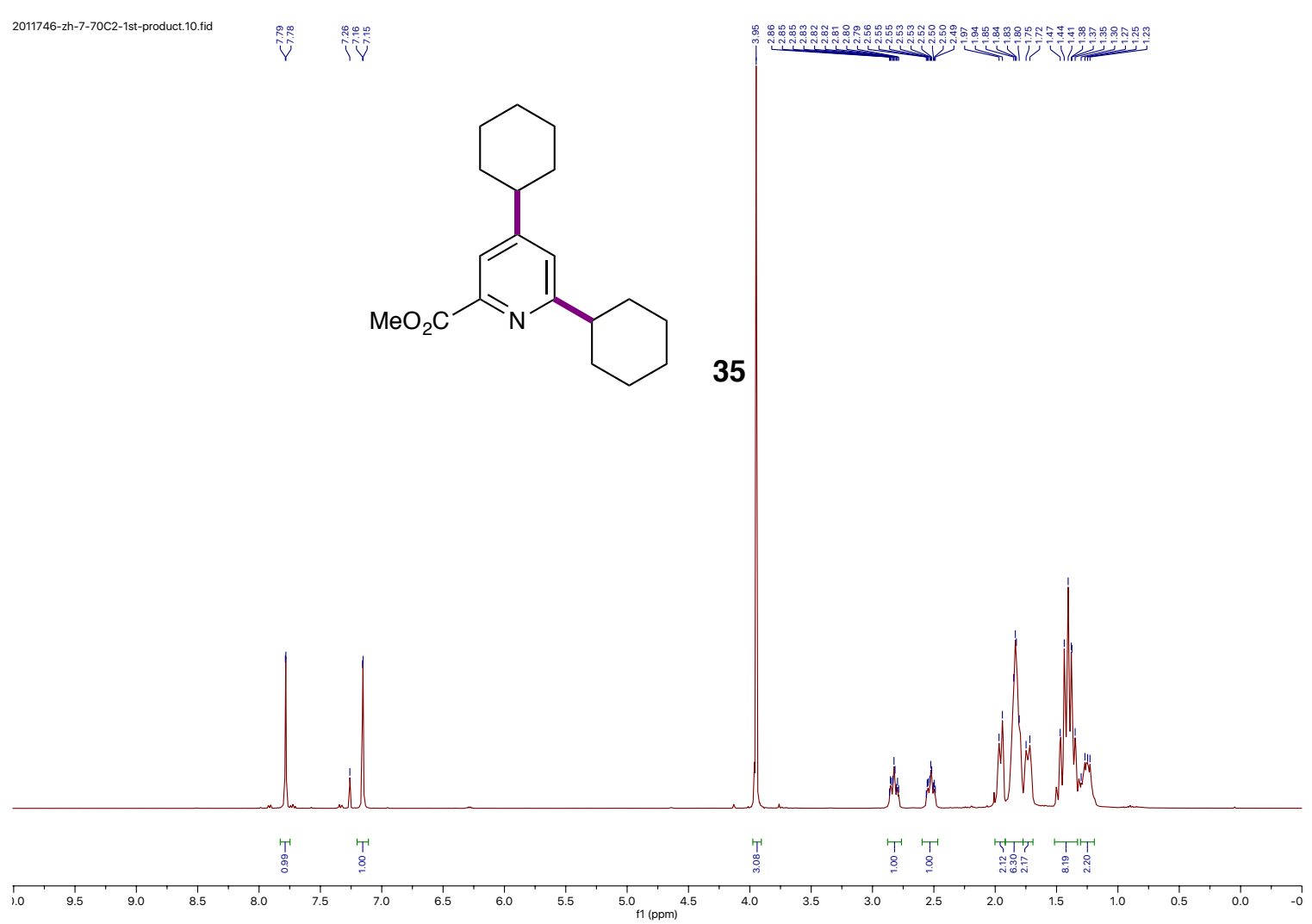

zh7-70c2
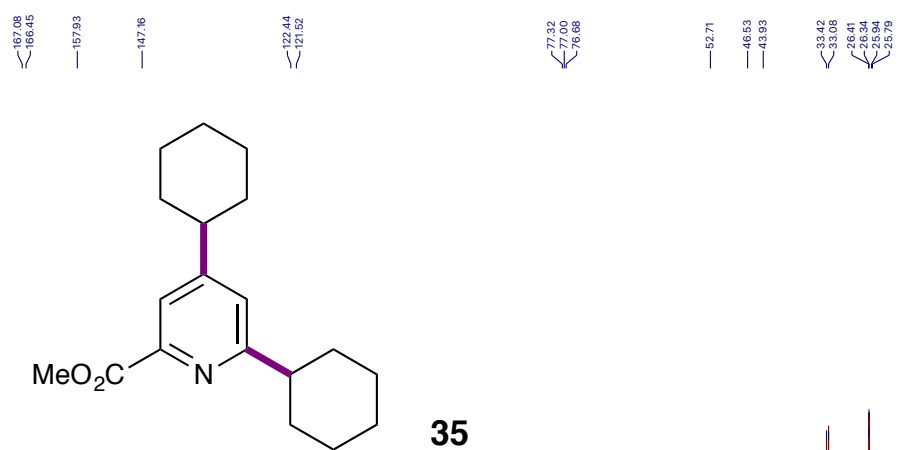

35

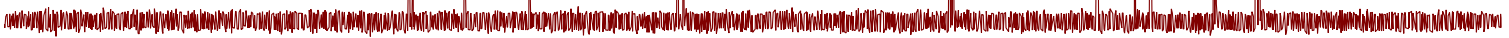

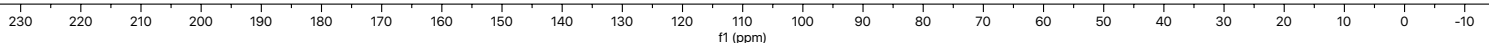




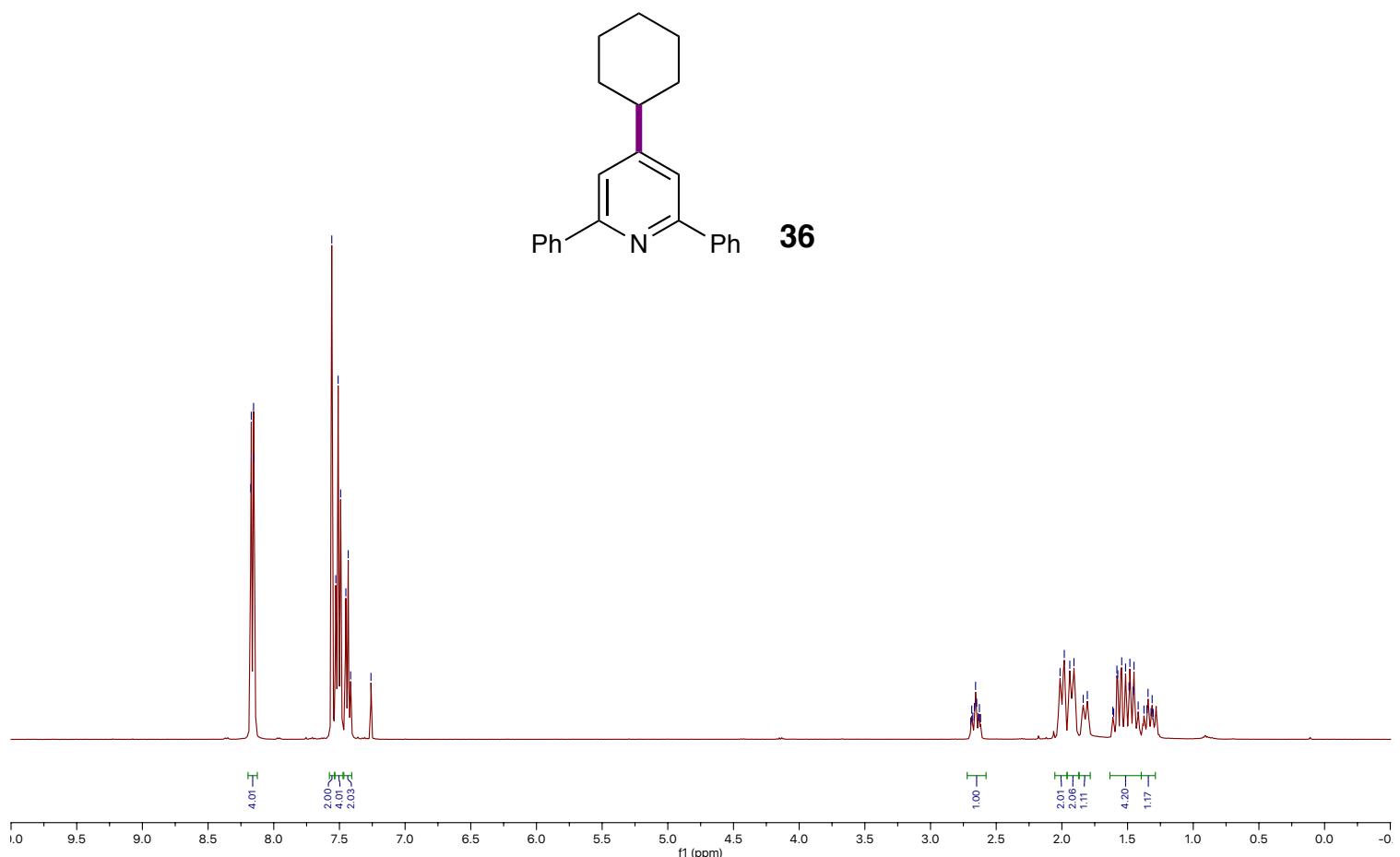

$-2 h-9-28 B 3$

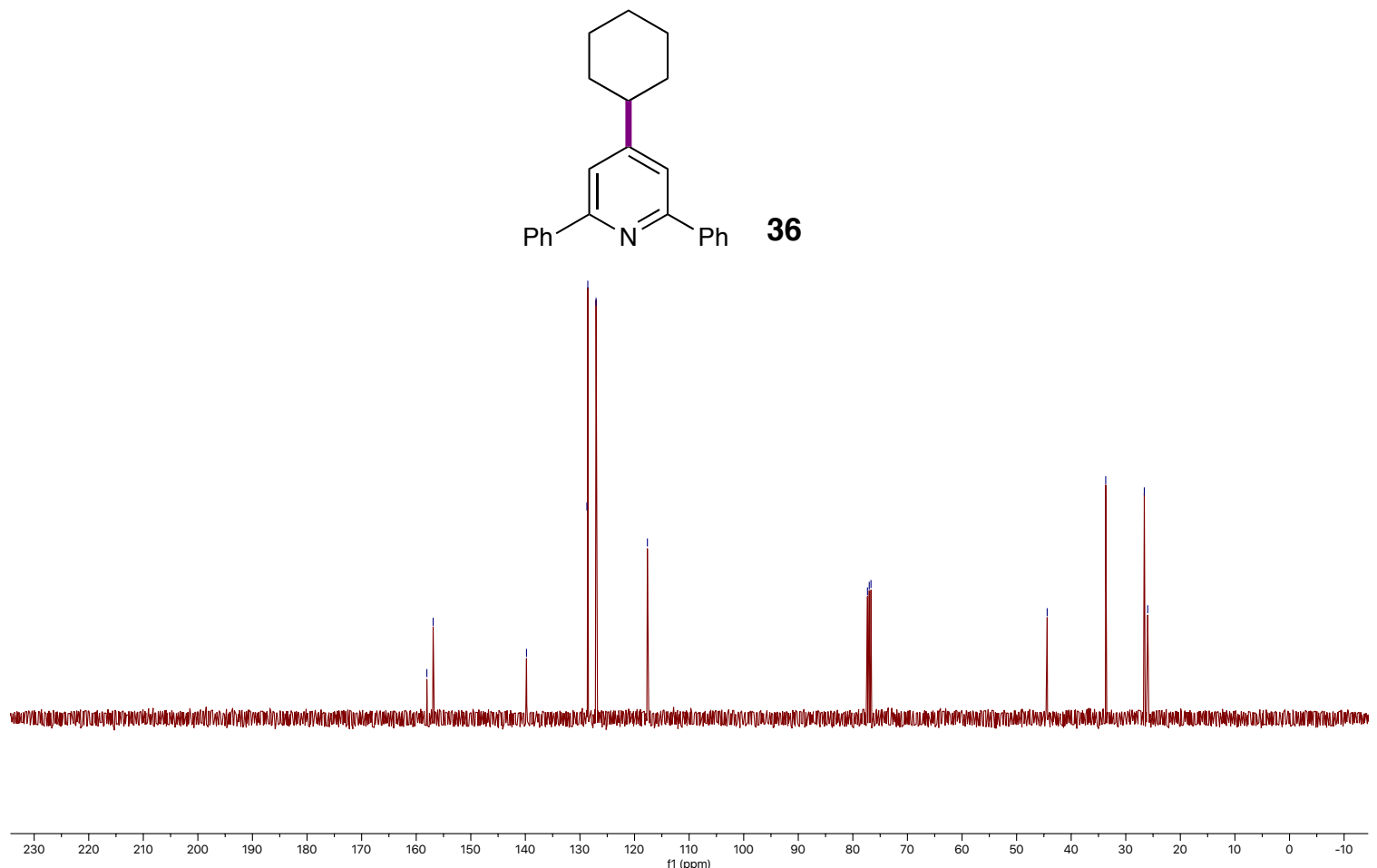




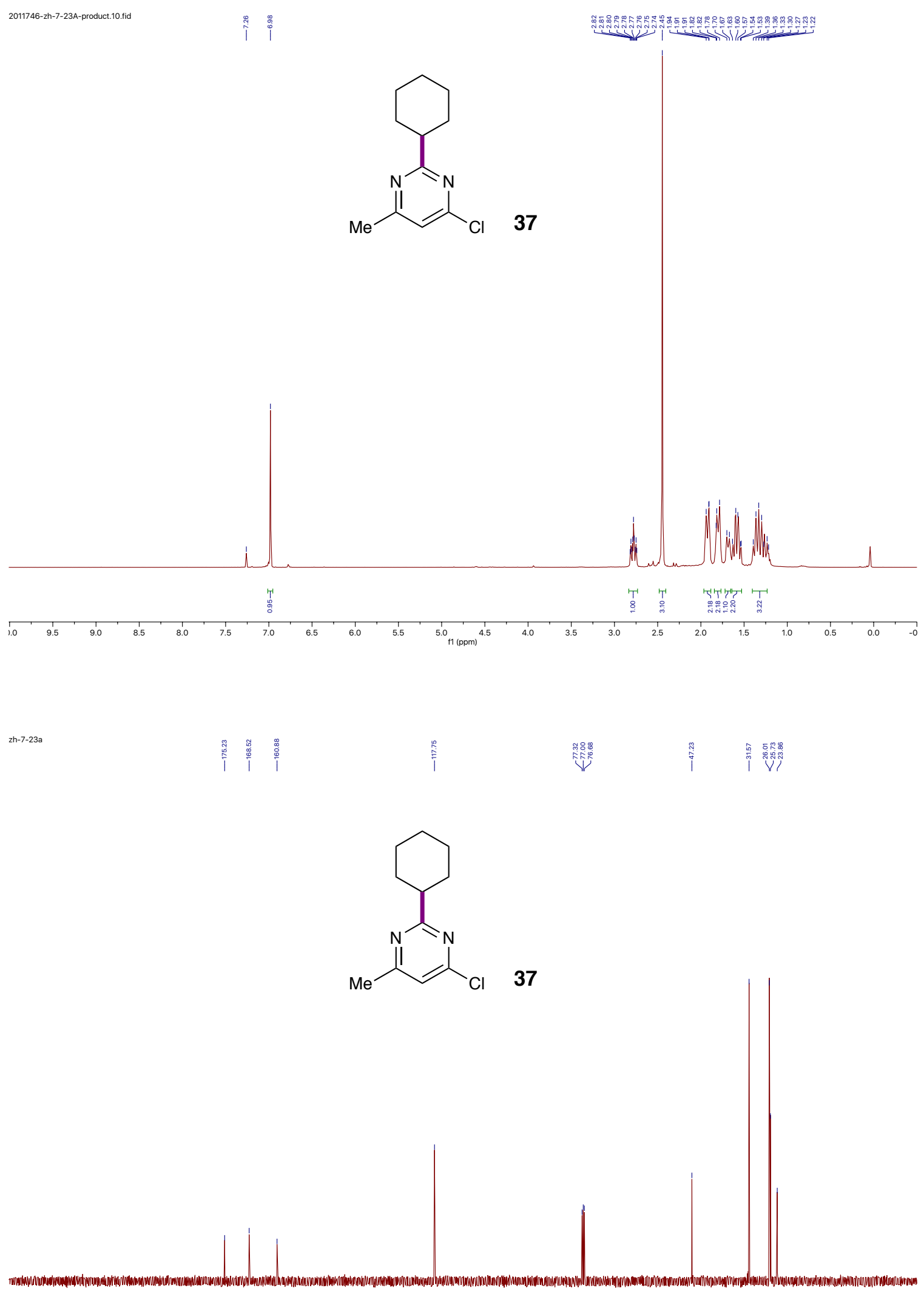

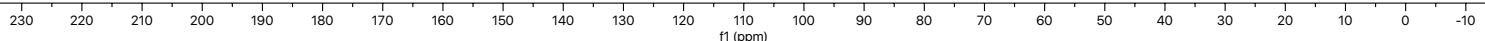




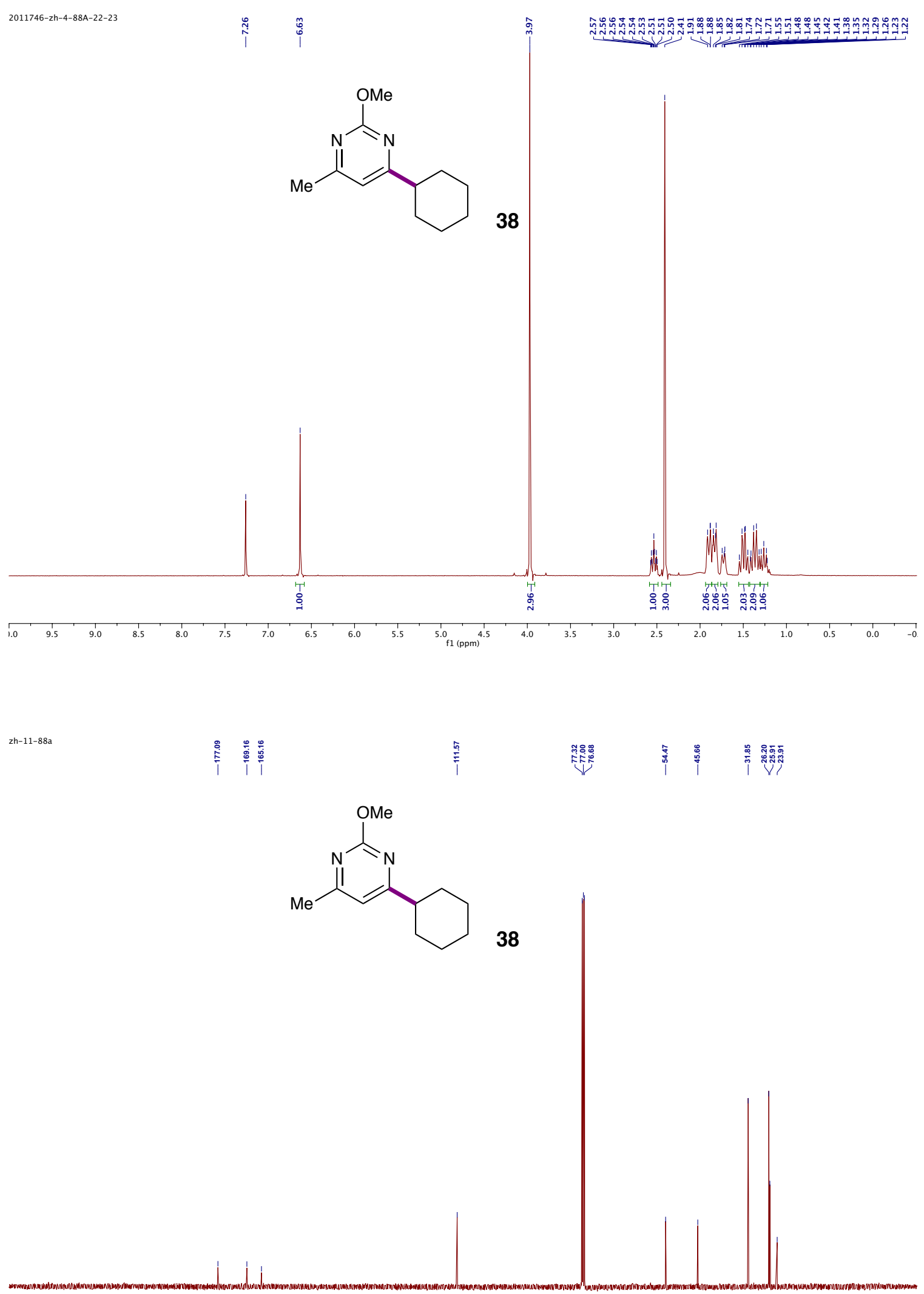

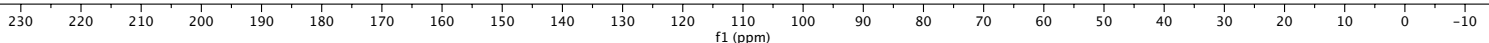




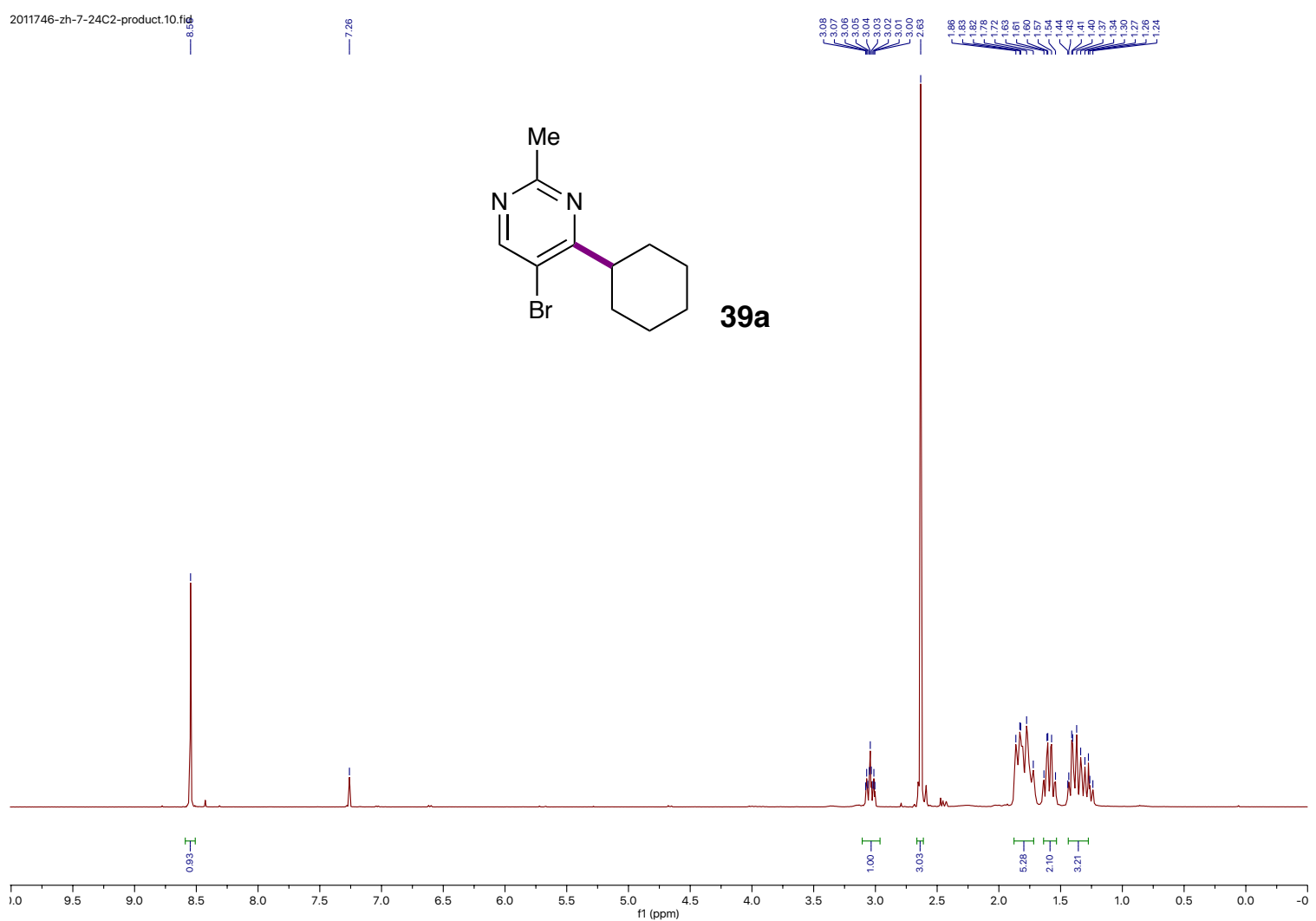

zh-7-24c2

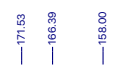

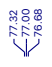
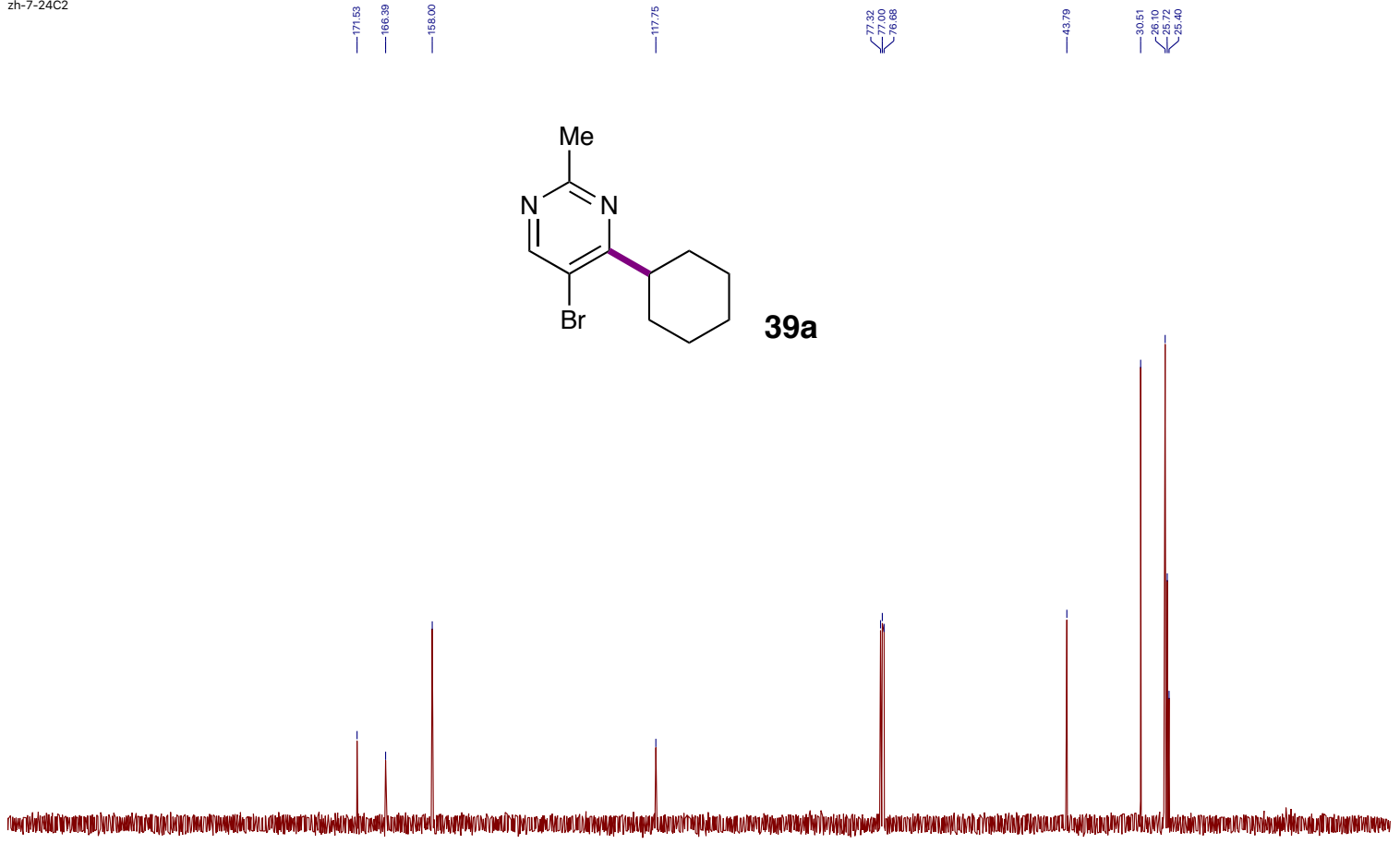

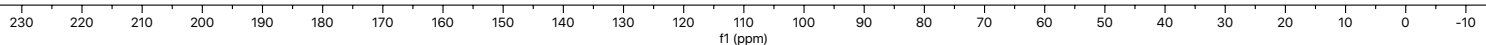




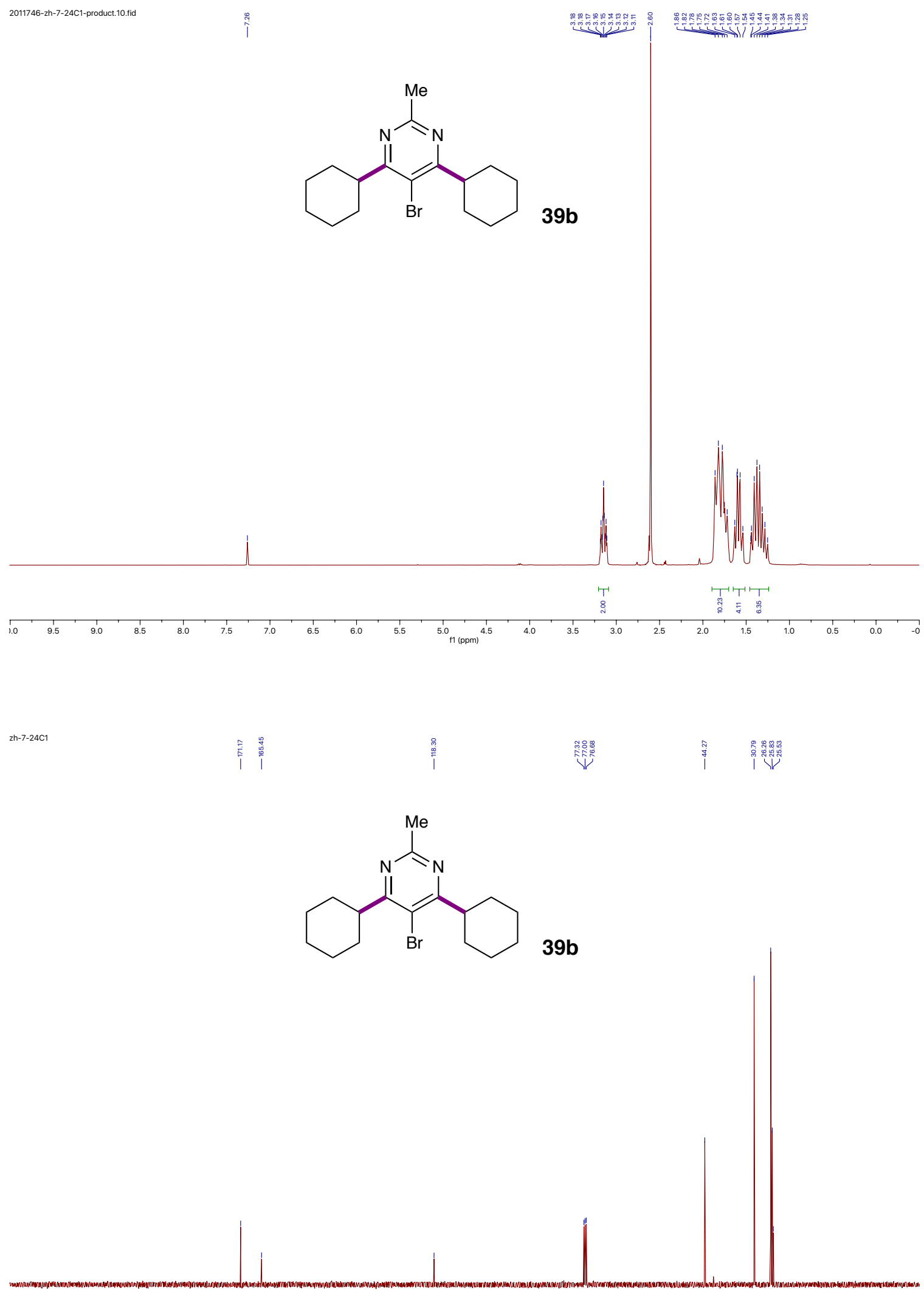

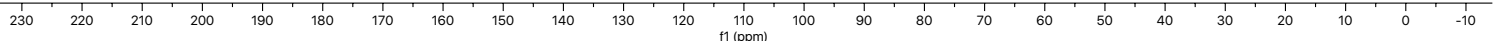




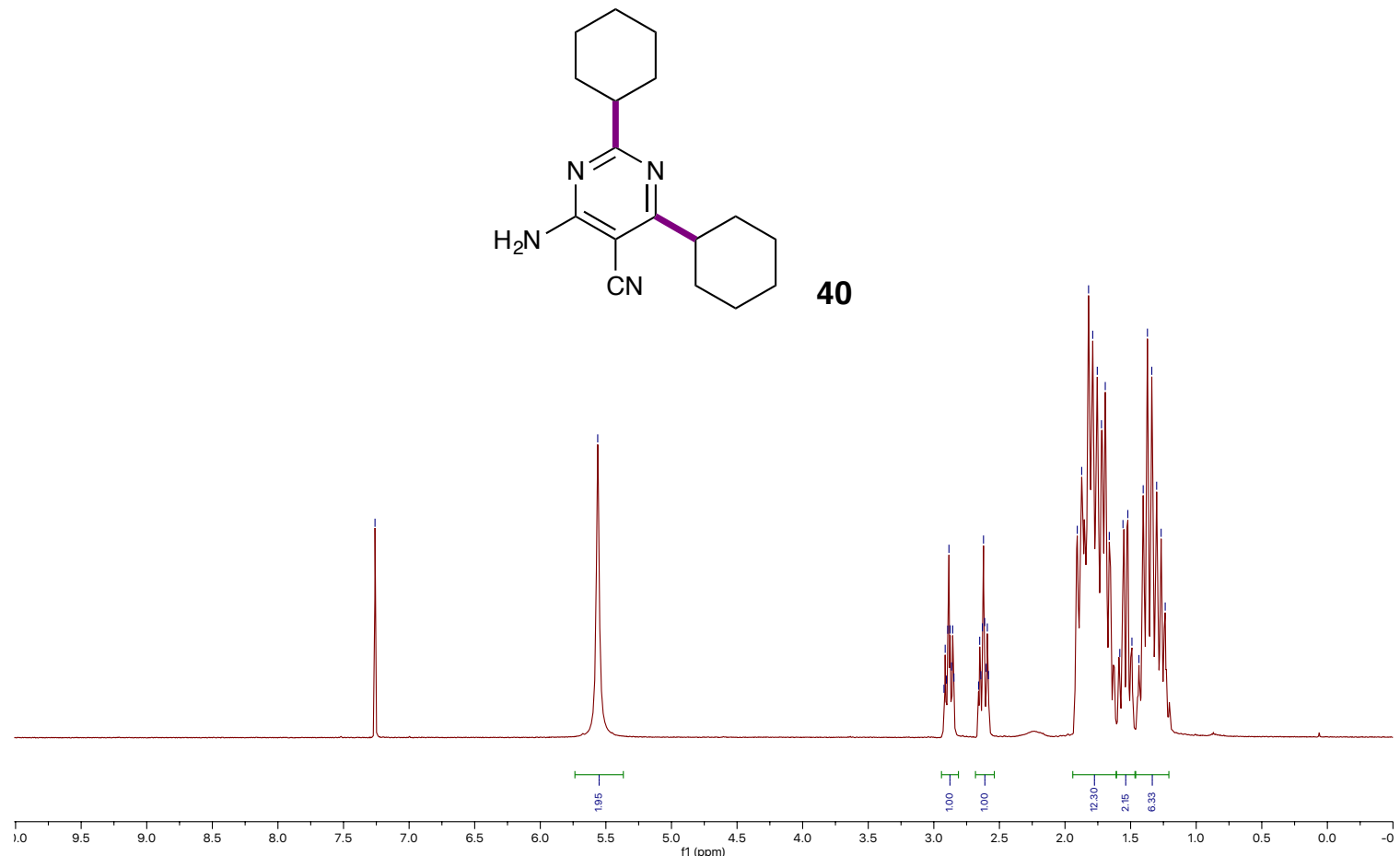

2h-8-102A

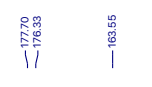

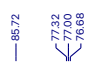
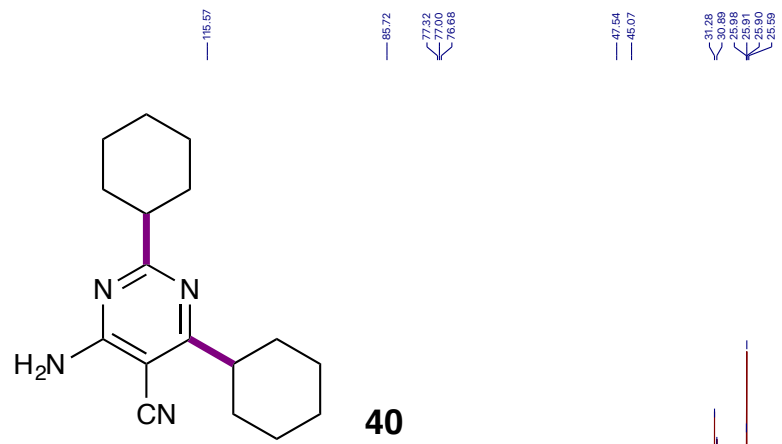

40

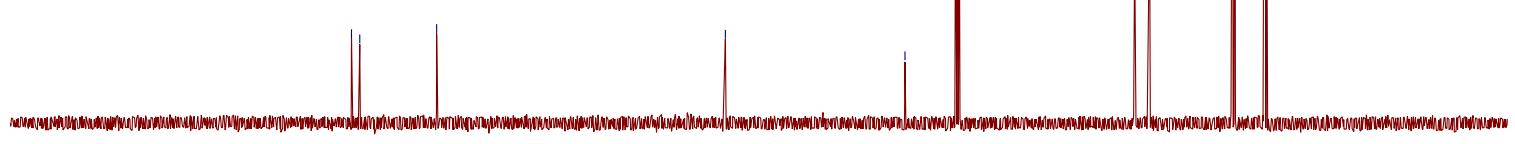

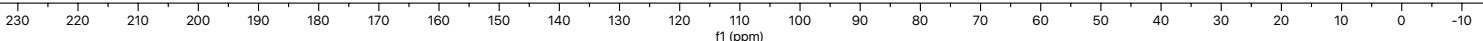




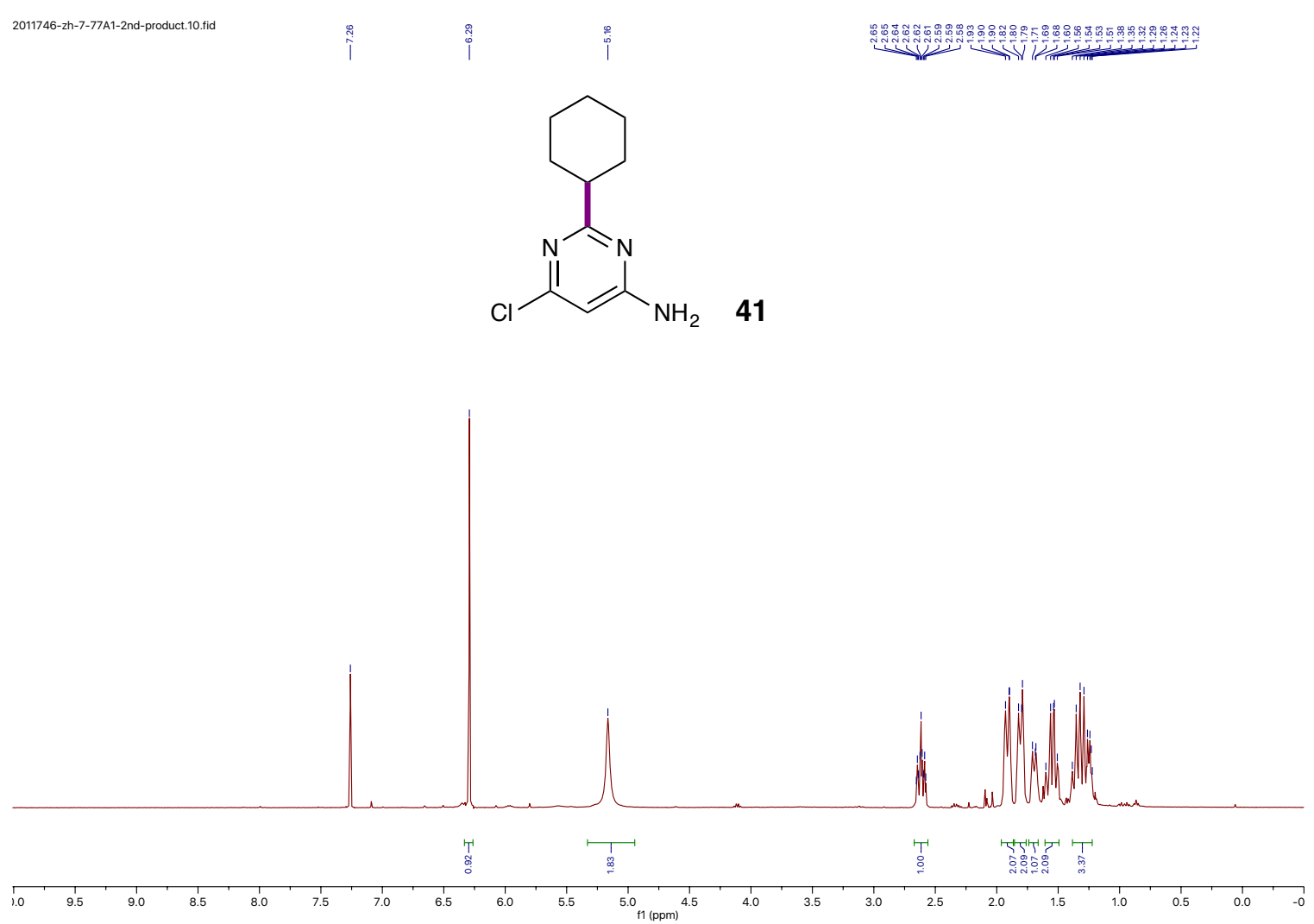

zh7-77A
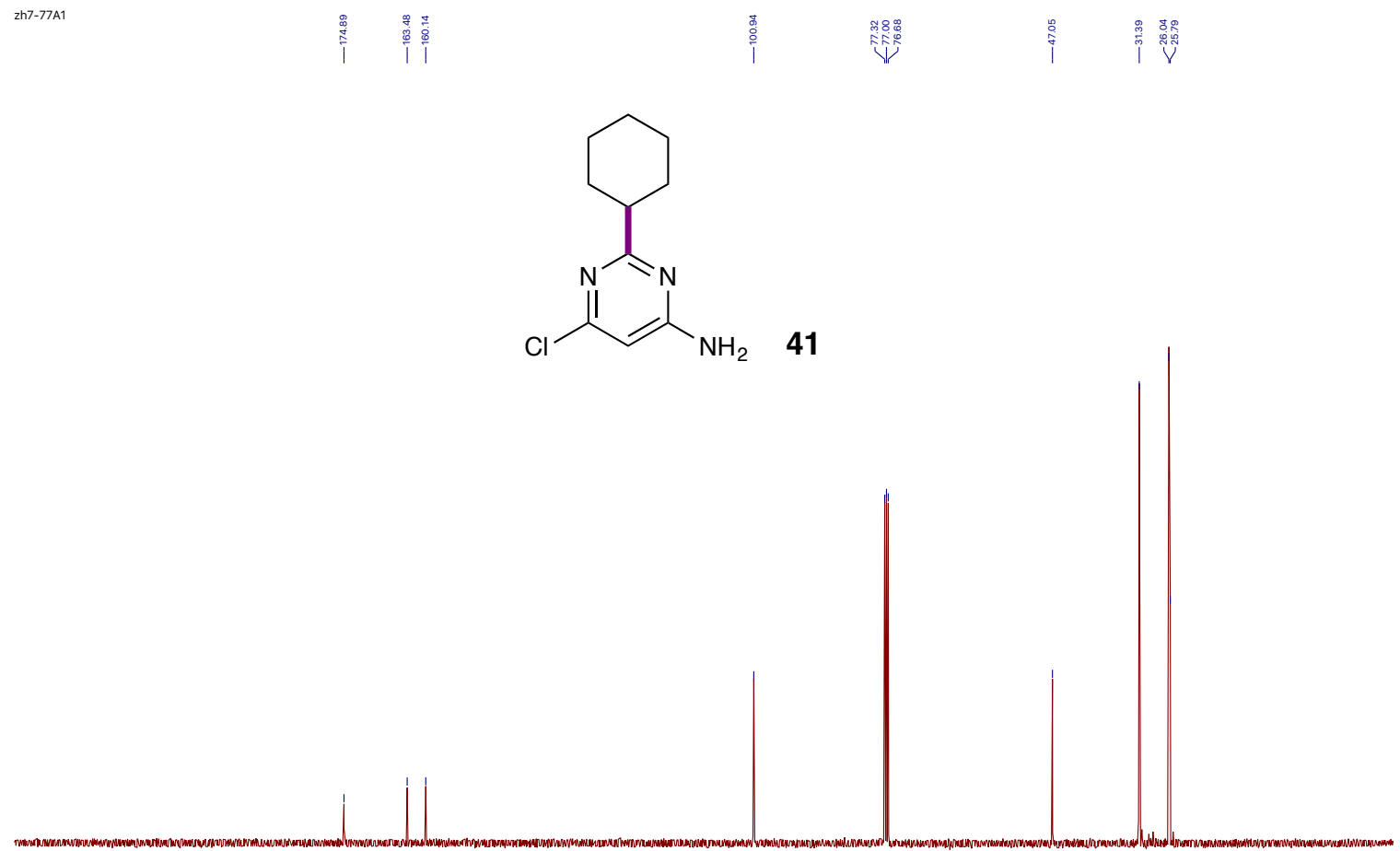

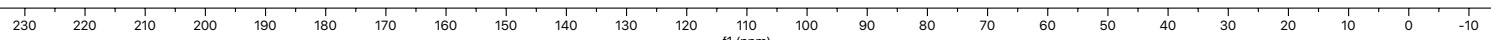



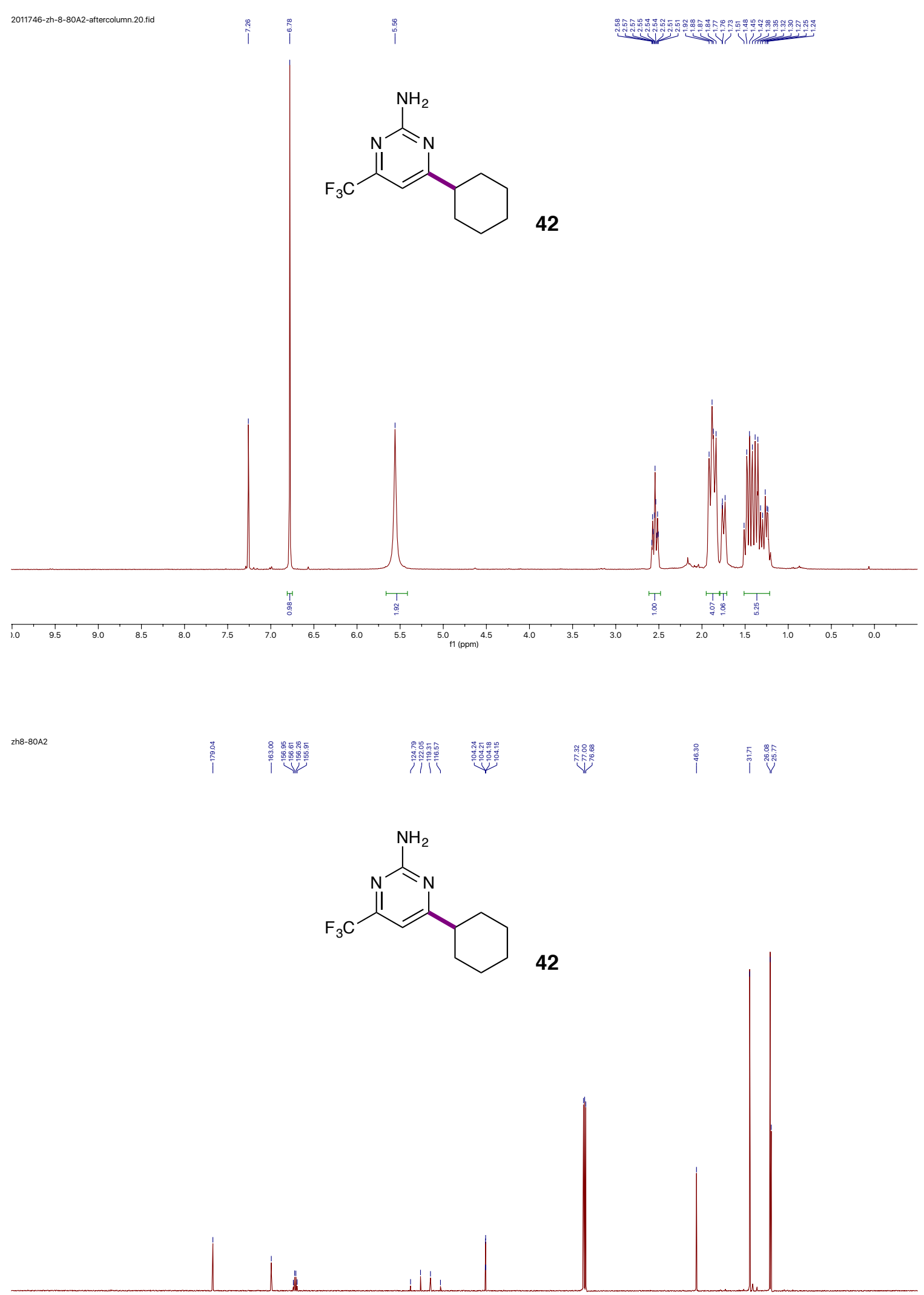

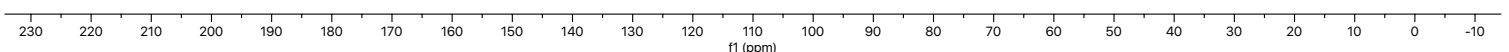




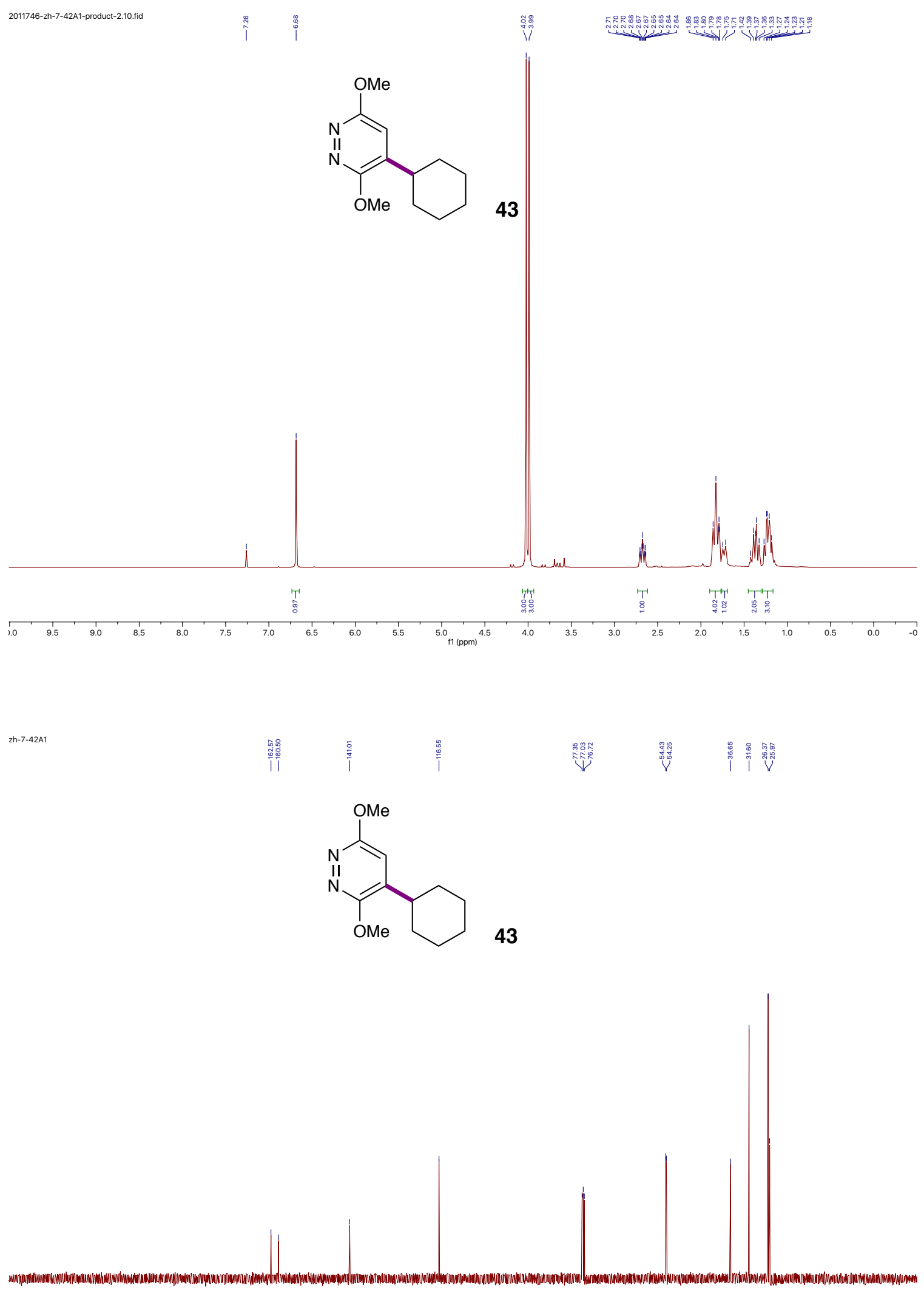

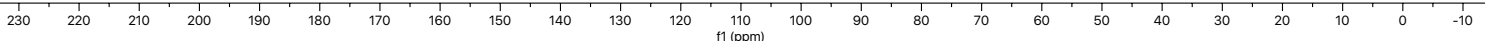



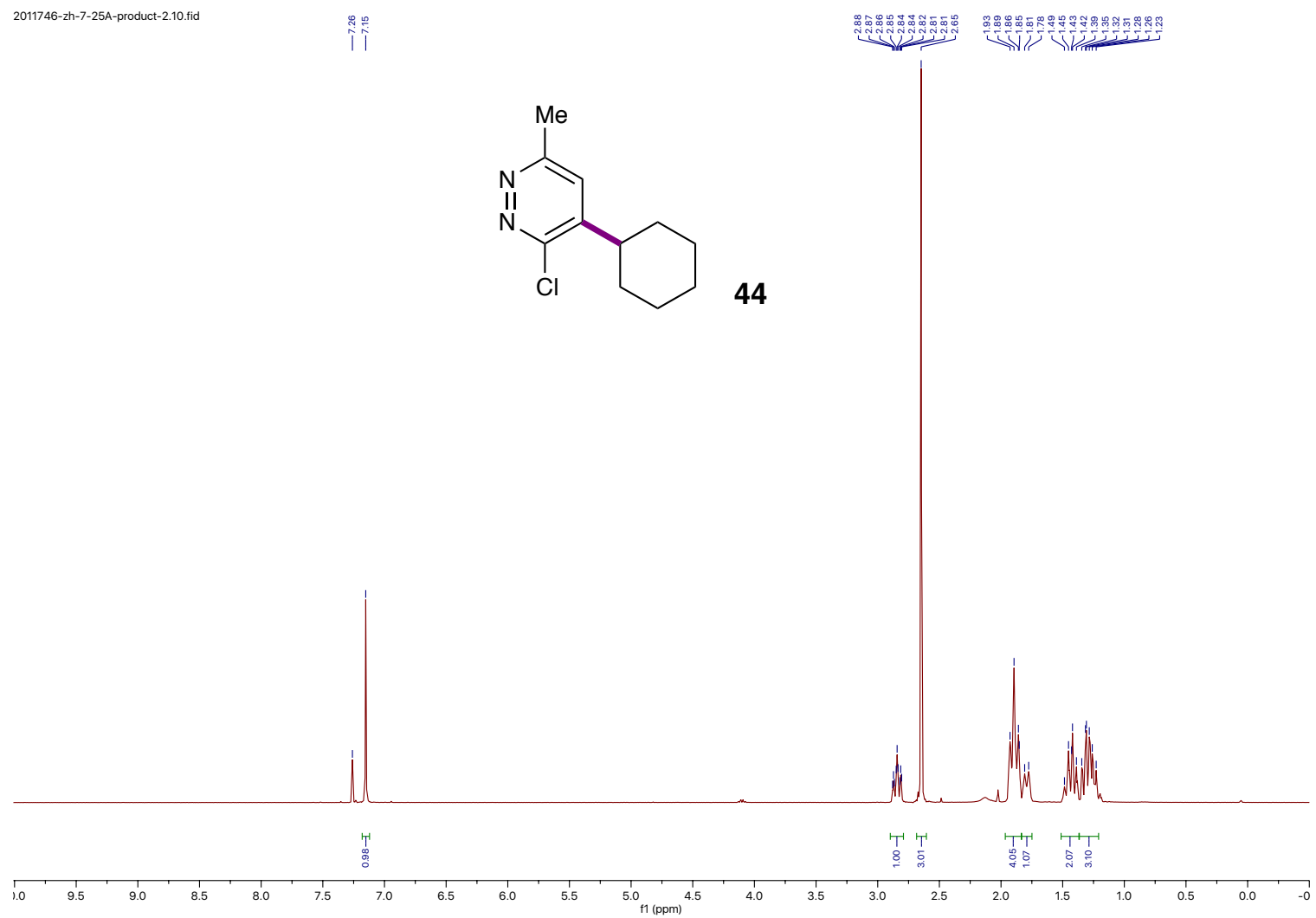

2h-7-25a

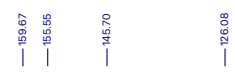

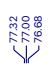

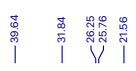

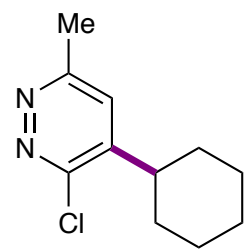

44

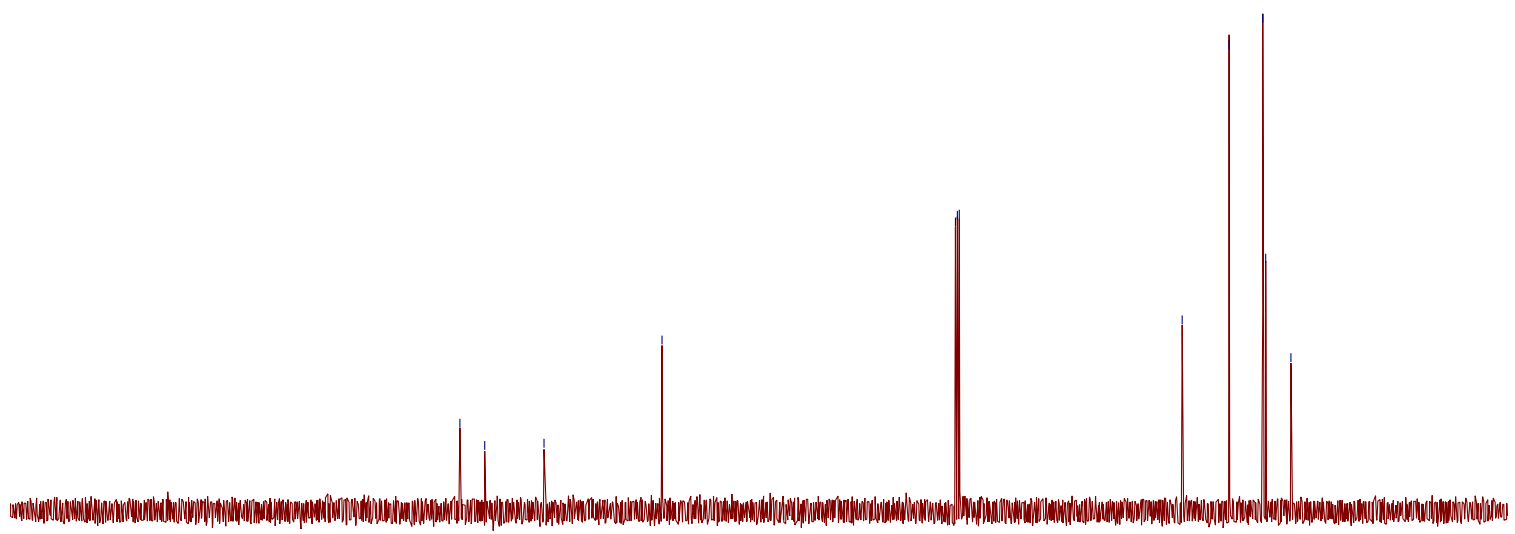

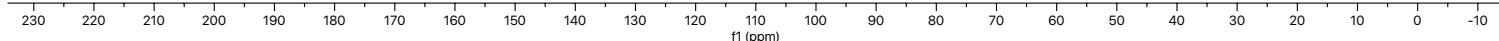



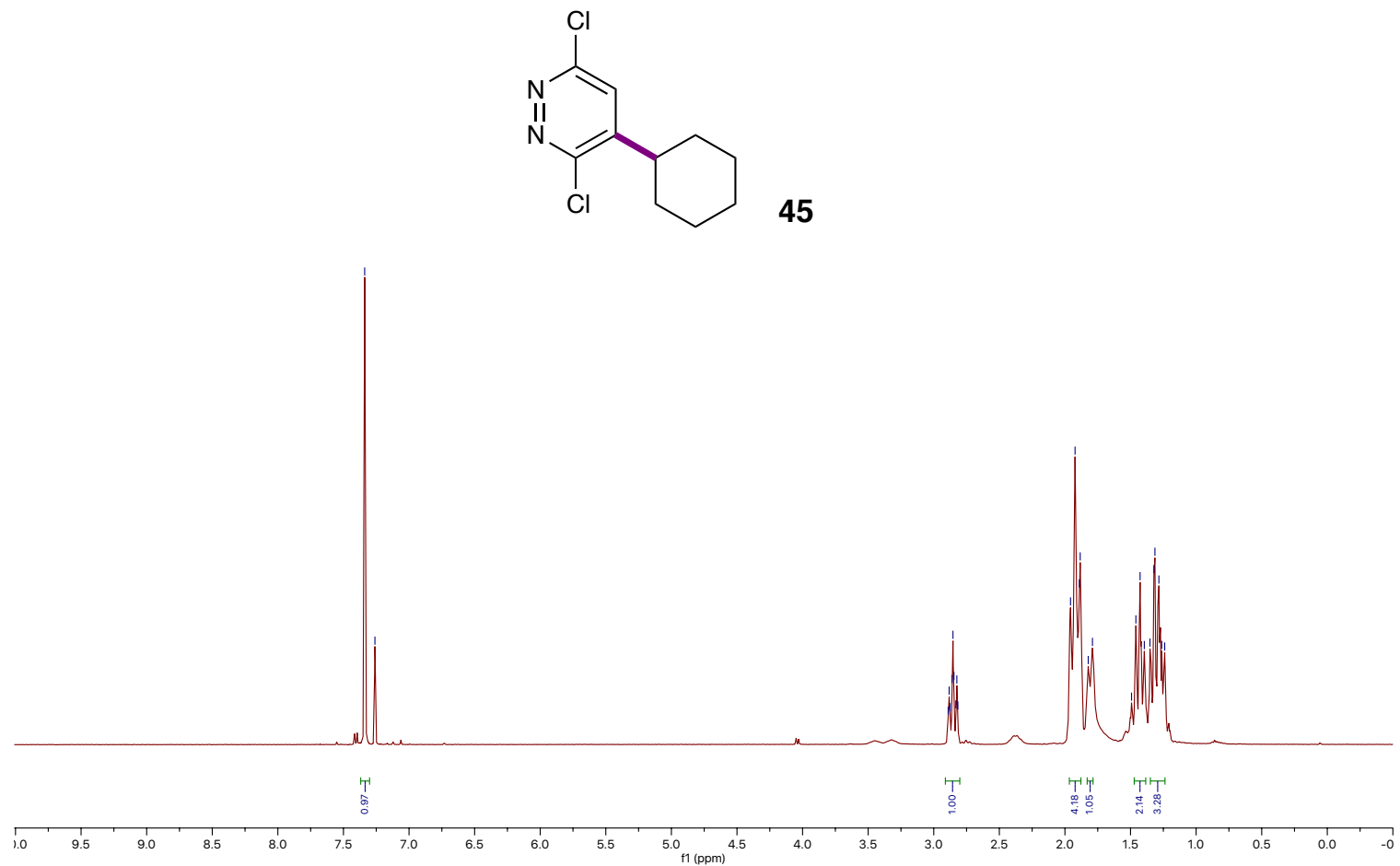

zh-7-43a

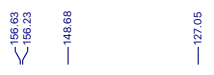

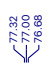

।

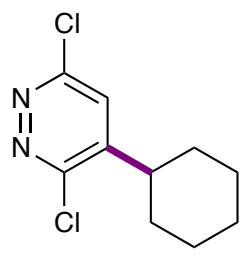

45

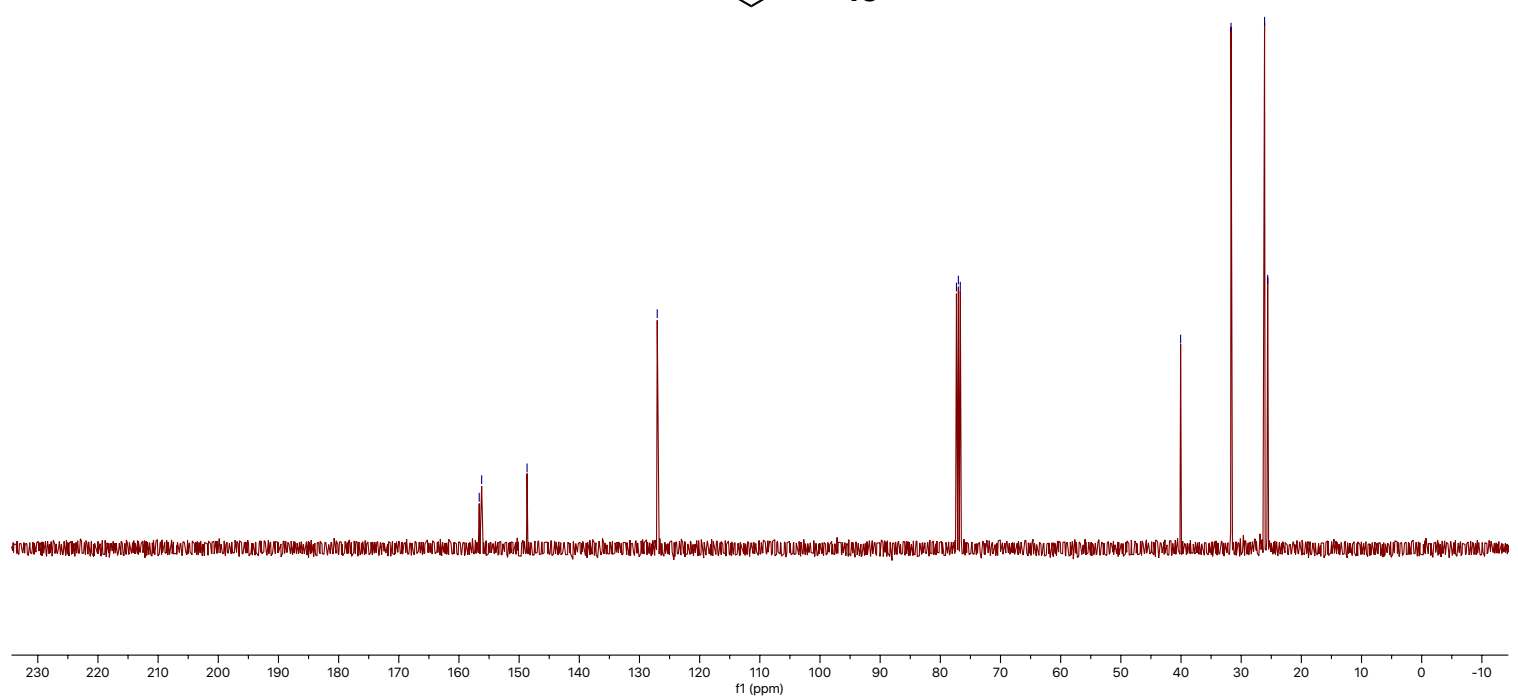



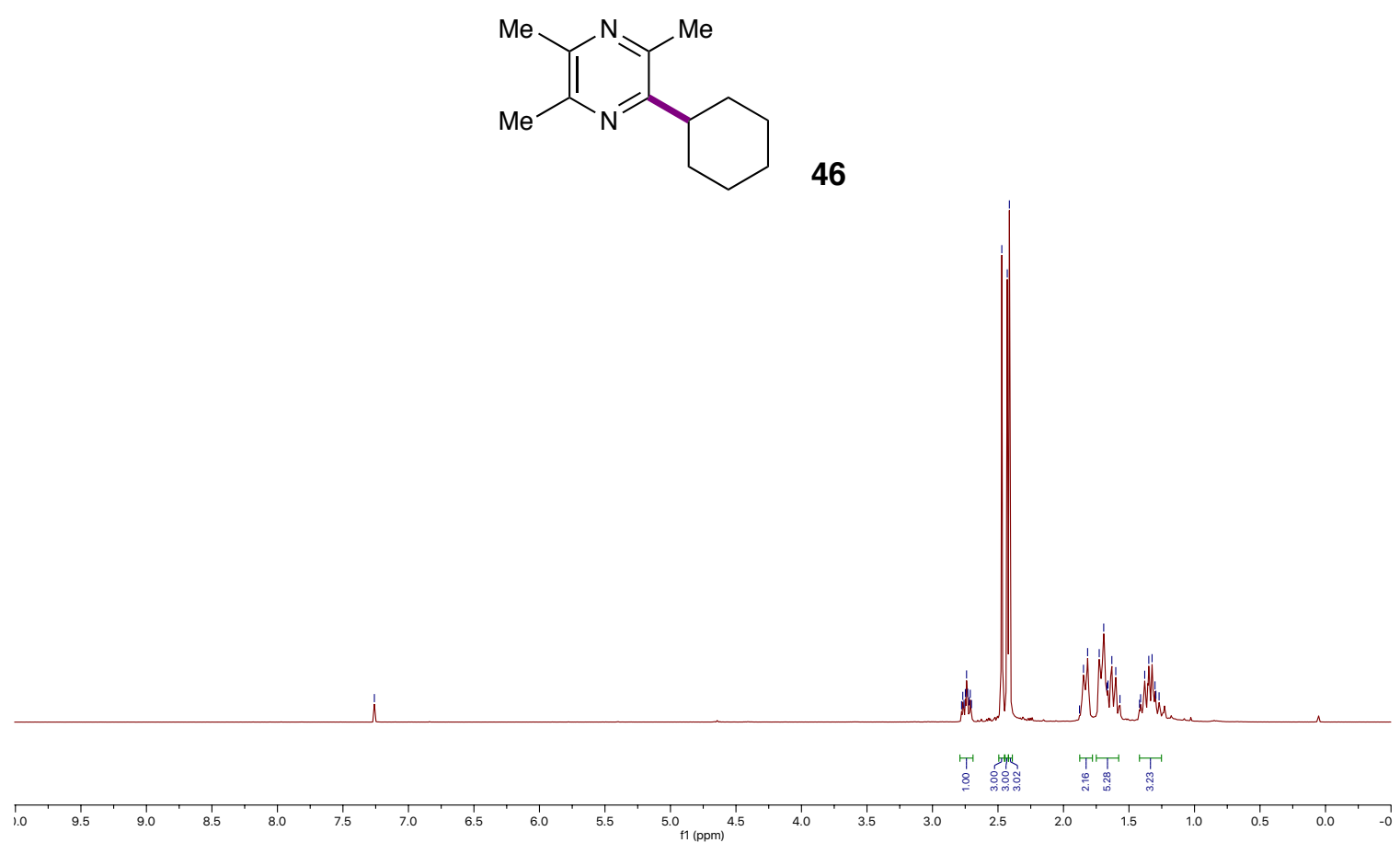

zh-7-26a
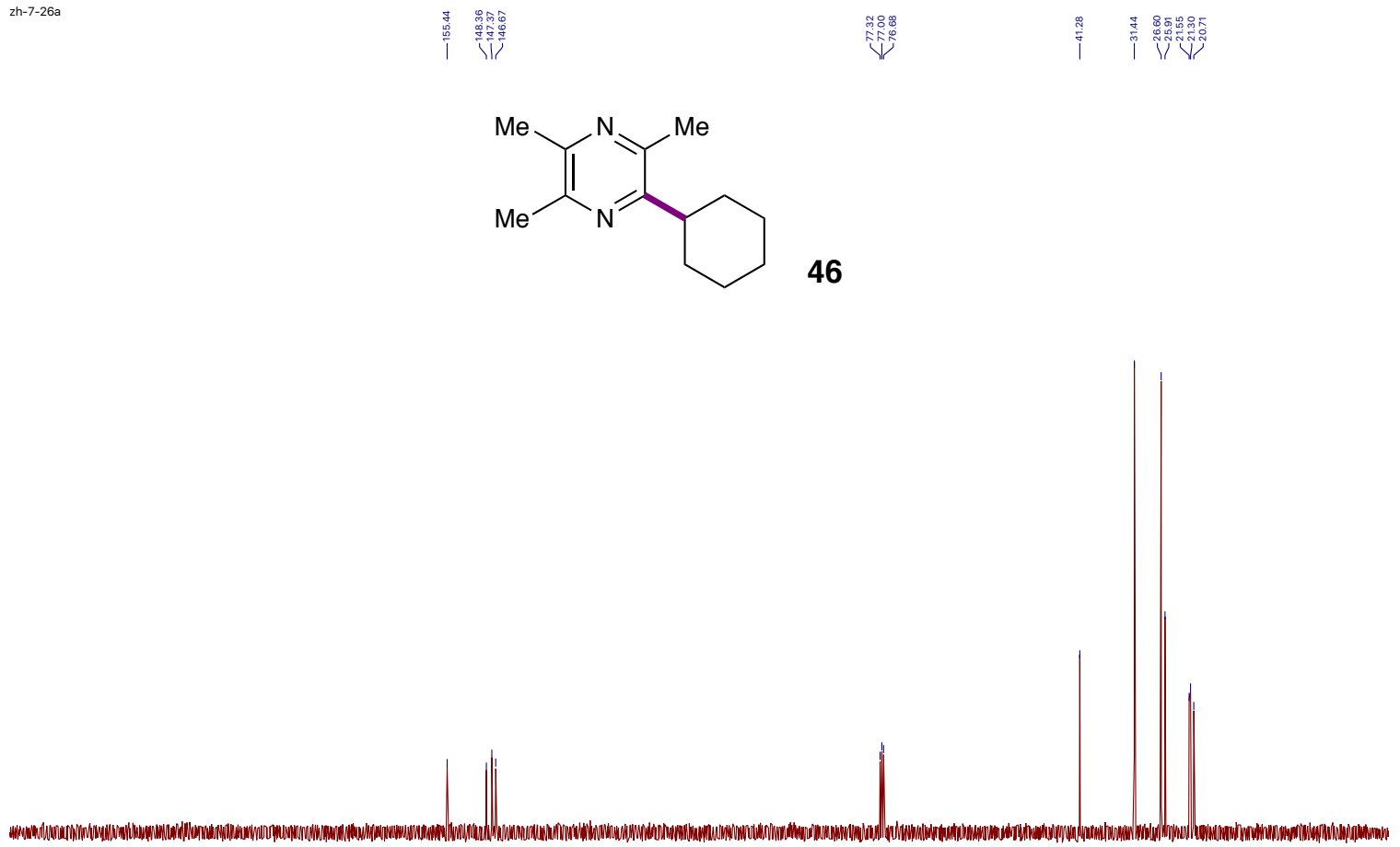

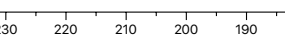

$\underbrace{110}_{\mathrm{f1}(\mathrm{ppm})} 10$ 


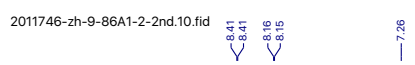
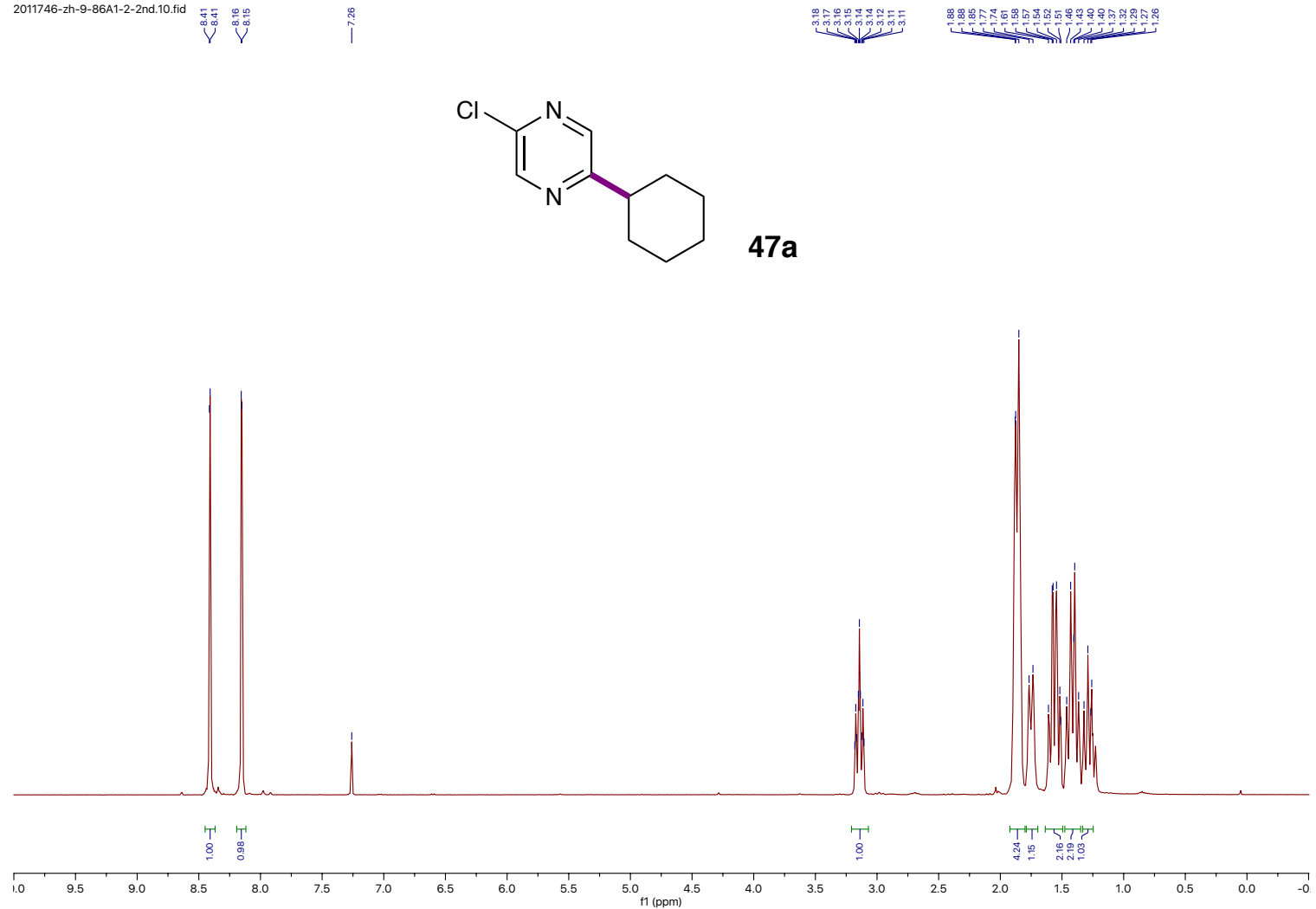

zh-9-86A-2nd
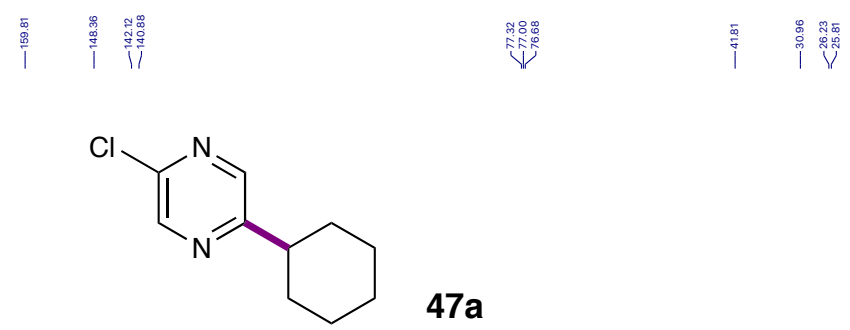

$47 a$

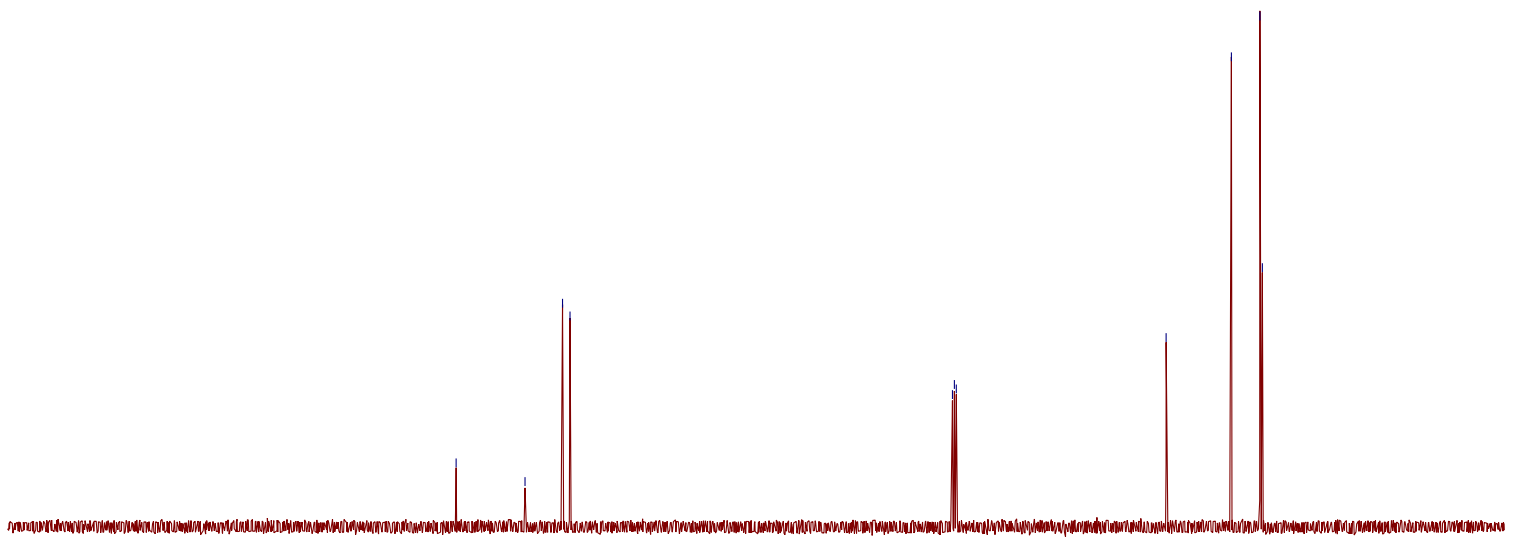

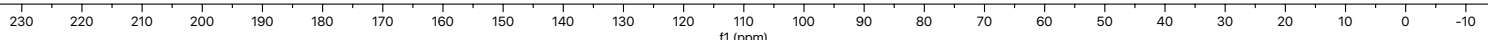


<smiles>Clc1ncc(C2CCCCC2)nc1C1CCCCC1</smiles>

$47 b$

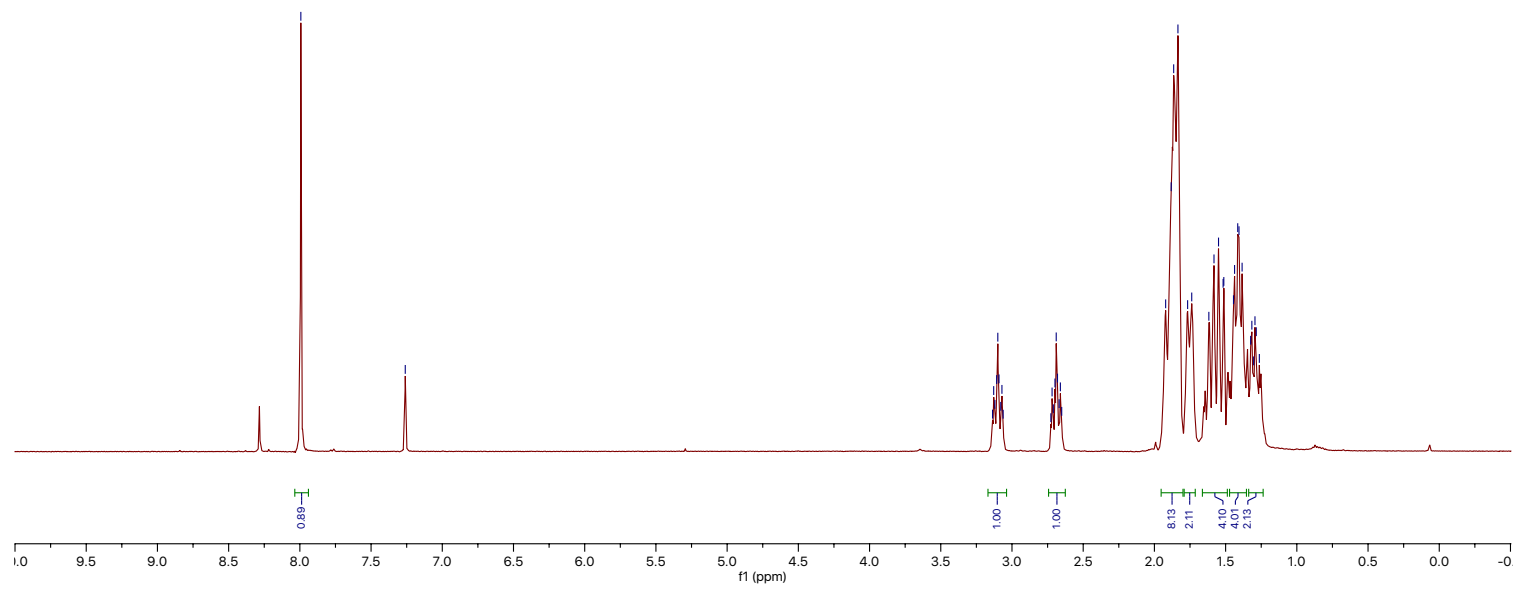

zh-9-86A-1st<smiles>Clc1ncc(C2CCCCC2)nc1C1CCCCC1</smiles>

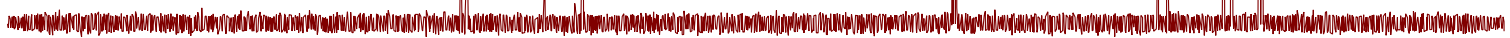

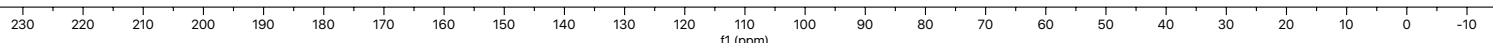



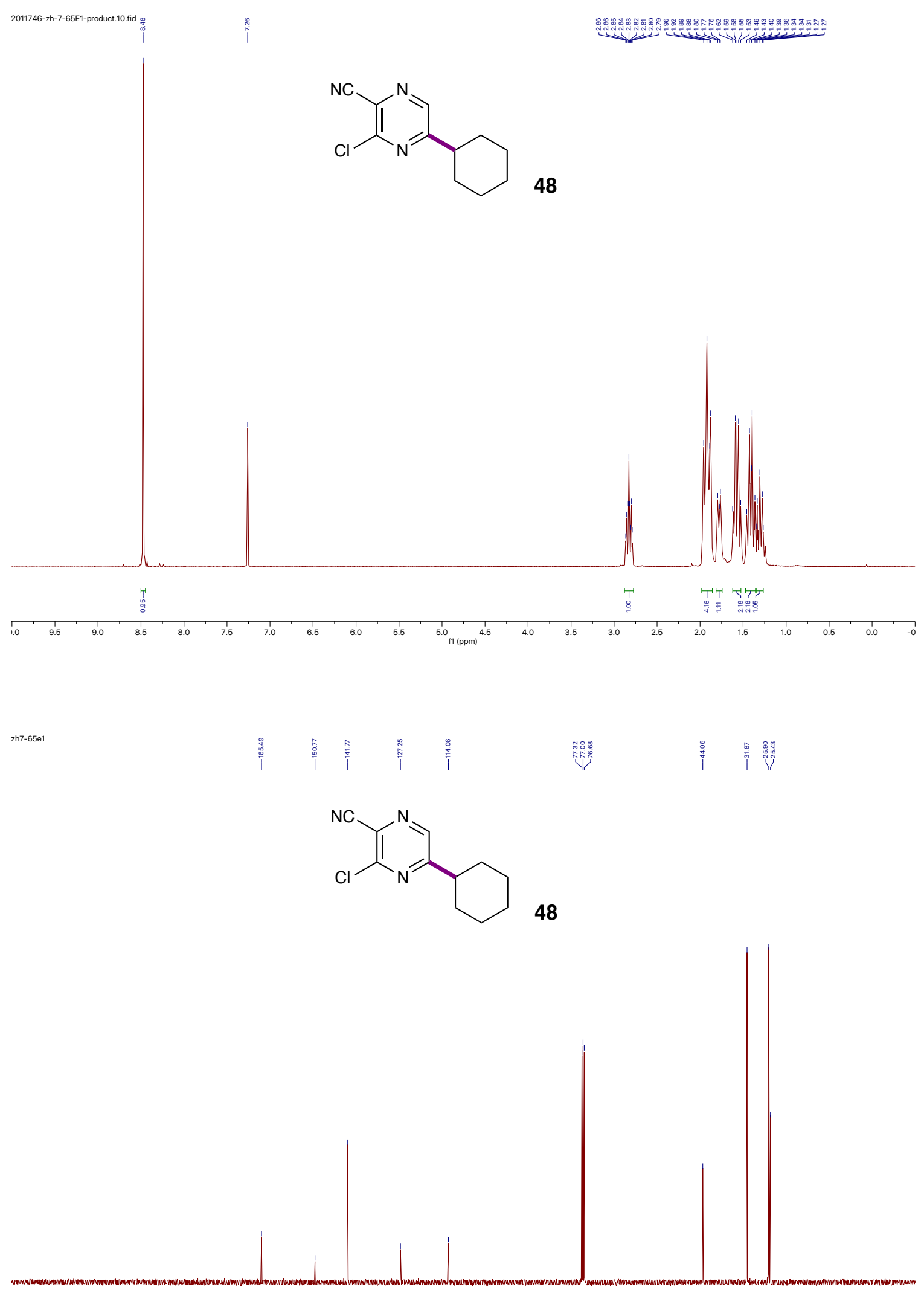

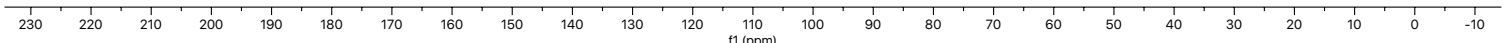



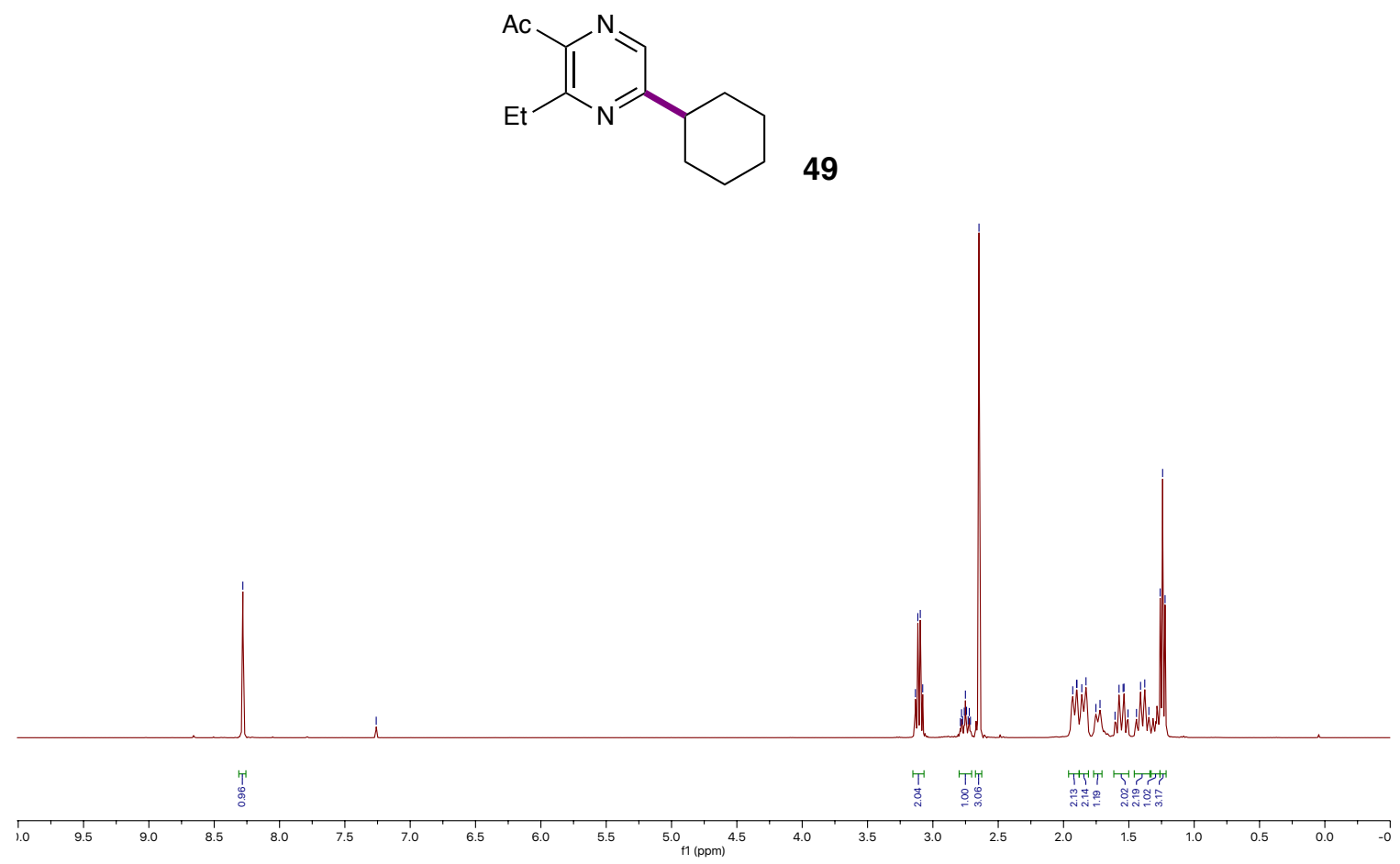

zh-7-27a

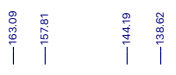

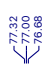
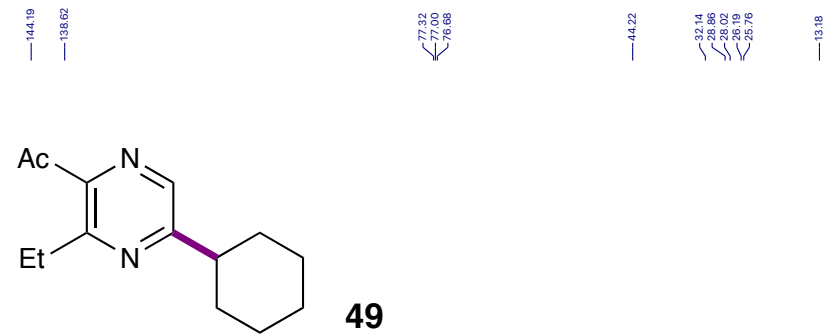

49

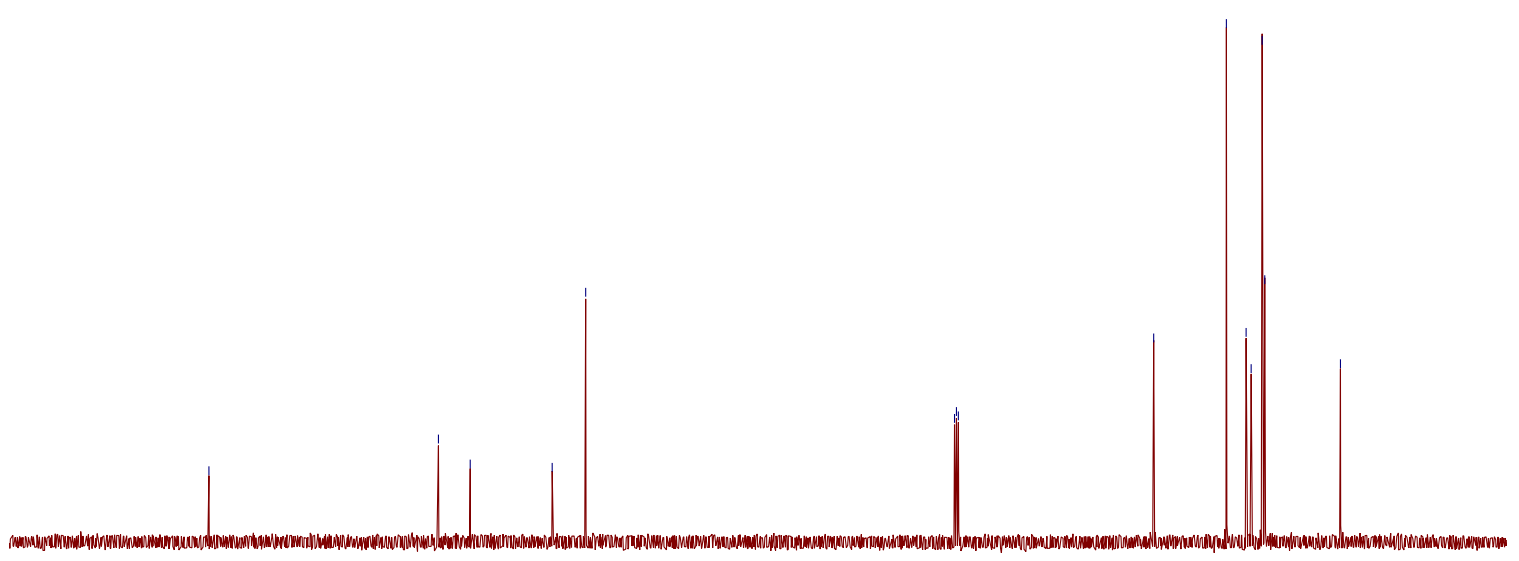

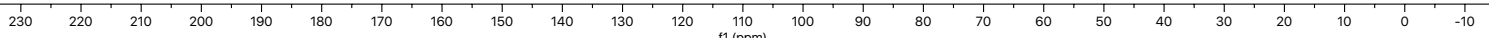




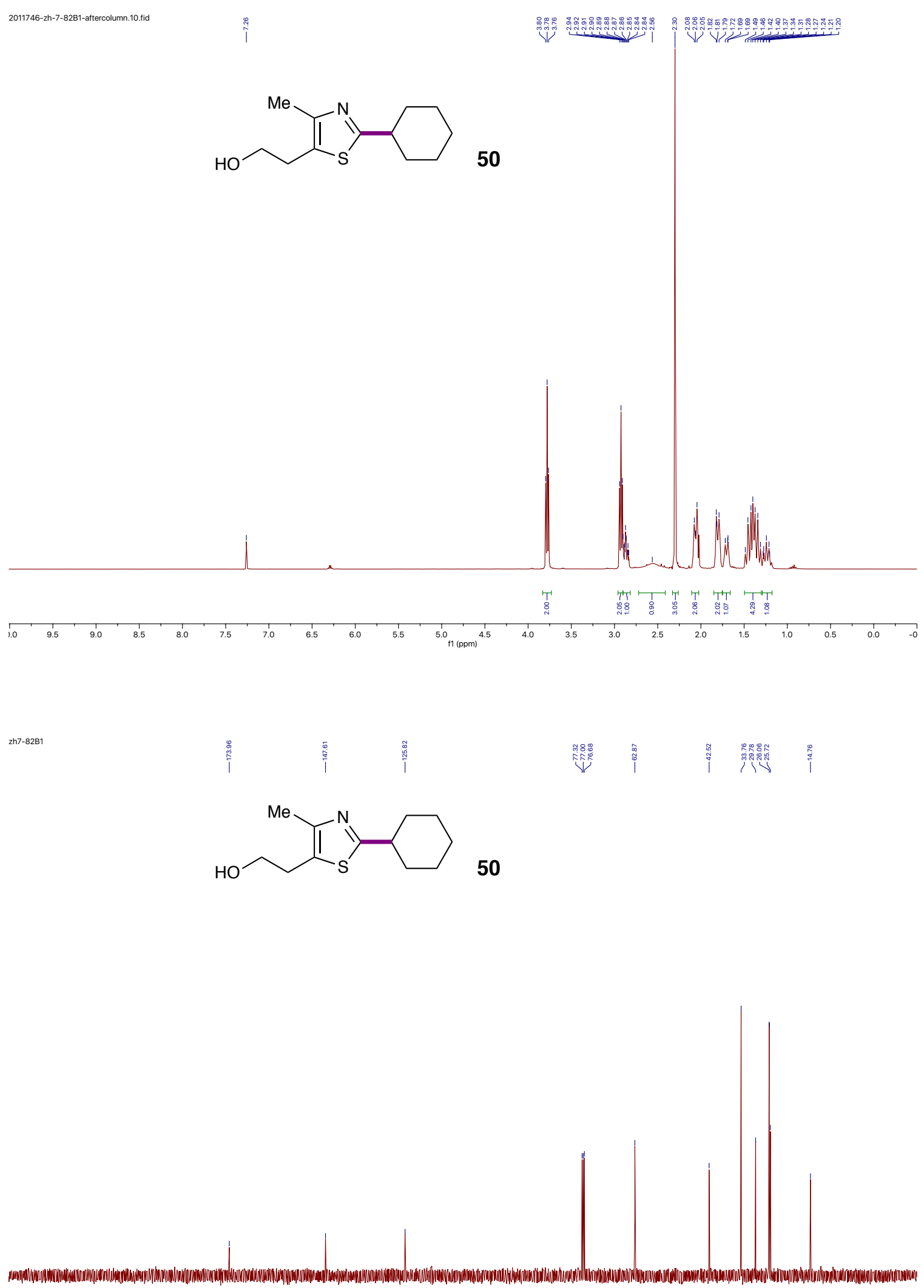

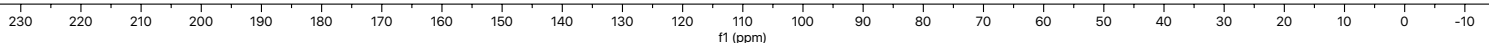



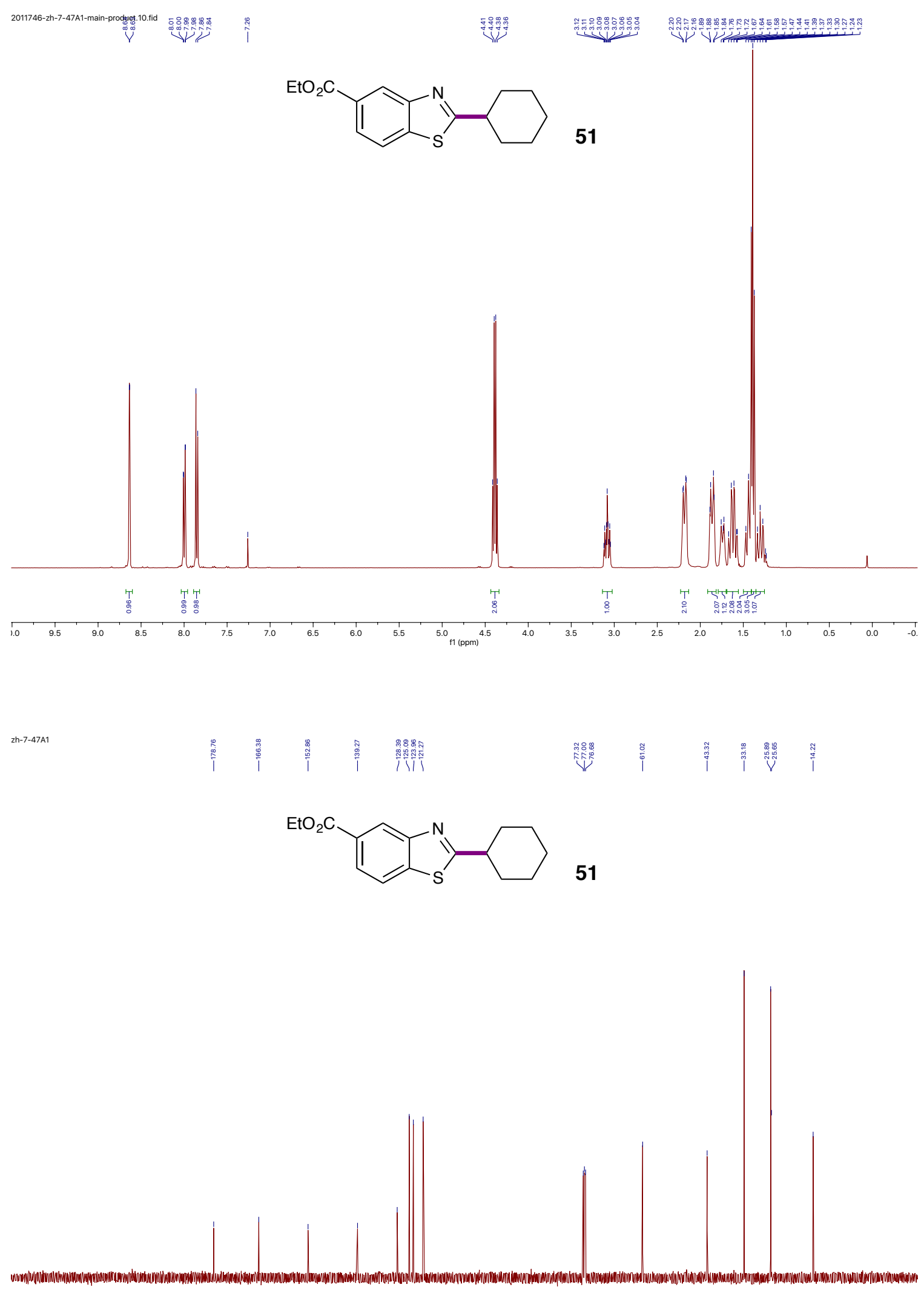

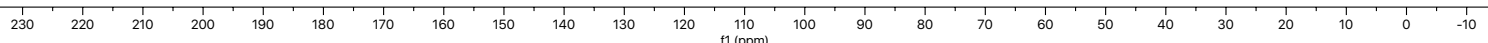




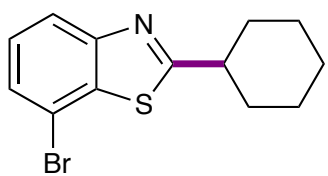

52

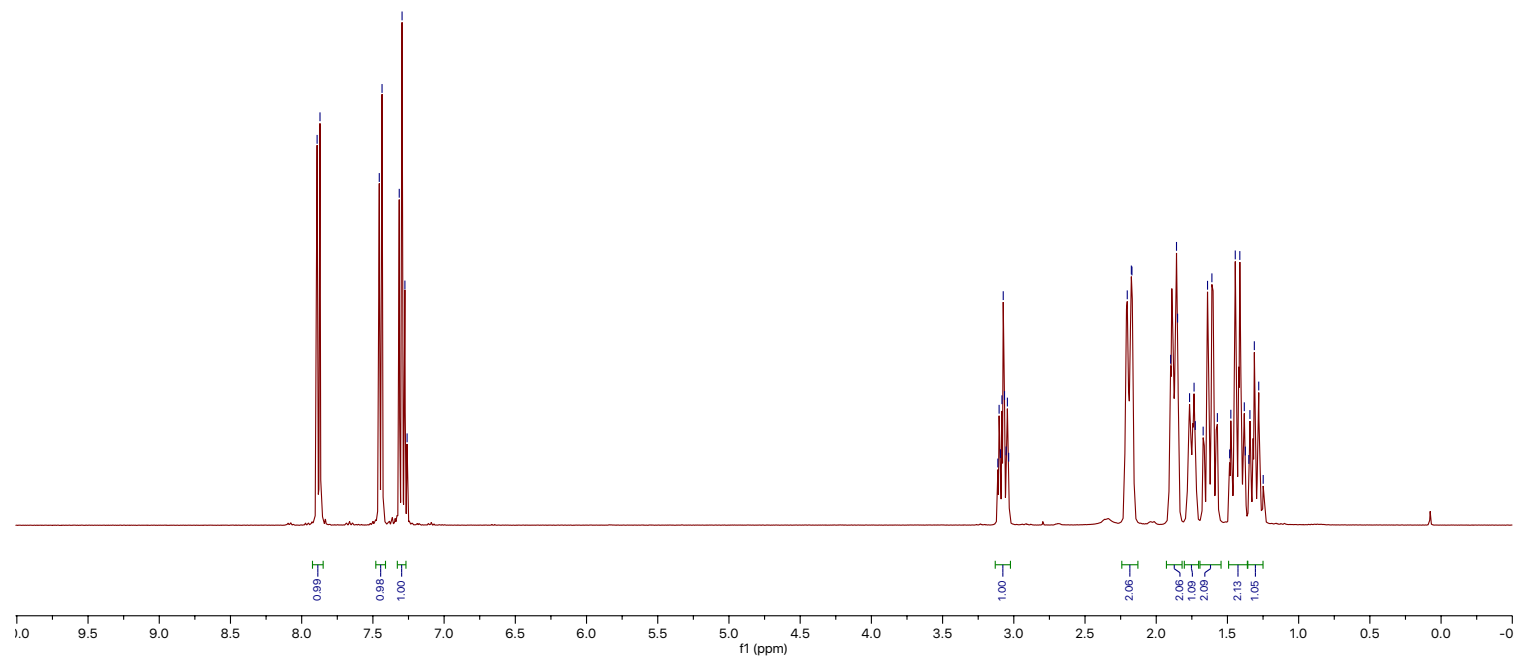

2h-7-48A1

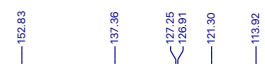

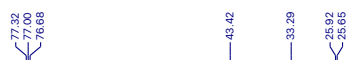

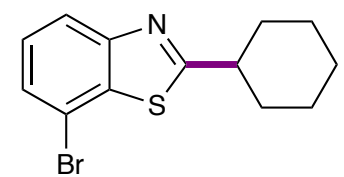

52

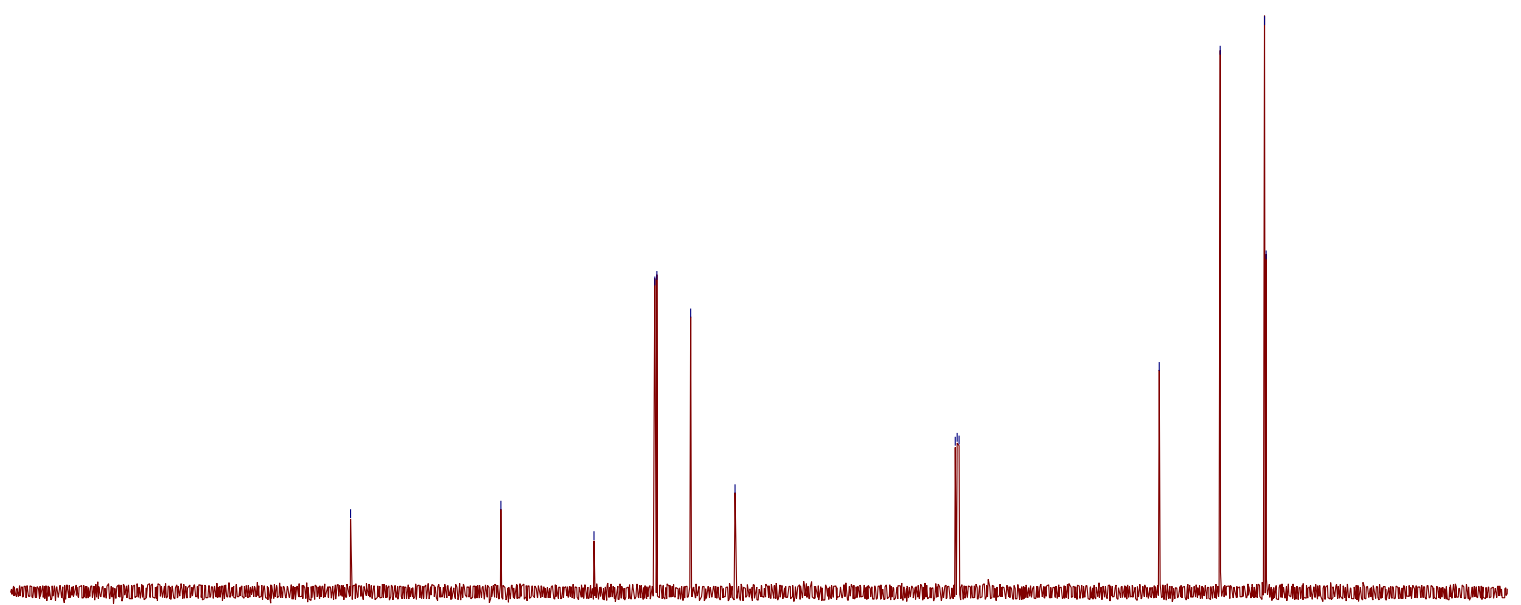

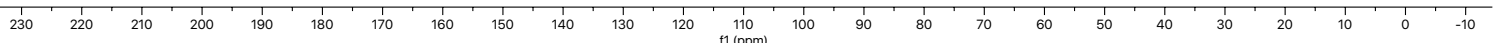




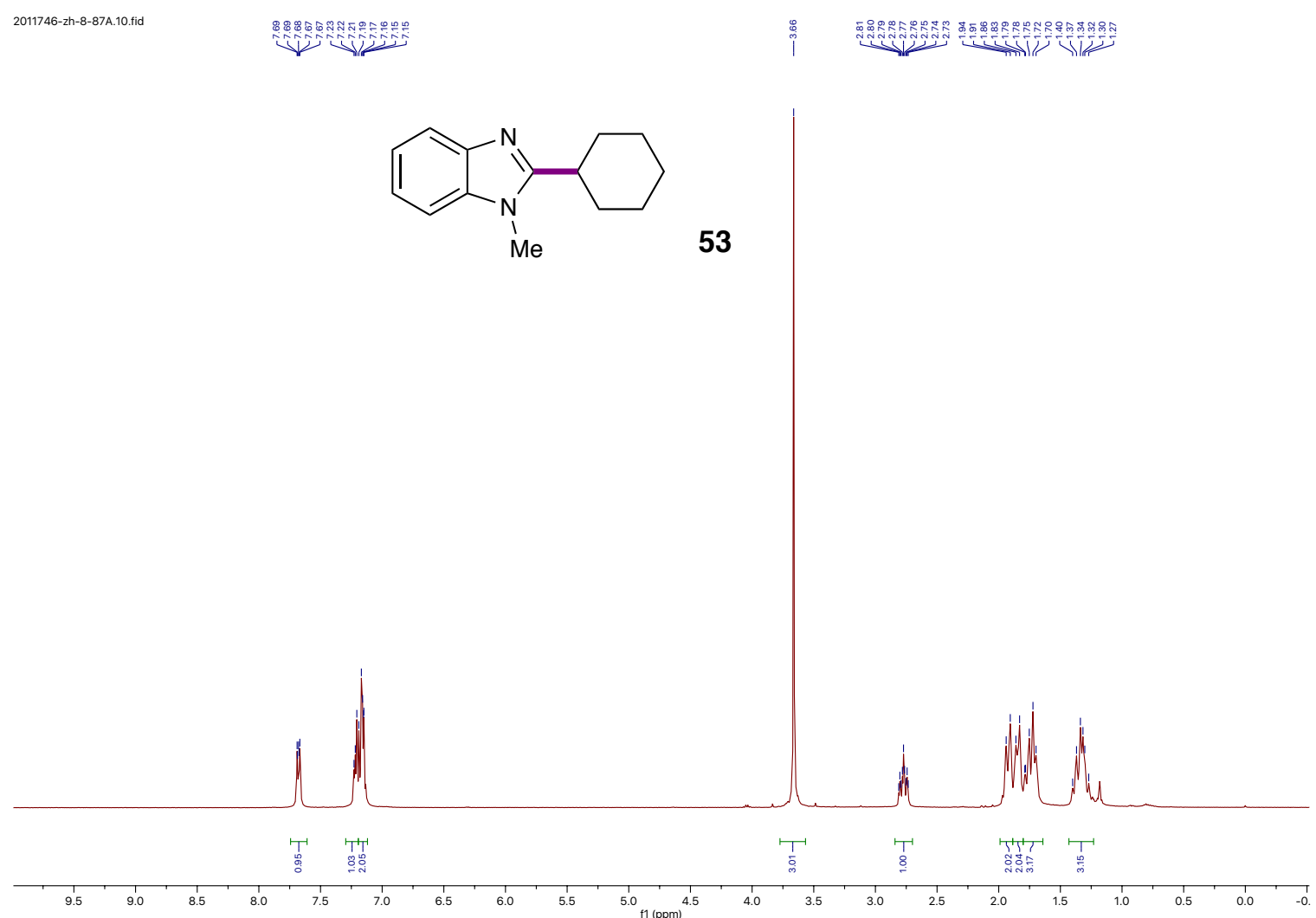

-zh-8-87D

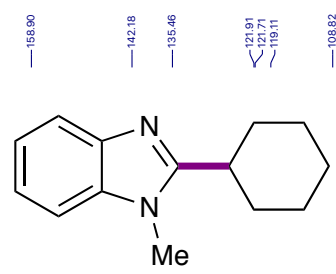

53

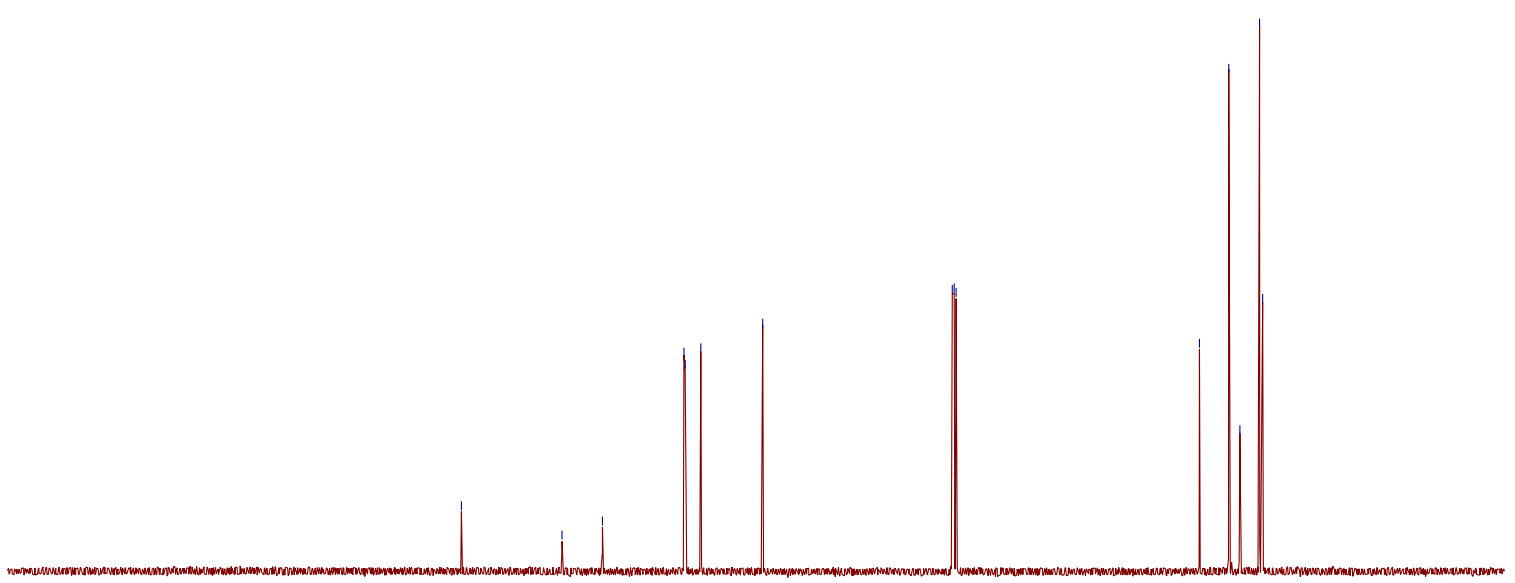

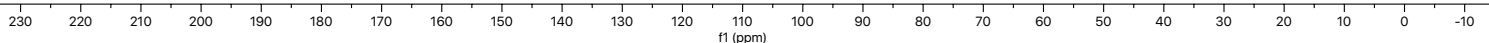



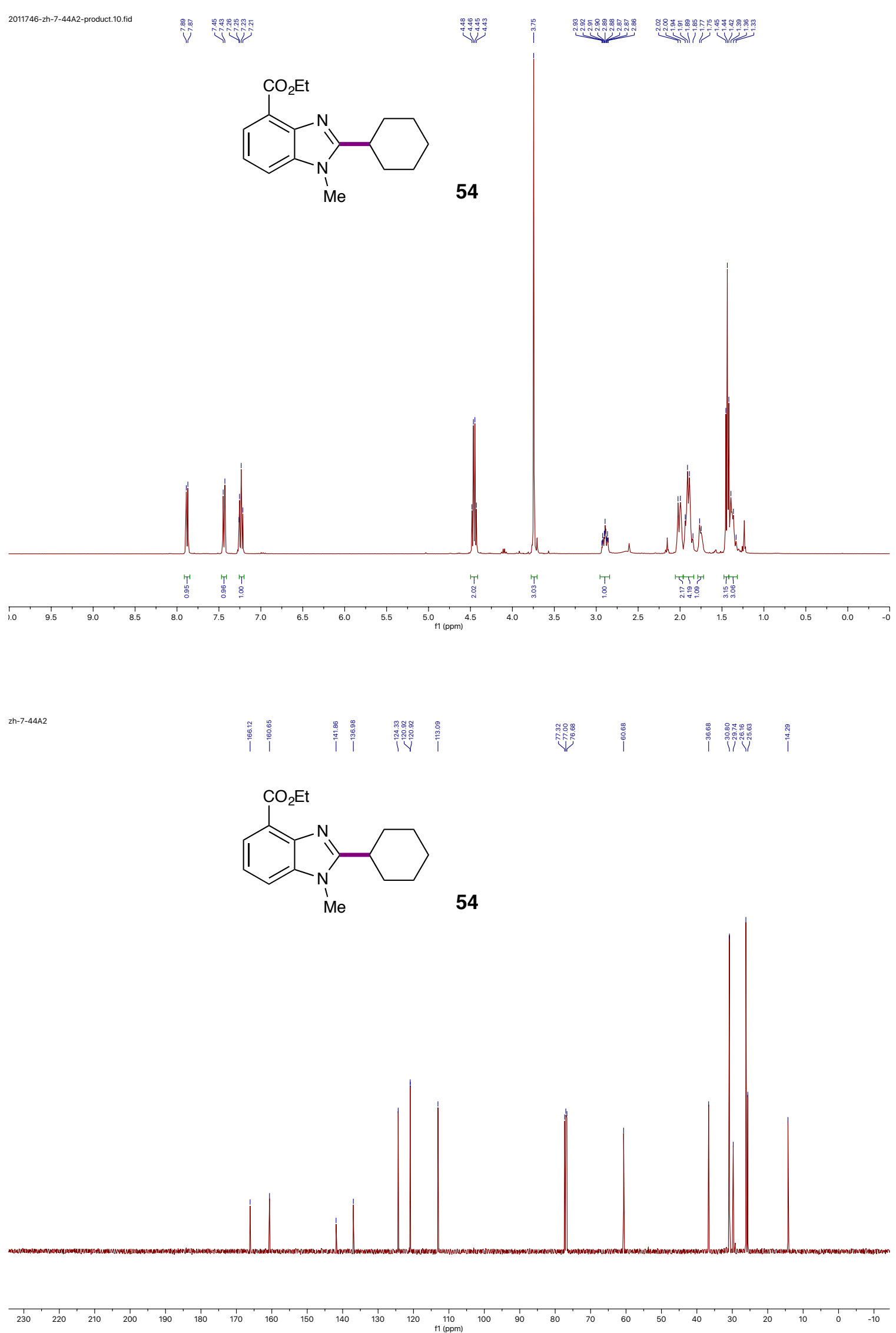


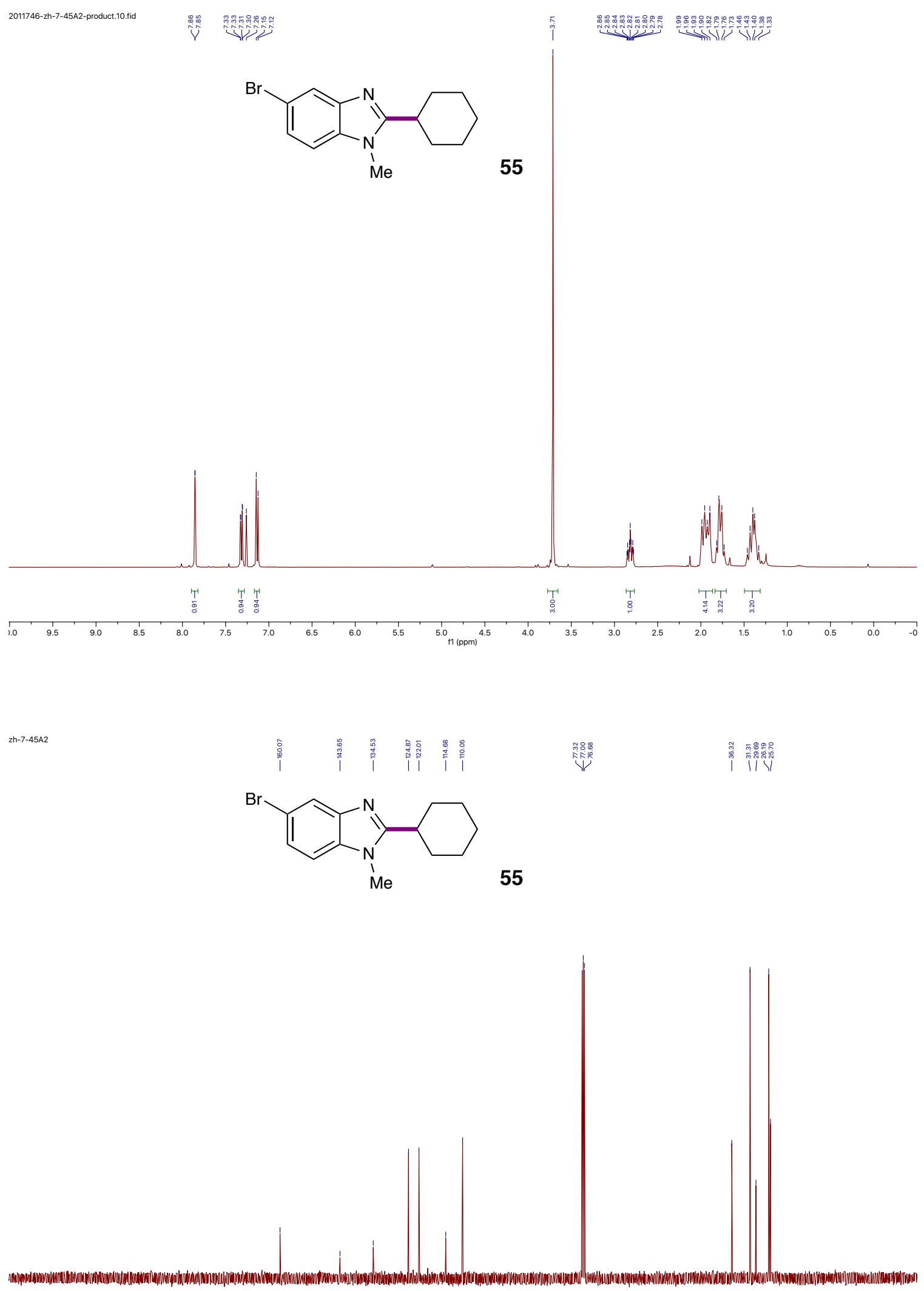

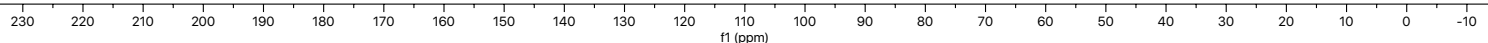




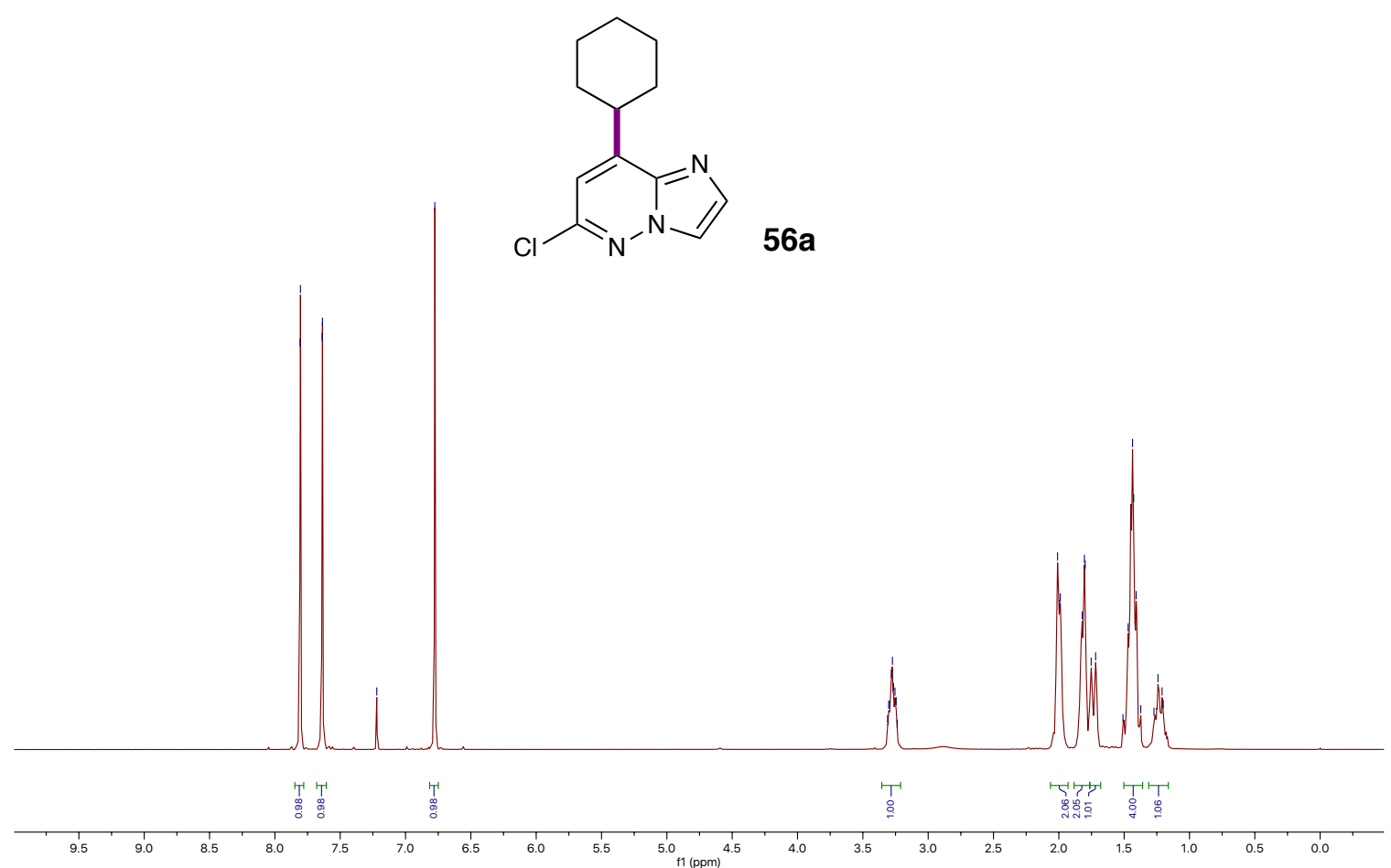

zh-7-39A1
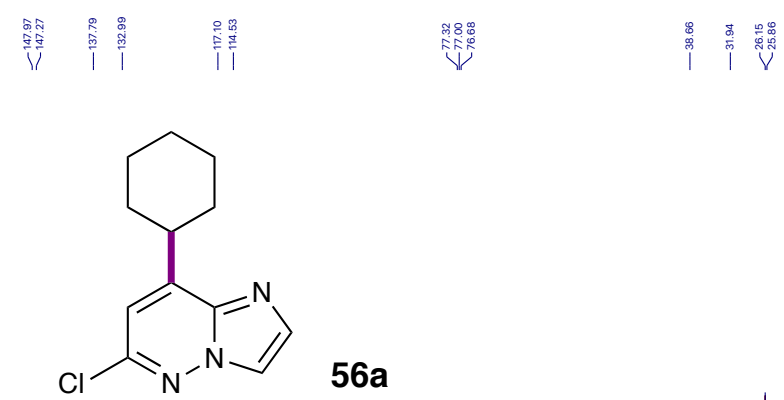

$56 a$

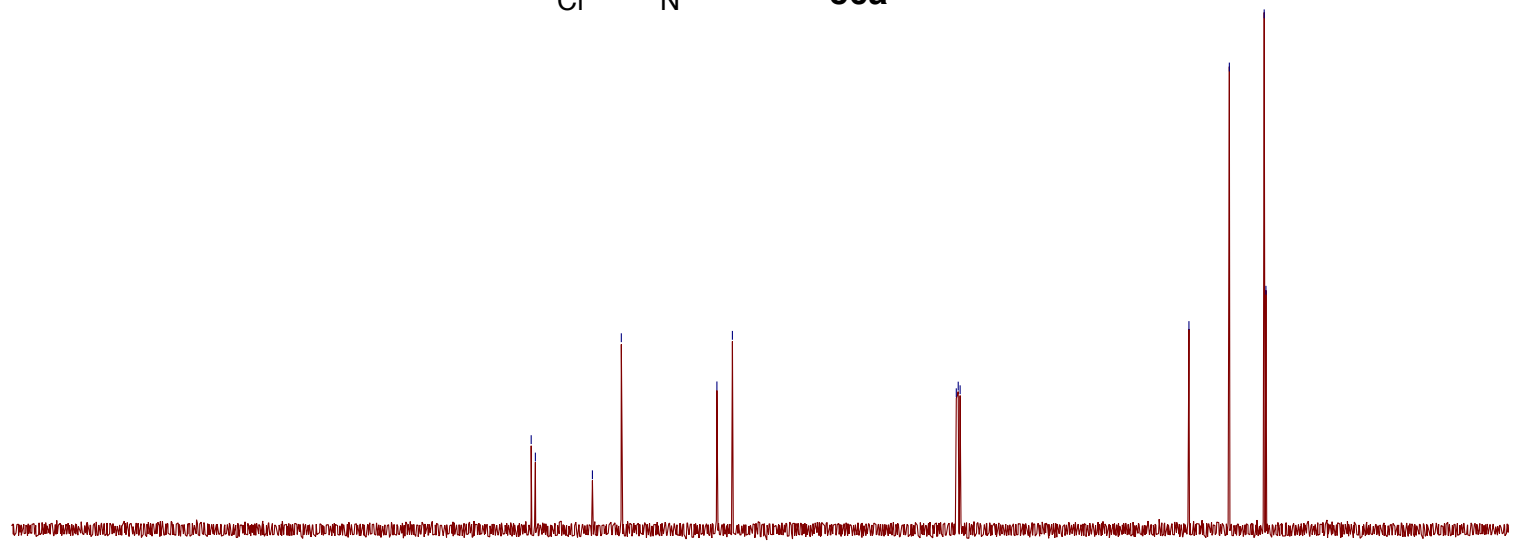

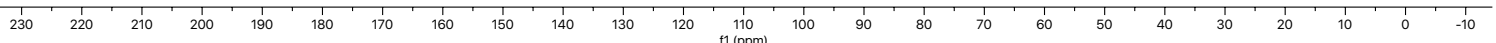



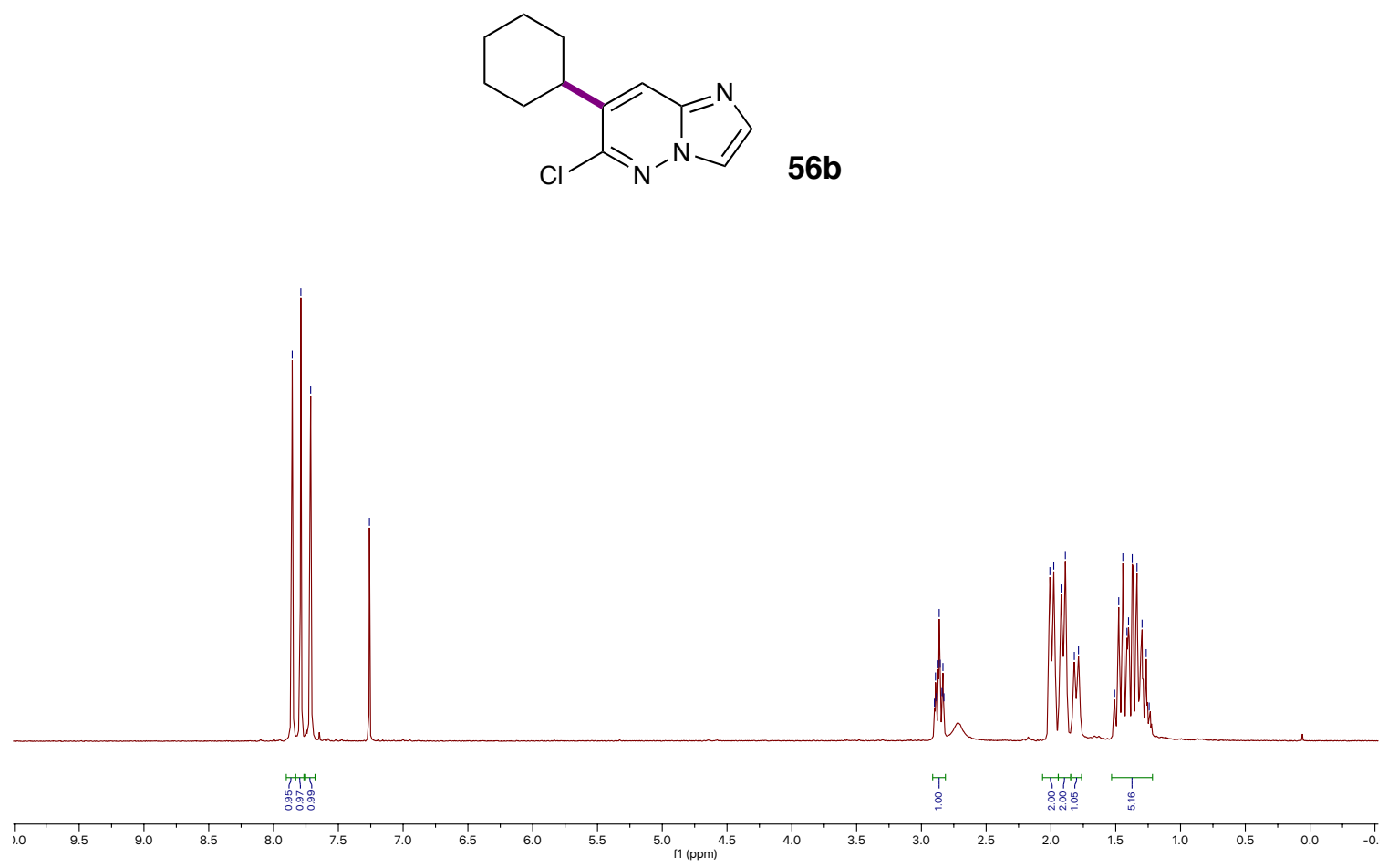

2h-7-39A2

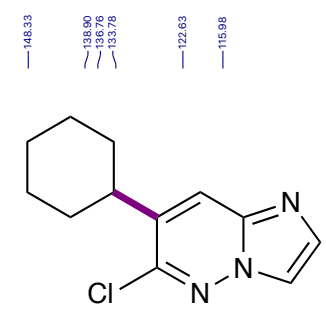

的

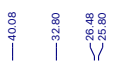

$56 \mathrm{~b}$

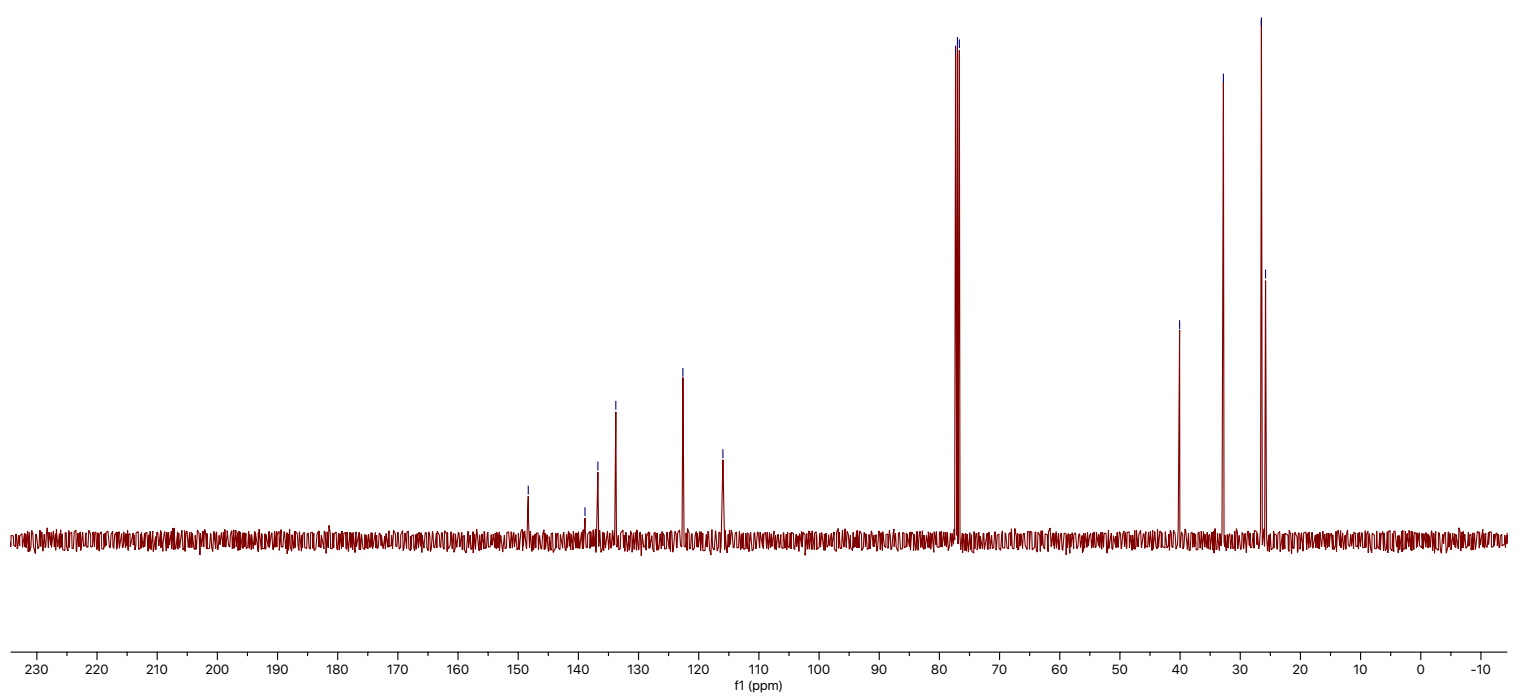




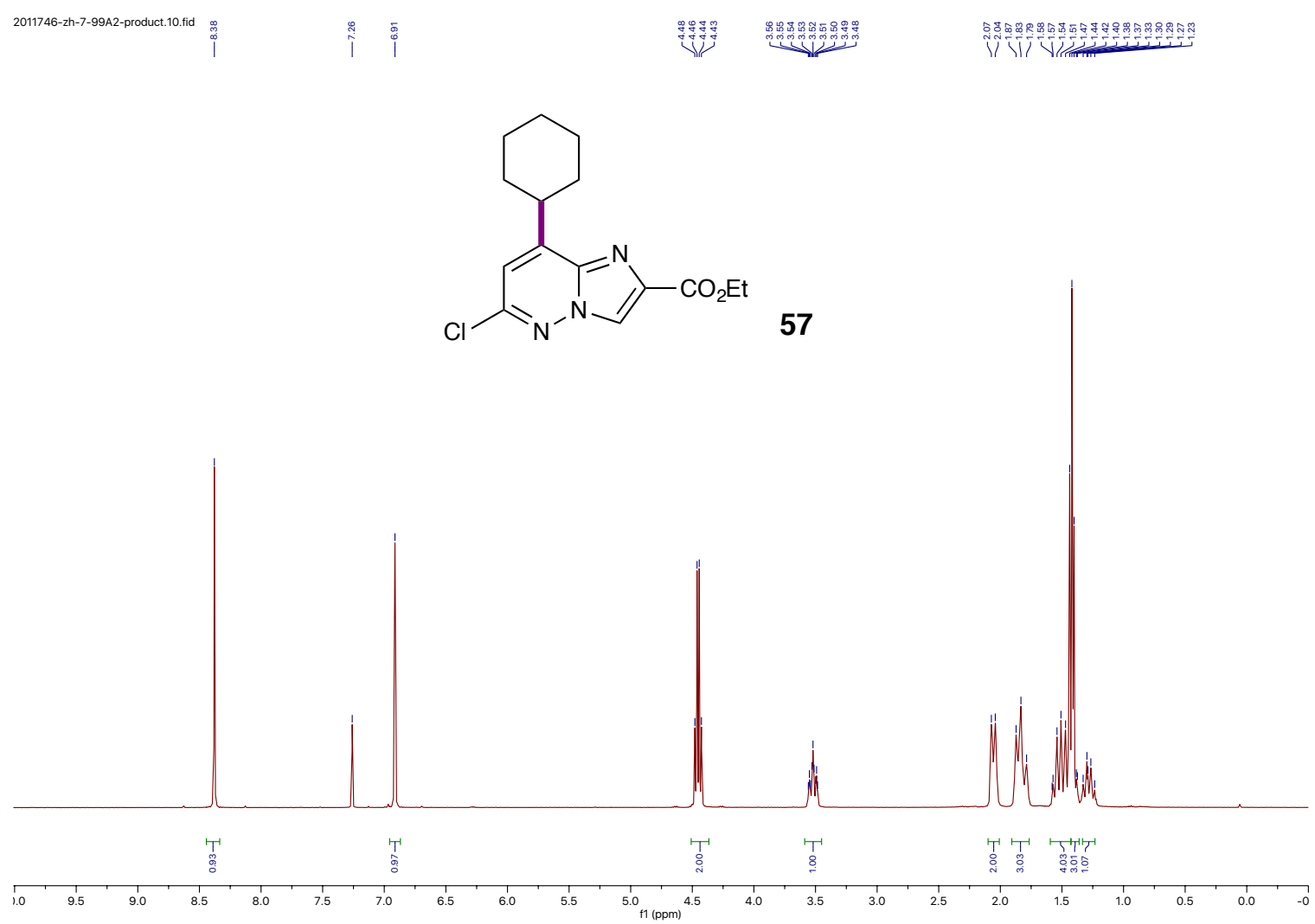

zh7-99A2
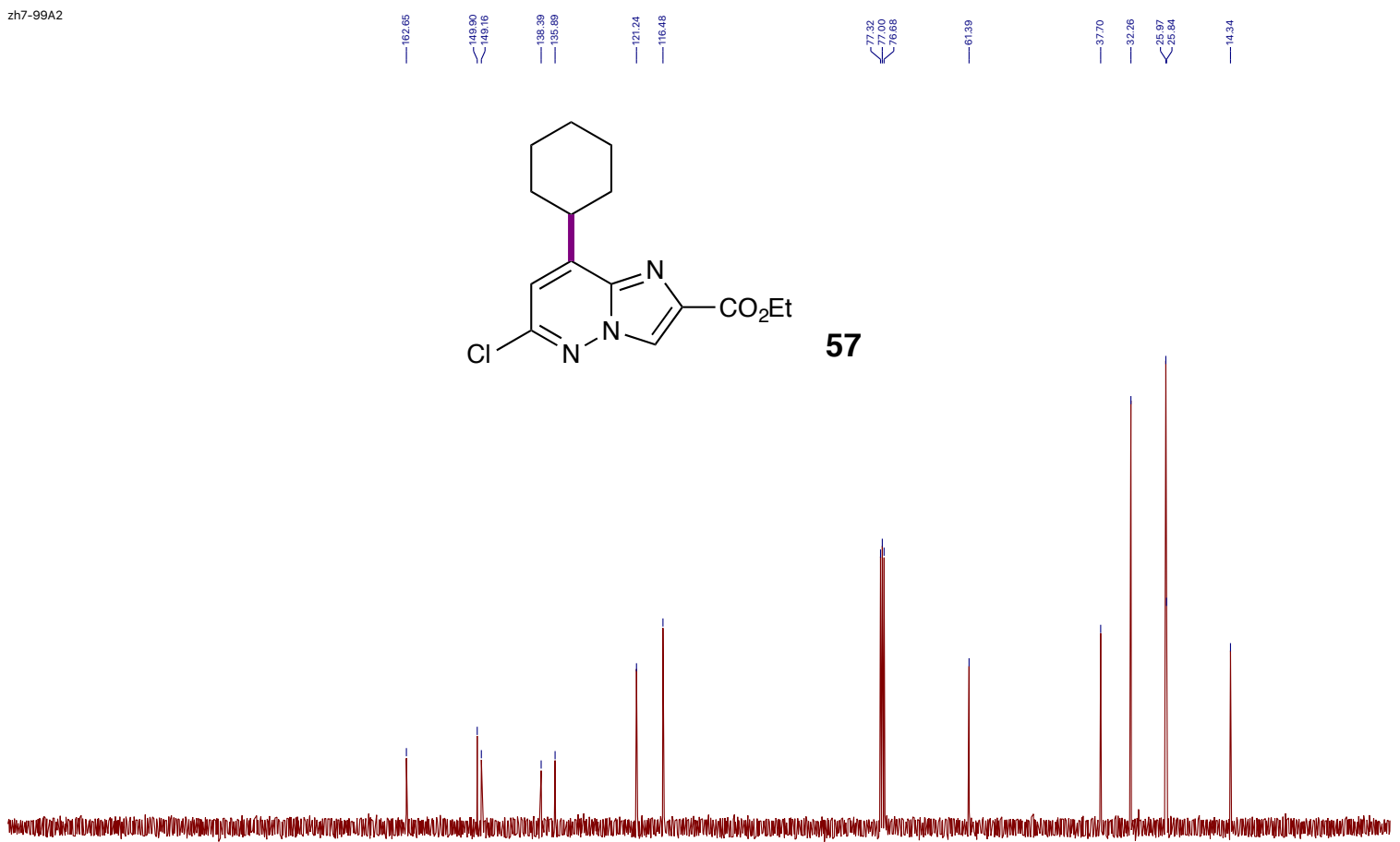

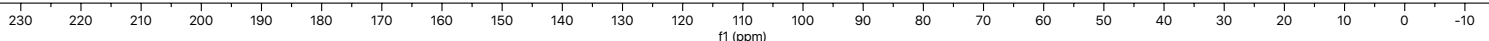




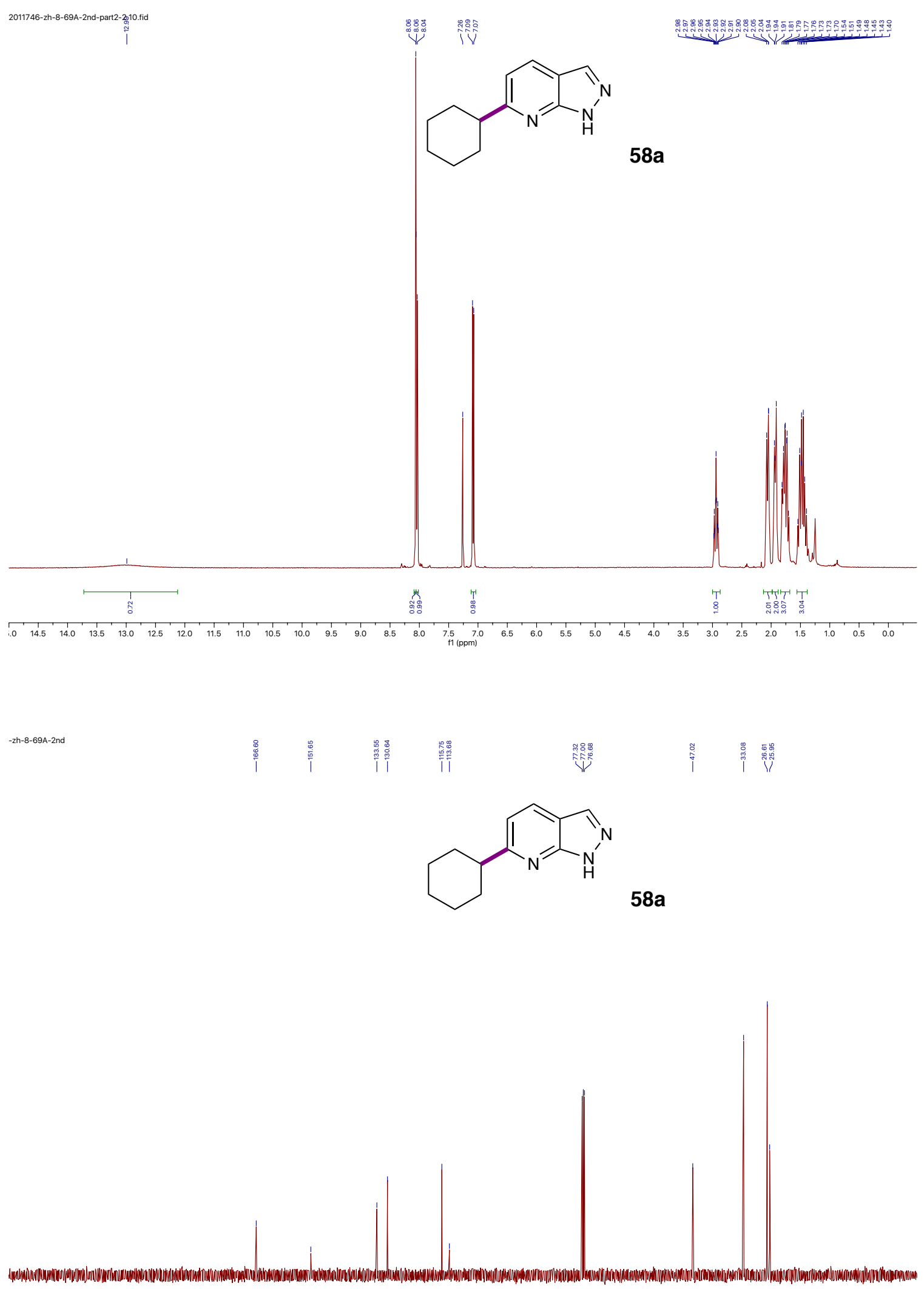

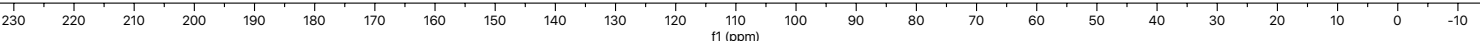




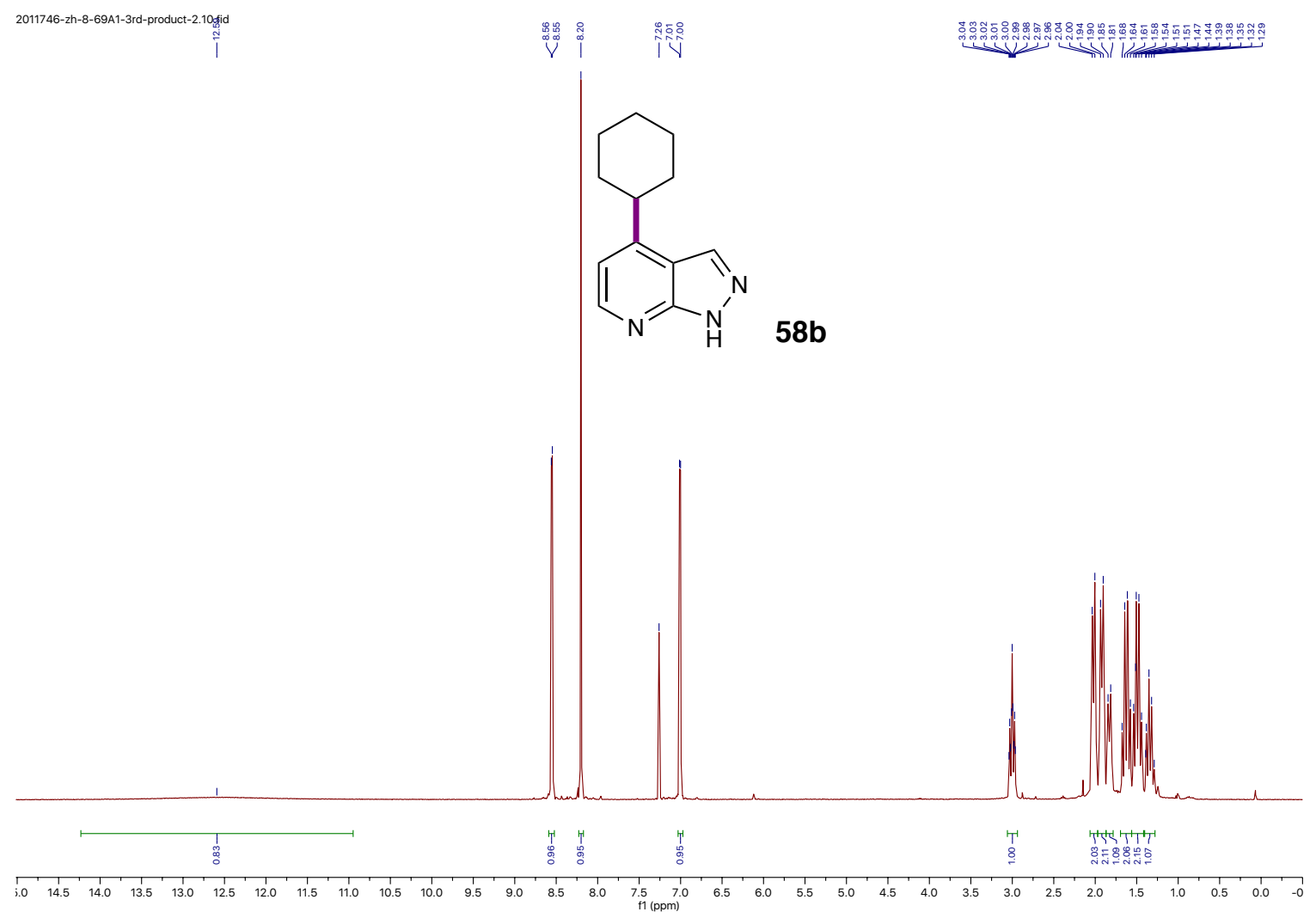

zh8-69A1-3rd
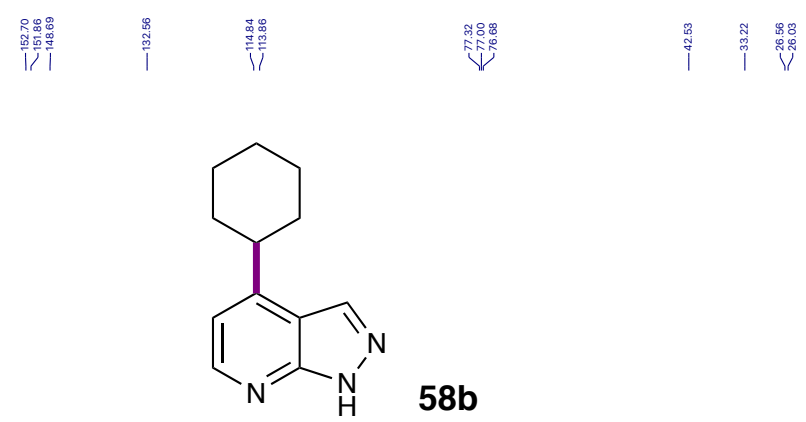

$58 b$

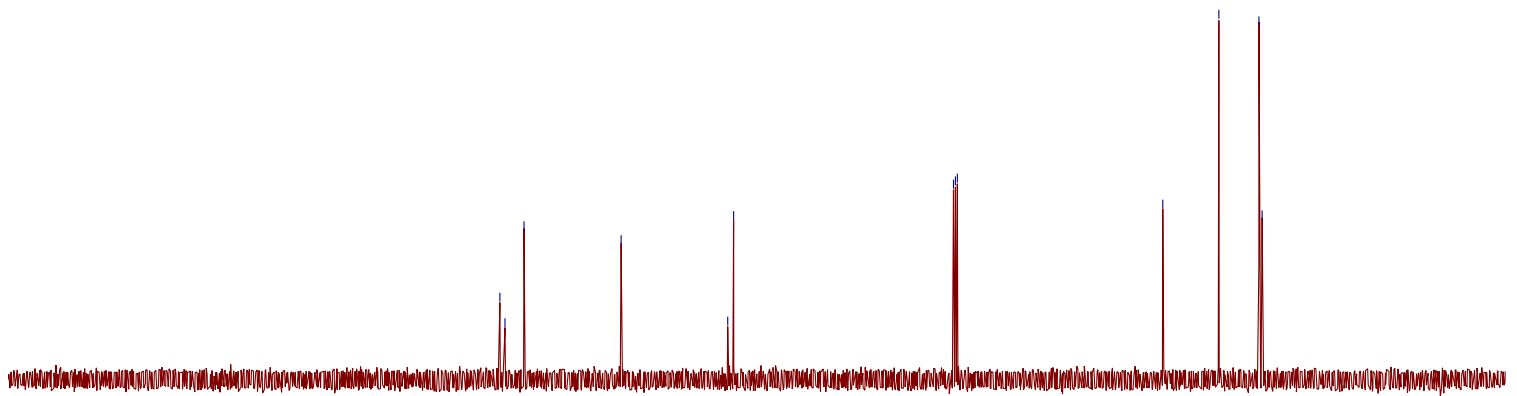

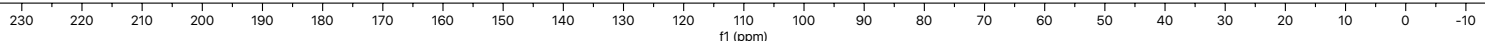




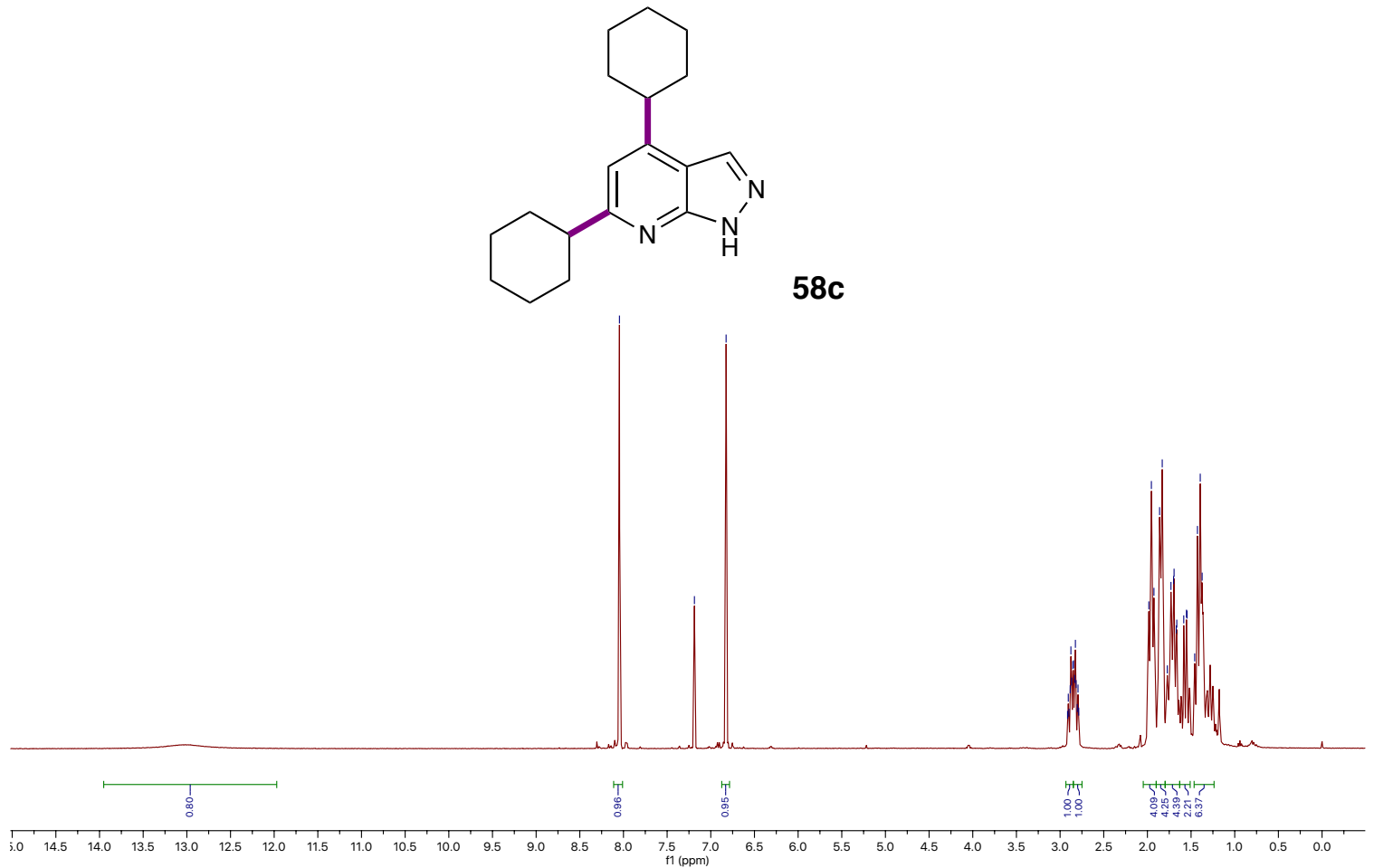

zh8-69A1-1st

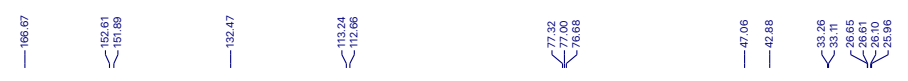<smiles>c1nc2[nH]ncc2cc1C1CCCCC1</smiles>

$58 c$

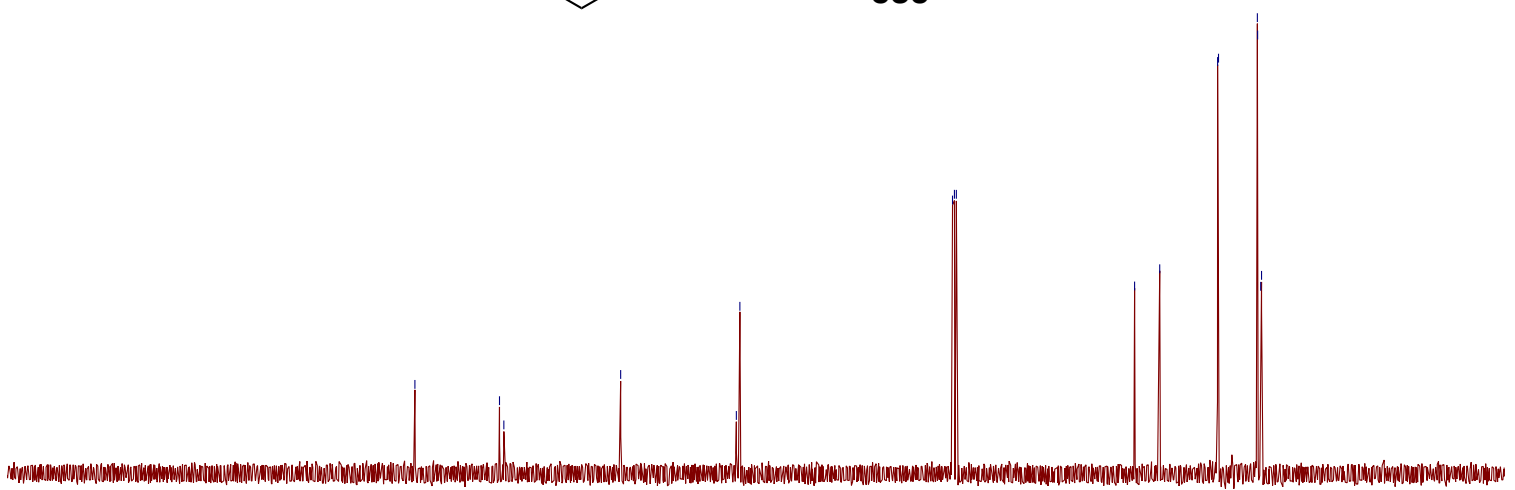

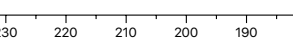

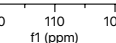



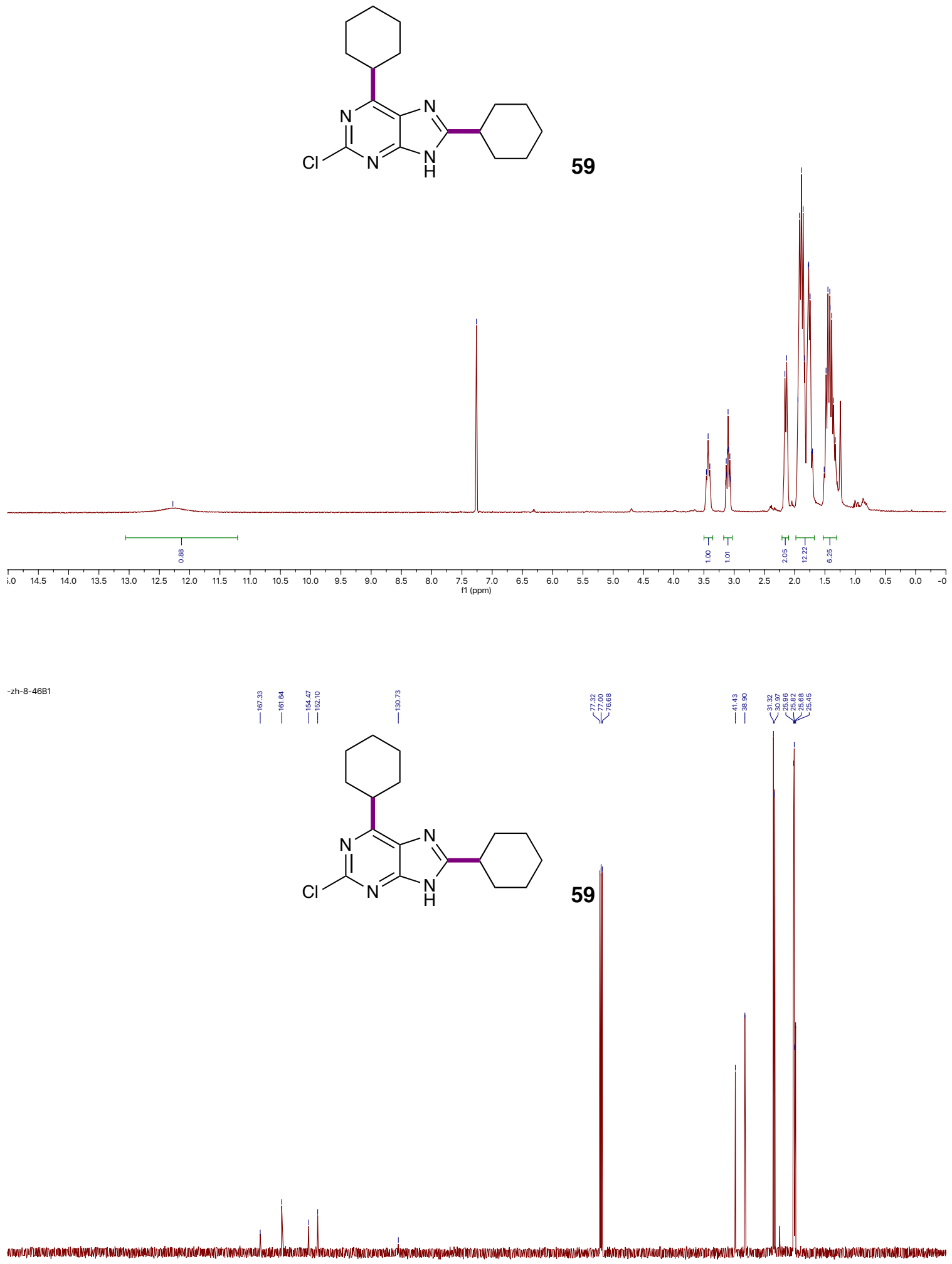


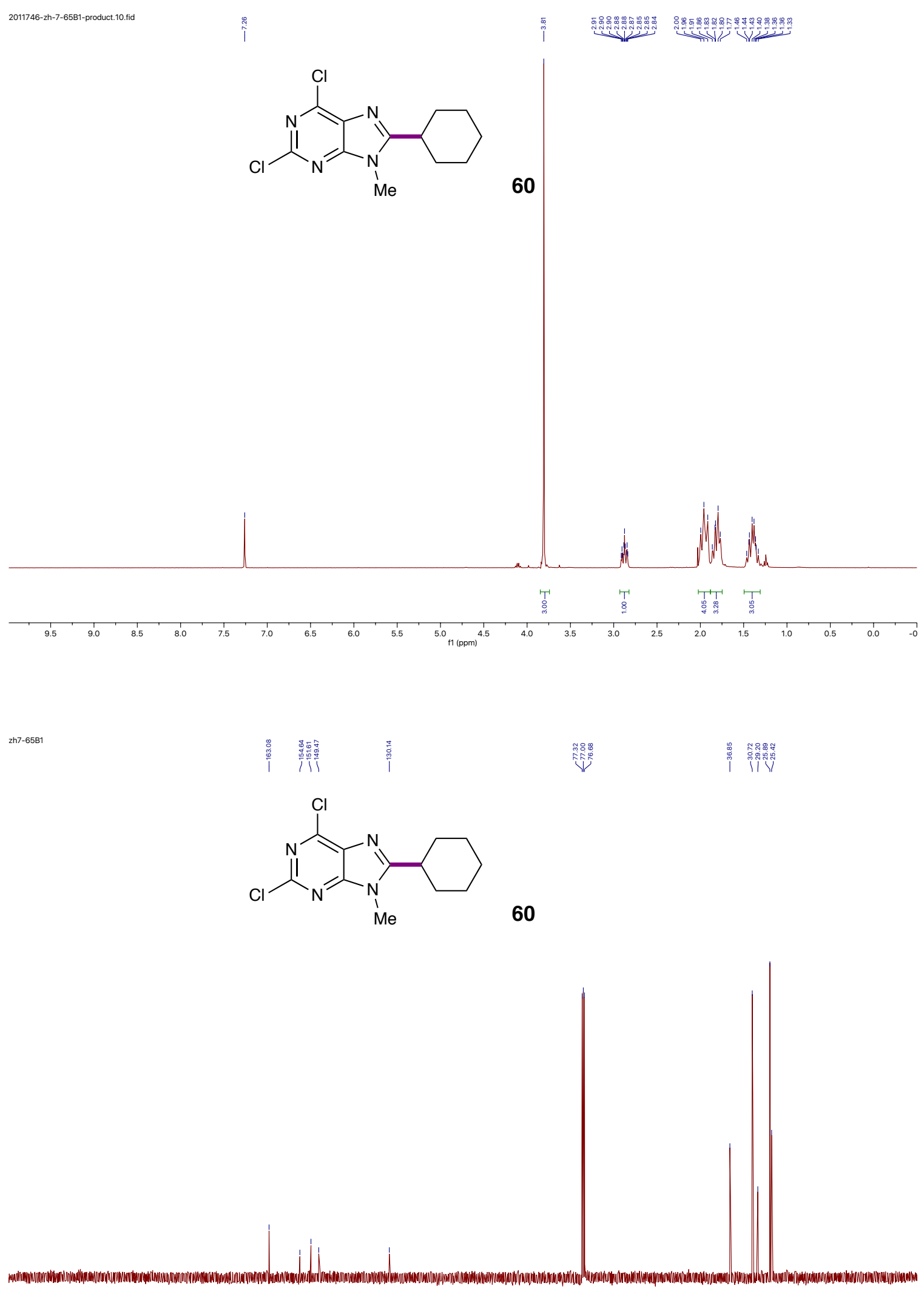

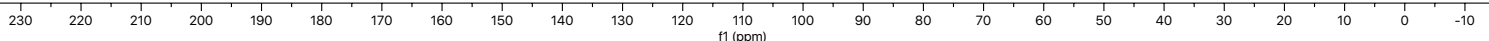




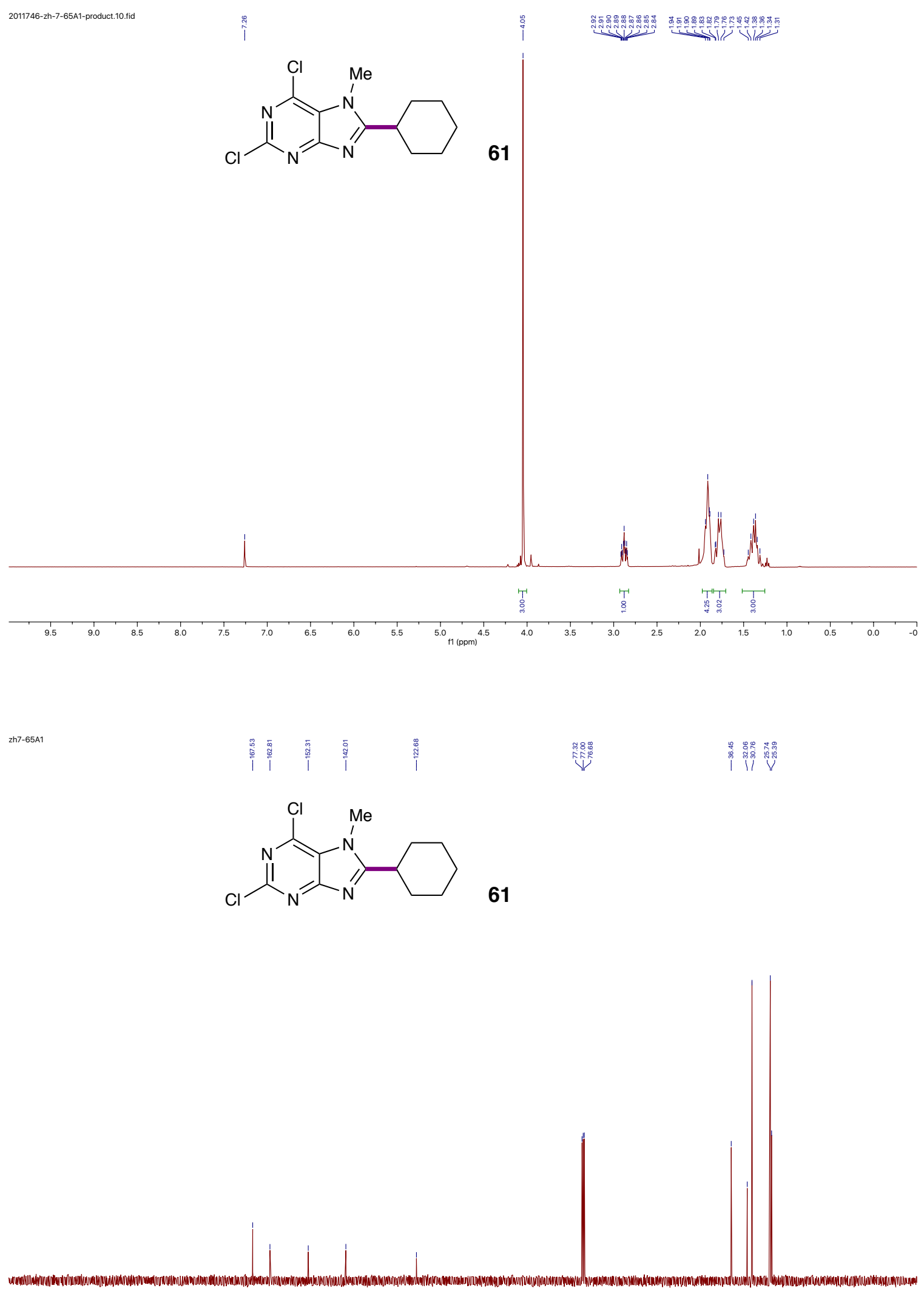

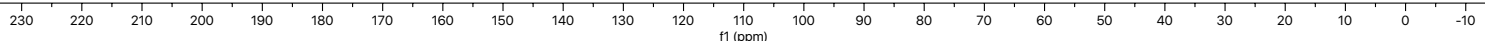



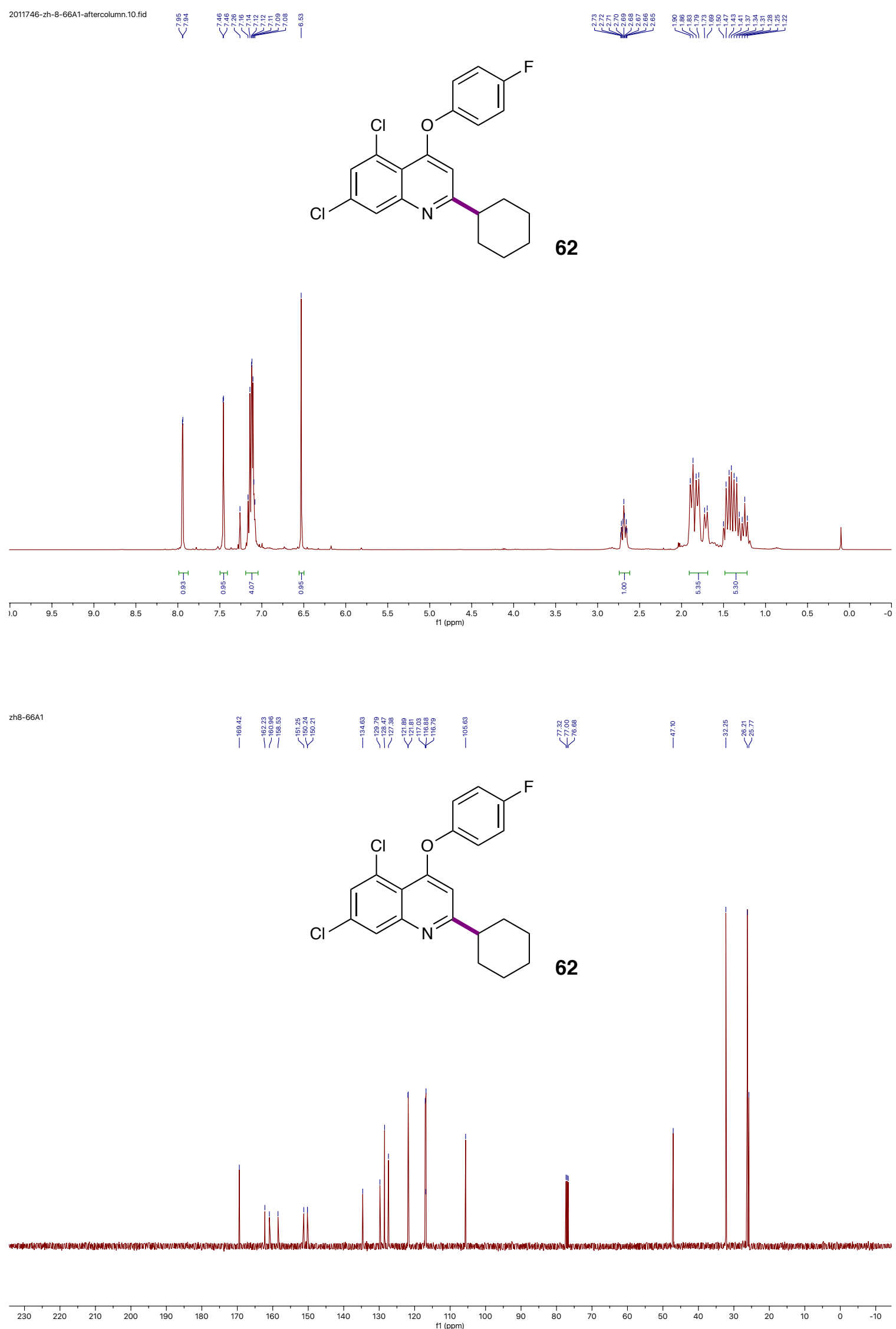

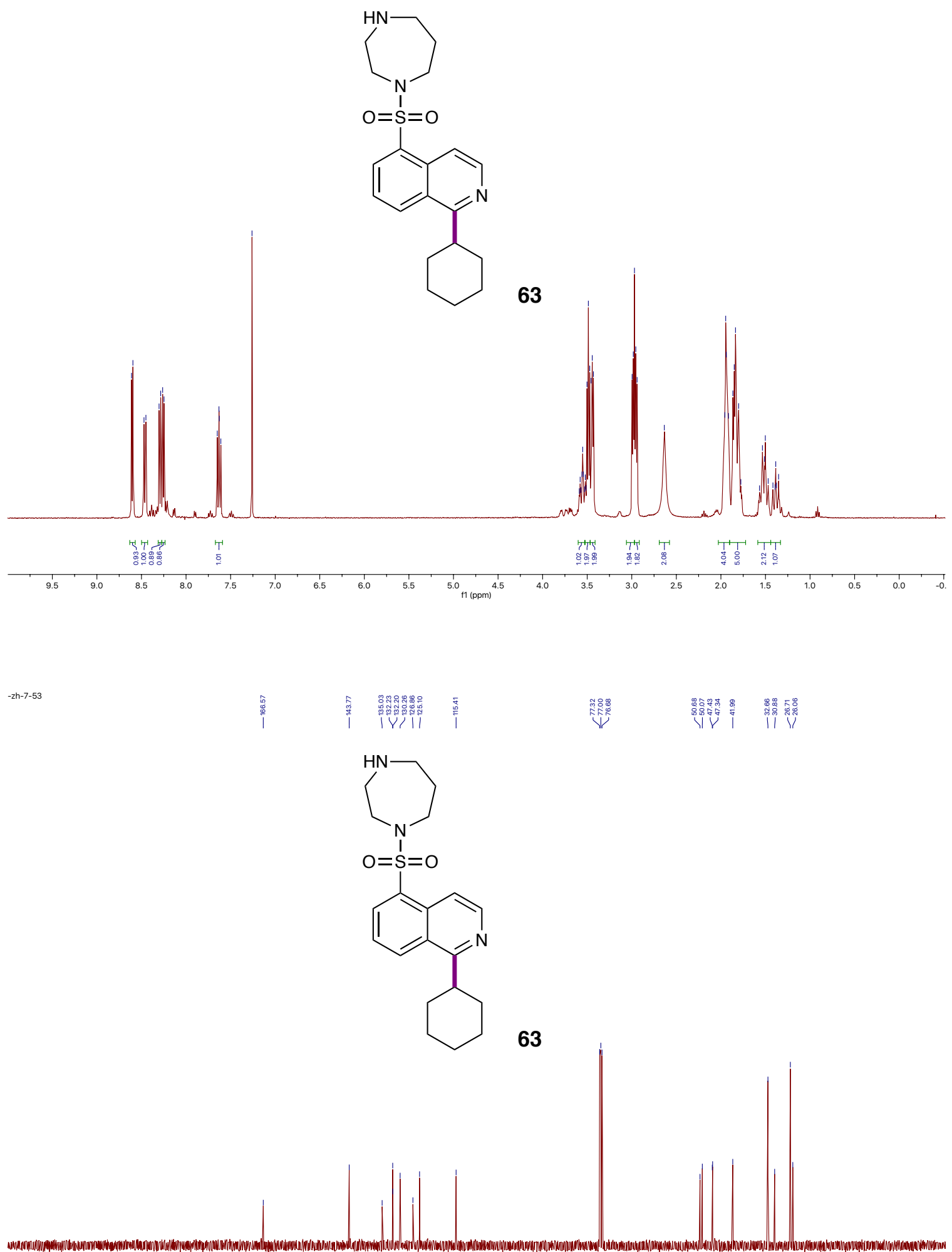

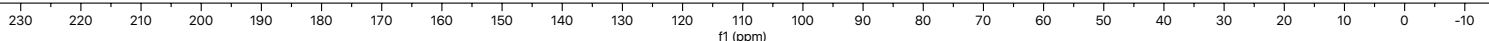




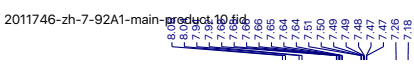

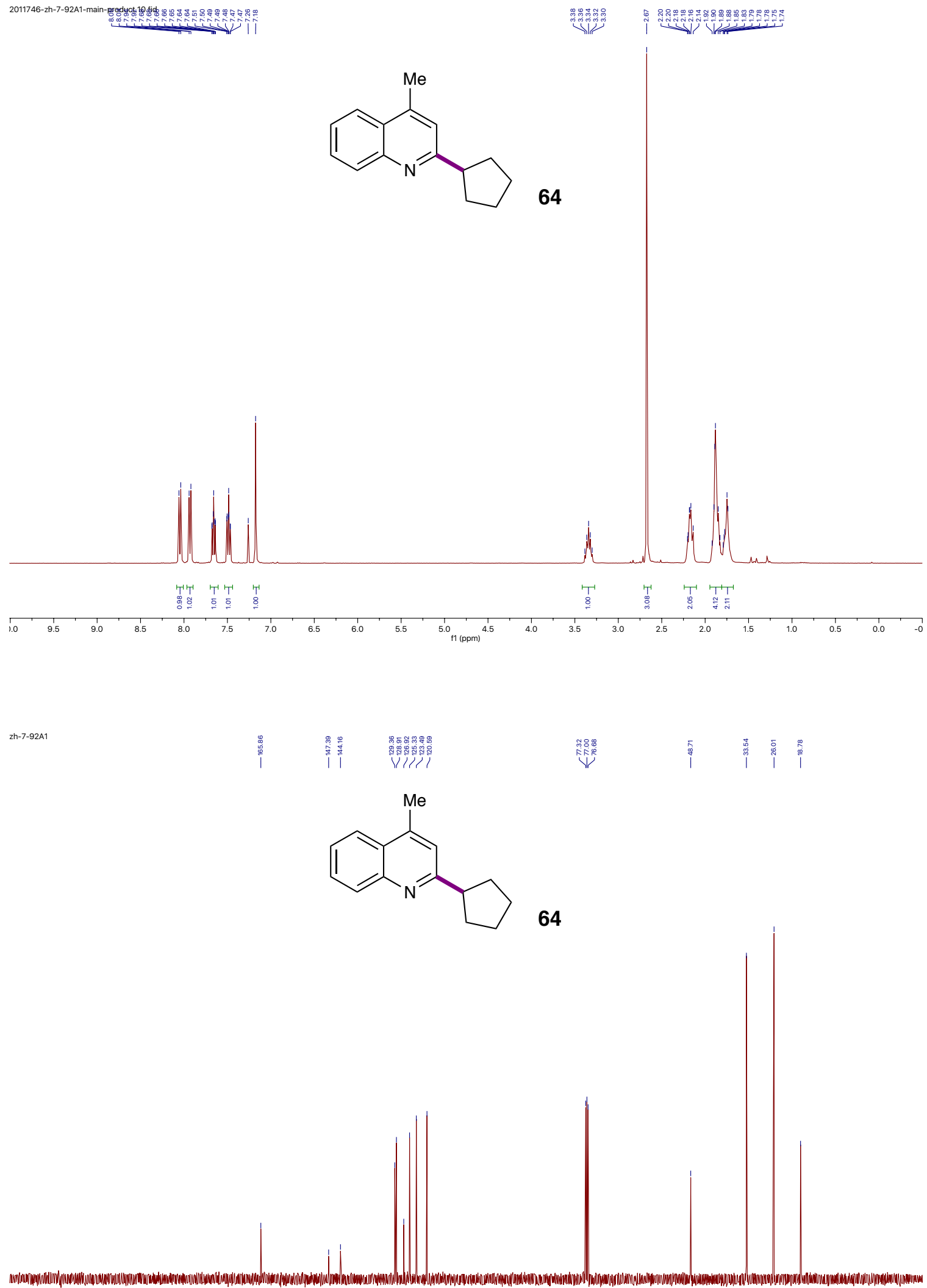

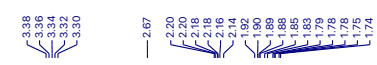

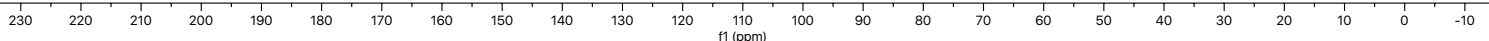



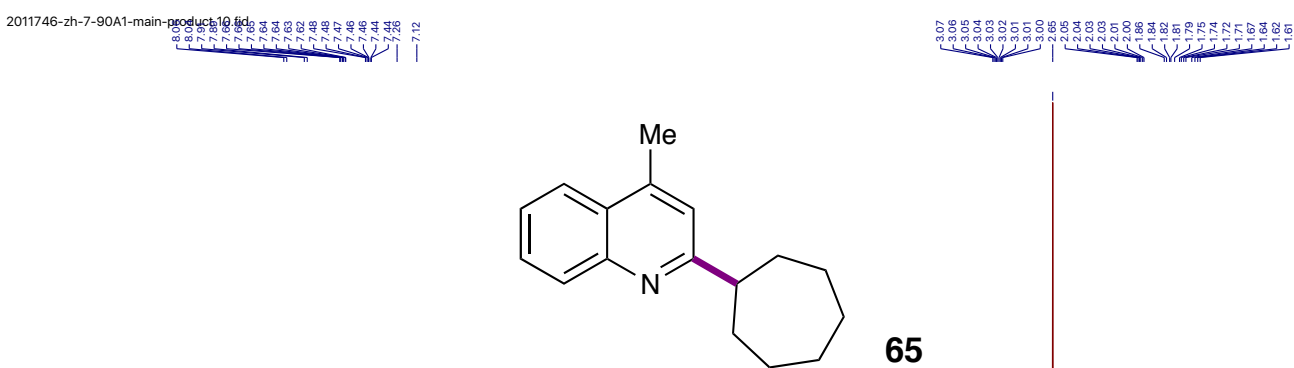

65

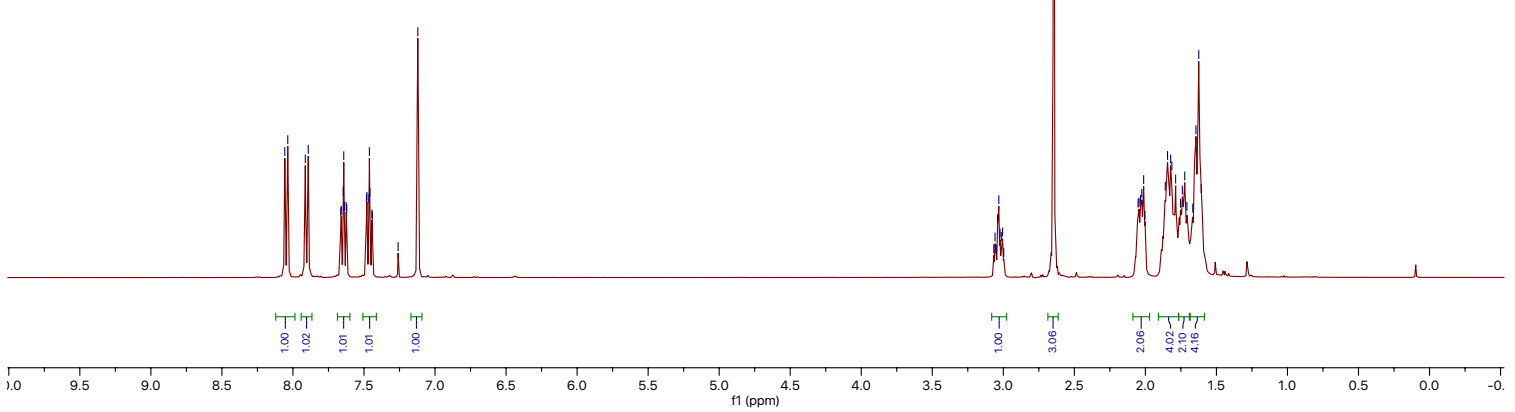

zh-7-90A1

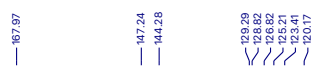

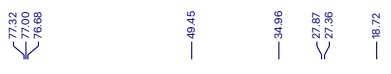

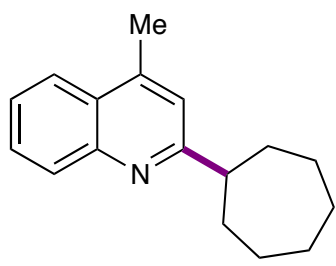

65

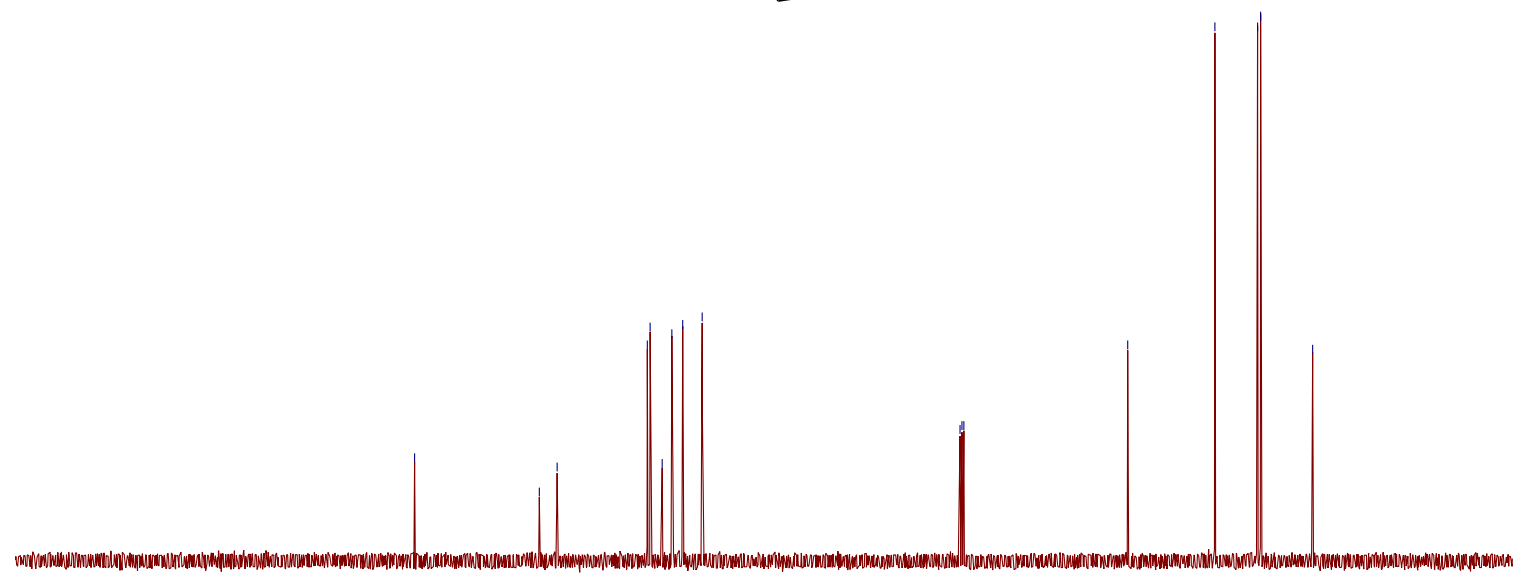

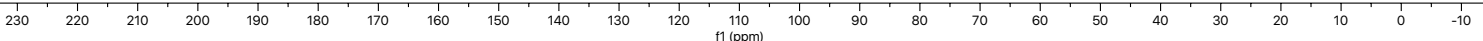




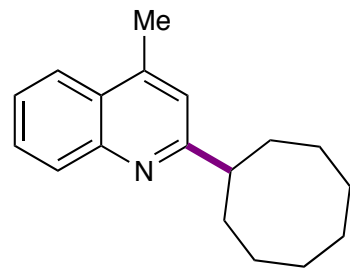

66

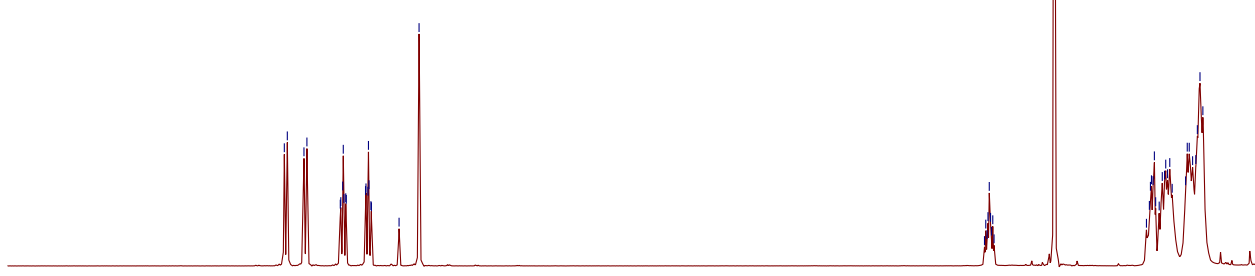

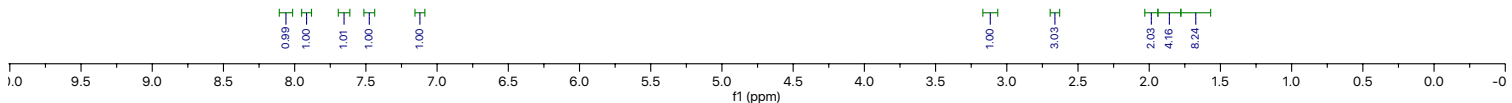

Zh-7-91A1

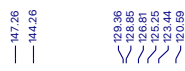

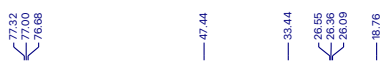

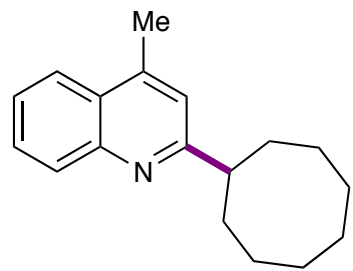

66

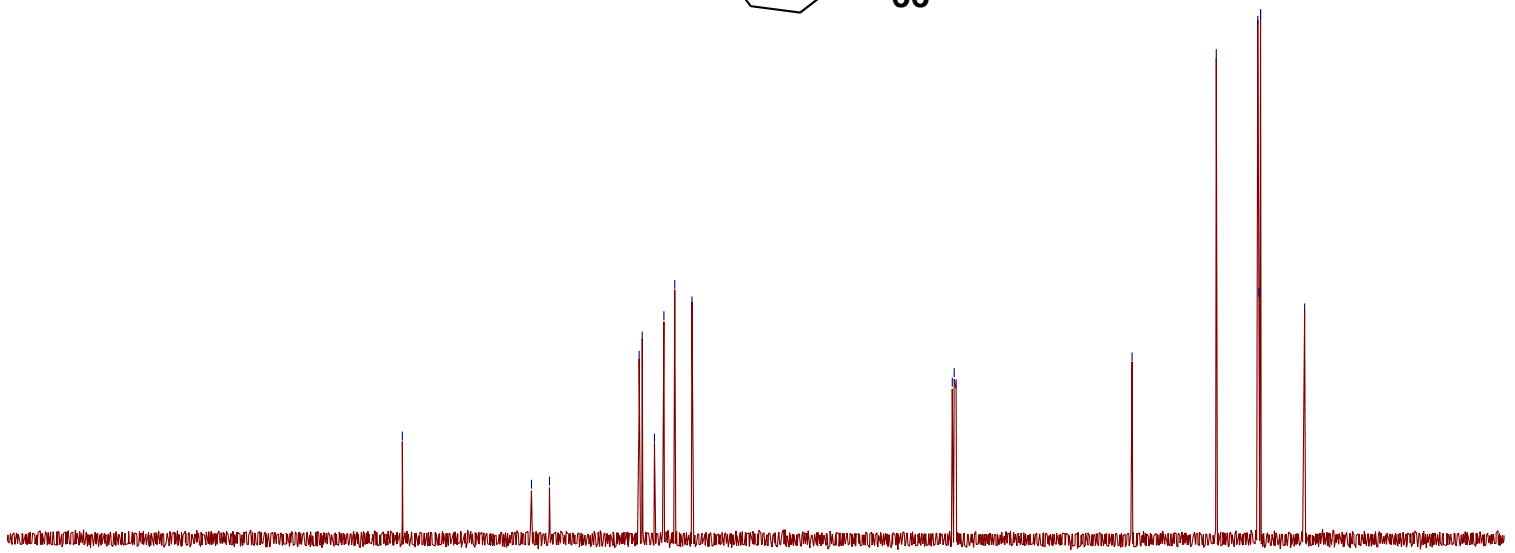

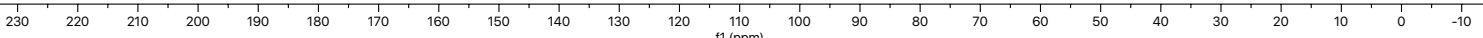




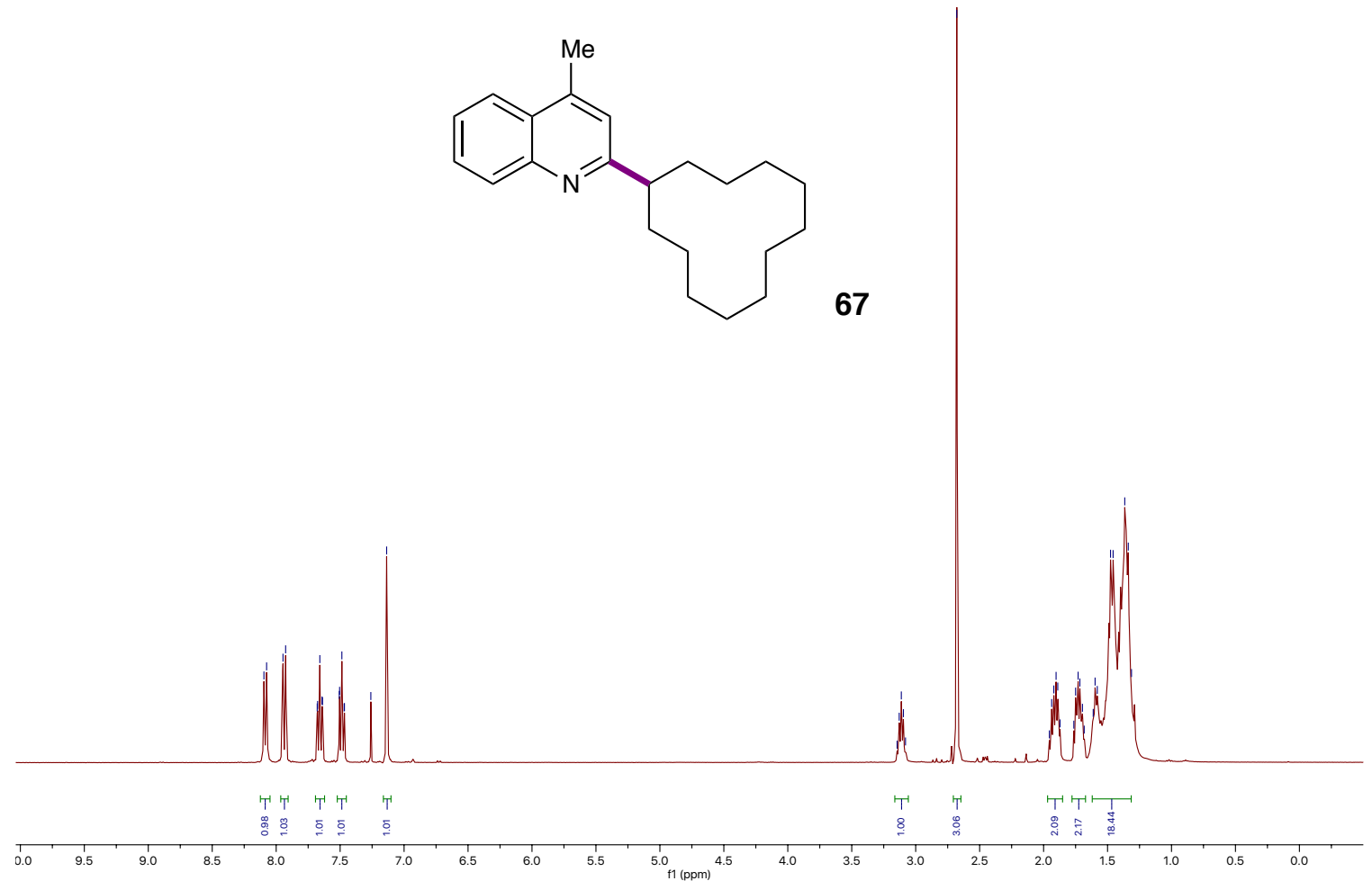

zh-7-95A1
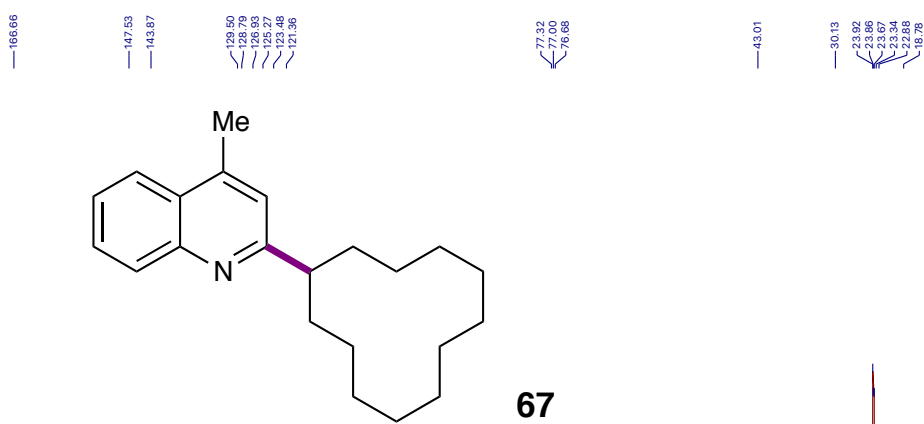

67

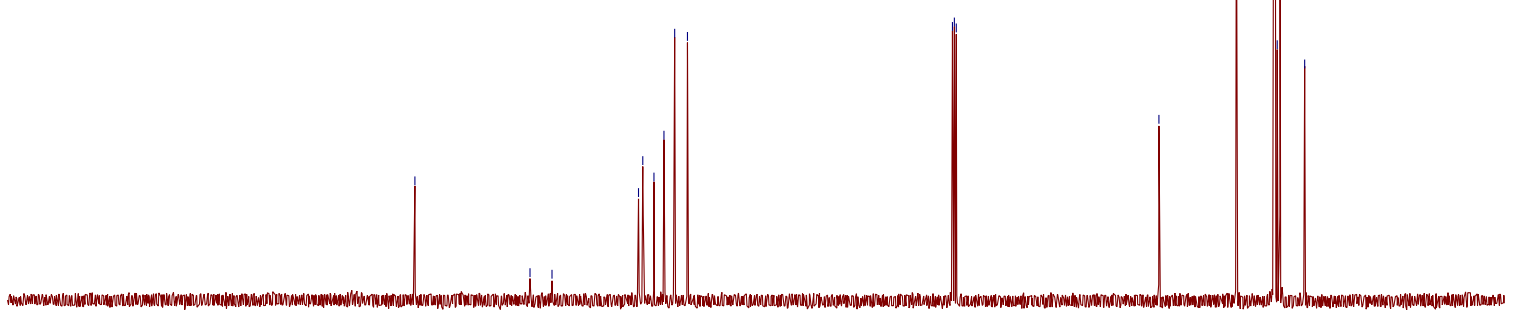

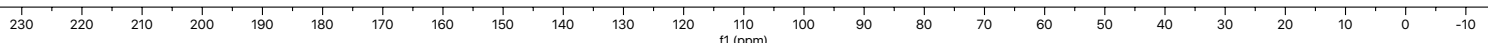




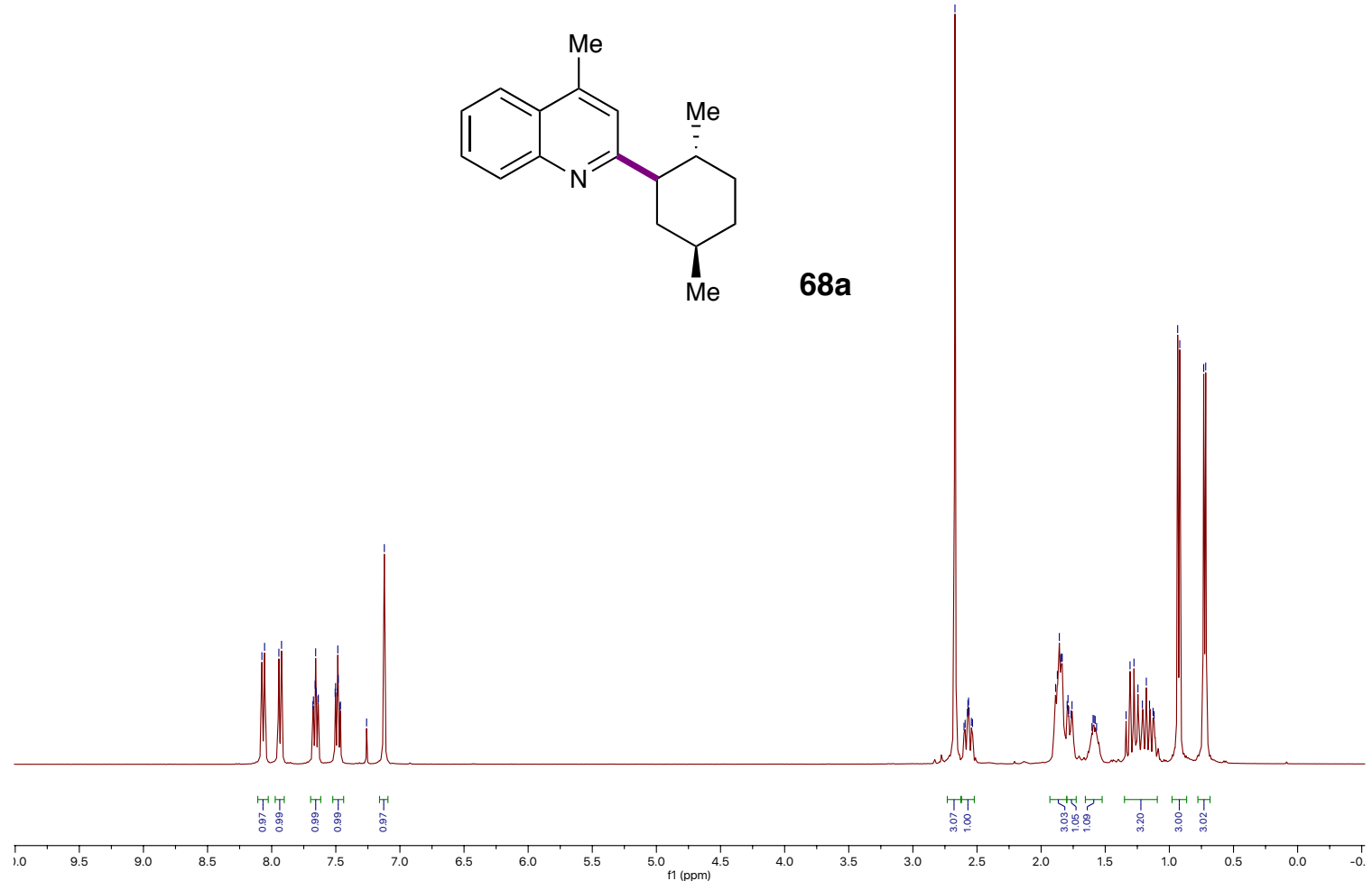

zh-9-22B-2nd

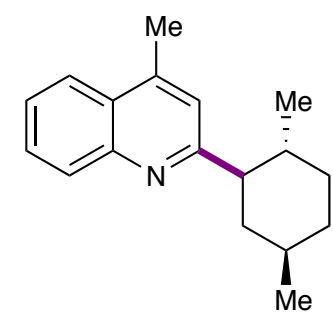

$68 a$

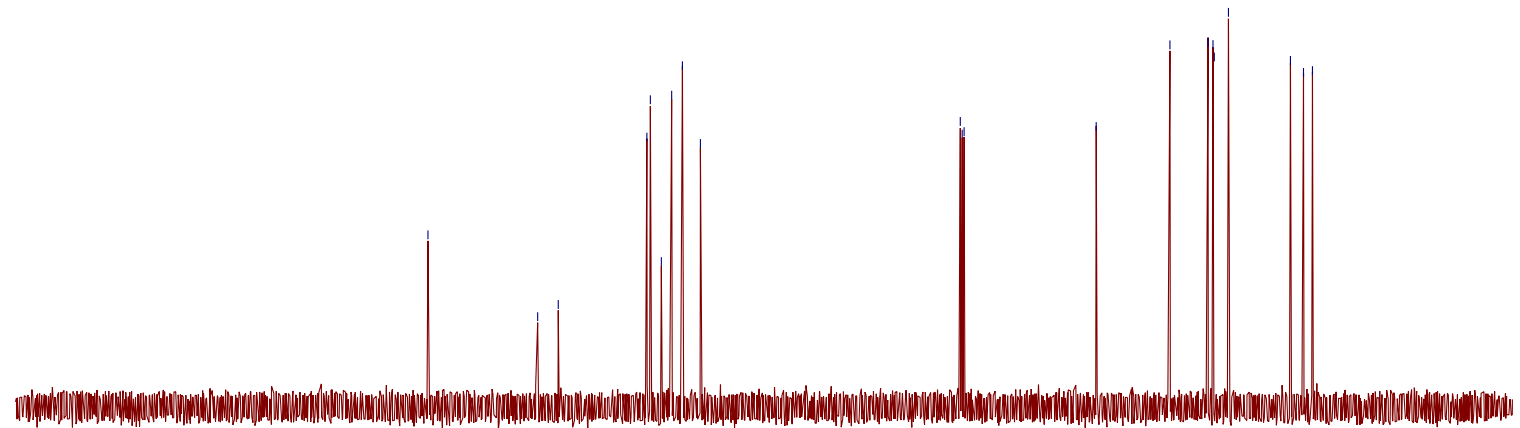

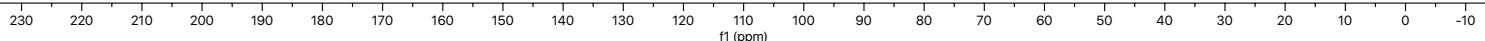


2011746-zh-9-22B-1st-product.10.fid
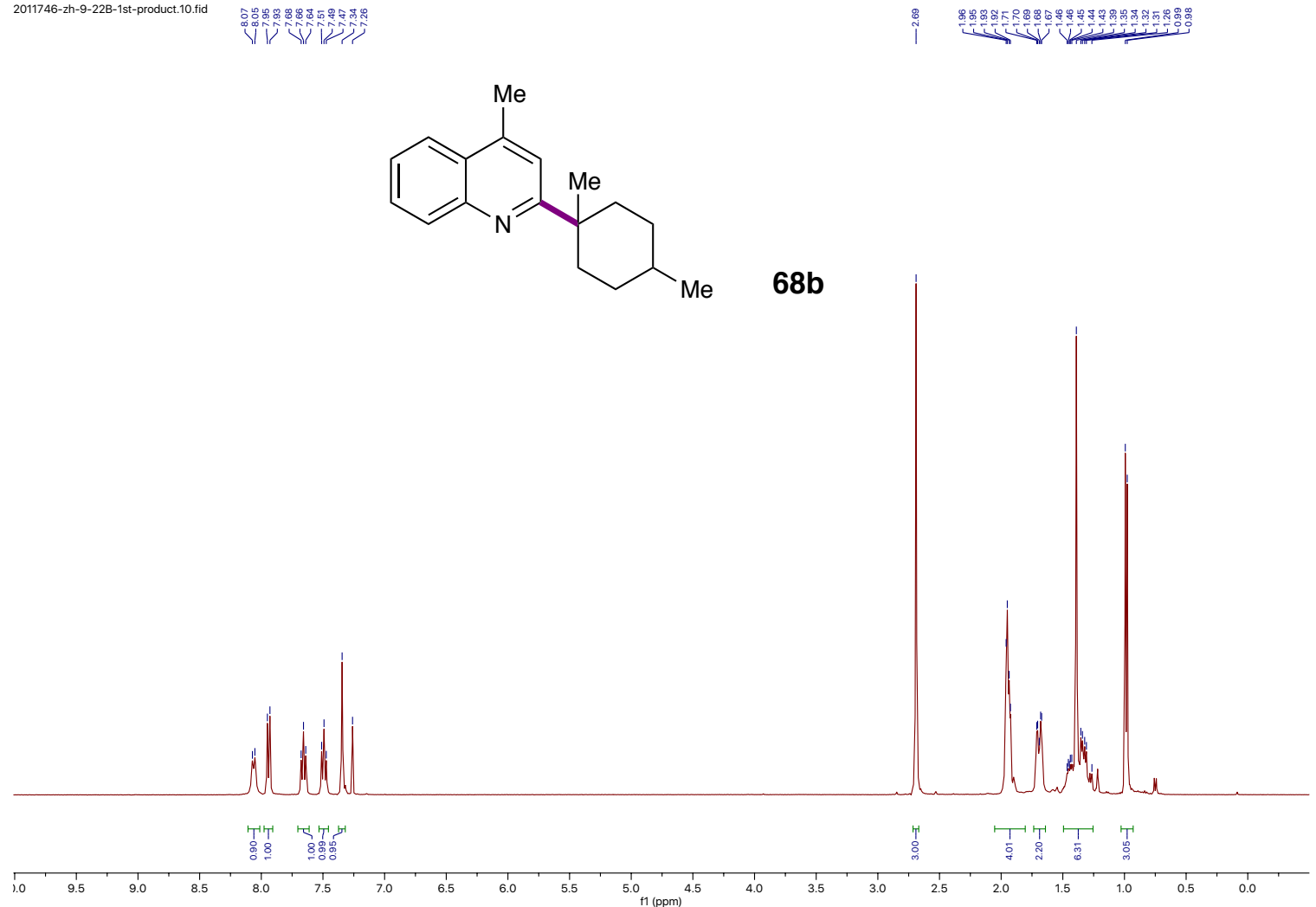

zh-9-22B-1st
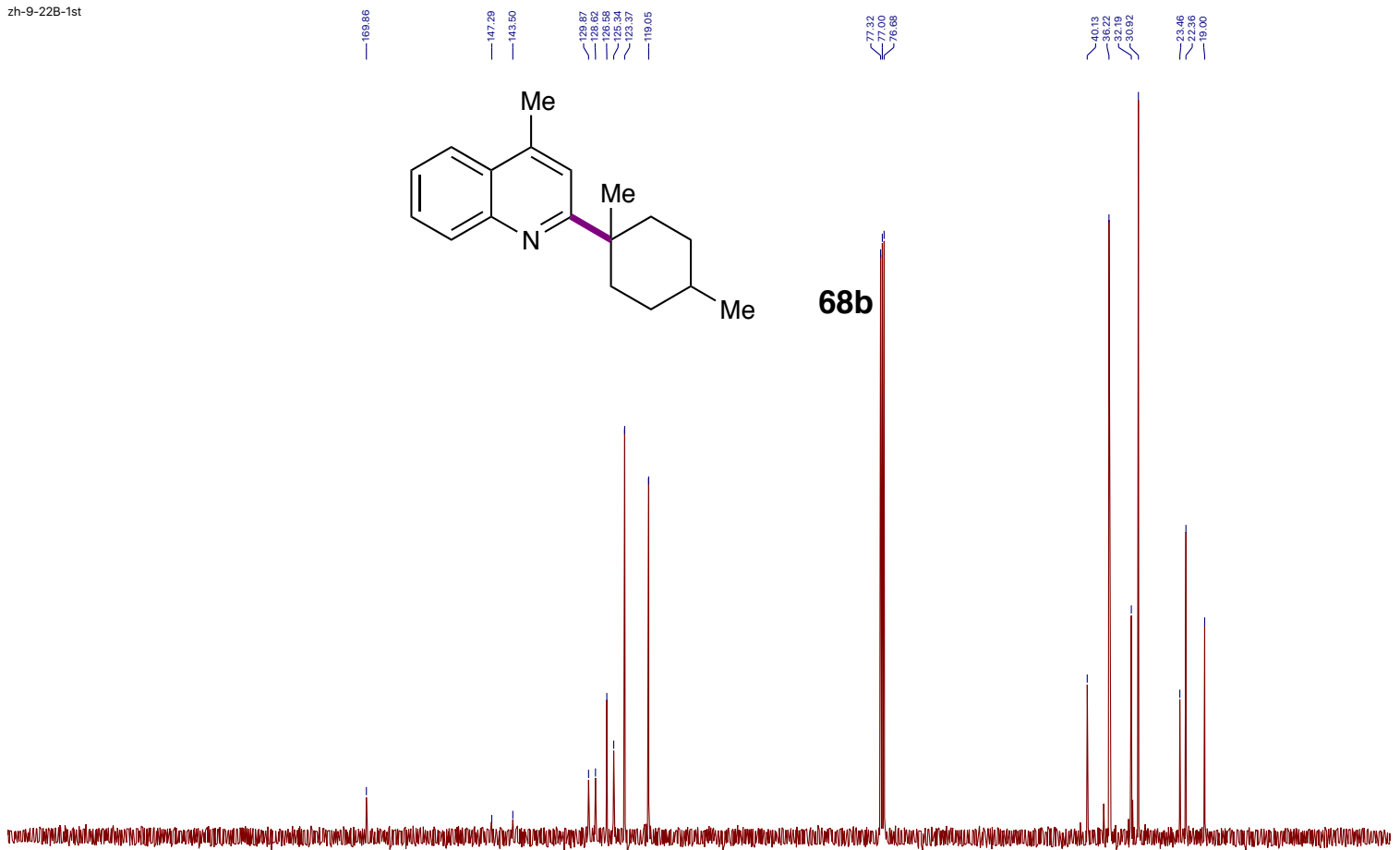

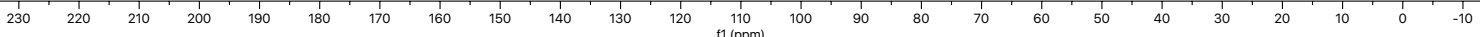



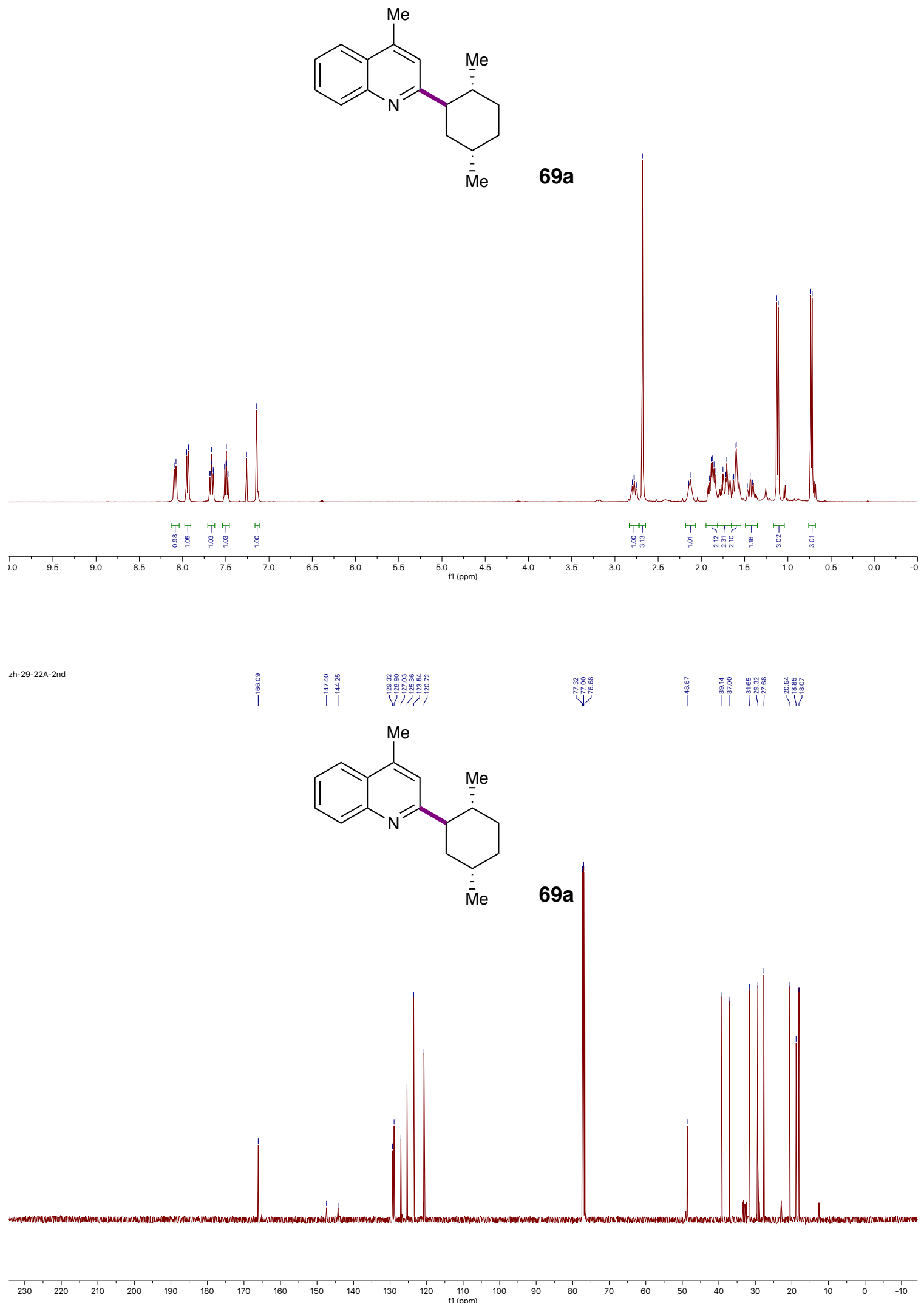

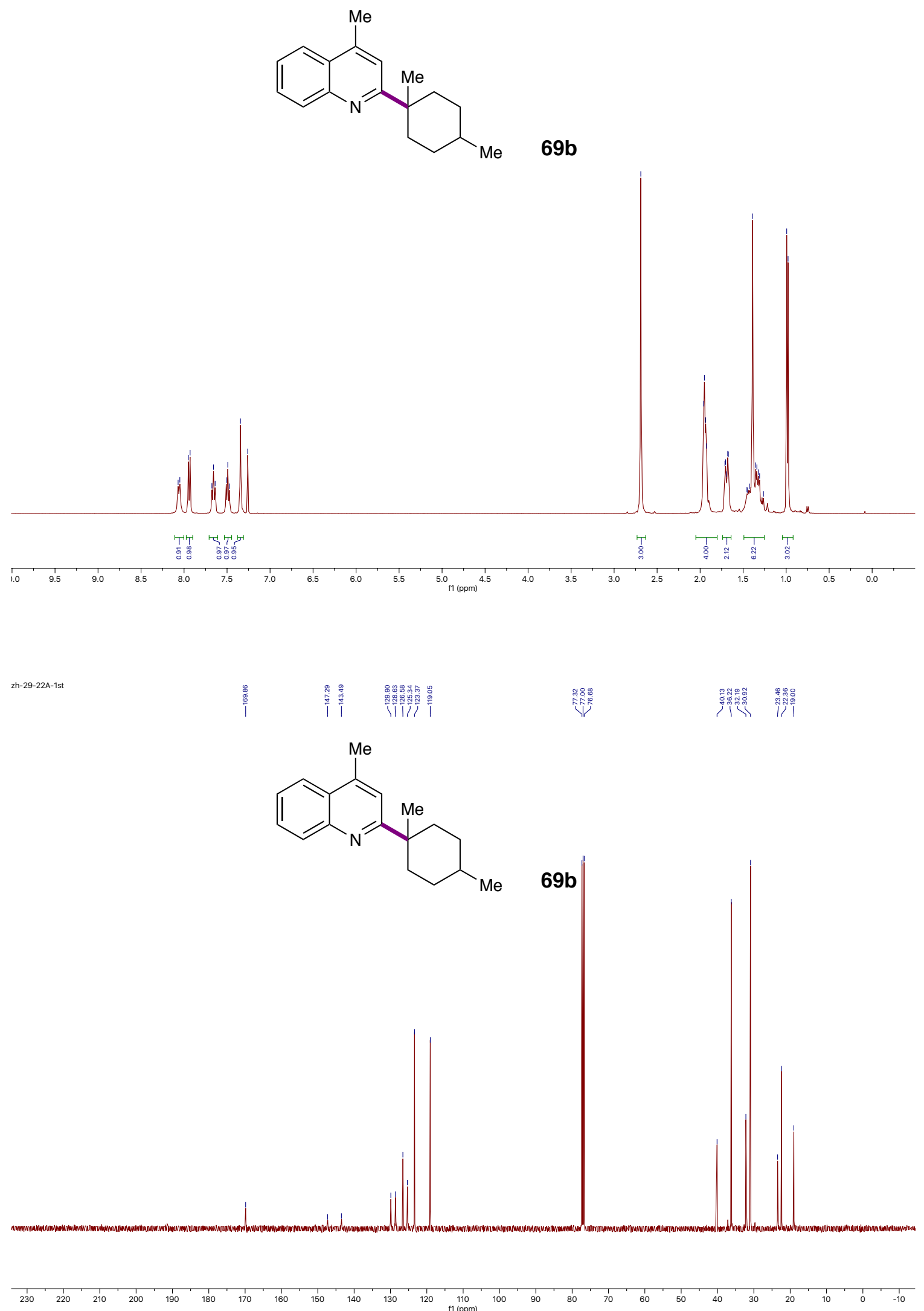


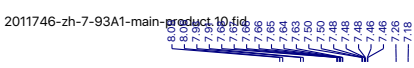<smiles>Cc1cc([C@@H]2C[C@H]3C[C@@H]2C[C@H]3O)nc2ccccc12</smiles>

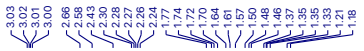

70

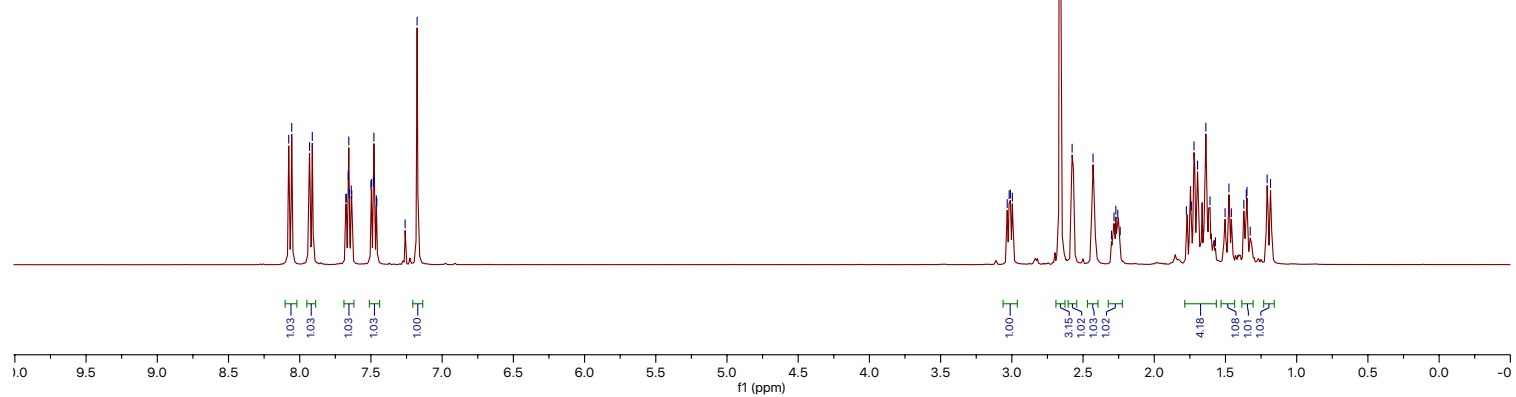

zh-7-93A1

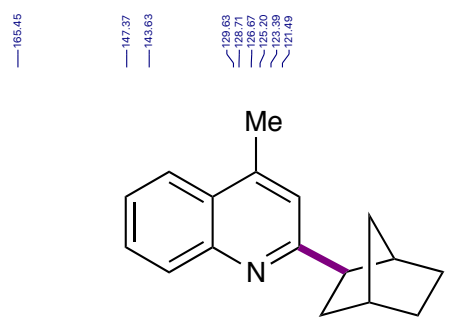

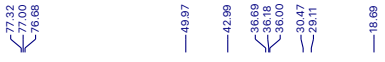

70

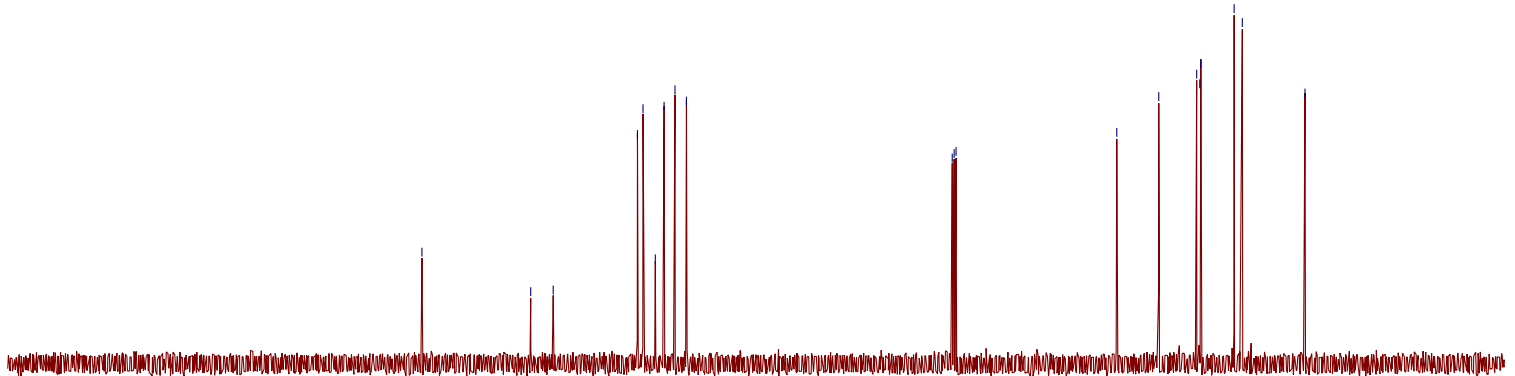

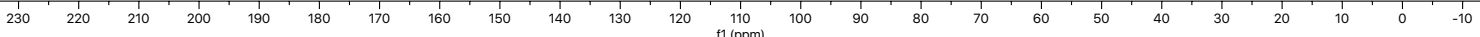


<smiles>CCCCc1cc([N+](=O)[O-])c2ccccc2n1</smiles><smiles>Cc1cc(C(C)CC[18OH])nc2ccccc12</smiles><smiles>CCC(c1cc([N+](=O)[O-])c2ccccc2n1)C(C)C</smiles>
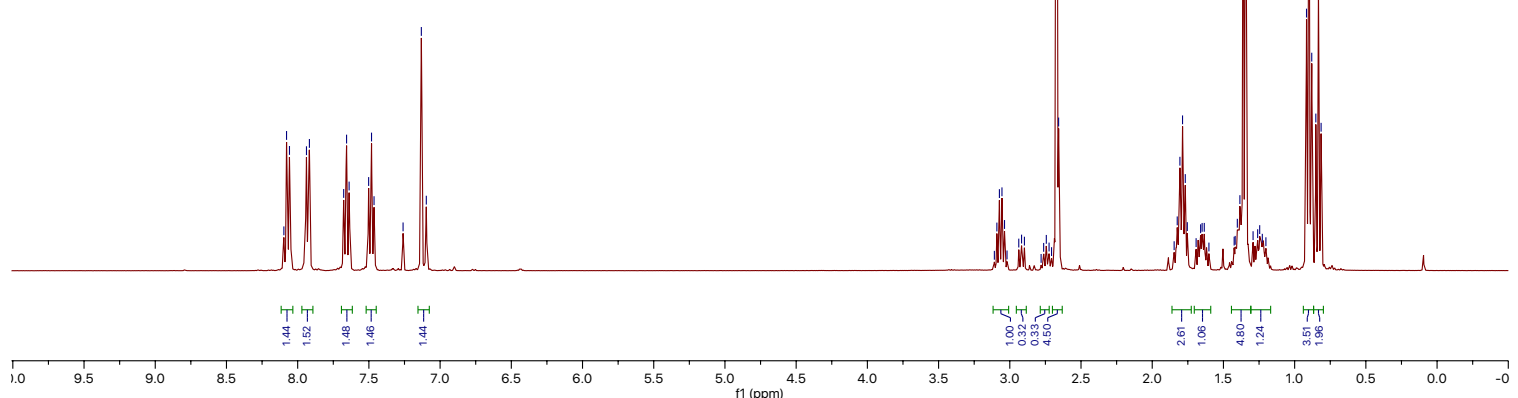<smiles>CCCCc1cc(C)c2ccccc2n1</smiles><smiles>CCCC(C)c1cc([N+](=O)[O-])c2ccccc2n1</smiles><smiles>CCC(CC)c1cc([N+](C)([O-])[O-])c2ccccc2n1</smiles><smiles>C#CC#CC(=C)C(C)CCCC</smiles> 

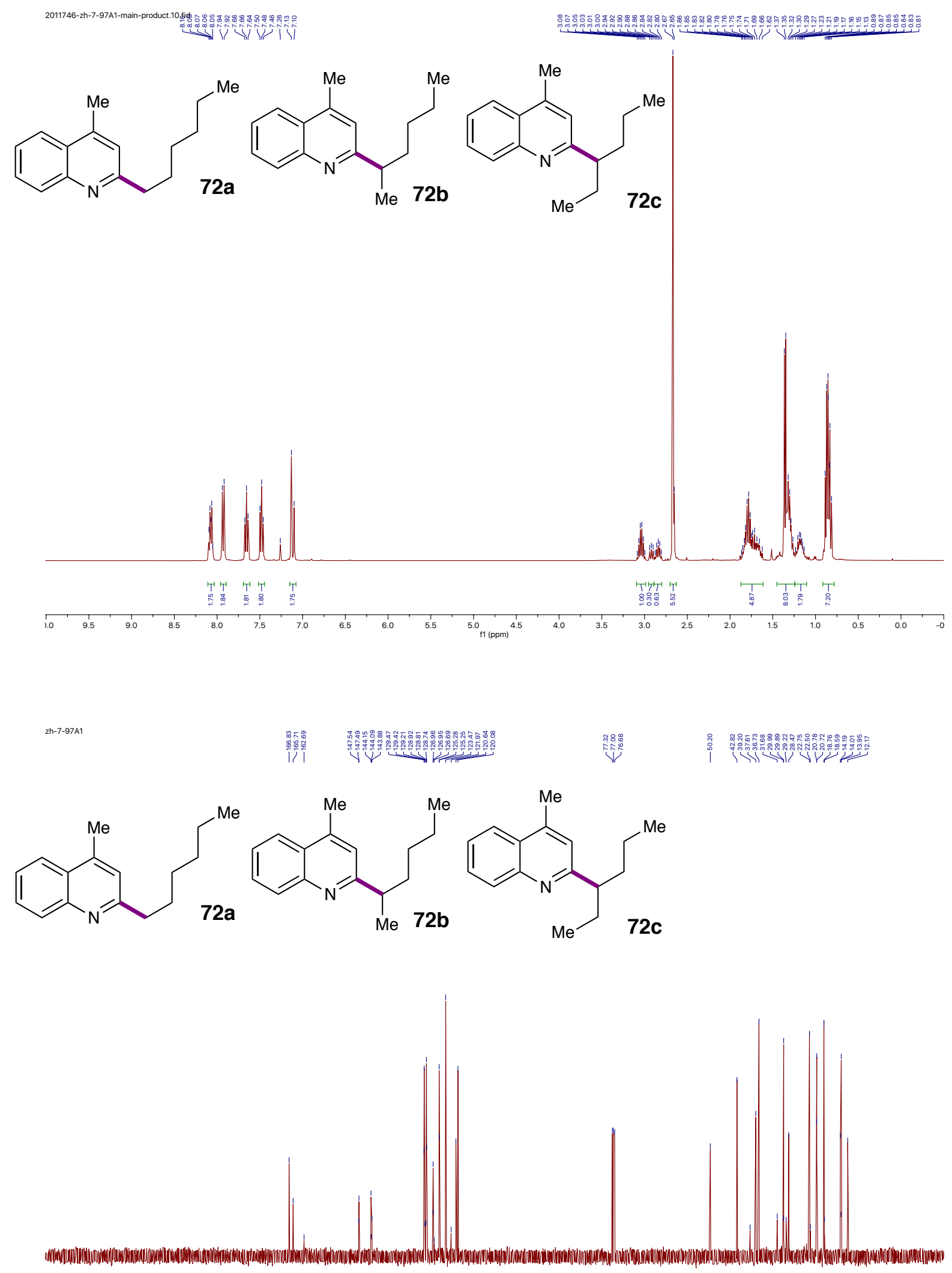

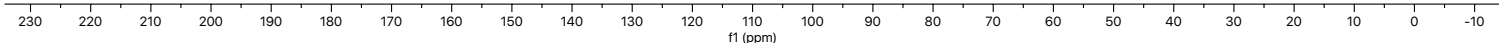



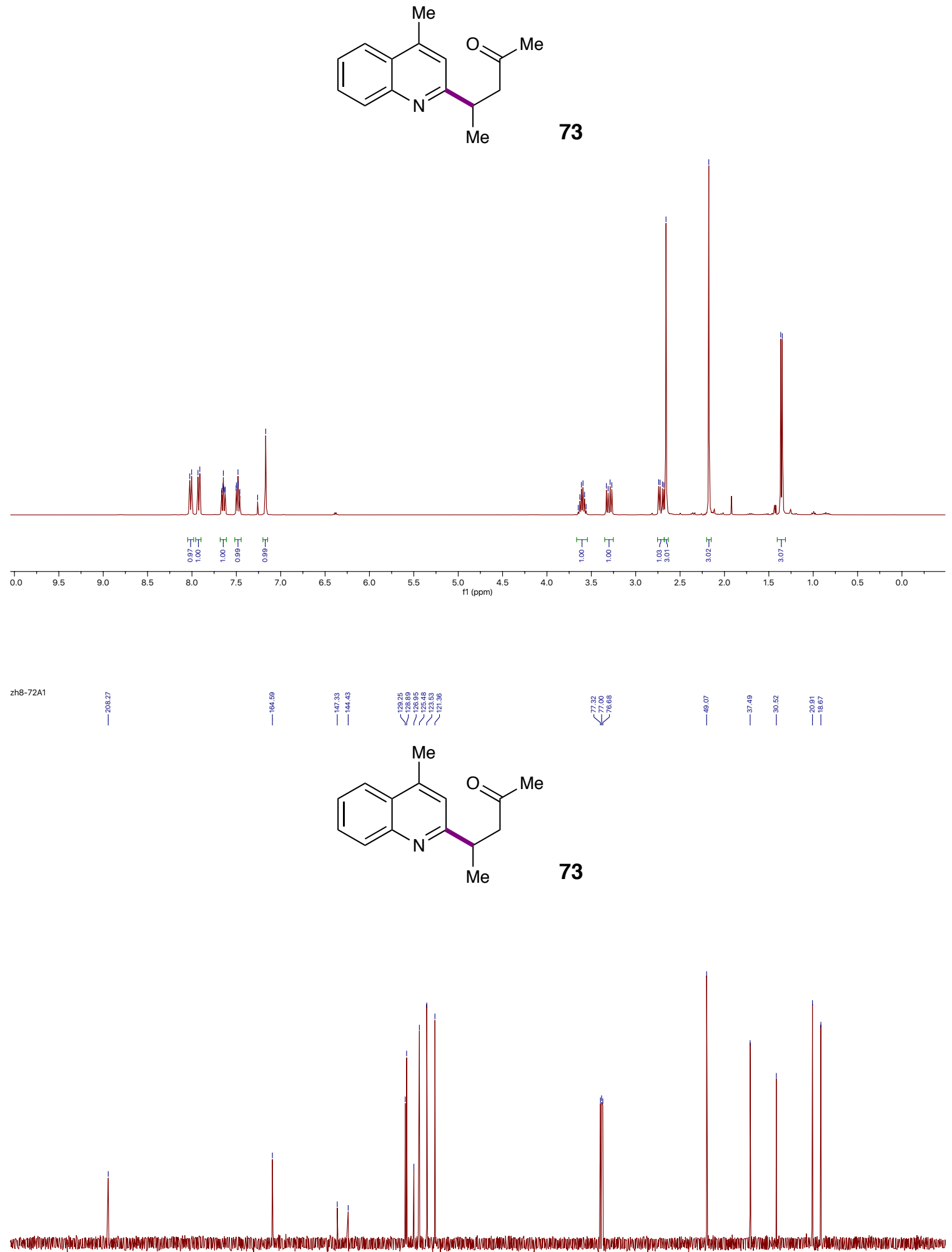

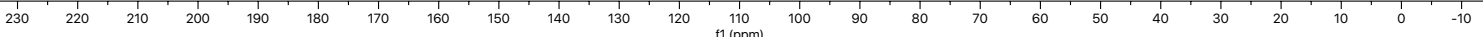



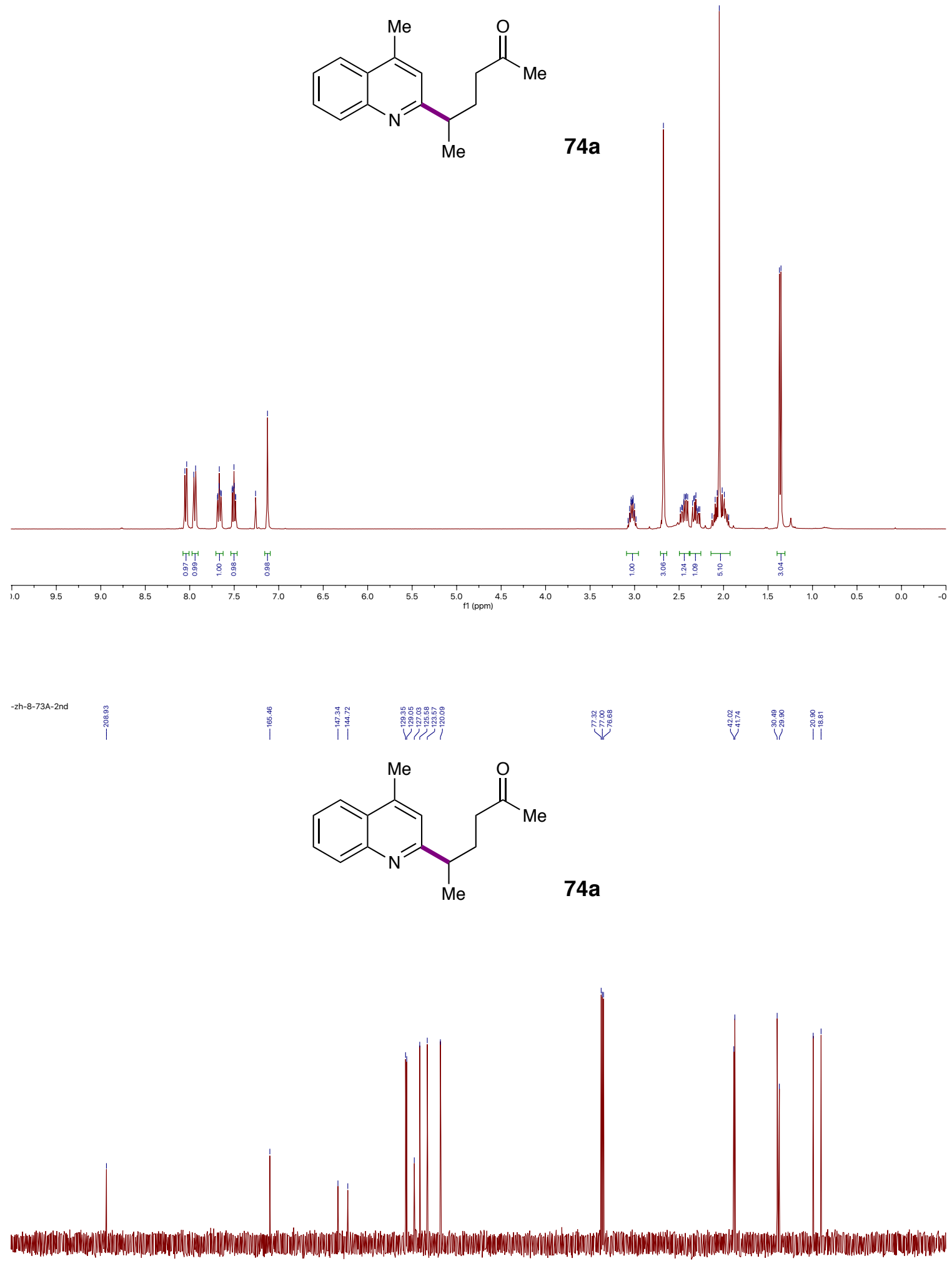

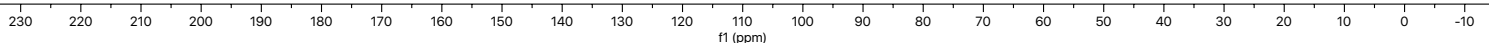



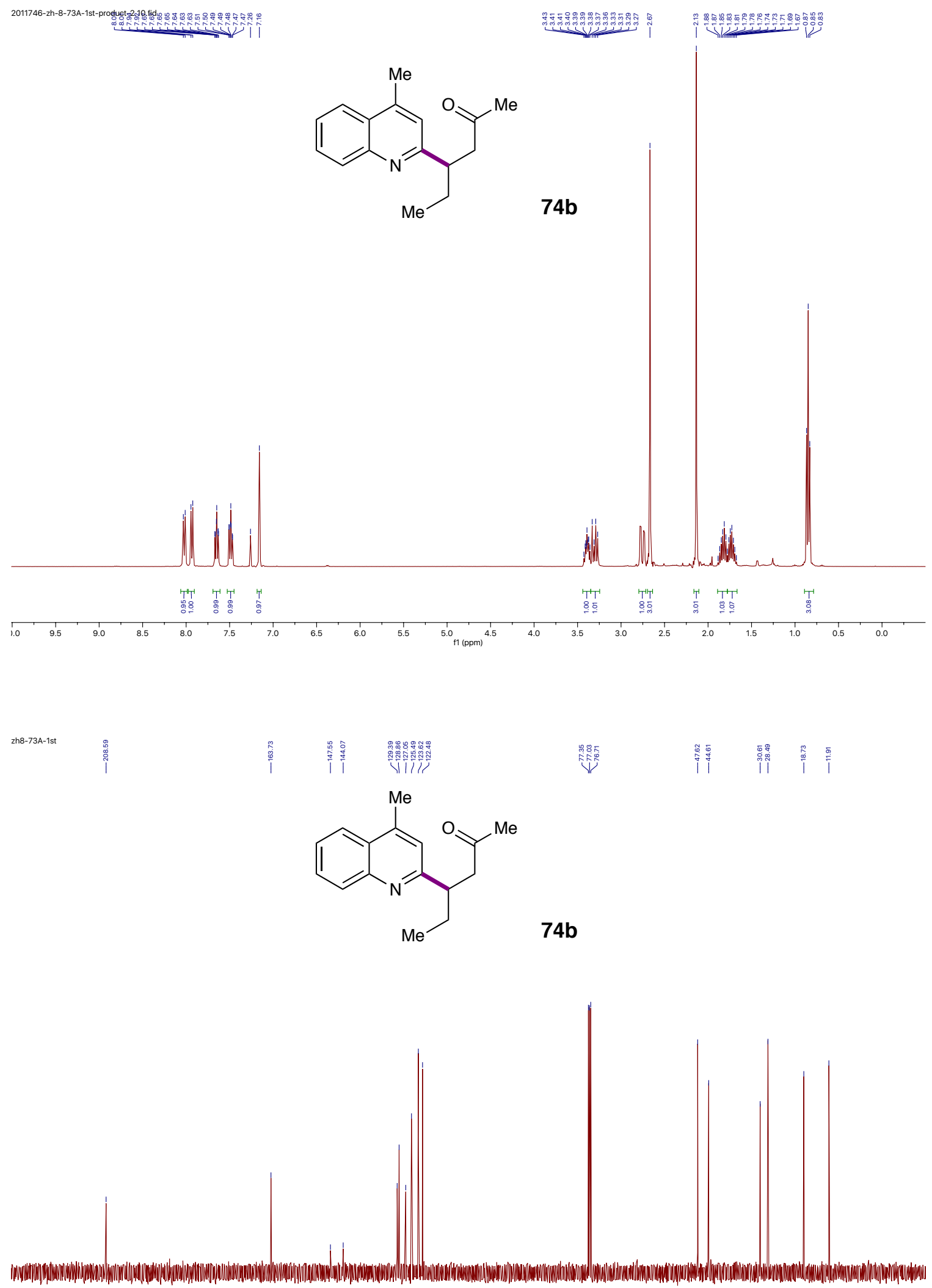

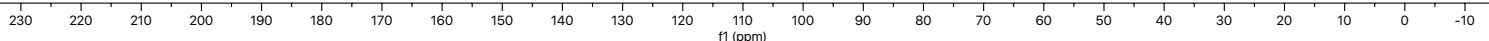



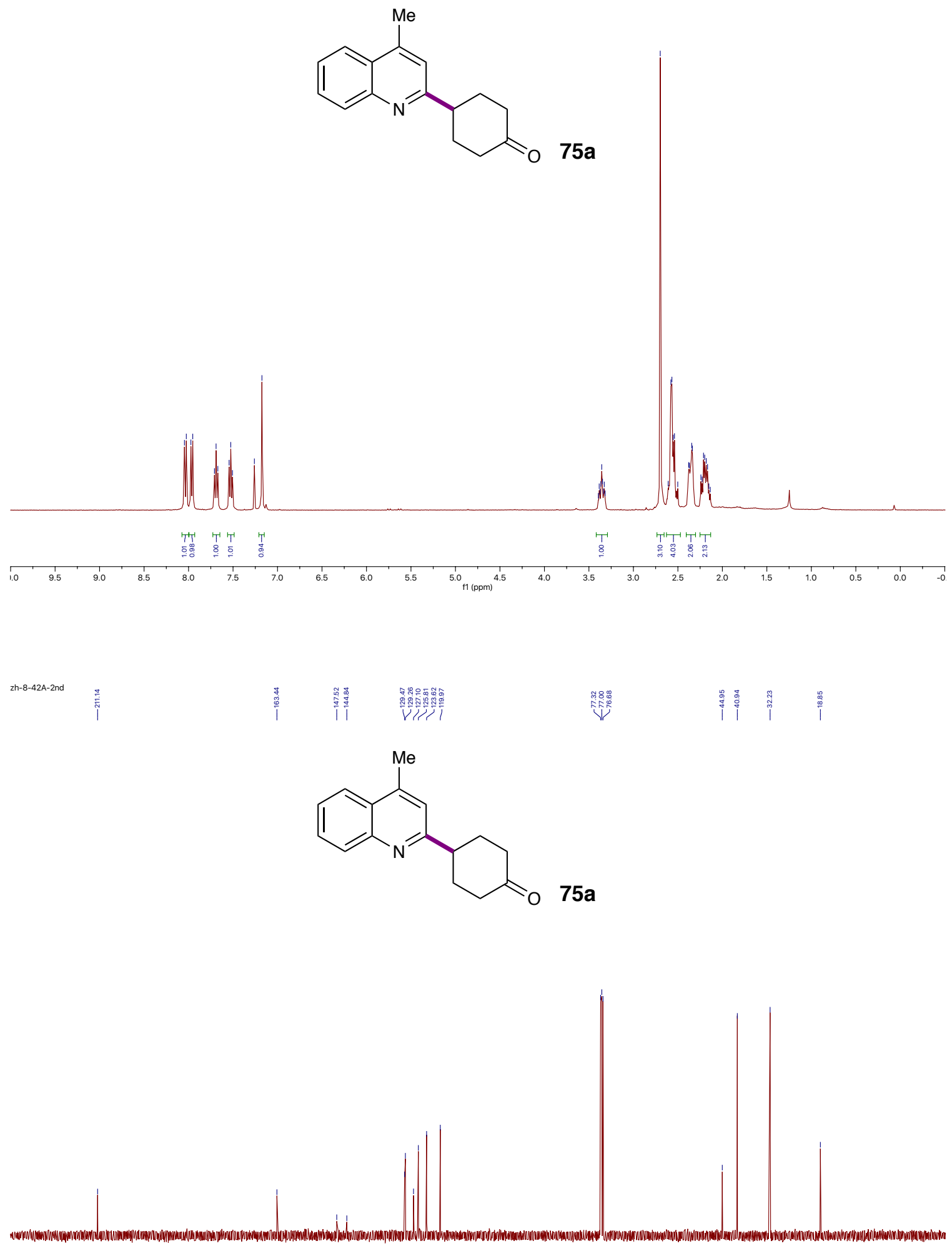

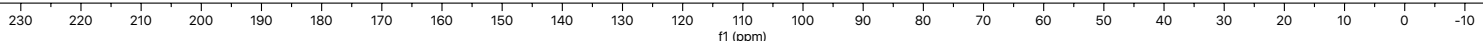


<smiles>Cc1cc(C2CCCC(=O)C2)nc2ccccc12</smiles>

$75 b$
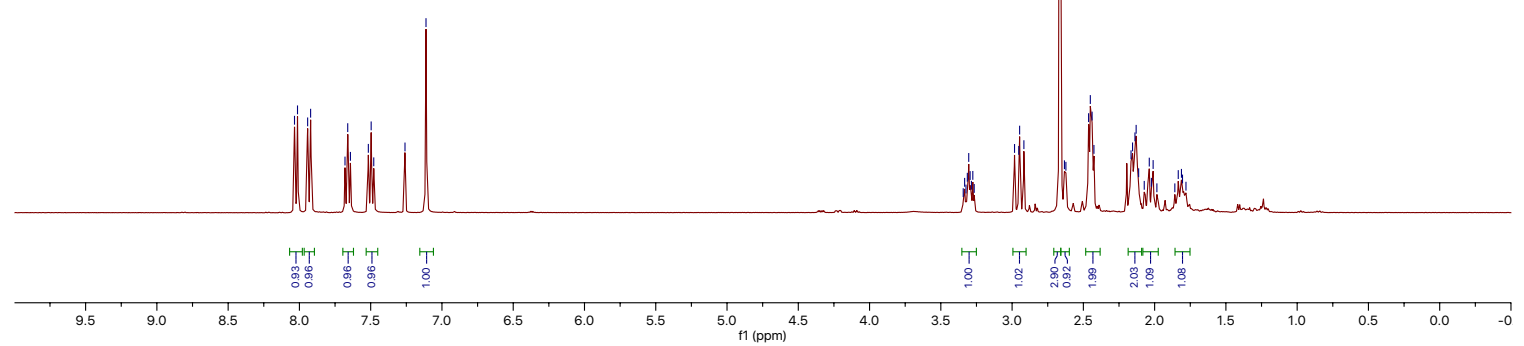

zh8-42

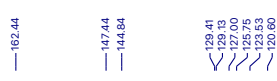

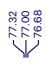

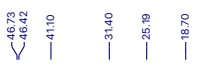<smiles>O=C1CCCC(c2cc([N+](=O)[O-])c3ccccc3n2)C1</smiles>

$75 b$

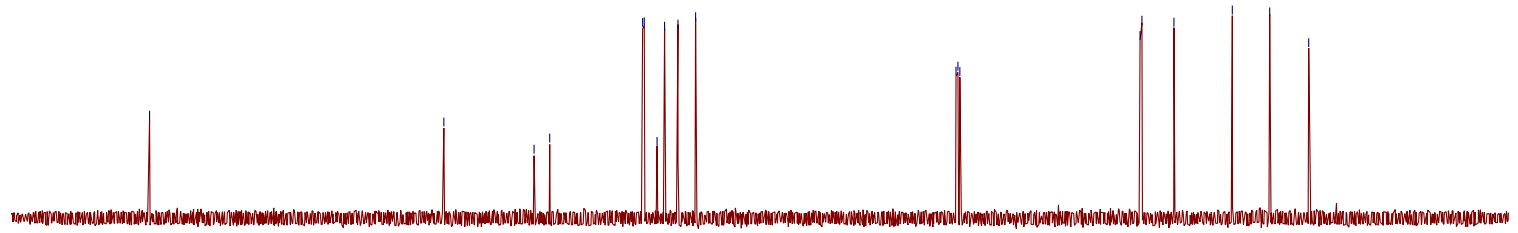

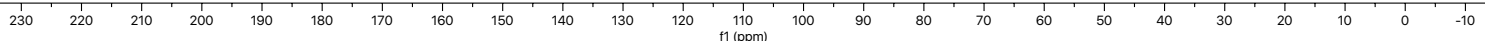



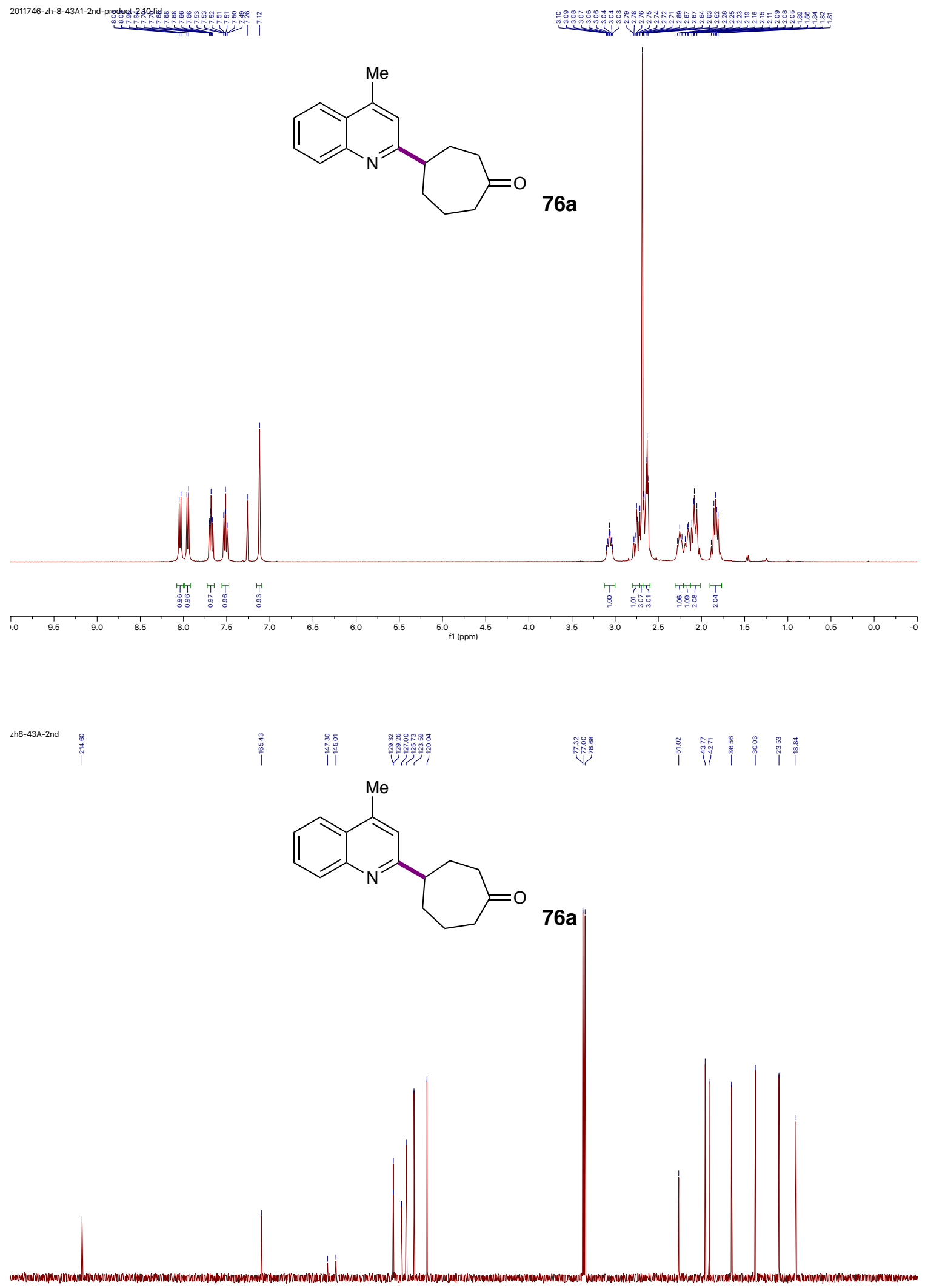

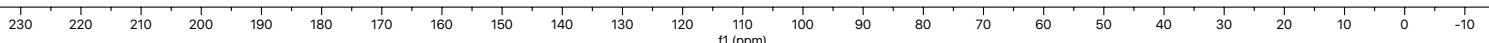


<smiles>Cc1cc(C2CCCCC(=O)C2)nc2ccccc12</smiles>

76b

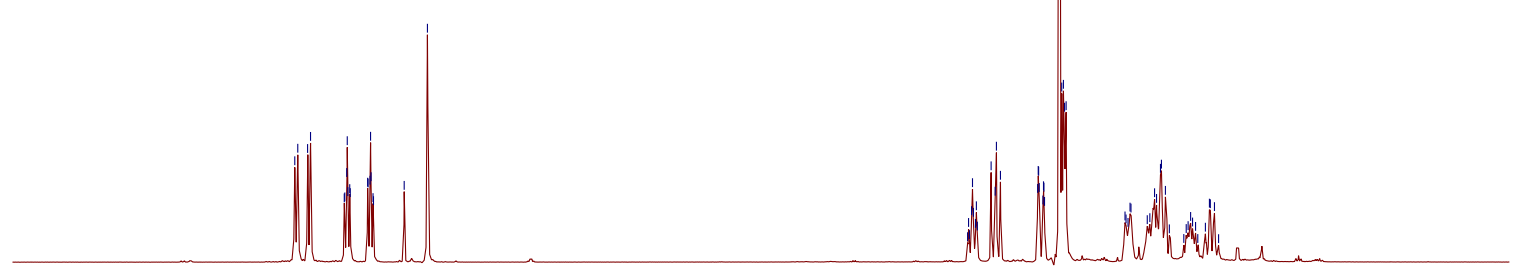

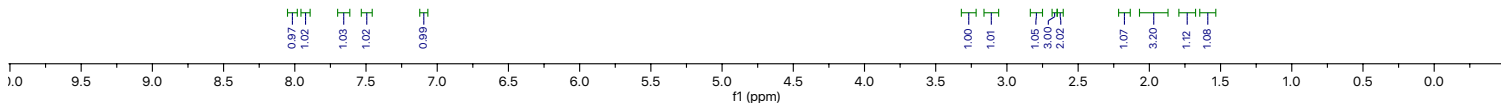
zh8-43a-1st<smiles>Cc1cc(C2CCCCC(=O)C2)nc2ccccc12</smiles>

76b

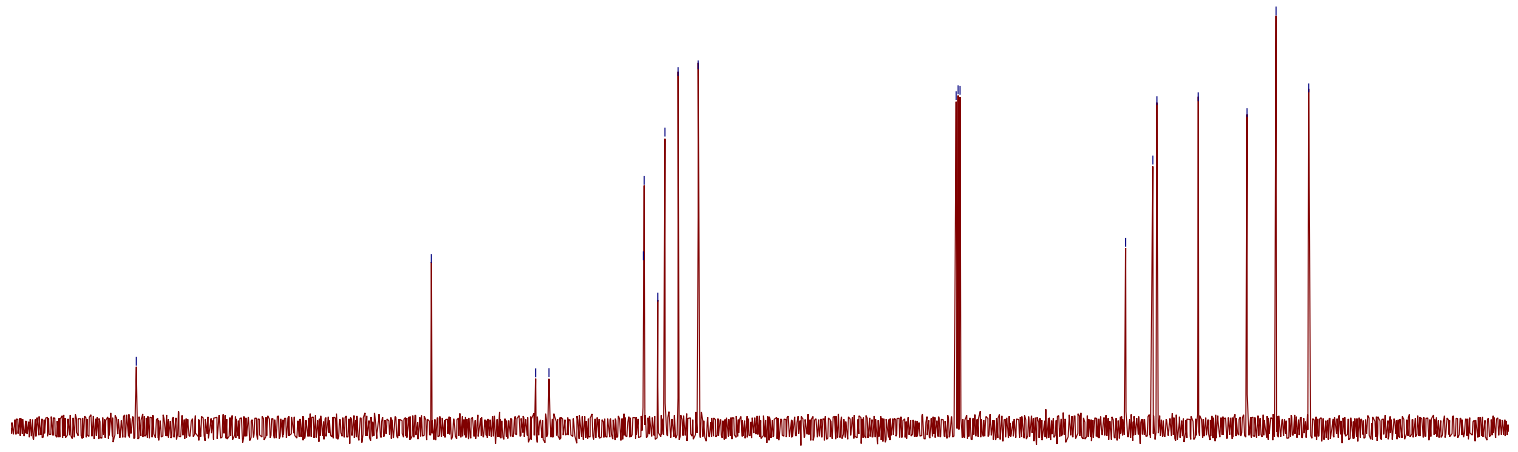

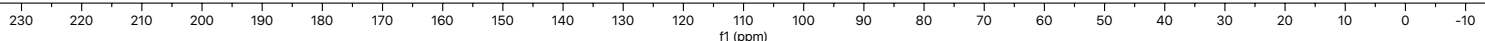


2011746-zh-8-85A-2nd-part1 10 fid

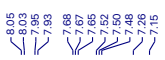

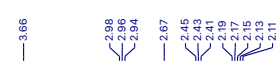<smiles>COC(=O)CCCc1cc(C)c2ccccc2n1</smiles>

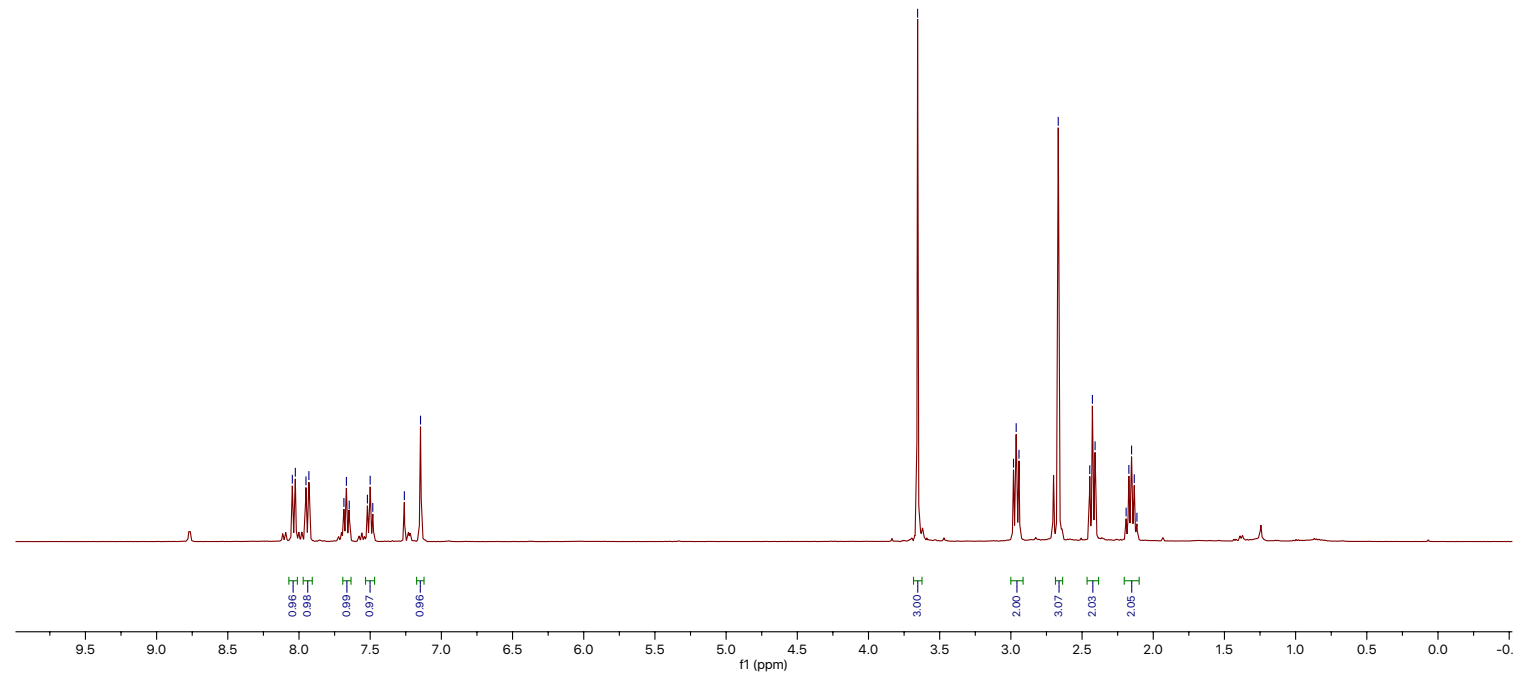

$-2 h-8-85 \mathrm{~A}-2$ nd

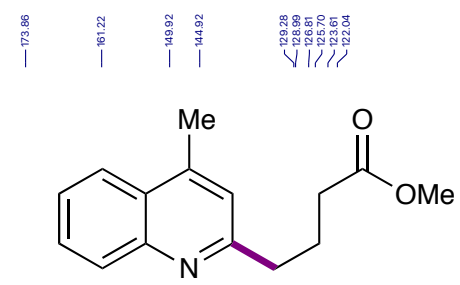

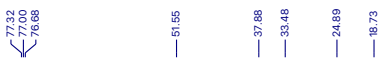

$77 a$

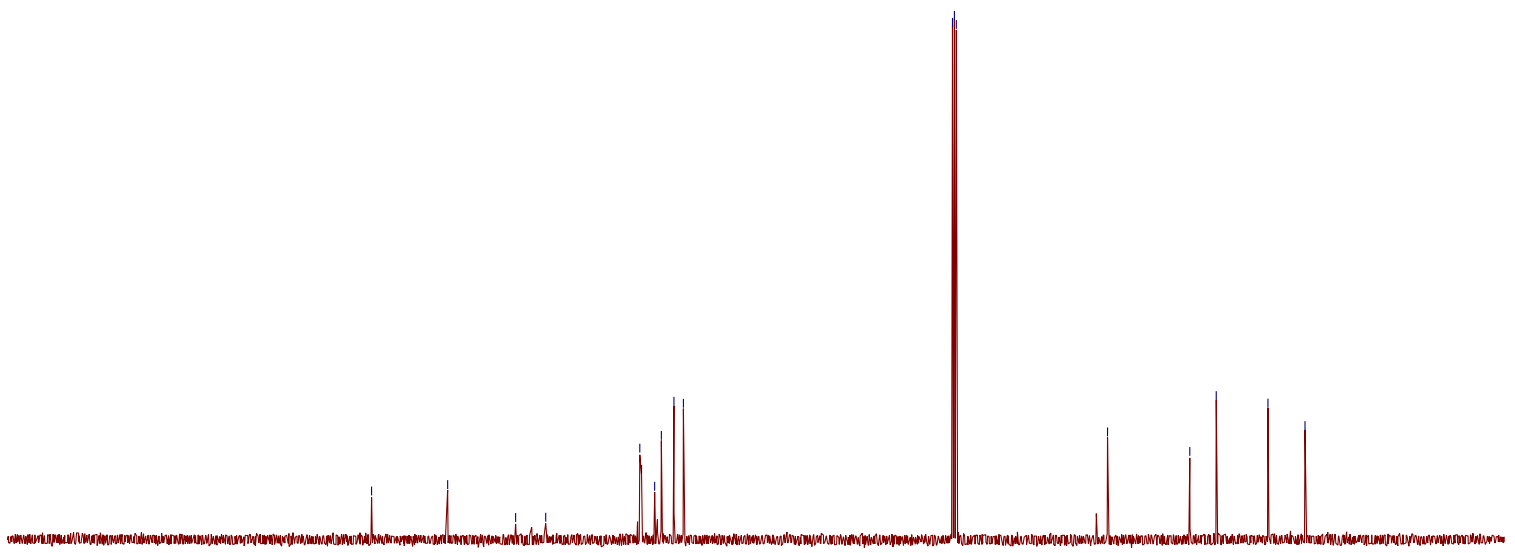

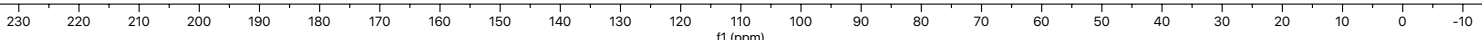



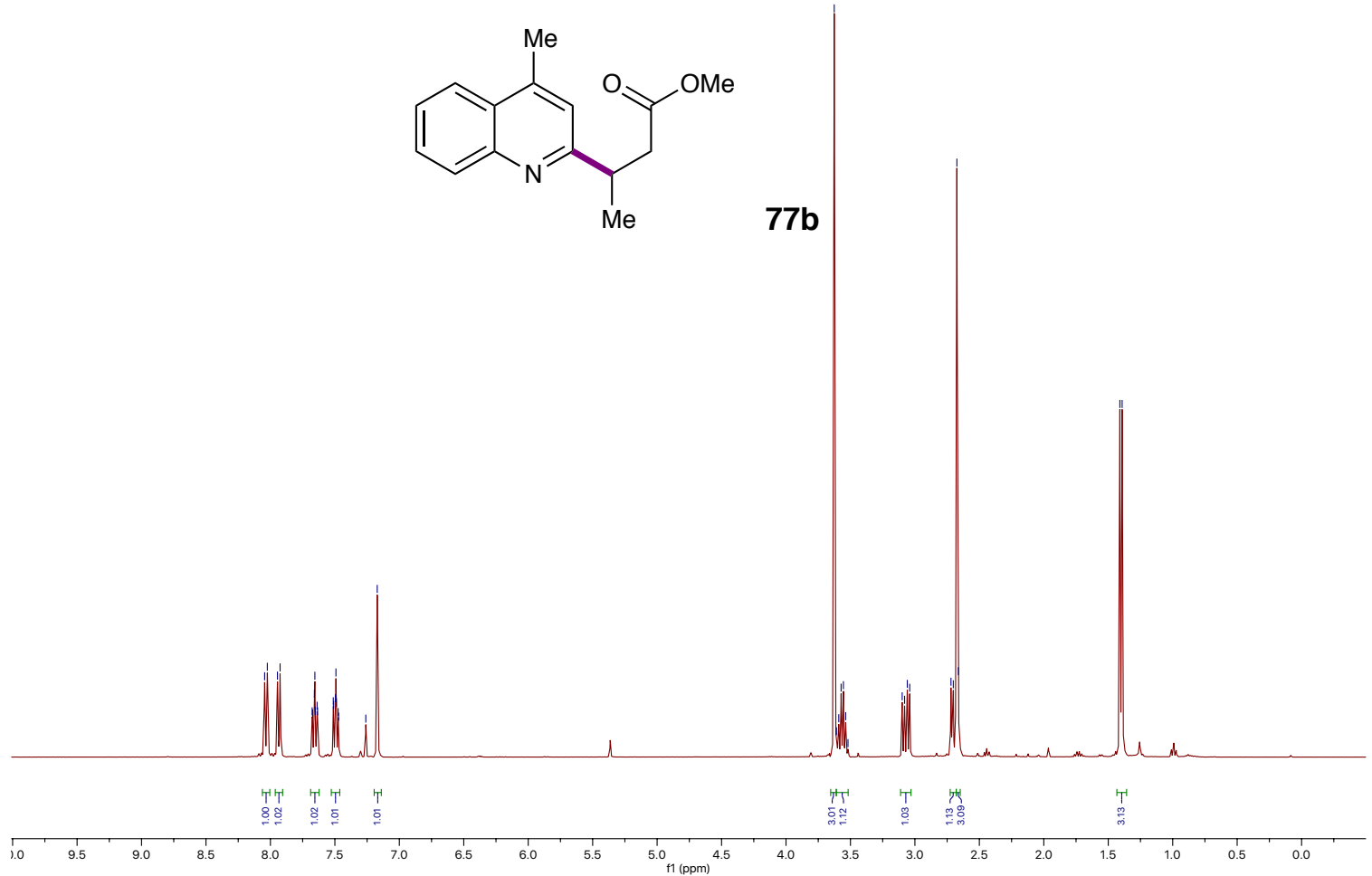
zh8-85A-1st<smiles>COC(=O)C[C@H](C)c1cc(C)c2ccccc2n1</smiles>

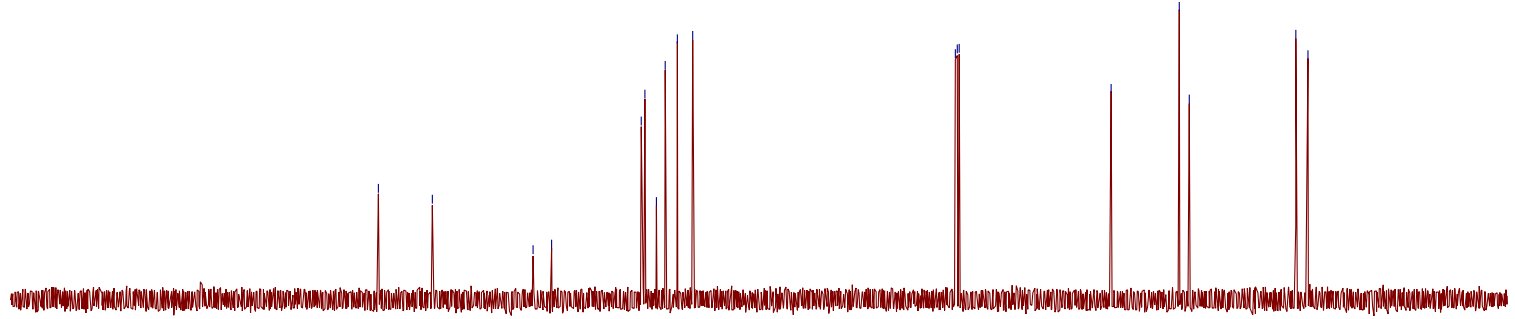

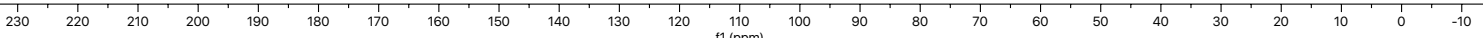



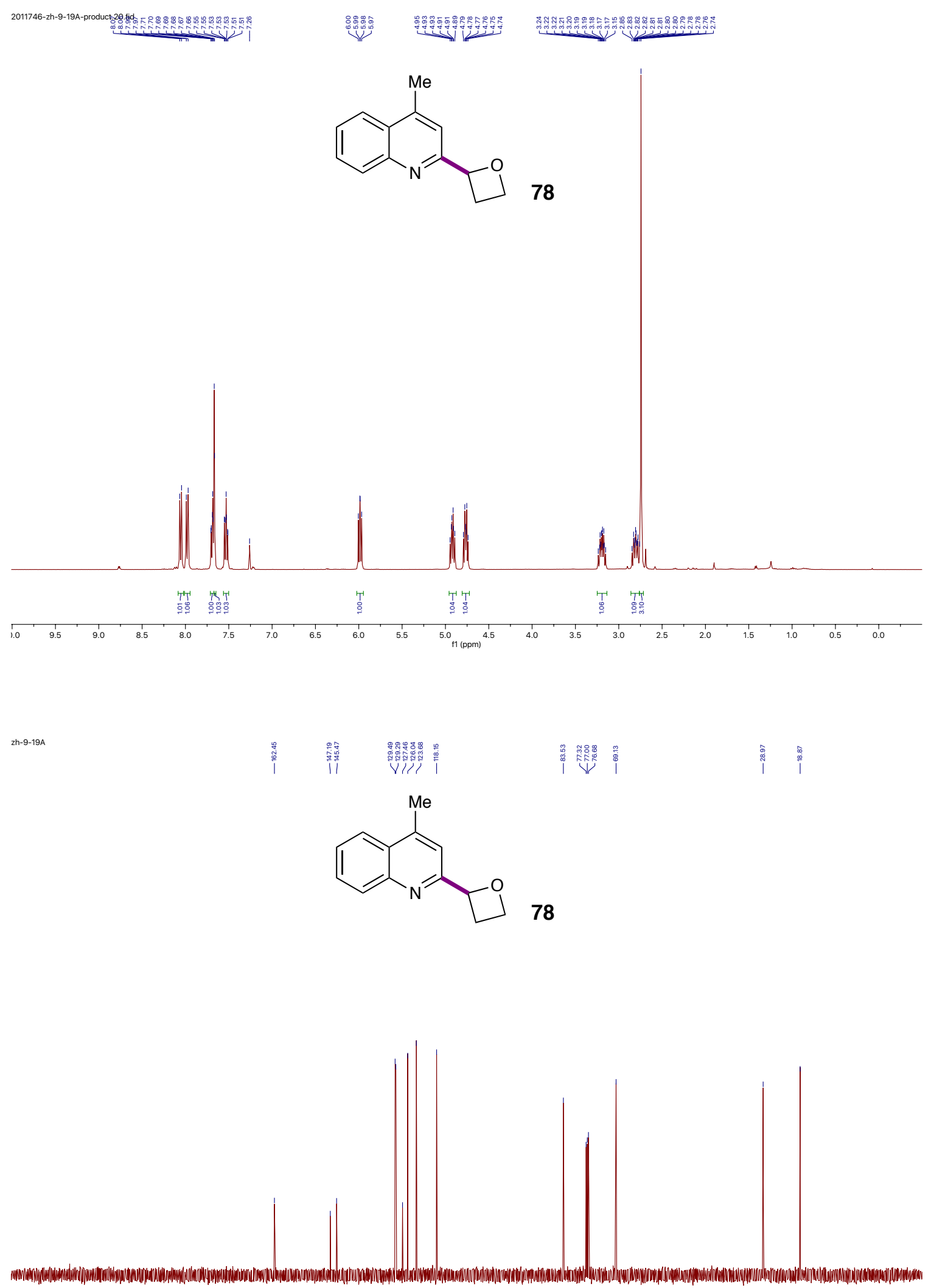

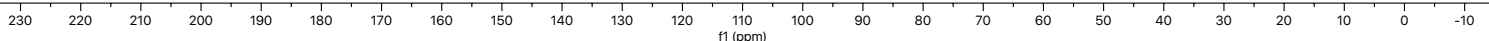




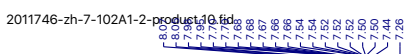

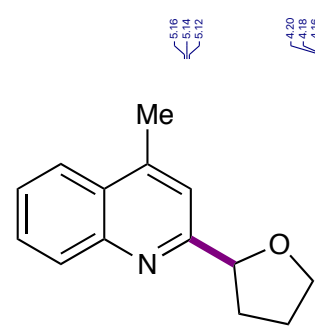

79

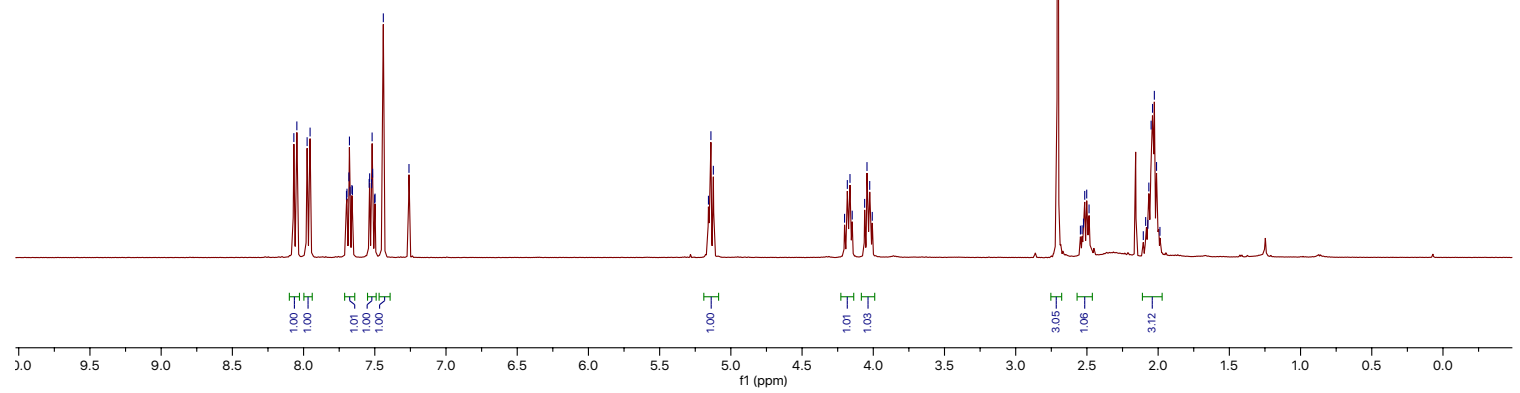

$-2 h-7-102 A 1$

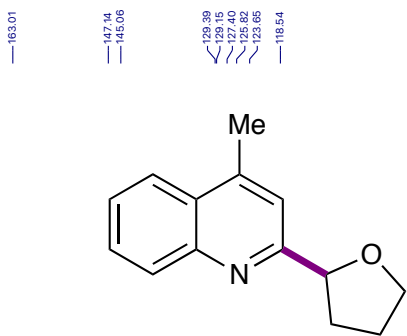

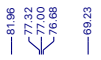

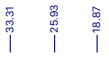

79

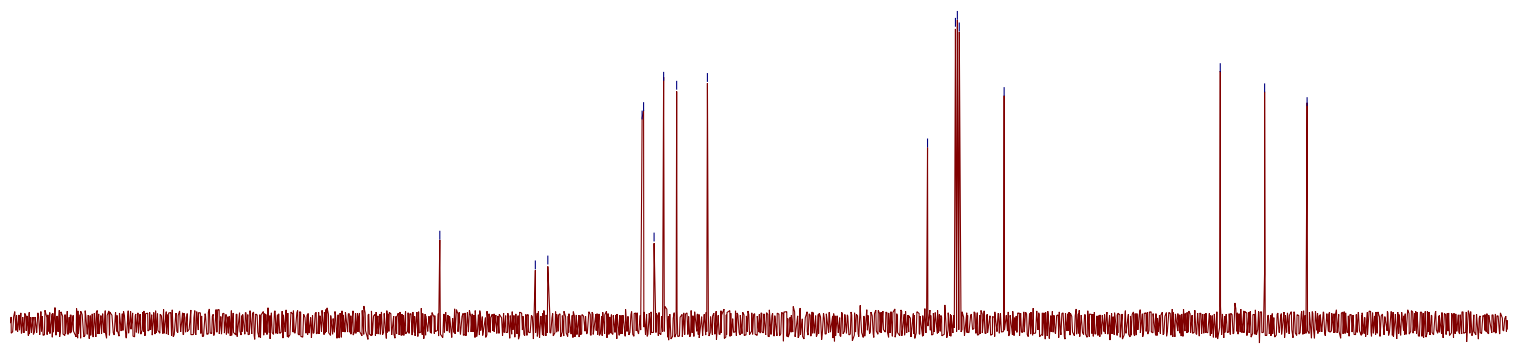

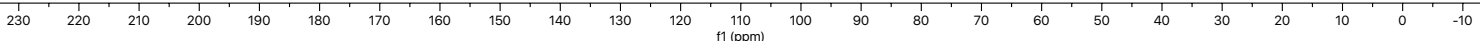



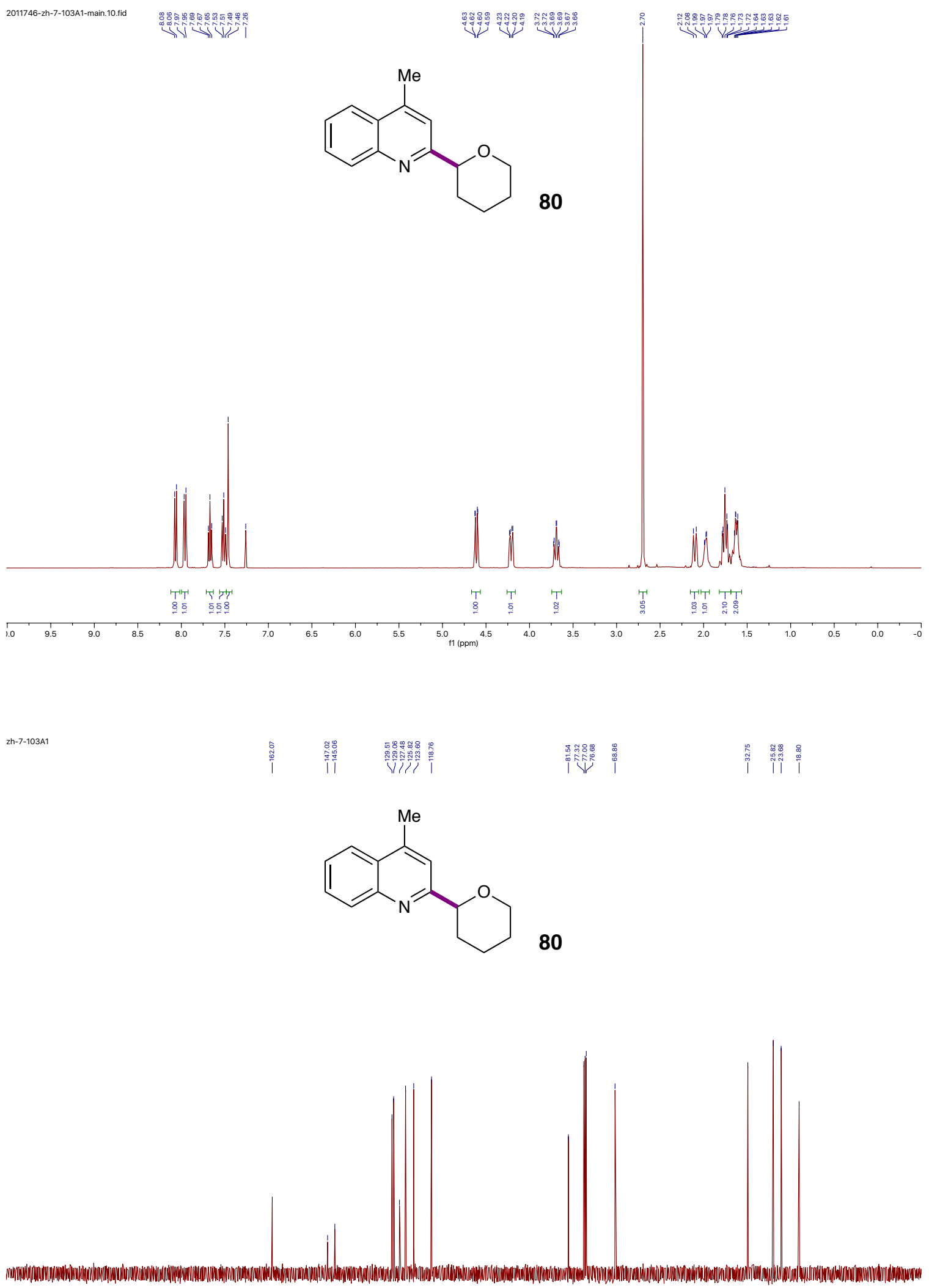

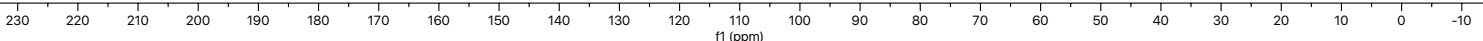




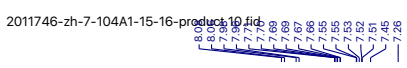
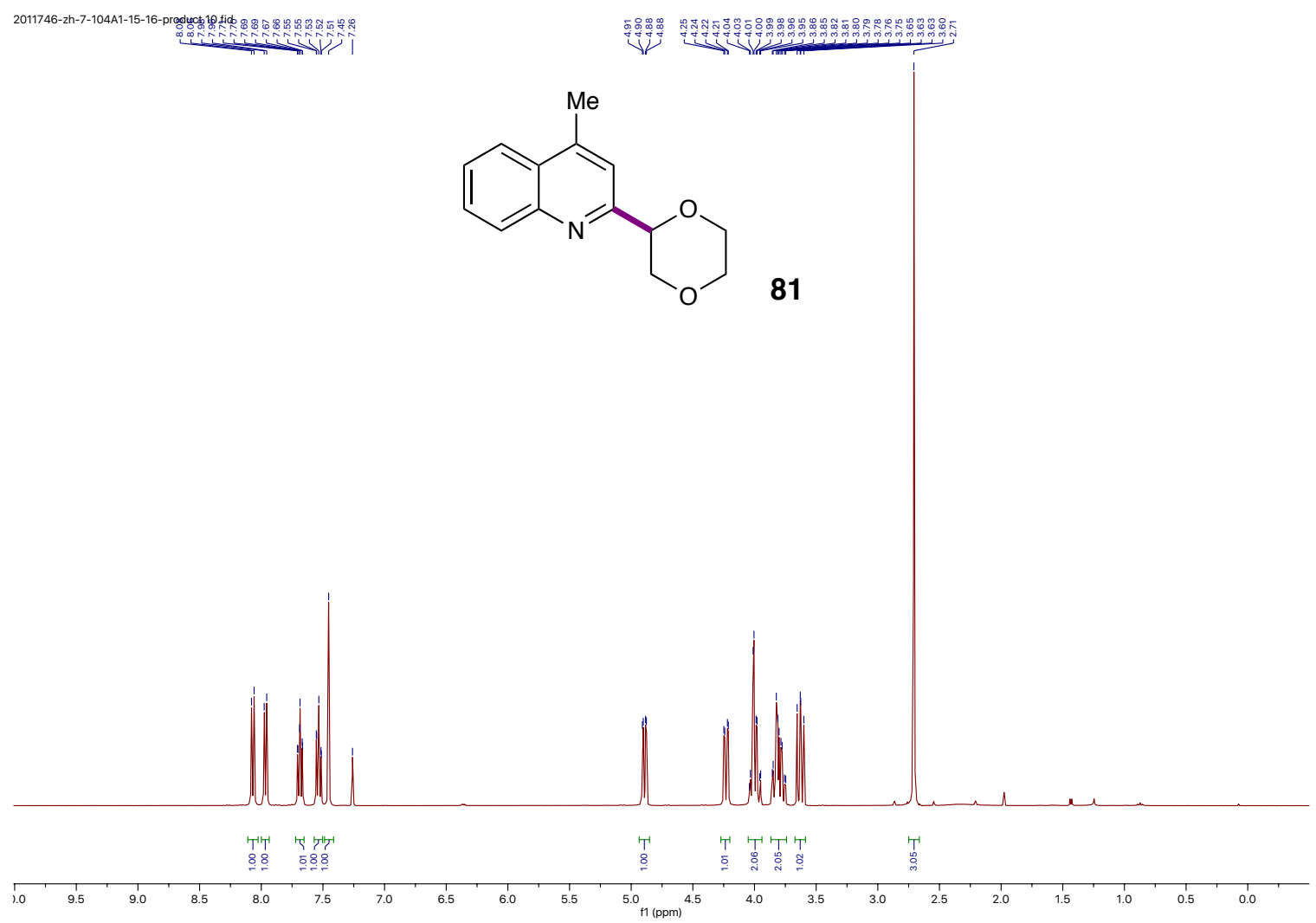

zh7-104A1

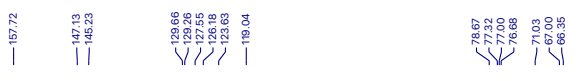

81

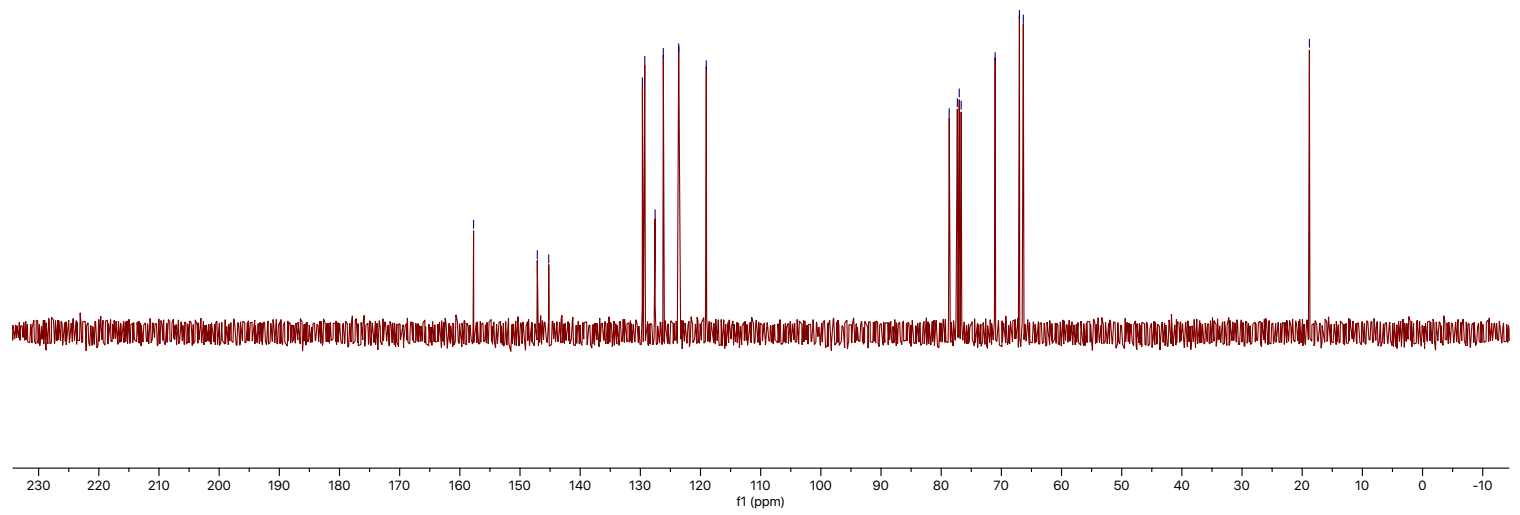


2011746-zh-9-20A-2.10.fid

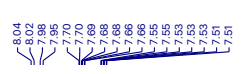

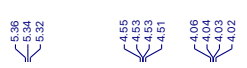

影

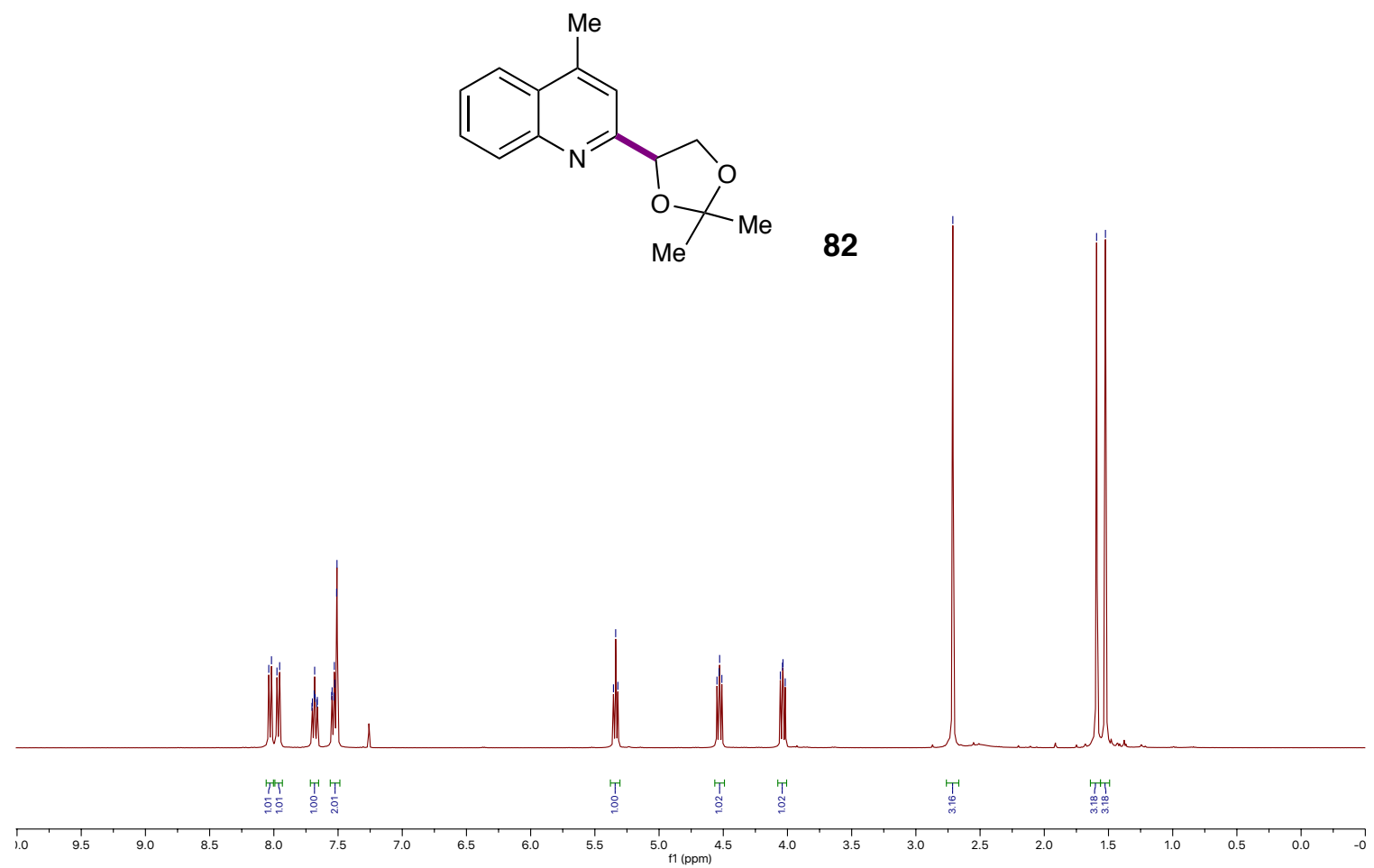

-zh-9-20A
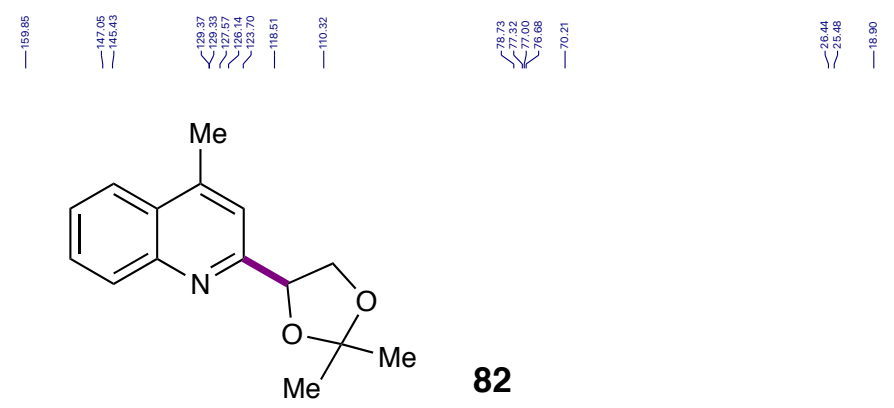

82

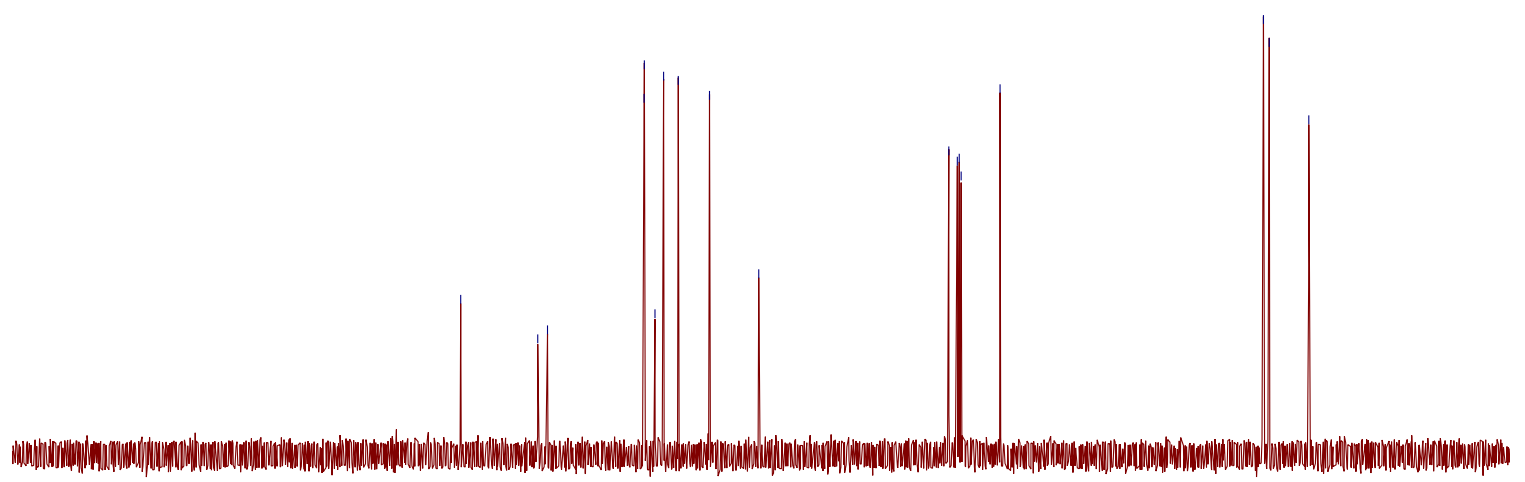

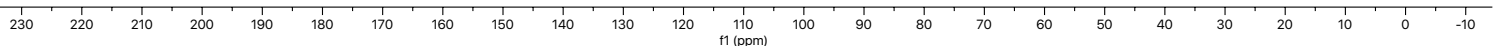




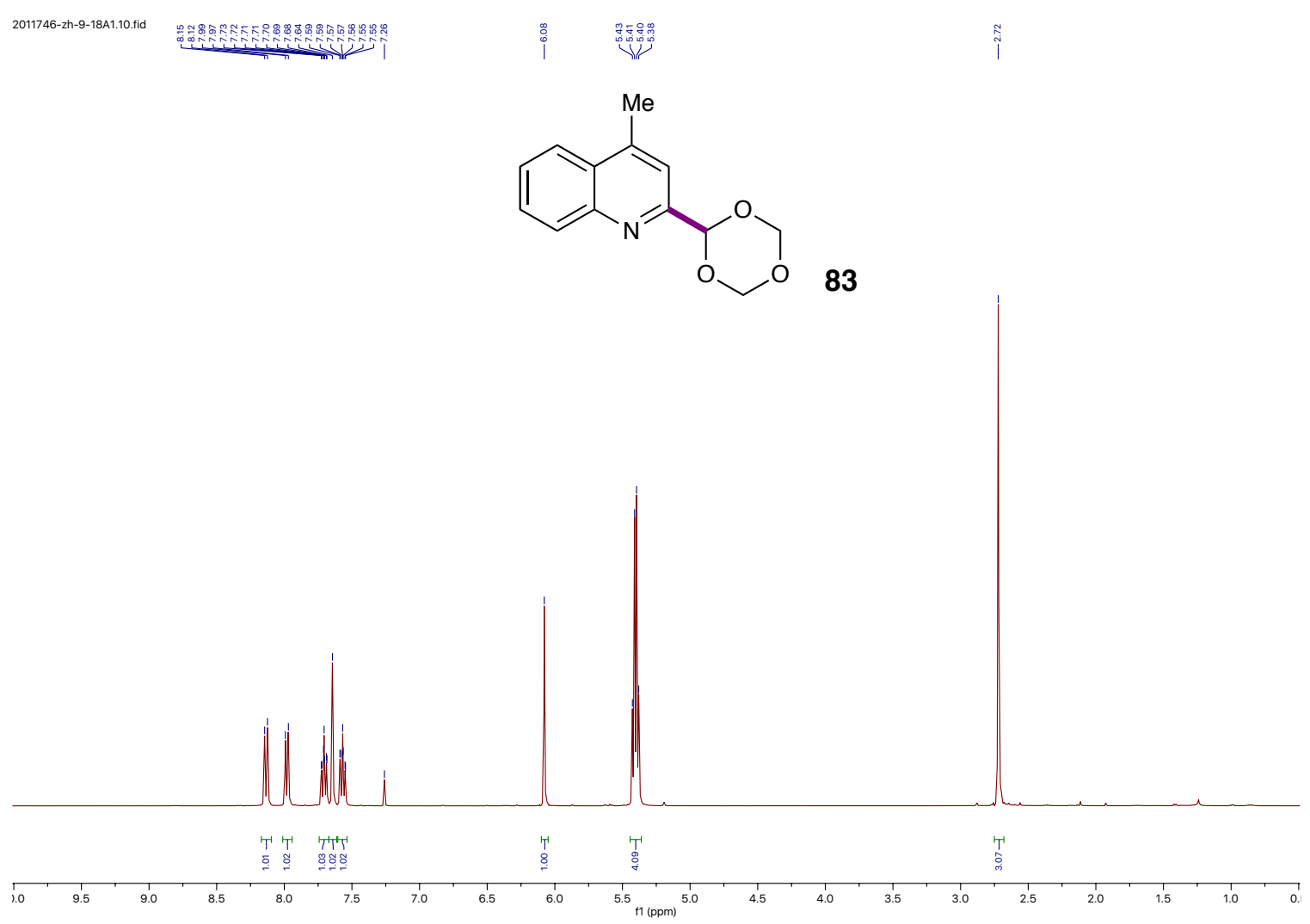

zh9-18A1
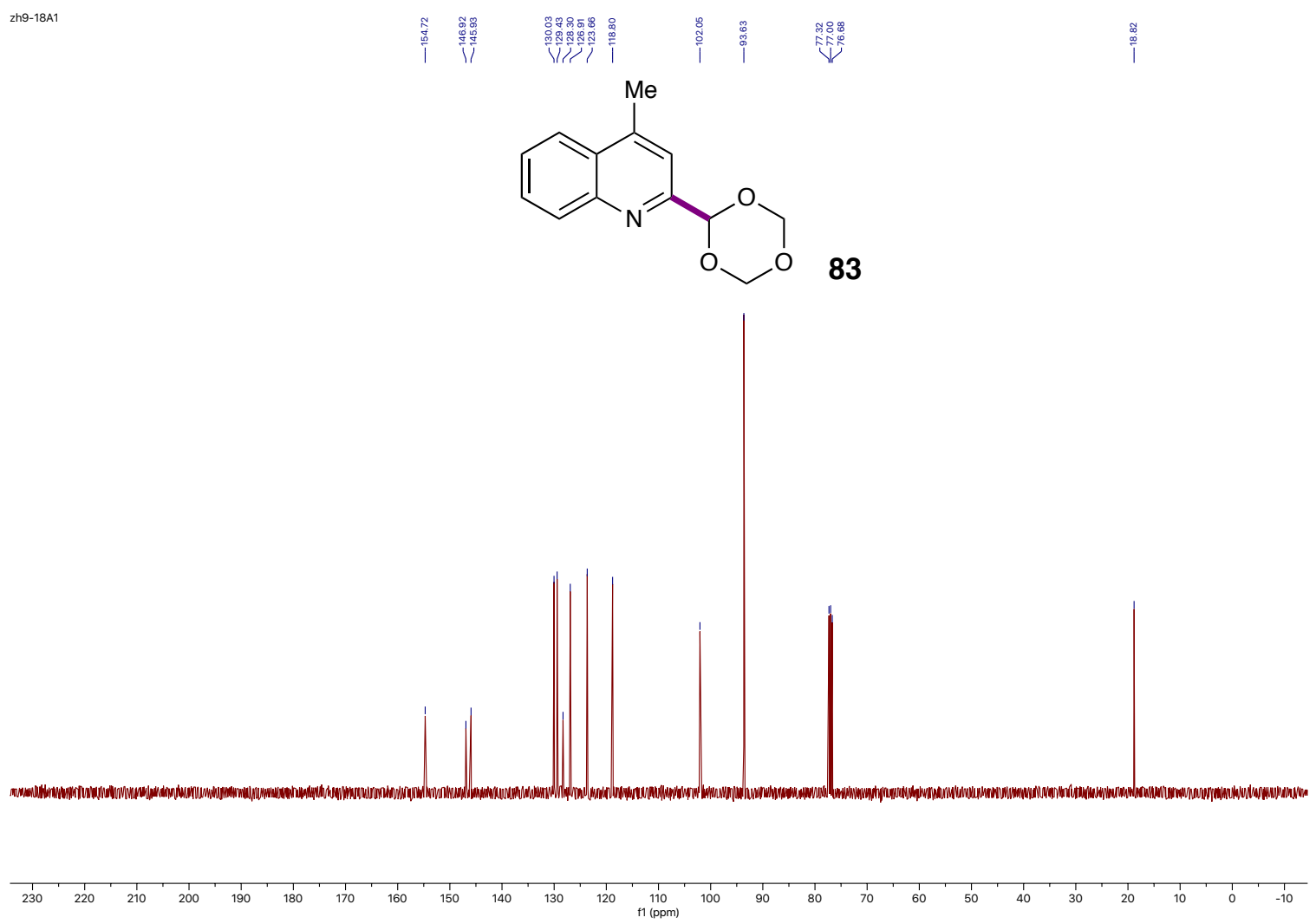
2011746-zh-9-21A-product-3.10.fid

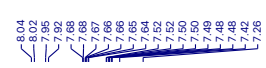

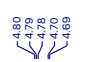

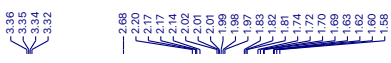

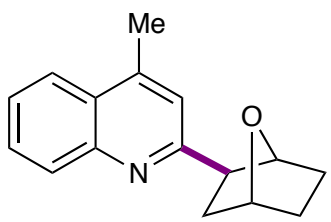

84
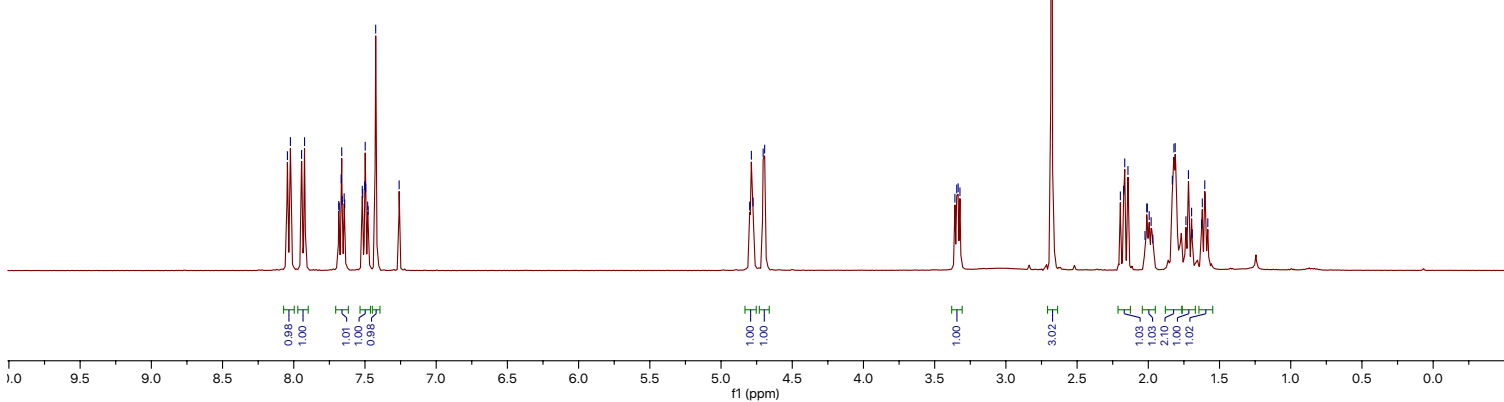

zh-9-21A
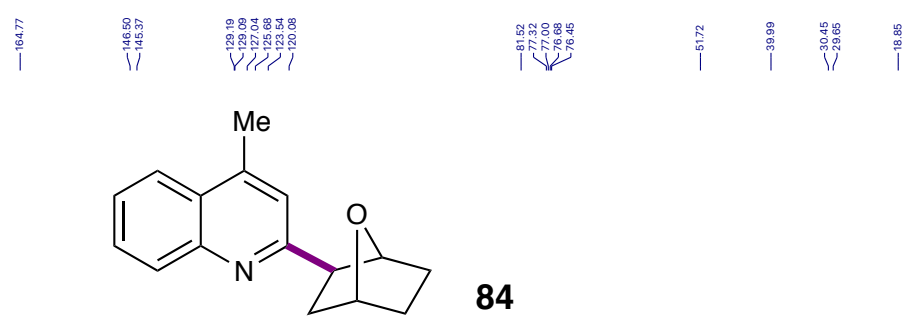

84

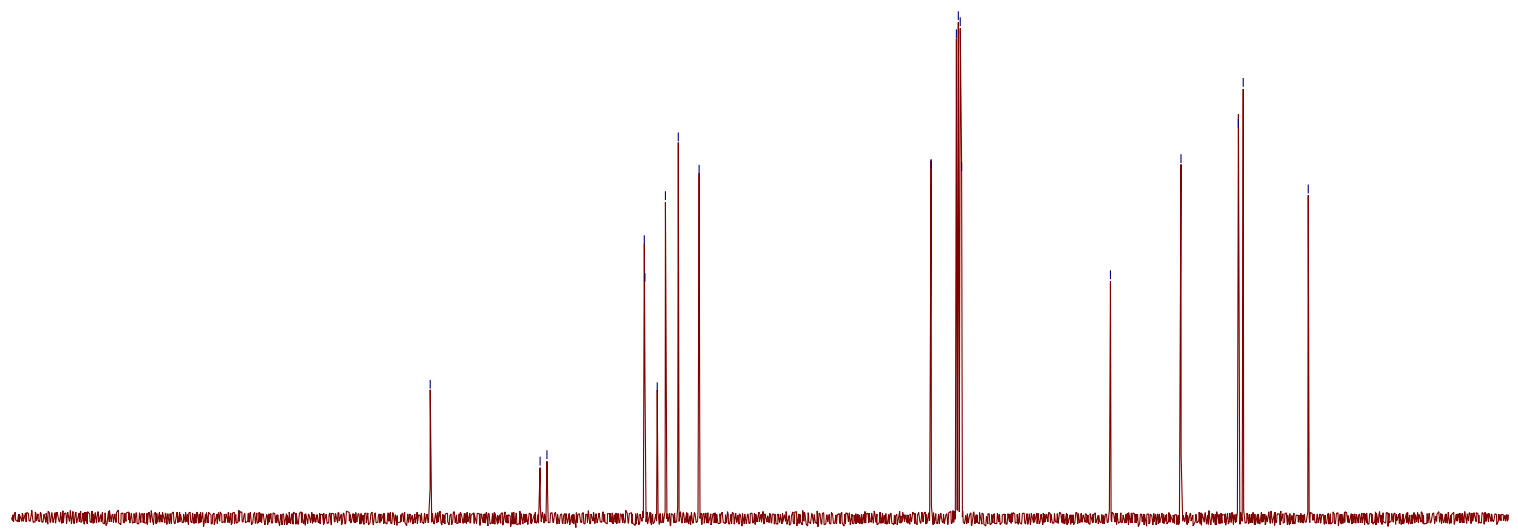

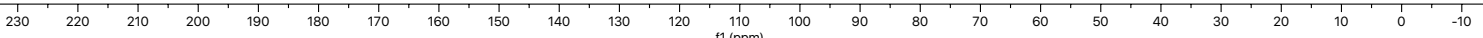



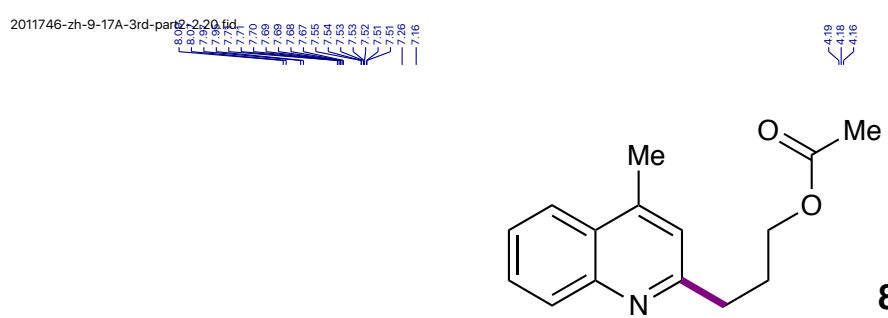

$85 a$

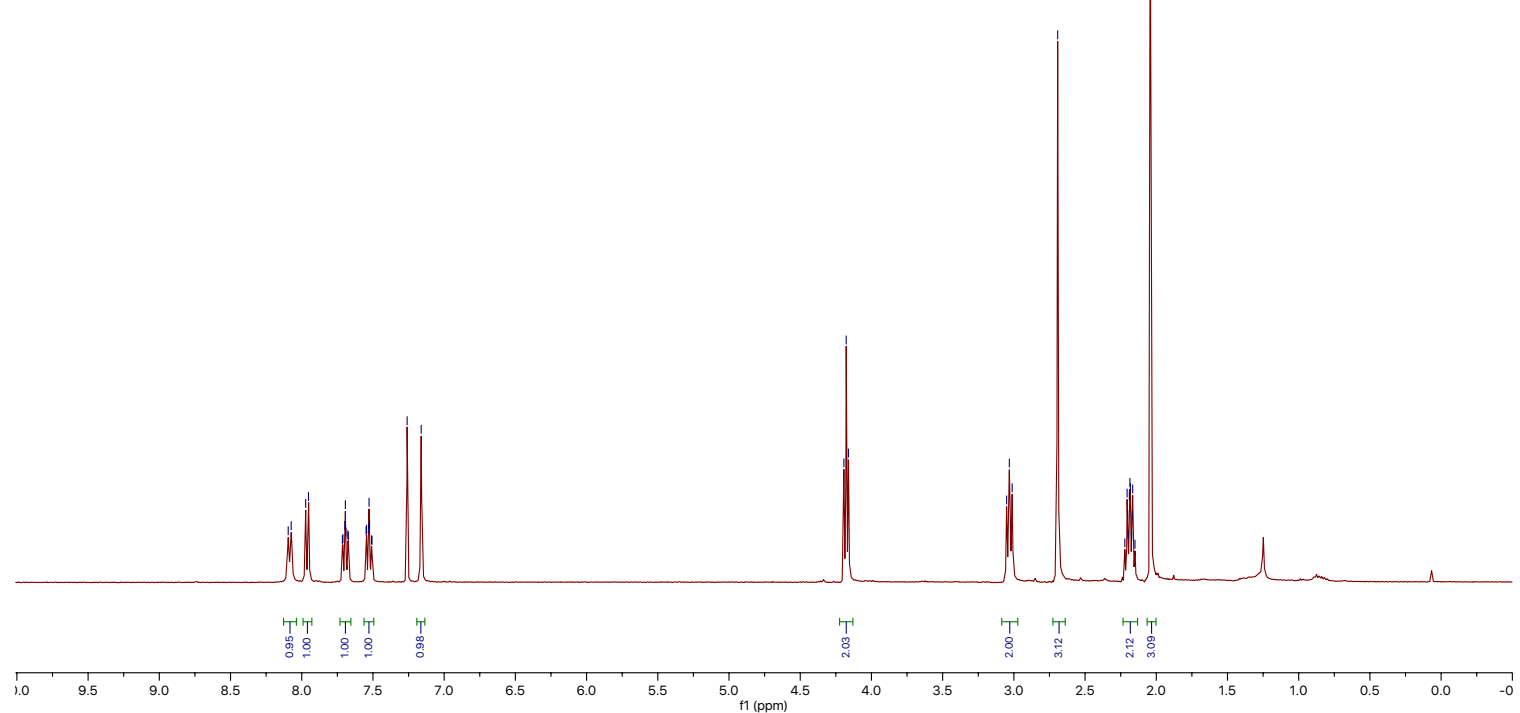

zh-9-17A-3rd
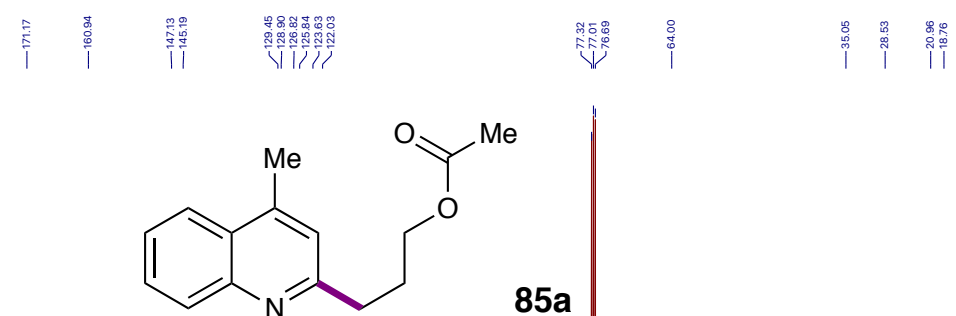

$85 a$

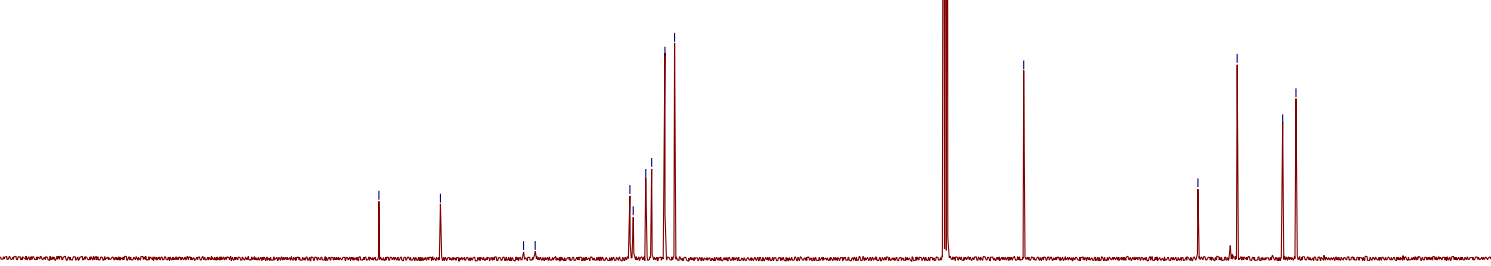

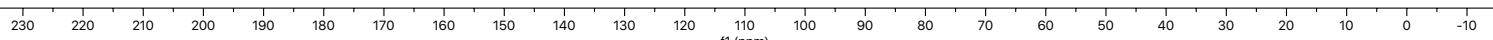




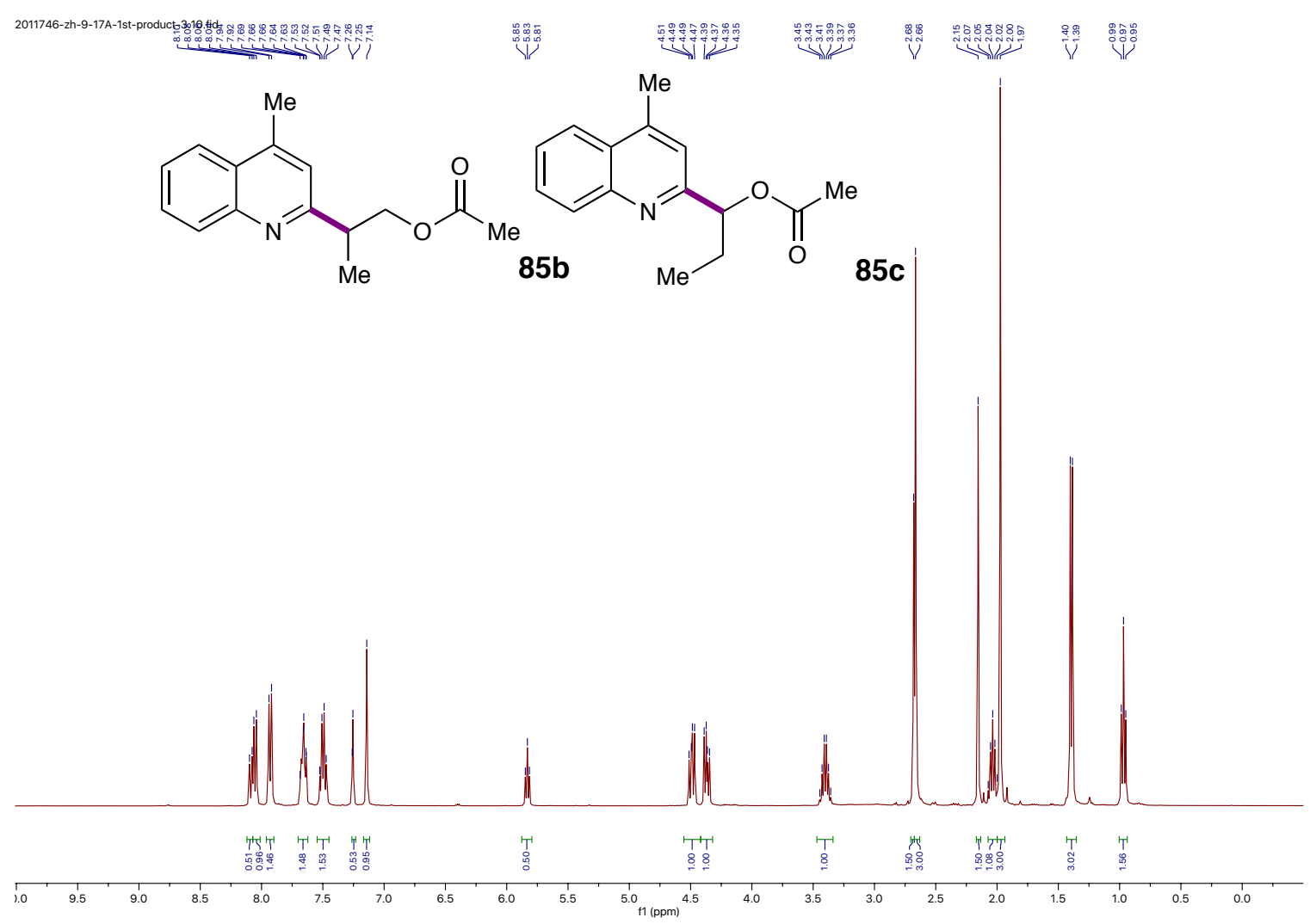

zh9-17A-1st

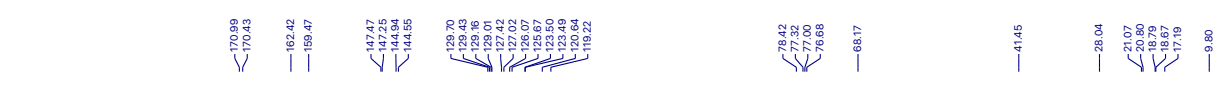<smiles>CCC(OC(C)=O)c1cc(C)c2ccccc2n1</smiles>

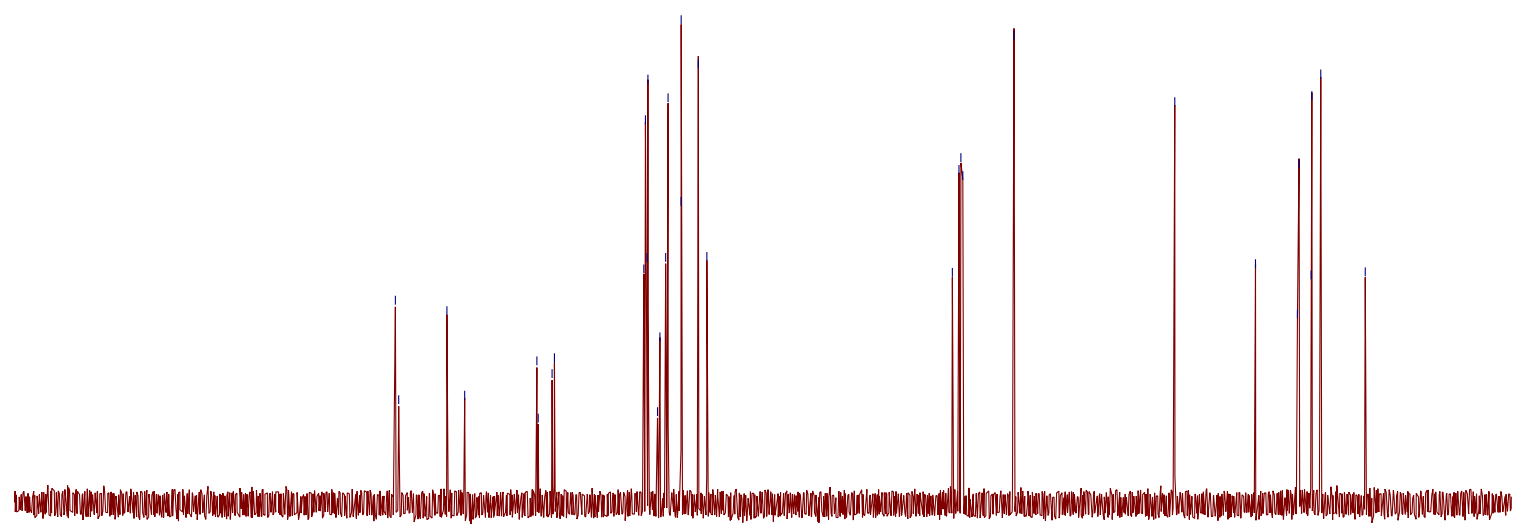

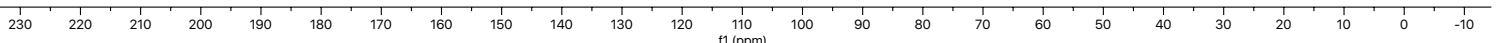




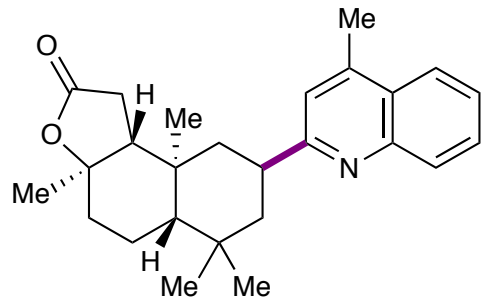

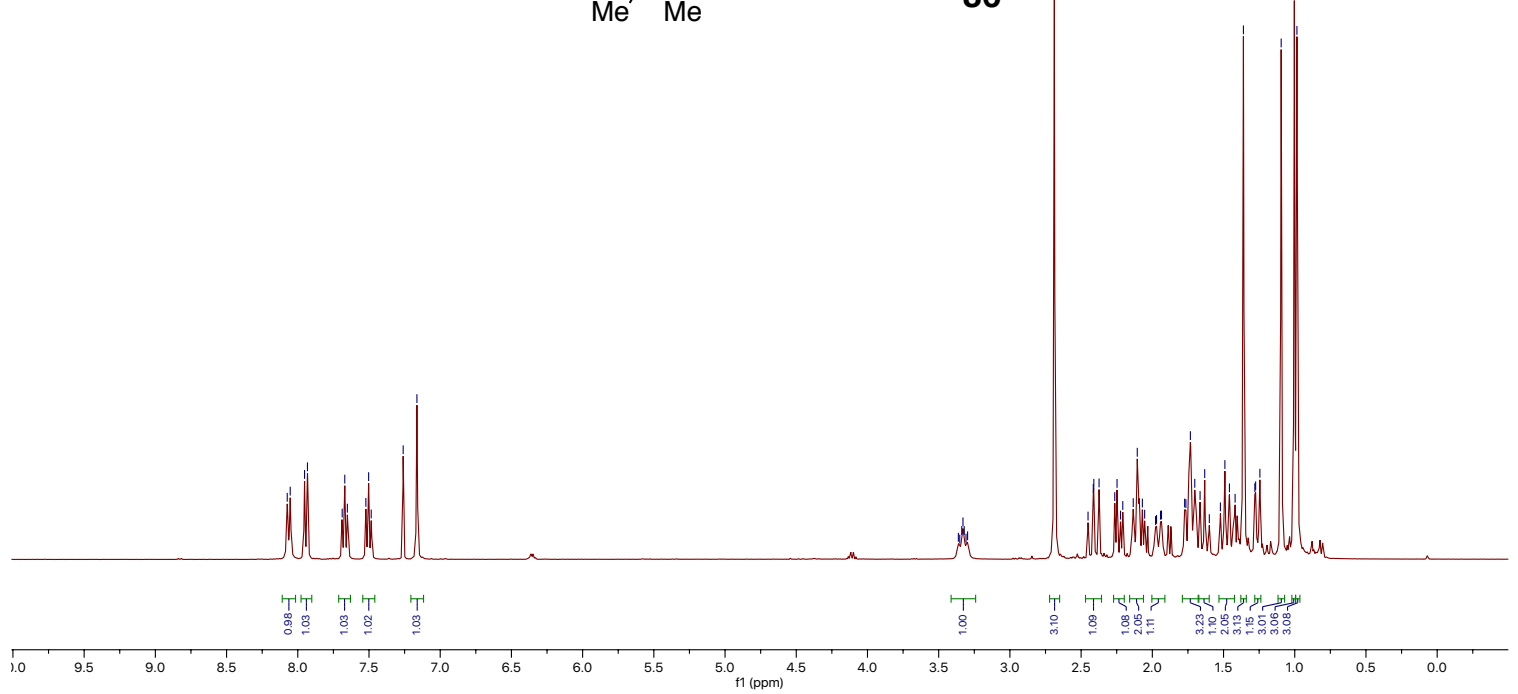

zh-8-74A1-2
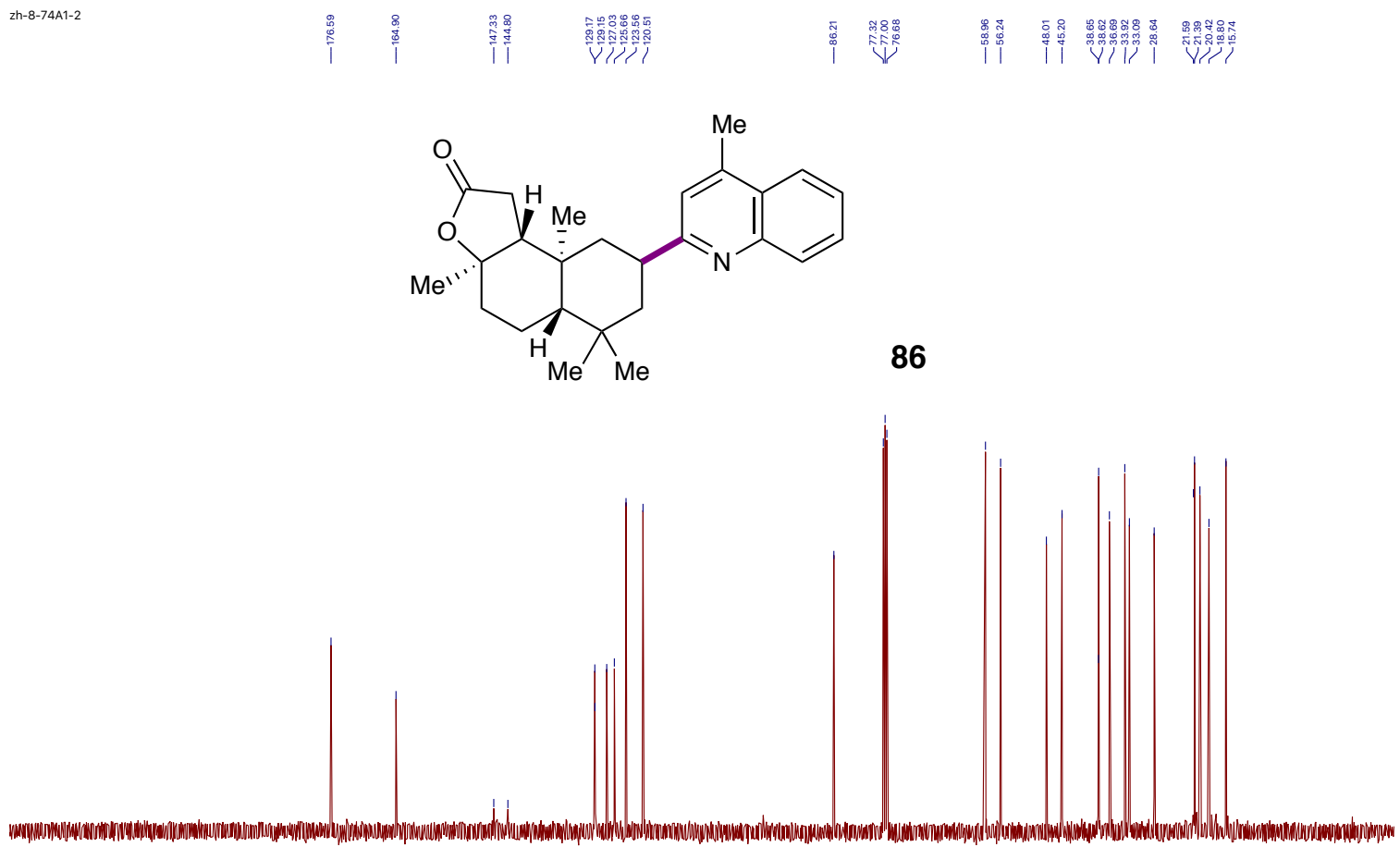

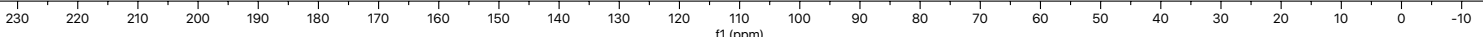

